Florida International University FIU Digital Commons

2-28-2014

\title{
Covalent Protein Adduction of Nitrogen Mustards and Related Compounds
}

Vanessa R. Thompson

Florida International University, vthompson3120@gmail.com

DOI: $10.25148 /$ etd.FI14040835

Follow this and additional works at: https://digitalcommons.fiu.edu/etd

Part of the Amino Acids, Peptides, and Proteins Commons, and the Analytical Chemistry Commons

\section{Recommended Citation}

Thompson, Vanessa R., "Covalent Protein Adduction of Nitrogen Mustards and Related Compounds" (2014). FIU Electronic Theses and Dissertations. 1152.

https://digitalcommons.fiu.edu/etd/1152

This work is brought to you for free and open access by the University Graduate School at FIU Digital Commons. It has been accepted for inclusion in FIU Electronic Theses and Dissertations by an authorized administrator of FIU Digital Commons. For more information, please contact dcc@fiu.edu. 


\title{
FLORIDA INTERNATIONAL UNIVERSITY
}

Miami, Florida

\section{COVALENT PROTEIN ADDUCTION OF NITROGEN MUSTARDS AND RELATED COMPOUNDS}

\author{
A dissertation submitted in partial fulfillment of \\ the requirements for the degree of \\ DOCTOR OF PHILOSOPHY \\ in \\ CHEMISTRY \\ by \\ Vanessa Thompson
}

2014 


\section{To: Dean Kenneth G. Furton}

College of Arts and Sciences

This dissertation, written by Vanessa Thompson, and entitled Covalent Protein Adduction of Nitrogen Mustards and Related Compounds, having been approved in respect to style and intellectual content, is referred to you for judgment.

We have read this dissertation and recommend that it be approved.

Fenfei Leng

$\begin{array}{r}\hline \text { Watson Lees } \\ \hline \text { Dietrich Lorke } \\ \hline \text { Bruce McCord } \\ \hline \text { Anthony DeCaprio, Major Professor }\end{array}$

Date of Defense: February 27, 2014

The dissertation of Vanessa Thompson is approved.

Dean Kenneth G. Furton College of Arts and Sciences

Dean Lakshmi N. Reddi University Graduate School

Florida International University, 2014 
(C) Copyright 2014 by Vanessa Thompson

All rights reserved. 


\section{DEDICATION}

I would like to dedicate this work to my parents, who supported me throughout this journey and who always encouraged me to follow my dreams, no matter what. Your love has meant the world to me, and I couldn't be more thankful. 


\section{ACKNOWLEDGMENTS}

First, I wish to acknowledge my lab mates - specifically Dr. Kevin Schneider, Dr.

Carolina Möller, and Dr. Madeleine Swortwood - for their invaluable help and encouragement throughout my doctoral studies. The three of you have taught me so much, and I cannot thank you enough for your love and support. Second, I wish to acknowledge Dr. Anthony DeCaprio for giving me the opportunity to work in a field that I am truly passionate about. In addition, I would like to acknowledge my dissertation committee - Dr. Fenfei Leng, Dr. Watson Lees, Dr.

Dietrich Lorke, and Dr. Bruce McCord - for their wisdom and advice during the progression of this project. Third, I wish to acknowledge the support of my friends and family during my graduate school career. 


\section{ABSTRACT OF THE DISSERTATION \\ COVALENT PROTEIN ADDUCTION OF NITROGEN MUSTARDS AND RELATED \\ COMPOUNDS}

by

Vanessa Thompson

Florida International University, 2014

Miami, Florida

Professor Anthony DeCaprio, Major Professor

Chemical warfare agents continue to pose a global threat despite the efforts of the international community to prohibit their use in warfare. For this reason, improvement in the detection of these compounds remains of forensic interest. Protein adducts formed by the covalent modification of an electrophilic xenobiotic and a nucleophilic amino acid may provide a biomarker of exposure that is stable and specific to compounds of interest (such as chemical warfare agents), and have the capability to extend the window of detection further than the parent compound or circulating metabolites.

This research investigated the formation of protein adducts of the nitrogen mustard chemical warfare agents mechlorethamine $(\mathrm{HN}-2)$ and tris(2-chloroethyl)amine (HN-3) to lysine and histidine residues found on the blood proteins hemoglobin and human serum albumin. Identified adducts were assessed for reproducibility and stability both in model peptide and whole protein assays. Specificity of these identified adducts was assessed using in vitro assays to metabolize common therapeutic drugs containing nitrogen mustard moieties. Results of the model peptide assays demonstrated that $\mathrm{HN}-2$ and $\mathrm{HN}-3$ were able to form stable adducts with lysine and histidine residues under physiological conditions. Results for whole protein assays identified three histidine adducts on hemoglobin, and three adducts (two lysine residues and one histidine residue) on human serum albumin that were previously unknown. These protein adducts were 
determined to be reproducible and stable at physiological conditions over a three-week analysis period. Results from the in vitro metabolic assays revealed that adducts formed by HN-2 and HN3 are specific to these agents, as metabolized therapeutic drugs (chlorambucil, cyclophosphamide, and melphalan) did not form the same adducts on lysine or histidine residues as the previously identified adducts formed by HN-2 and HN-3. Results obtained from the model peptide and full protein work were enhanced by comparing experimental data to theoretical calculations for adduct formation, providing further confirmatory data. This project was successful in identifying and characterizing biomarkers of exposure to $\mathrm{HN}-2$ and $\mathrm{HN}-3$ that are specific and stable and which have the potential to be used for the forensic determination of exposure to these dangerous agents. 


\section{TABLE OF CONTENTS}

CHAPTER

PAGE

1. INTRODUCTION 1

2. LITERATURE REVIEW 2

2.1. Chemical Warfare Agents 2

2.2. Biomarkers of Exposure 11

2.3. Protein Adduction Mechanism 16

2.4. Protein Adduction by Chemical Warfare Agents 20

2.5. Bottom-Up Proteomics 22

2.6. Nitrogen Mustard Therapeutic Agents 28

2.7. In Vitro Metabolic Assays 31

2.8. Research Objectives 33

3. METHODOLOGY 36

3.1. Instrumentation 36

3.2. Model Peptide Adduct Screening 35

3.3. Full Protein Adduction Studies 38

3.4. Whole Blood Studies 41

3.5. In Vitro Metabolism of Nitrogen Mustard Therapeutics 43

3.6. Quantum Mechanical Calculations 44

4. RESULTS AND DISCUSSION 46

4.1. Model Peptide Adduct Screening 46

4.1.1. HN-2/HN-3 Adduction to AcPAACAA 46

4.1.2. $\mathrm{HN}-2 / \mathrm{HN}-3$ Adduction to AcPAAKAA 49

4.1.3.HN-2/HN-3 Adduction to AcPAAHAA 52

4.1.4. Specificity of Indentified Adducts based on Precursor Incubations 54

4.1.5. Formation Kinetics of Model Peptide Adducts $\quad 55$

4.1.6. Stability of Model Peptide Adducts $\quad 60$

$\begin{array}{ll}\text { 4.1.7.Discussion } & 63\end{array}$

4.2. Full Protein Adduction Studies $\quad 66$

4.2.1. Direct Injection Analysis of Full Proteins $\quad 70$

4.2.2. HN-2 Adduction to Hemoglobin 72

4.2.3. HN-3 Adduction to Hemoglobin $\quad 76$

4.2.4. HN-2 Adduction to Human Serum Albumin $\quad 80$

4.2.5. HN-3 Adduction to Human Serum Albumin $\quad 85$

4.2.6. Stability of Full Protein Adducts $\quad 88$

4.2.7. Discussion 90

4.3. Whole Blood Incubation and In Vitro Protein Adduction 95

4.4. In Vitro Metabolism of Nitrogen Mustard Therapeutic Compounds 101

$\begin{array}{ll}\text { 4.4.1. Cyclophosphamide } & 102\end{array}$

$\begin{array}{ll}\text { 4.4.2. Chlorambucil } & 104\end{array}$

4.4.3. Melphalan 106

$\begin{array}{ll}\text { 4.4.4. Discussion } & 108\end{array}$

4.5. Quantum Mechanical Calculations 110

4.5.1.Electrophilicity of Nitrogen Mustards and Related Intermediates 110 
Table 1: Schedule I CWA as determined by the Chemical Weapons Convention

Table 2: Various compositional permutations of Sulfur Mustard

Table 3: Most common DNA and protein residues for electrophilic adduction

Table 4: Components of in vitro metabolic system

Table 5: Criteria for positive identification of protein adducts following HN-2 and HN-3 incubation

Table 6: Stability of identified $\mathrm{HN}-2$ adducts to $\mathrm{Hb}$ and HSA over three weeks

Table 7: Stability of identified HN-3 adducts to $\mathrm{Hb}$ and HSA over three weeks

Table 8: Calculated chemical potentials $(\mu)$ and hardness values $(\eta)$ for HN-2 and related intermediates

Table 9: Calculated chemical potentials $(\mu)$ and hardness values $(\eta)$ for HN-3 and related intermediates

Table 10: Calculated chemical potentials and hardness values for amino acids studied in this work

Table 11: Calculated chemical potential and hardness values for ionizable endogenous amino acids

Table 12: Calculated reactivity index for $\mathrm{HN}-2 \mathrm{Az} \mathrm{Cl}$ to nucleophilic amino acids studied in this work

Table 13: Calculated reactivity index for $\mathrm{HN}-2 \mathrm{Az} \mathrm{Cl}$ for ionizable amino acids

Table 14: Calculated reactivity index for $\mathrm{HN}-3 \mathrm{Az} \mathrm{Cl} \mathrm{Cl}$ to nucleophilic amino acids studied in this work

Table 15: Calculated reactivity index values for $\mathrm{HN}-3 \mathrm{Az} \mathrm{Cl} \mathrm{Cl}$ to ionizable amino acids 


\section{LIST OF FIGURES}

FIGURE

PAGE

Figure 1: Mechanism of OP toxicity $\quad 6$

Figure 2: Mechanism of OP binding to active Ser on AChE, followed by ageing process 7

Figure 3: Hydrolysis of Lewisite to CVAA; structure of British anti Lewisite 10

Figure 4: Formation of aziridinium ions for nitrogen mustard compounds $\quad 10$

Figure 5: Bottom-up versus top-down proteomics 23

Figure 6: Description of peptide MS-MS fragments 23

Figure 7: Positive mode electrospray ionization (ESI) 26

Figure 8: Various modes for QQQ analysis 27

Figure 9: Schematic of quadrupole-time-of-flight (Q-TOF) mass analyzer 28

Figure 10: Common therapeutic drugs containing nitrogen mustard moiety 30

Figure 11: Fate of drugs and xenobiotics in the body 32

Figure 12: HN-2 adduction to AcPAACAA $\quad 47$

Figure 13: HN-3 adduction to AcPAACAA 48

Figure 14: HN-2 adduction to AcPAAKAA 50

Figure 15: HN-3 adduction to AcPAAKAA

Figure 16: HN-2 adduction to AcPAAHAA

Figure 17: HN-3 adduction to AcPAAHAA 54

Figure 18: Structure of $\mathrm{HN}-2$ and $\mathrm{HN}-3$ precursors N-methyldiethanolamine (HN-2) and triethanolamine $(\mathrm{HN}-3)$

Figure 19: Relative kinetics of formation of $\mathrm{HN}-2$ and $\mathrm{HN}-3$ adducts to AcPAACAA 56

Figure 20: Relative kinetics of formation of HN-2 and HN-3 adducts to AcPAAKAA 57

Figure 21: Relative kinetics of formation of $\mathrm{HN}-2$ and $\mathrm{HN}-3$ adducts to AcPAAHAA 58 
Figure 22: Comparison of formation kinetics for HN-2 and HN-3 to AcPAACAA, AcPAAKAA and AcPAAHAA

Figure 23: Structures of identified peptide adducts $\quad 61$

Figure 24: Stability of HN-2 and HN-3 peptide adducts over three weeks 63

Figure 25: Data processing workflow utilizing BioConfirm and Protein Prospector $\quad 70$

Figure 26: MS-MS identification of $\mathrm{Hb} \alpha \mathrm{N}$-terminal adduction by $\mathrm{HN}-2$

Figure 27: MS-MS identification of $\mathrm{Hb} \beta$ N-terminal adduction by $\mathrm{HN}-2$

Figure 28: MS-MS identification of Hb His-50 $\alpha$ adduction by HN-2 74

Figure 29: MS-MS identification of Hb His-77 $\beta$ adduction by HN-2 75

Figure 30: MS-MS identification of Hb His-143 $\beta$ adduction by HN-2 76

Figure 31: MS-MS identification of $\mathrm{Hb} \beta$ N-terminal adduction by HN-3 77

Figure 32: MS-MS identification of Hb His-50 $\alpha$ adduction by HN-3 78

Figure 33: MS-MS identification of Hb His-77 $\beta$ adduction by HN-3 79

Figure 34: MS-MS identification of Hb His-143 $\beta$ adduction by HN-3 80

Figure 35: MS-MS identification of HSA N-terminal modification by HN-2 81

Figure 36: MS-MS identification of HSA Lys-233 adduction by HN-2 82

Figure 37: MS-MS identification of HSA Lys-317 adduction by HN-2 83

Figure 38: MS-MS identification of HSA His-367 adduction by HN-2 84

Figure 39: MS-MS identification of HSA N-terminal adduction by HN-3 85

Figure 40: MS-MS identification of HSA Lys-233 adduction by HN-3 86

Figure 41: MS-MS identification of HSA Lys-317 adduction by HN-3 87

Figure 42: Space-filled model of Hb, with highlighted Lys and His residues 91

Figure 43: Space-filled model of HSA, with highlighted Lys and His residues 92

Figure 44: C8 analysis of extracted $\mathrm{Hb}$ as compared to a standard 96

Figure 45: C8 analysis of extracted HSA as compared to a standard 97 
Figure 46: Comparison of the $\mathrm{Hb}$ amino acid sequences of human and rat

Figure 47: Comparison of SA amino acid sequences of human and rat

Figure 48: Structures of nitrogen mustard containing therapeutic agents for analysis

Figure 49: S9-metabolism of CP, as compared to control samples

Figure 50: HLM-metabolized CP, as compared to control samples

Figure 51: S9-metabolized of CB, as compared to control samples

Figure 52: HLM-metabolized CB, as compared to control samples 106

Figure 53: S9-metabolized MP, as compared to control samples 107

Figure 54: HLM-metabolized MP, as compared to control samples 


\section{ABBREVIATIONS AND ACRYONYMS}

2-PAM

$\mathrm{ACh}$

AChE

AcPAACAA

AcPAAHAA

AcPAAKAA

AGT

Ala

AmBic

Arg

Asp

$\mathrm{Az}$

BAL

BuChE

CB

CE

CEES

$\mathrm{CP}$

CVAA

CWA

CWC

CYP450

Cys

DNA pralidoxime; 2-pyridine aldoxime

acetylcholine

acetylcholinesterase

Acetyl-Pro-Ala-Ala-Cys-Ala-Ala

Acetyl-Pro-Ala-Ala-His-Ala-Ala

Acetyl-Pro-Ala-Ala-Lys-Ala-Ala

$\mathrm{O}^{6}$-alkylguanine DNA alkyltransferase

alanine

ammonium bicarbonate

arginine

aspartic acid

aziridinium

British Anti Lewisite; dimercaprol

butyrylcholinesterase

chlorambucil

capillary electrophoresis

2-chloroethyl ethyl sulfide

cyclophosphamide

2-chlorovinylarsonous acid

chemical warfare agent

Chemical Weapons Convention

cytochrome P-450

cysteine

deoxyribonucleic acid 


\begin{tabular}{|c|c|}
\hline ESI & electrospray ionization \\
\hline FMO & flavin-containing monooxygenases \\
\hline GC-MS & gas chromatography mass spectrometry \\
\hline Glu & glutamic acid \\
\hline GSH & glutathione \\
\hline GST & glutathione-S-transferase \\
\hline $\mathrm{H} /$ Agent $\mathrm{H}$ & Levenstein Mustard \\
\hline $\mathrm{Hb}$ & hemoglobin \\
\hline HD & sulfur mustard; bis-(2-chloroethyl)sulfide \\
\hline His & histidine \\
\hline HL & Sulfur mustard-Lewisite mixture \\
\hline HLM & human liver microsomes \\
\hline $\mathrm{HN}$ & nitrogen mustard (generic) \\
\hline $\mathrm{HN}-2$ & mechlorethamine \\
\hline $\mathrm{HN}-3$ & tris(2-chloroethyl)amine \\
\hline HOMO & highest unoccupied molecular orbital \\
\hline HPLC & high performance liquid chromatography \\
\hline HQ & mix of bis-(2-chloroethyl)sulfide and 1,2-bis(2-chloroethylthio)ethane \\
\hline HSA & human serum albumin \\
\hline HSAB & Hard and Soft Acid-Base \\
\hline $\mathrm{LC}$ & liquid chromatography \\
\hline LC-MS & liquid chromatography mass spectrometry \\
\hline LC-MS-MS & liquid chromatography tandem mass spectrometry \\
\hline LUMO & lowest unoccupied molecular orbital \\
\hline Lys & lysine \\
\hline
\end{tabular}




\begin{tabular}{|c|c|}
\hline MALDI & Matrix Assisted Laser Desorption Ionization \\
\hline MAO & monoamine oxidase \\
\hline MP & melphalan \\
\hline MRM & multiple reaction monitoring \\
\hline MS & mass spectrometry \\
\hline MS-MS & tandem mass spectrometry \\
\hline $\mathrm{OP}$ & organophosphate \\
\hline OPCW & Organization for the Prohibition of Chemical Weapons \\
\hline Phe & phenylalanine \\
\hline Pro & proline \\
\hline Q & sesqui mustard \\
\hline QQQ & triple quadrupole \\
\hline Q-TOF & quadrupole-time-of-flight \\
\hline RT & retention time \\
\hline Ser & serine \\
\hline SIM & single ion monitoring \\
\hline SPB & sodium phosphate buffer \\
\hline SPE & solid phase extraction \\
\hline TCEP & tris(2-carboxyethyl)phosphine \\
\hline TFA & trifluoroacetic acid \\
\hline Thr & threonine \\
\hline TOF & time-of-flight \\
\hline Tyr & tyrosine \\
\hline Val & valine \\
\hline WWI & World War I \\
\hline
\end{tabular}




\section{INTRODUCTION}

The history of chemical and biological warfare agents can be traced back thousands of years. Since World War I, the advancement of chemical weapons has been quite drastic, and despite the efforts of the international community to ban the use of these chemicals during warfare, they are still utilized today. For this reason, it is important for the forensic community to develop methods for their detection not only in environmental samples, but in human specimens as well. Two challenges to the detection and identification of these compounds in biological materials involve the limited time in which the parent compounds are present in the blood and the low specificity of circulating metabolites. As such, a different approach to their detection is necessary. Protein adduction by reactive xenobiotics, including chemical weapons, may provide a method for exposure determination that is not only specific to the compound of interest, but also provides a longer lasting exposure biomarker as compared to the parent compound or metabolites.

The current research was performed to identify and characterize potential protein adduct biomarkers of exposure to the Schedule I chemical warfare agent nitrogen mustards mechlorethamine (HN-2) and tris(2-chloroethyl)amine (HN-3) to lysine and histidine residues on the blood proteins hemoglobin and serum albumin using liquid chromatography tandem mass spectrometry. Identified adducts were assessed for their specificity as HN-2 and HN-3 exposure markers as compared to other compounds containing nitrogen mustard moieties, including ethanolamine precursor compounds and alkylating cancer drugs. Adducts were also assessed for their stability at physiological conditions $\left(37^{\circ} \mathrm{C}, \mathrm{pH} 7.4\right)$, which would lend confidence to the use of identified adducts as potential biomarkers of exposure for nitrogen mustard chemical weapons. This research has not only filled an important gap in nitrogen mustard protein adduction research, but has also provided evidence for additional avenues of adduction exploration for these forensically important compounds. 


\section{LITERATURE REVIEW}

\subsection{Chemical Warfare Agents}

Chemical warfare agents (CWA) are defined as chemicals that are used to incapacitate, injure, or kill an individual or group of individuals. ${ }^{1}$ The use of chemical weapons in some form or fashion can be traced back to ancient times, when armies would use toxic or incapacitating chemicals (such as sulfur) along with biological agents (such as poisonous plants or animals) to kill or make enemies sick. ${ }^{2}$ The first large-scale use of chemical weapons during wartime occurred in World War I (WWI), where chlorine gas was released by German forces at Ypres. ${ }^{2}$ This, along with other instances in WWI in which other chemical weapons were utilized (including phosgene, sulfur mustard, and cyanide), marked a time of importance for chemical weapons research. In the ensuing years, several advances were made in the CWA field, including the use and development of sulfur mustard (HD) in 1917 and the organophosphate nerve agents in the 1930 s. $^{2}$

Parallel with the development and "improvement" of these chemical weapons were attempts by the international community to prohibit the use of both chemical and biological agents during wartime because of their potentially devastating effects. The most recent attempt at control of these compounds occurred when the Organization for the Prohibition of Chemical Weapons (OPCW) was formed. The committee drafted the Chemical Weapons Convention (CWC), which was signed by several countries throughout the world (including the United States) in 1993. The CWC detailed the prohibition of the use and stockpiling of chemical weapons, in addition to providing a scheduling process for these compounds for those countries that ratified the treaty. To date, 189 countries have signed the treaty and ratified it into law. Two states (Israel and Myanmar) have signed the treaty, but have yet to implement its mandate. Four states (Angola, Egypt, North Korea, and South Sudan) have not signed the treaty. ${ }^{3}$ Despite these measures, however, chemical weapons are still a threat, with recent use by both radical cults and 
militant governments. For example, in 1995 the Japanese cult group Aum Shinrikyo was responsible for the release of sarin, an organophosphate nerve agent, in the city of Matsumoto and on a Tokyo subway in 1994 and 1995, respectively. ${ }^{4}$ Most recently, the Assad regime in Syria was accused of releasing sarin on rebel forces in August of 2013. ${ }^{5}$ As a result of these attacks and to avoid backlash from the international community, Syria agreed to destroy their stockpiles and sign the CWC as of September 2013. These recent attacks serve as somber reminders to the international community that, despite the regulations and laws that have been in place for over twenty years, these chemical weapons still pose a real threat to humanity.

Table 1 demonstrates the main classes and structures of the Schedule I CWA as determined by the CWC. ${ }^{1}$ In addition to these compounds, many of their precursors are also considered Schedule I CWA. In order for these compounds to be considered Schedule I, they must, among other considerations, have been specifically developed, produced, or stockpiled for use as chemical weapons. In addition, these compounds must have "little or no use for purposes not prohibited under this Convention" - in other words, they cannot be used (or have very few uses) for anything else except for chemical warfare. 


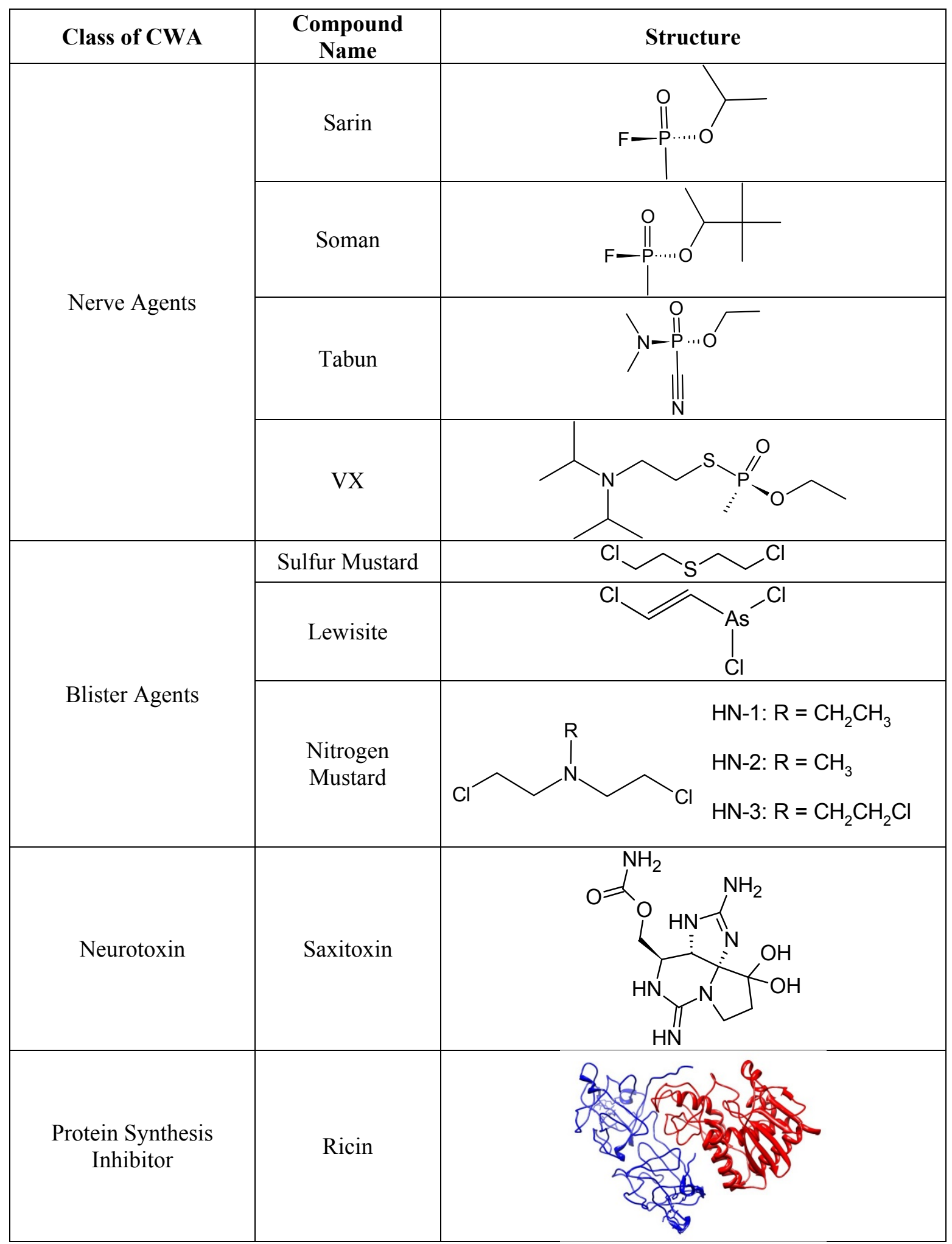

Table 1: Schedule I CWA as determined by the Chemical Weapons Convention. 
Of these CWAs, some of the most lethal agents are the organophosphate (OP) nerve agents, of which sarin is a member. Organophosphate nerve agents are related to phosphorouscontaining pesticide compounds, and their primary mode of action is to inhibit acetylcholinesterase $(\mathrm{AChE})$ and butyrylcholinesterase $(\mathrm{BuChE})$ in the body. These two enzymes are primarily responsible for the breakdown of the neurotransmitter acetylcholine (ACh) in neuronal synapses. Acetylcholine serves a variety of functions in the nervous system, including its role in the peripheral nervous system activating neuromuscular junctions via interaction with a post-synaptic binding site.

In normal biological functions, ACh is released by a pre-synaptic neuron and, when properly linked into the ACh receptor on the post-synaptic neuron, a signal for the contraction of muscles in the body is produced (Figure 1). ${ }^{6}$ Acetylcholinesterase and BuChE are responsible for the breakdown of ACh in the synapse so that it does not overstimulate the post-synaptic neuron. However, when nerve agents are present, these enzymes are blocked, due to nerve agent binding to the active serine (Ser) site on the enzyme. Consequently, $\mathrm{ACh}$ is consistently present in the synapse without means of breaking down; therefore, ACh overstimulates the post-synaptic neuron, causing continuous and uncontrollable muscular contractions. ${ }^{7}$ Eventually, an individual exposed to nerve agents without any treatment will die via asphyxiation and other physiological effects. 

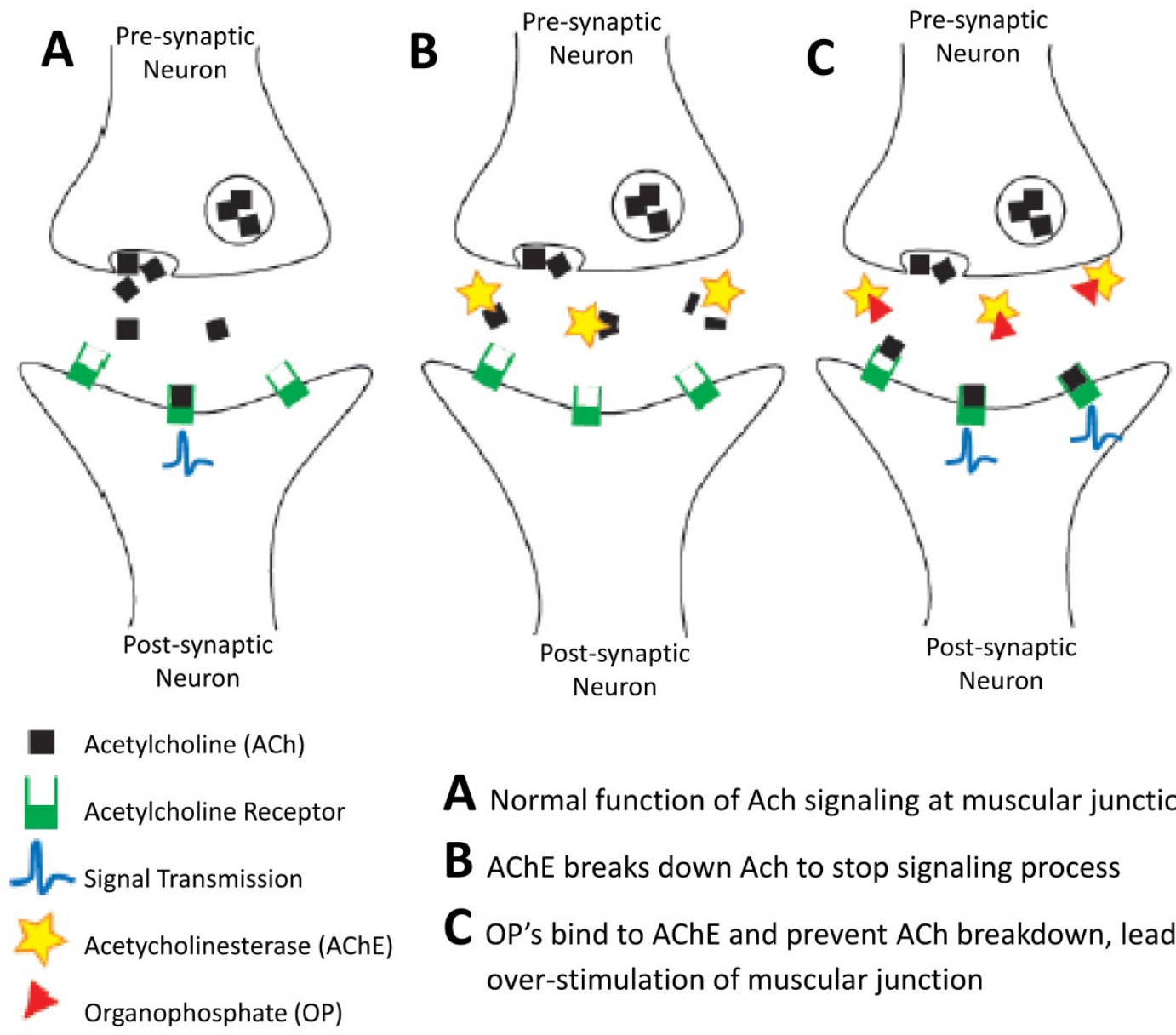
A Normal function of Ach signaling at muscular junction
B AChE breaks down Ach to stop signaling process
C OP's bind to AChE and prevent ACh breakdown, leading to over-stimulation of muscular junction

Figure 1: Mechanism of OP toxicity.

If caught early enough, nerve agent exposure can be treated with anticholinergic drugs or compounds such as pralidoxime (2-PAM) to reactivate the blocked AChE. Compounds such as 2PAM work by dephosphorylating the active Ser on AChE; however, there is a point at which 2PAM is no longer effective due to a process called "ageing" (Figure 2). Ageing occurs when the OR group (or the phosphoramide bond in the case of $\operatorname{tabun}^{8}$ ) is hydrolyzed, thus creating a stronger bond between the hydrolyzed nerve agent and the serine binding site. Once this process occurs, compounds such as 2-PAM cannot break the bond and other treatment methods (such as the administration of anticholinergic drugs) must be utilized. ${ }^{9}$ 


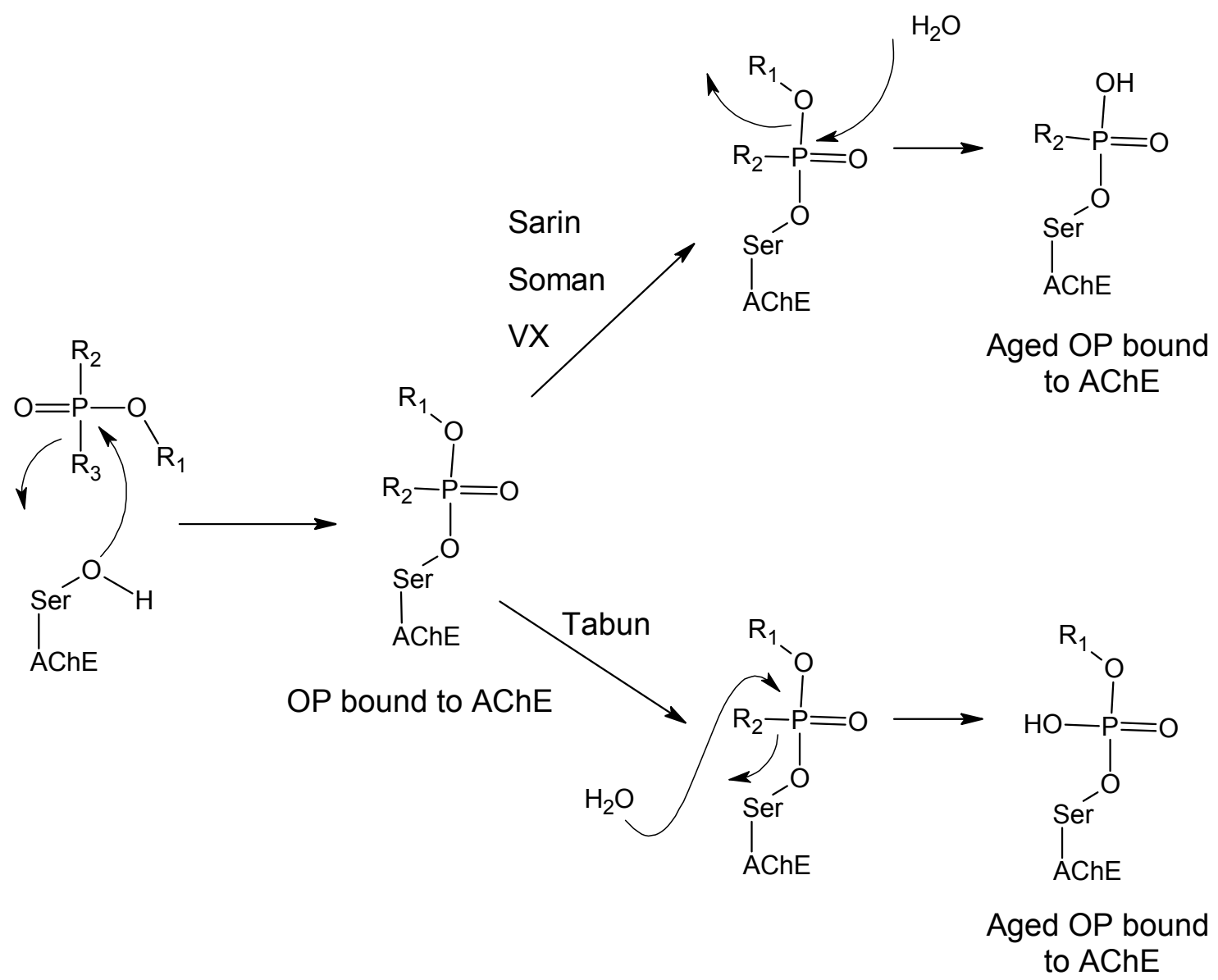

Figure 2: Mechanism of OP binding to active Ser on $\mathrm{AChE}$, followed by ageing process.

Sarin: $\mathrm{R}_{1}=\mathrm{CH}\left(\mathrm{CH}_{3}\right)_{2} ; \mathrm{R}_{2}=\mathrm{CH}_{3} ; \mathrm{R}_{3}=\mathrm{F}$

Soman: $\mathbf{R}_{1}=\mathrm{CH}\left(\mathrm{CH}_{3}\right)(t$-butyl $) ; \mathrm{R}_{2}=\mathrm{CH}_{3} \mathbf{R}_{3}=\mathrm{F}$

$V X: \mathbf{R}_{1}=\mathrm{CH}_{2} \mathrm{CH}_{3} ; \mathrm{R}_{2}=\mathrm{CH}_{3} ; \mathrm{R}_{3}=\mathrm{SCH}_{2} \mathrm{CH}_{2} \mathrm{~N}\left[\mathrm{CH}\left(\mathrm{CH}_{3}\right)_{2}\right]_{2}$

Tabun: $\mathrm{R}_{1}=\mathrm{CH}_{2} \mathrm{CH}_{3} ; \mathrm{R}_{2}=\mathrm{N}\left(\mathrm{CH}_{3}\right)_{2} ; \mathrm{R}_{3}=\mathrm{CN}$

Analytical methods for OP nerve agents are quite varied and depend on the matrix tested.

In aqueous, atmospheric, or even soil samples, hydrolysis products of nerve agents are most

stable and therefore most commonly found in these types of matrices. These hydrolysis products

tend to be polar, non-volatile compounds and thus easiest analyzed via liquid-chromatography-

mass spectrometry (LC-MS $)^{10}$ or even capillary electrophoresis $(\mathrm{CE})^{11}$. However, with

derivatization, gas chromatography-mass spectrometry (GC-MS) analysis is also possible, ${ }^{12,13}$ and

is arguably the most common method of analysis. ${ }^{14}$ In biological matrices, metabolites of these 
nerve agents may be extracted from plasma and detected directly; ${ }^{15}$ however, these techniques may suffer in terms of sensitivity, as there may not be as much free nerve agent or circulating metabolites as there are bound to $\mathrm{AChE}$ or BuChE. For this reason, biological detection of nerve agents most often occurs via a process called fluoride reactivation, in which the extracted plasma is inundated with a high concentration of fluoride ions, which can break the covalent phosphoserine $\mathrm{P}-\mathrm{O}$ bond, producing a fluorinated compound that can be detected quantitatively via GC-MS. ${ }^{16-19}$ While this method can be highly sensitive, its major drawback is if the organophosphate has aged onto the serine residue, in which case it cannot be reactivated by this method. However, it is possible to analyze the nerve agent still attached to plasma BuChE via its isolation from plasma and subsequent digestion with enzymes such as chymotrypsin ${ }^{20}$, pepsin $^{21}$, or other digestion enzymes.

Vesicants, better known as blister agents, are a second class of CWA. Blister agents are chemicals that can cause tissue blistering and ocular and respiratory damage. Unlike the nerve agents discussed previously, the action of these bister agents appears to be much more nonspecific in terms of a target enzyme or organ; however, the observed "blistering" effect appears to be a result of a separation of the epidermis from the basement membrane in the skin. ${ }^{22}$ In addition to these blistering effects, vesicants such as $\mathrm{HD}$ and the family of nitrogen mustards (HN) form highly electrophilic intermediates (sulfonium ion with $\mathrm{HD}$; aziridinium ion with $\mathrm{HN}$ ) in aqueous solutions which can bind to DNA and other macromolecules, producing a multitude of different effects including apoptotic DNA fragmentation and protein cross-linking. ${ }^{23-27}$

Of the Schedule I blister agents, HD is by far the most notorious. HD was first used by the Germans in WWI (1917), and was the main contributor of gas-related casualties during the war. ${ }^{7}$ Commonly referred to as "mustard gas" because of a potential odor imparted by synthetic impurities, HD is in fact not as gas at all, but an oily liquid that can be aerosolized for use as a chemical weapon. As a result of pure HD's low water solubility, it can be highly persistent in the 
environment, causing a problem not only for those immediately exposed, but for individuals who may pass by a contaminated area even days after. ${ }^{22}$ There are several different permutations of chemical mixtures that are classified as "sulfur mustards," which can be seen in Table $2 ;^{22}$ the designation "HD" typically refers to vacuum distilled bis(2-chloroethyl)sulfide, as seen in Table 1.

\begin{tabular}{|c|c|c|}
\hline Sulfur Mustard Type & Symbol & $\begin{array}{l}\text { Description } \\
\end{array}$ \\
\hline Distilled Mustard & HD & $\begin{array}{l}\text { bis(2-chloroethyl)sulfide (approx. 97\%) } \\
\text { achieved via vacuum distillation }\end{array}$ \\
\hline Levenstein Mustard & $\begin{array}{c}\mathrm{H} \text {, or Agent } \\
\mathrm{H}\end{array}$ & $\begin{array}{c}\text { mixture of HD (70\%) and other sulfur impurities } \\
(30 \%)\end{array}$ \\
\hline \multicolumn{2}{|l|}{ Agent HT } & $\begin{array}{l}\text { mixture of HD }(\sim 60 \%) \text { and bis }(2- \\
\text { chloroethylthioethyl)ether }(\sim 40 \%)\end{array}$ \\
\hline $\begin{array}{l}\text { Sulfur Mustard-Lewisite } \\
\text { Mixture }\end{array}$ & HL & ratios vary; developed for cold-weather use \\
\hline Sesqui Mustard & Q & 1,2-bis(2-chloroethylthio)ethane \\
\hline \multicolumn{2}{|l|}{ HQ } & mixture of $\mathrm{HD}$ and $\mathrm{Q}$ \\
\hline Half-mustard & CEES & 2-chloroethyl ethyl sulfide \\
\hline
\end{tabular}

Table 2: Various compositional permutations of "Sulfur Mustard."

A second type of Schedule I blister agent is Lewisite, an arsenic-containing vesicant.

While Lewisite was developed in the hopes of creating an improved blister agent over HD, its rapid hydrolysis to 2-chlorovinylarsonous acid (CVAA, as seen in Figure 3) in aqueous environments renders it less effective than previously hoped. Despite this shortcoming, Lewisite has been stockpiled as a mixture with HD for weapon use. ${ }^{22}$ Of all the blister agents, Lewisite is the only one with an antidote - dimercaprol, better known as British anti Lewisite (BAL) - which shows a high affinity for CVAA both in free plasma and bound to proteins. ${ }^{28}$ 


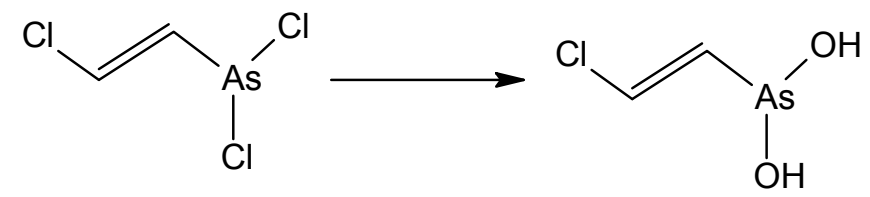

(1)

(2)

(3)

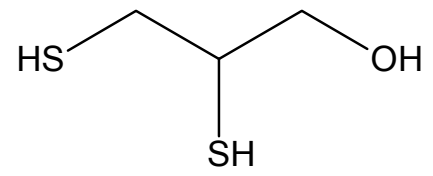

Figure 3: Hydrolysis of Lewisite (1) to CVAA (2); structure of British anti Lewisite (BAL). (3)

The third class of blister agents, and the focus of this work, is a group of compounds collectively known as nitrogen mustards (HN). Three different HN compounds are currently listed as Schedule I CWA, and can be seen in Table 1. These compounds are structurally and chemically similar to HD; therefore, their properties as CWA are similar to that of HD. The formation of the positively charged aziridinium ions (Figure 4) is largely responsible for the toxicity of HN through DNA and protein binding. ${ }^{29}$

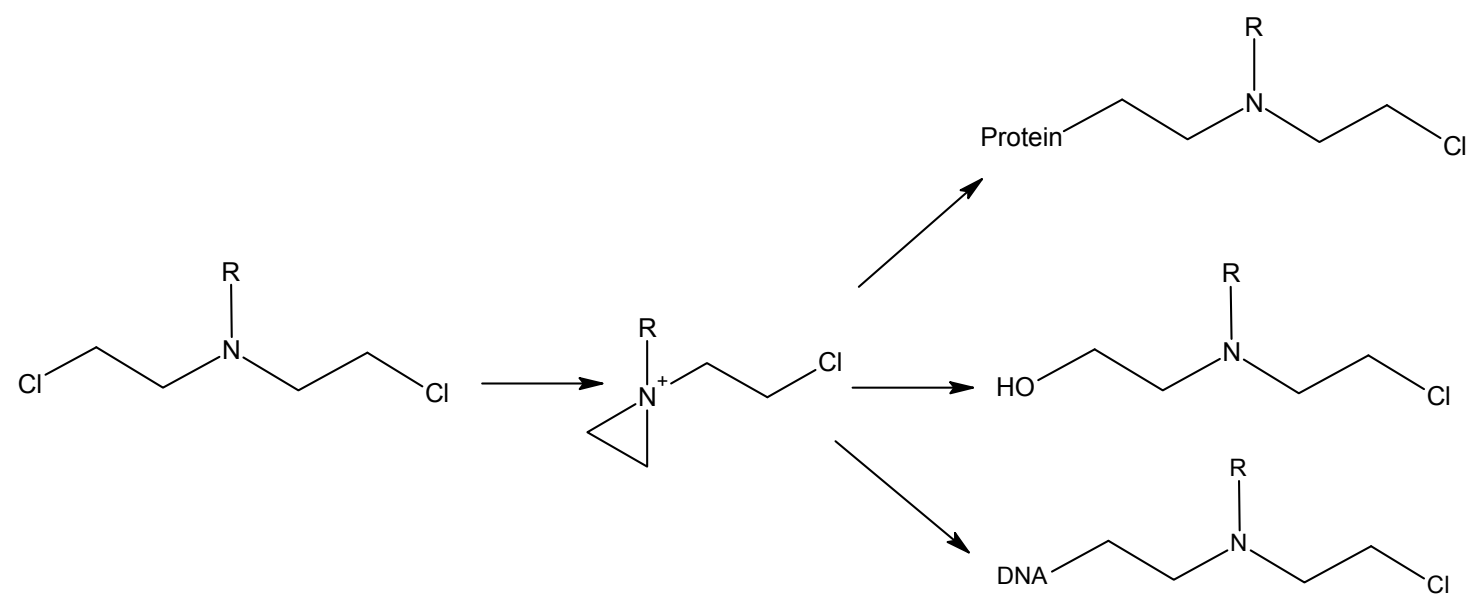

Figure 4: Formation of electrophilic aziridinium ions for nitrogen mustard compounds. The identity of the R-group defines the types of CWA (See Table 1 for structures).

Unlike the OP nerve agents, the molecular mechanism for blister agent toxicity is still largely undetermined, ${ }^{30}$ although several hypotheses exist that attempt to explain their deleterious effects. The consensus of the literature on HN compounds is that DNA and protein binding via 
the aziridinium ion is a primary component of toxicity. However, other potential mechanisms of toxicity of HN compounds have also been suggested, involving the formation of free radicals, ${ }^{23}$ oxidative damage to the lungs, ${ }^{31,32}$ the formation of reactive oxygen and reactive nitrogen species, ${ }^{33}$ and activation of inflammatory pathways, ${ }^{34}$ specifically in skin. ${ }^{35}$

As with the nerve agents, there are a variety of methods by which blister agents can be detected. When assessing environmental samples, the detection of the hydrolysis products of these compounds - CVAA for Lewisite, and the replacement of chlorine with hydroxyl groups in $\mathrm{HD}$ and the $\mathrm{HN}$ family - provides more sensitive detection of these compounds over the parent compounds. ${ }^{28,36-38}$ Most commonly, these polar compounds are detected via LC-MS or GC-MS following derivatization. ${ }^{39,40}$ The analysis of biological samples offers more variety in terms of detection. In general, HD, the HN compounds, and Lewisite have short plasma half-lives; ${ }^{41-43}$ therefore, hydrolyzed free agents in either plasma or urine tend to be the target compounds of interest. In addition, $\beta$-lyase and glutathione (GSH) conjugates have also been reported with HD exposure. ${ }^{39}$ As glutathione conjugation is a major pathway for detoxification of electrophiles in the body, ${ }^{44}$ it would also be expected that glutathione conjugates would be observed for $\mathrm{HN}$ and CVAA. $^{45,46}$ In theory, protein adducts may also be utilized for blister agent detection, once adduction sites have been properly characterized. Detection methods for such adducts involve isolation of a relevant macromolecule, digestion via one or more proteases, and peptide analysis via LC-MS. ${ }^{47}$ Protein adduction will be covered in greater depth in Section 2.4, and protein adduct analysis will be covered in Section 2.5.

\subsection{Biomarkers of Exposure}

A biomarker of exposure is defined as a biochemical alteration of some kind found in the body that can be used to determine whether an individual has been "exposed" to a xenobiotic of interest. The exposure may be intentional or unintentional - for example, an individual may take a pain reliever for a headache, representing an intentional exposure, or a worker may be exposed 
to toxic vapors as a result of industrial byproducts, which would be a result of an unintentional exposure. Regardless of intent, these exposures are represented through the presence of certain markers in the body, which, once identified, can be detected through analytical and bioanalytical methods. Exposure biomarkers are highly varied both in terms of type and specificity, ${ }^{48}$ and can include something as simple as detection of the parent compound to a more complex marker such as the covalent adduction of the compound to a macromolecule. These biomarkers are important, as ambient or environmental levels of a compound do not necessarily correlate to an individual's exposure. In contrast, biomarkers have the advantage of providing a direct connection between the compound and the individual. ${ }^{49}$

The simplest form of a biomarker is monitoring the xenobiotic of interest or its metabolites directly in a biological specimen. These types of biomarkers are most commonly utilized in forensic toxicology and the medical community in terms of drug monitoring in illicit or clinical cases, respectively. In order to use these types of biomarkers for detection, it is important to know what permutation of the compound of interest is present in the tested biological fluid. For example, a parent compound may be seen in an individual's serum; however, if urine is the matrix being analyzed, it is more likely to contain metabolites of the compound. These considerations and an understanding of the biotransformation of these compounds in the body are important for accurate determination of exposure. ${ }^{50}$

A second type of biomarker of exposure includes adducts formed on macromolecules such as DNA or protein, formed when an electrophilic xenobiotic of interest (or a reactive intermediate) forms a covalent bond with a nucleophilic residue on the macromolecule. Table 3 lists the most commonly adducted nucleophilic sites of adduction for DNA and protein. ${ }^{49}$ 


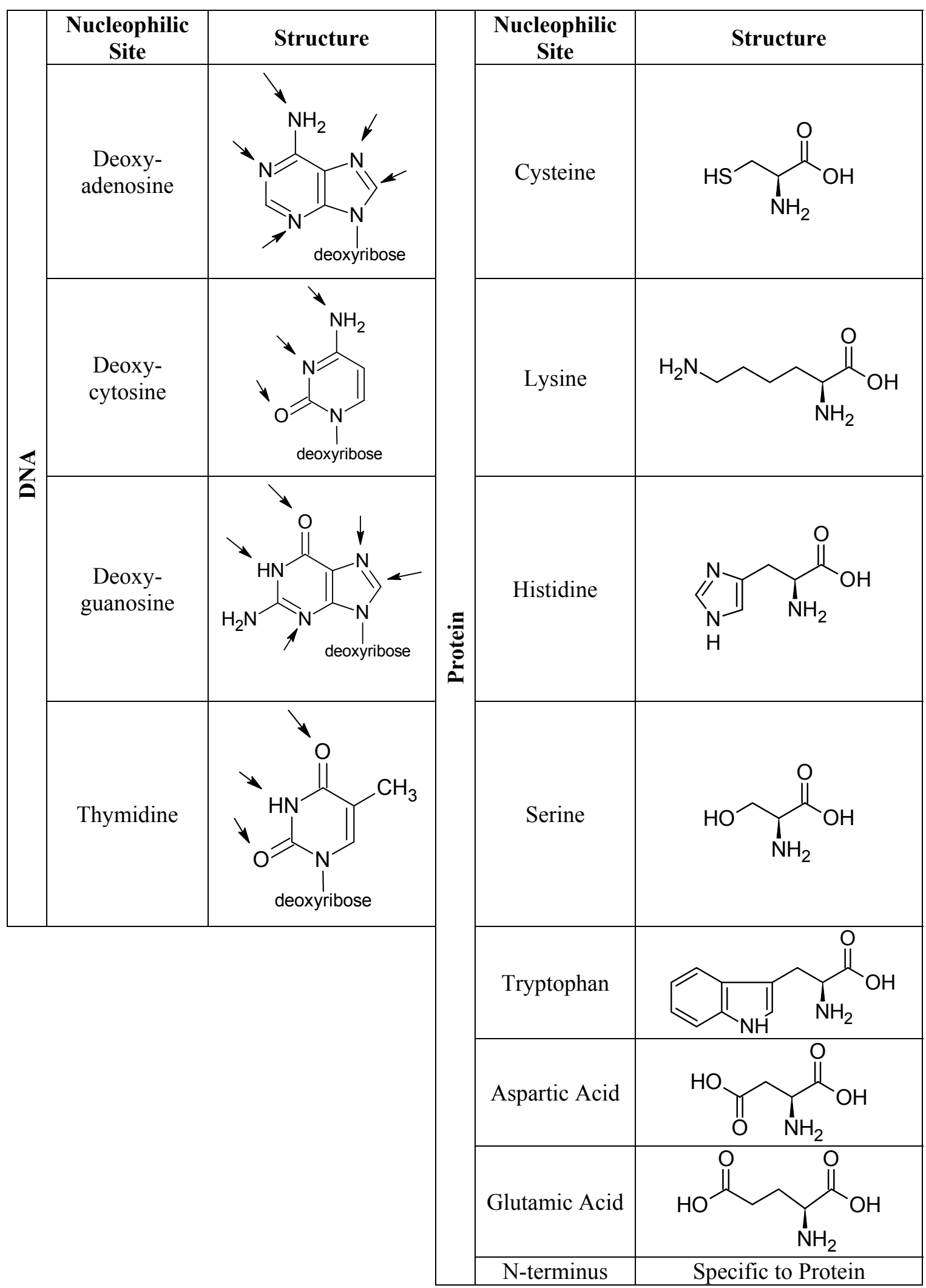

Table 3: Most common DNA and protein residues for electrophilic adduction. Arrows point to the potential reactive sites on DNA residues. 
The inherent nucleophilicity of these bases or amino acids plays an important role in whether a DNA or protein adduct will form; however, adduction cannot be predicted with this property alone. Other important factors that influence xenobiotic adduction include the relative electron density of both the nucleophile and the electrophile - in other words, the relative "hardness" or "softness" of these reactive species. ${ }^{51}$ The more compatible the nucleophile and electrophile are along this scale, the more likely a stable covalent bond will form. Other localized factors such as the steric accessibility of the nucleophilic site and the electronic environment surrounding the site may also have an effect. ${ }^{49}$ Further detail regarding the formation of protein adducts is discussed in Section 2.3

In order for a biomarker of exposure to be useful, several key characteristics need to be met. ${ }^{52}$ First (and most importantly in terms of forensic applications), the biomarker should be specific to the xenobiotic in question. While the use of metabolites as biomarkers of exposure is arguably a simpler method of detection, metabolites do not always meet this first characteristic. For example, nitrogen mustards rapidly hydrolyze to ethanolamines in aqueous solutions (such as blood), and are thus the major metabolites seen both in plasma and excreted in urine. However, these metabolites are not specific to $\mathrm{HN}$ exposure, as ethanolamines themselves may be used for industrial or personal applications. ${ }^{53}$ For this reason, a different biomarker of exposure should be selected for CWA HN exposure determination. This uniqueness criterion may be satisfied by utilizing protein or DNA adducts as exposure biomarkers, because such adducts would not form in the absence of the xenobiotic. In addition, not all metabolites are reactive and therefore may not adduct to nucleophilic sites; therefore, this may add a level of specificity for the compound of interest if it has a reactive metabolite capable of macromolecular adduction. However, caution must be utilized, as drugs with similar structures may form the same reactive metabolites.

The second key characteristic for an ideal biomarker of exposure is a relatively long lifetime in the body. This is important in cases where the time between exposure and sample 
collection or analysis is long. While some xenobiotics may have long-circulating metabolites, this is problematic when using metabolites as biomarkers of exposure for HN. As these compounds hydrolyze so quickly, they are subsequently rapidly excreted from the body as a result of an increase in polarity of the compound.

The final characteristic for an ideal biomarker is that it should demonstrate stability in biological matrices. Related to the second listed characteristic, stability allows for a more reliable marker over time and when analyzing samples from multiple individuals. This point is important when considering DNA adducts as potential biomarkers of exposure. Due to the critical importance of DNA in living systems, several highly efficient repair mechanisms exist to ensure that the integrity of DNA remains intact. Xenobiotic adduction of DNA may therefore trigger these repair mechanisms, thus making these biomarkers potentially less stable than protein adducts, where repair mechanisms are minimal or nonexistent. ${ }^{54}$

Based on the abovementioned criteria, protein adducts can therefore provide a specific biomarker of exposure for a xenobiotic of interest. Ideally, adducts to a target protein for the particular xenobiotic would produce the most sensitive biomarker; however, in the case that the target protein is unknown or inaccessible, other proteins may be utilized. Two of the most common proteins employed for biomonitoring are hemoglobin $(\mathrm{Hb})$ and human serum albumin (HSA). These two proteins are well suited as biomonitors because of several favorable characteristics. First, blood is relatively easily obtained from individuals. Second, these two proteins are highly abundant in blood - one $\mathrm{mL}$ of blood contains approximately $150 \mathrm{mg}$ of $\mathrm{Hb}$ and $30 \mathrm{mg}$ of HSA. In contrast, in this same $\mathrm{mL}$ of blood, only a small amount of DNA is present. ${ }^{54}$ Finally, both $\mathrm{Hb}$ and HSA have known kinetic rates of turnover in the body $-\mathrm{Hb}$ circulates in the bloodstream for approximately four months, and HSA has a half-life of approximately 20 days in humans. ${ }^{55}$ Because of their long circulation times, adducts to these 
proteins have the potential to be detected much longer after exposure than parent compounds or circulating metabolites.

\subsection{Protein Adduction Mechanism}

The formation of protein adducts depends on several factors; however, it ultimately comes down to the relative nucleophilicity of the amino acid site on the protein and the electrophilic nature of the xenobiotic of interest. Most xenobiotics will undergo some kind of biotransformation (whether passive or enzymatic) to a more reactive electrophilic species. ${ }^{56}$ These electrophilic species may then bind to nucleophilic centers on proteins; however, the reactivity of these sites varies and is affected by several different factors.

The first factor to consider is the local electronic environment of an amino acid in a protein. Not all amino acids are nucleophilic at physiological $\mathrm{pH}$-depending on the $\mathrm{pK}_{\mathrm{a}}$ of the amino acid, some amino acids may be more likely to demonstrate electrophilic qualities (i.e., a positive charge) and therefore not provide a binding site for electrophilic xenobiotics. It is also of importance to note that, while amino acids may have a "base" $\mathrm{pK}_{\mathrm{a}}$ value, it is possible that, depending on both long-range and local electrostatic influences in the protein structure, a lower or higher local $\mathrm{pK}_{\mathrm{a}}$ may occur for a particular residue. ${ }^{57}$ For example, while cysteine (Cys) is considered the softest biological nucleophile and thus one of the most reactive sites for protein adduction, its base $\mathrm{pK}_{\mathrm{a}}$ value is $\sim 8.3$, meaning that at physiological $\mathrm{pH}(7.4)$, the majority of Cys residues would be unionized (i.e., in the thiol form) and therefore not be in the more favorable thiolate form $\left(\mathrm{S}^{-}\right)$for adduction. However, reactive Cys groups in biological proteins often exist in an arrangement where neighboring basic or acidic amino acids facilitate the deprotonation of the thiol to produce the thiolate, which is then susceptible to electrophilic modification. ${ }^{58}$ Other amino acids capable of such $\mathrm{pK}_{\mathrm{a}}$ modification include aspartic acid (Asp), glutamic acid (Glu), histidine (His),lysine (Lys), tyrosine (Tyr), and the protein terminal groups (both the C-terminus

and the N-terminus). ${ }^{57}$ Similar variability of local $\mathrm{pK}_{\mathrm{a}}$ values have been seen with both Lys ${ }^{59}$ and 
$\mathrm{His}^{60}$ residues on a variety of biological proteins. Therefore, despite the base $\mathrm{Lys}_{\mathrm{pK}} \mathrm{a}$ of $\sim 10.4$, these local electronic factors may lower the $\mathrm{pK}_{\mathrm{a}}$ of a residue to the point at which is it uncharged at $\mathrm{pH} 7.4$ and therefore available for adduction by electrophilic species. As the base $\mathrm{pK}_{\mathrm{a}}$ of His is $\sim 6$, these electronic effects are not as significant, as the majority of His side chains are unionized at $\mathrm{pH}$ 7.4; however, it may still play a role in electrophilic adduction.

Second, even if an amino acid is sufficiently nucleophilic, steric factors will also play a role in whether protein adducts will occur. ${ }^{61}$ This applies both to the nucleophilic site and the attacking electrophile. Some electrophilic species may be too bulky to adduct, or a nucleophilic site may be hindered by surrounding amino acids that do not allow for adduction. Hypothesized adduction sites may therefore exhibit enhanced reactivity if the site is not hindered by other amino acids or other components of a protein's structure.

While inherent nucleophilicity and electrophilicity are important for the formation of protein adducts, it is also important to consider a second characteristic of the reacting species. A compound's "hardness" or "softness" is a description of the relative electron density surrounding a reactive site and its overall polarizability. Compounds are classified as generally "soft" when they have a large electron density (as seen with compounds with an extended $\pi$ electron network) and are highly polarizable. Compounds are classified as generally "hard" when they exhibit the opposite characteristics - typically, these compounds will have a relatively small electron density (a centralized location of charge) that is less polarizable. These characteristics are directly related to the outermost orbital energies (the Highest Occupied Molecular Orbital, or HOMO and the Lowest Unoccupied Molecular Orbital, or LUMO) for the respective compounds. ${ }^{62}$ The Hard and Soft Acid-Base (HSAB) theory states that compounds classified as soft are more likely to interact with other soft compounds, and vice versa. ${ }^{51}$ Therefore, predictions as to whether an electrophile and nucleophile will interact favorably can be done using quantum mechanical calculations based on the HOMO and LUMO energies for the nucleophilic and electrophilic species. Equation 1 


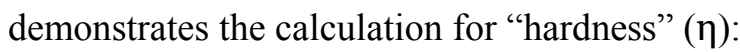

$$
\eta=\frac{\left(E_{L U M O}-E_{H O M O}\right)}{2}
$$

\section{Equation 1: Calculation of hardness. ${ }^{62}$}

The higher the calculated hardness value, the more the species in question can be classified as "hard." Conversely, the lower the calculated hardness value, the more the compound is classified as "soft." It is important to note that there is not a "cutoff" value at which a compound is labeled as "soft" or "hard" - values may be used as a comparative tool between various electrophiles or nucleophiles, but only with experimental data can binding be confirmed. ${ }^{62}$ Hardness can be calculated for both the electrophilic and nucleophilic species of interest. The closer these values are to each other, the more similar those two species are in terms of hardness (and, thus, more likely to react).

The calculated value for hardness may be combined with a second calculated value known as chemical potential $(\mu)$, which describes a molecule's ability to transfer electron density to a different molecule. This characteristic can therefore be used to describe a compounds inherent nucleophilicity - the larger the calculated chemical potential, the better a compound is at transferring electron density, and therefore, the stronger its nucleophilic qualities. Chemical potential is also calculated using the HOMO and LUMO energies for a compound of interest (Equation 2):

$$
\mu=\frac{\left(E_{L U M O}+E_{H O M O}\right)}{2}
$$

\section{Equation 2: Calculation for chemical potential. ${ }^{62}$}

As previously stated, the two most important factors for predicting whether two species will form covalent bonds are the nucleophilicity/electrophilicity of the reacting species (as calculated by Equation 2 above), and their relative hardness or softness (as calculated by Equation 1 above). As such, a third equation may be utilized that combines the calculated 
hardness and chemical potential for a nucleophile and electrophile of interest to produce a "Reactivity Index" $\left(\omega^{-}\right),{ }^{63}$ as seen in Equation 3:

$$
\omega^{-}=\frac{\eta_{A}\left(\mu_{A}-\mu_{B}\right)^{2}}{2\left(\eta_{A}+\eta_{B}\right)^{2}}
$$

Equation 3: Calculation for reactivity index, where $A=$ nucleophile and $B=$ electrophile.

The Reactivity Index can be utilized to compare the predicted reactivity of several electrophiles to a nucleophile of interest, and has demonstrated good agreement with experimental determinations of nucleophile-electrophile interactions. ${ }^{62}$

Values for the HOMO and LUMO energies for selected electrophilic and nucleophilic species may be obtained through the use of quantum mechanical software that will calculate these energies based on the lowest energy conformation of a molecular structure. A variety of theoretical models exist for the modeling of a compound's molecular orbitals; however, one of the most commonly used models is density functional theory (DFT), specifically the B3LYP model, which provides consistent and efficient results for whole-molecule modeling of most compounds. $^{64}$

A final consideration for protein adduction involves the function of the amino acid residue to be adducted, and the function of the adducted protein in general. For example, the functions of proteins such as metallothionein is to act as scavenger proteins and trap reactive species (such as free radicals and metals) within the body. ${ }^{65}$ Therefore, identification of metallothionein-based protein adducts may be quite useful, and such adducts might be prevalent after xenobiotic exposure due to its cysteine-rich structure; however, their short circulation time in the body may limit their usefulness as long-term biomarkers. ${ }^{66}$ In contrast, a protein such as hemoglobin has a specific function (oxygen transport) that does not include detoxification. ${ }^{67}$ An adduct that interferes with this function is highly unfavorable, and while some modifications may be repaired, those that cannot would most likely result in the adducted $\mathrm{Hb}$ being destroyed. ${ }^{68}$ 
These adducts would therefore be less stable (and as a consequence, not a reliable exposure biomarker) than adducts which do not alter the function of the protein. However, should xenobiotic adduction occur on $\mathrm{Hb}$ and not alter its oxygen transport function, it has the potential to remain on the protein during its lifetime in the blood.

\subsection{Protein Adduction by Chemical Warfare Agents}

Previous research regarding protein adduction of CWA has generally been determined based on the known active sites of these agents. In the case of the OP nerve agents, adducts to $\mathrm{AChE}^{8,69,70}$ and $\mathrm{BuChE}^{20,21,71}$ are commonly used. As these proteins are considered the main targets of nerve agents, such adducts make for sensitive exposure biomarkers and have been detected in a number of instances with both in vitro and in vivo exposure. However, there have been other protein adduct biomarkers identified for these agents. Williams et. al ${ }^{72}$ and others ${ }^{73-75}$ identified adduction of sarin, soman, tabun, and VX to a Tyr residue (Tyr-411) on HSA. These adducts were identified as stable for up to a week after subcutaneous exposure to guinea pigs and did not appear to be affected by oxime (2-PAM) therapy. This adduction site is not unique to the CWA organophosphates - adduction to Tyr-411 has also been identified with chlorpyrifos, a common OP pesticide used in agriculture. ${ }^{76}$ The identification of OP protein adducts has been done in a variety of ways, most commonly of which involves the regeneration of the active Ser and subsequent release of the nerve agent(s) for analysis via GC-MS. ${ }^{16,18,19,77,78}$ More classic proteomic techniques involve the use of a digestive enzyme such as trypsin, ${ }^{72}$ chymotrypsin, ${ }^{20}$ or pronase $^{74}$ for isolation of a peptide containing the adducted residue and subsequent analysis via LC-MS and liquid chromatography tandem mass spectrometry (LC-MS-MS).

Adducts to blister agents, most research of which has focused on HD, are diverse in terms of protein targets, as the critical protein target(s) of these agents have yet to be identified. HD adducts have been characterized on the Cys-34 residue of HSA, ${ }^{79-81}$ the N-terminal Val of $\mathrm{Hb},{ }^{82-}$ ${ }^{85}$ keratin, ${ }^{86,87}$ keratinocytes, ${ }^{88}$ glutathione,${ }^{89}$ metallothionein, ${ }^{90}$ and cell membrane proteins such 
as actin. ${ }^{27}$ Additional $\mathrm{HD}$ protein adduction sites on $\mathrm{Hb}$ included His, Glu, and Asp. It was determined that His adduction was most prominent; however, adducts could not be sensitively analyzed given the methodology used (GC-MS) in these earlier studies. ${ }^{83,84}$ The identification of Cys-34 adduction has been widely done with pronase digestion and characterization of the adducted tri-peptide cysteine-proline-phenylalanine (Cys-Pro-Phe) via LC-MS-MS analysis. Despite pronase's general low specificity in terms of protein digestion, identification of the reaction site was made possible via a comparison to a synthesized tri-peptide standard and use of radiolabeled $\mathrm{HD} .{ }^{80,91}$ Identification of $\mathrm{N}$-terminal adduction of $\mathrm{Hb}$ was performed via a modified Edman Degradation process, in which the classical Edman reagent (phenyl isothiocyanate) was replaced with a pentafluoro derivative (pentafluorophenyl isothiocyanate), allowing for the selective removal of the modified valine (Val) residue..$^{92}$

Protein adduct research for the nitrogen mustard family has been less extensive than that for the OP nerve agents and HD; however, it has followed in the same vein as its blister agent relative. Protein adducts to nitrogen mustard CWA (mostly HN-2) have been identified on Cys-34 of $\mathrm{HSA},{ }^{93} \mathrm{~N}$-terminal Val of $\mathrm{Hb},{ }^{94}$ glutathione, ${ }^{41}$ and metallothionein. ${ }^{95}$ However, most research regarding $\mathrm{HN}$ has been focused on detection of DNA adduction and cross-links, ${ }^{24,25,96}$ which has led to the development of multiple drugs containing the nitrogen mustard moiety (further discussed in sections 2.6 and 2.7 regarding therapeutic $\mathrm{HN}$ compounds and in vitro metabolism, respectively). Available methods for $\mathrm{HN}$ adduction determination are similar to those seen with HD.

Considering the significant physicochemical similarities between HD and the nitrogen mustard agents, it is expected that the HN CWA would adduct to similar sites as seen with HD. However, the nitrogen center (as compared to the sulfur center) exhibits some "harder" electrophilic qualities that may allow for nitrogen mustards to bind to a wider variety of nucleophilic centers, most notable Lys and His, which are moderately hard nucleophiles and 
therefore may align well with $\mathrm{HN}$ reactivity. ${ }^{97}$ These could represent important and largely unexplored alternative adduction sites. Consequently, further research is needed to identify additional, potentially more specific and reliable exposure biomarkers of HN CWA based on protein adducts.

\subsection{Bottom-Up Proteomics}

In general, mass spectrometry (MS) based proteomic analysis can be split into two different classes of analysis which are dependent on the approach an analyst uses to work with the protein in question. The first method, known as "top-down" proteomics, starts with a whole protein introduced into a mass spectrometer, which is then fragmented in the collision cell into peptides. The masses of these peptides are then matched using bioinformatics tools to identify the protein. The second method, known as "bottom-up" proteomics, utilizes enzymatic cleavage of a full protein prior to its introduction into the mass spectrometer. Tandem mass spectrometry (MSMS) is utilized to fragment enzymatically cleaved peptides, and the resultant ions analyzed (again using bioinformatics approaches) to elucidate an amino acid sequence for each peptide (Figure 5). This method is most typically used for the identification of post-translational modifications, particularly when the modification creates a predictable mass shift that can be observed via MS or MS-MS. For this reason, bottom-up proteomics is very useful for the determination of xenobiotic adduction sites on proteins, as they also create a mass difference between the native peptide mass and the adducted peptide mass. If the mass increase is well characterized, adduction sites can be identified with high confidence. 

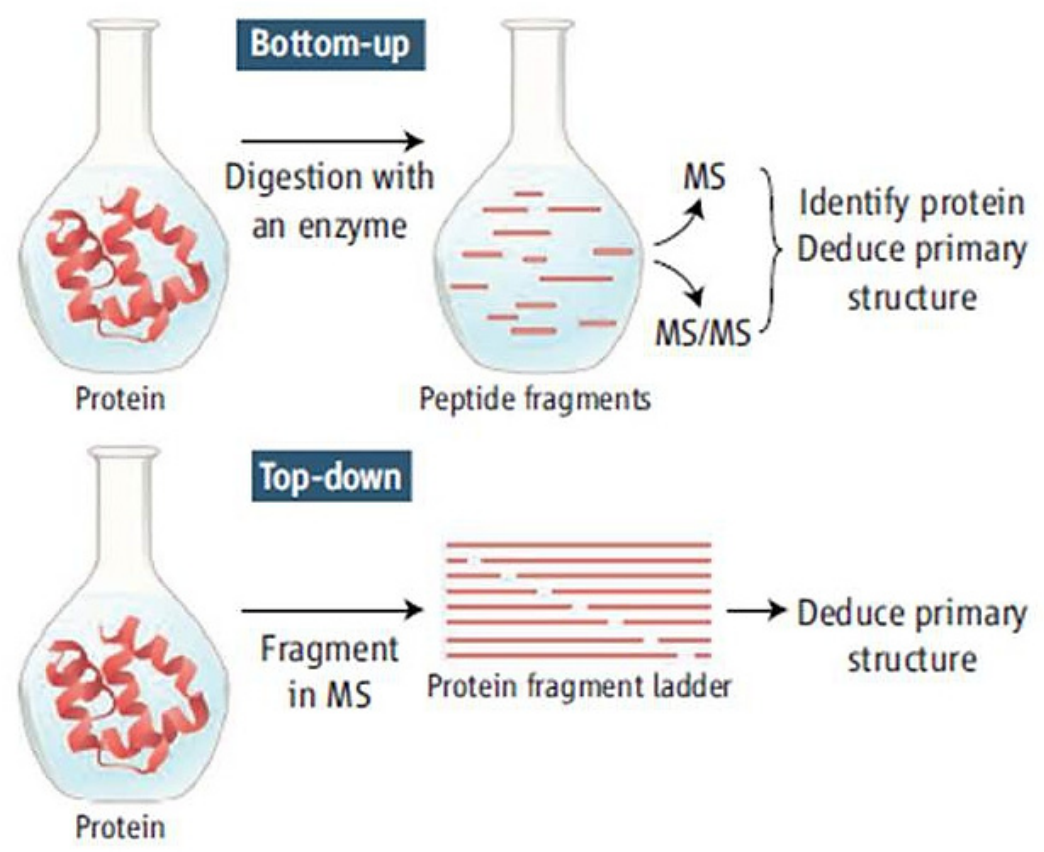

Figure 5: Bottom-up versus top-down proteomics. ${ }^{98}$ Bottom-up analysis involves enzymatic digestion of the protein prior to MS analysis, whereas top-down analysis utilizes the fragmentation capabilities of the MS for identification.

When peptides are introduced into an MS collision cell, they tend to fragment in a very specific manner, which is quite useful when analyzing an MS-MS spectrum to elicit amino acid composition and sequence. Figure $6^{99}$ demonstrates the typical fragments observed with peptide MS-MS, including conventional descriptors for each fragment.

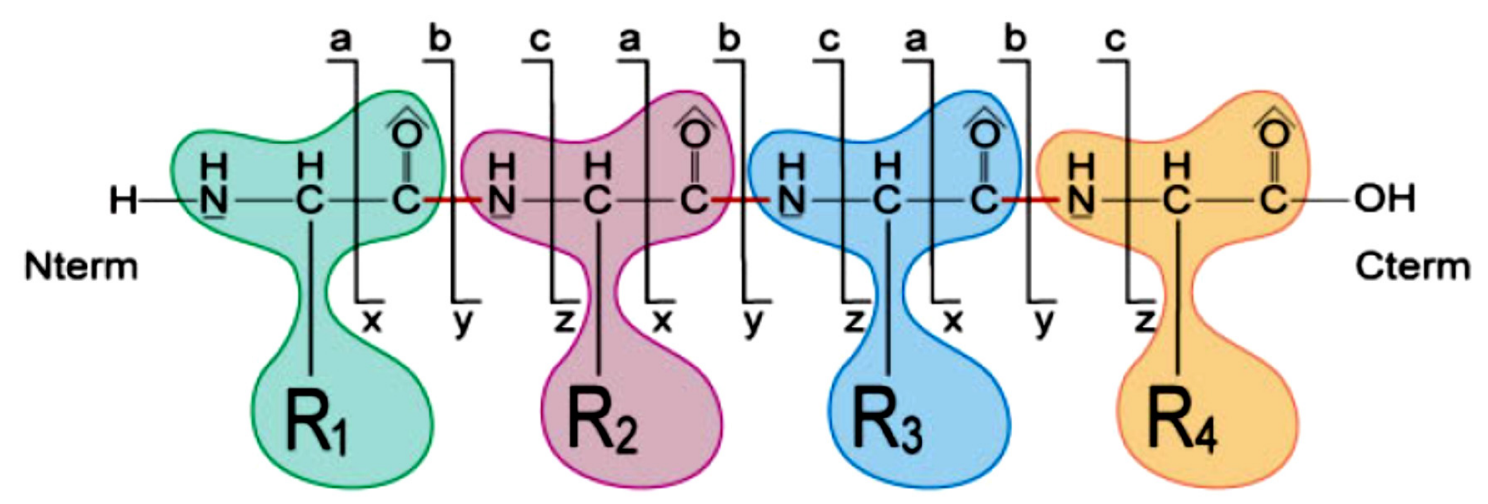

Figure 6: Description of peptide MS-MS fragments. Typical fragmentation results in formation of $b$ and $y$ ions; however, $a$ and $x$ fragments may also be encountered based on collision energy parameters. ${ }^{99}$ 
Bottom-up proteomics is also quite useful when the protein in question is well characterized (i.e., containing a known amino acid sequence) and when an enzyme with wellcharacterized cleavage sites is utilized for digestion. Typically, trypsin is used, a serine protease that cleaves at the carboxy-terminus of Lys and arginine (Arg) residues, except when followed by a Pro. Trypsin has a high level of specificity, making it an excellent choice for a predictable digestion enzyme. ${ }^{100}$ However, should a protein of interest contain few (or too many) Lys or Arg residues, trypsin would not be well suited as it would form large (or very small) fragments that are more difficult to analyze. Therefore, other enzymes are also available for protein digestion, and can be utilized based on experimental requirements and conditions.

A variety of software packages may be used to assist in the identification of posttranslational modifications or xenobiotic adducts. These programs utilize the obtained peptide mass for both MS and MS-MS data and match it to potential iterations of a known peptide (i.e., found via a database or in-house specification of protein and peptide sequences) or a peptide with a modification of some kind. Because of this matching process, it is important to collect highly accurate MS and MS-MS data so as to provide a high confidence match to identify individual peptides and, thus, the starting protein. Therefore, data collection should be done on a high-mass accuracy instrument for best results. The use of MS-MS based approaches for proteomic data collection and analysis has offered great improvements over previous proteomic approaches such as gel electrophoresis. As mentioned above, it is important to consider software specification when determining which mass analyzer to utilize. However, it is also important to consider the advantages and disadvantages of each MS system, and use these qualities wisely to obtain the desired results.

The first consideration is the type of ionization required for the analysis of choice. As the majority of bottom-up proteomics involves multiple peptides formed after enzymatic digestion, it 
is highly desirable to incorporate some type of chromatographic separation for improved analysis. As peptides are non-volatile analytes, liquid chromatography (LC) is the separation technique of choice and, as such, an ionization technique such as electrospray ionization (ESI) is most commonly used, as it is easily coupled to LC. ${ }^{101}$ Electrospray ionization is an atmospheric pressure ionization technique in which a liquid (specifically, the LC effluent) is introduced into a charged capillary, which is held at a high potential (typically positive) as compared to a ground. This charge differential creates the formation of a Taylor cone at the tip of the capillary as the effluent travels out the capillary. Charged droplets are released from the Taylor cone and desolvated in the high temperature source, leaving behind charged peptide ions that are subsequently introduced into a mass analyzer (Figure 7). ${ }^{102}$ While it is also commonplace to use other ionization sources for proteomic analysis (such as Matrix-Assisted Laser Desorption Ionization, or MALDI), the ease with which ESI ionization can be coupled to LC allows for analysis of chromatographic effluent that could only be achieved with MALDI via fractionation and analysis of each fraction individually. In addition, the common production of multiplycharged species in ESI (MALDI typically produces singly charged ions) may allow for the analysis of larger peptides even when a mass analyzer has a relatively narrow $\mathrm{m} / \mathrm{z}$ range. ${ }^{101}$ 


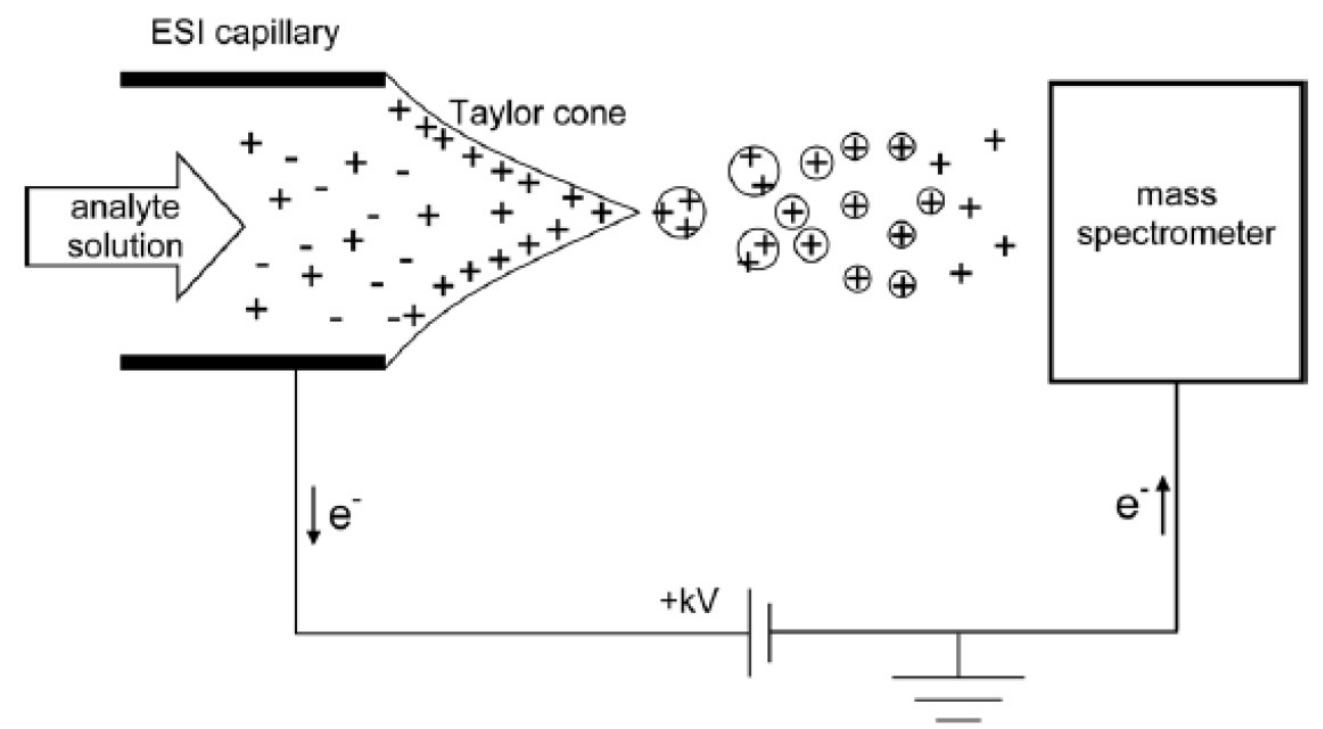

Figure 7: Positive model electrospray ionization (ESI). ${ }^{102}$ The analyte solution is given a charge through the capillary, creating highly charged droplets that are desolvated before entering the mass spectrometer.

The second important item to consider is the type of mass analyzer and its suitability for a specific proteomic application. While there are several mass analyzers to consider, this review will focus on the two analyzers utilized during the present research - triple quadrupole (QQQ) and quadrupole-time-of-flight (Q-TOF) analyzers.

The QQQ analyzer is a highly versatile mass analyzer that is composed of three quadrupoles in tandem, with the center quadrupole acting as a collision cell. This arrangement of analyzers in either a fixed or scanning mode can allow for a variety of MS and MS-MS experiments, including MS Scan, Single-Ion Monitoring (SIM), Product Ion Scan, Neutral Loss Scan, and Multiple Reaction Monitoring (MRM) (Figure 8). Of specific interest to proteomic research is the MS-MS capabilities of the instrument (Product Ion Scan, Neutral Loss Scan, and MRM). These types of MS-MS experiments can allow for the analysis of peptides that contain similar sequences or modifications while minimizing unwanted data. In addition, MRM can allow for a highly selective and sensitive method for detecting a known peptide of interest. ${ }^{103}$ However, 
QQQ analyzers do not produce high mass accuracy data, which are essential when attempting to identify unknown peptides or modifications. Triple quadrupole analysis is therefore best suited when the peptides and modifications are well known so as to take advantage of its high selectivity and sensitivity.

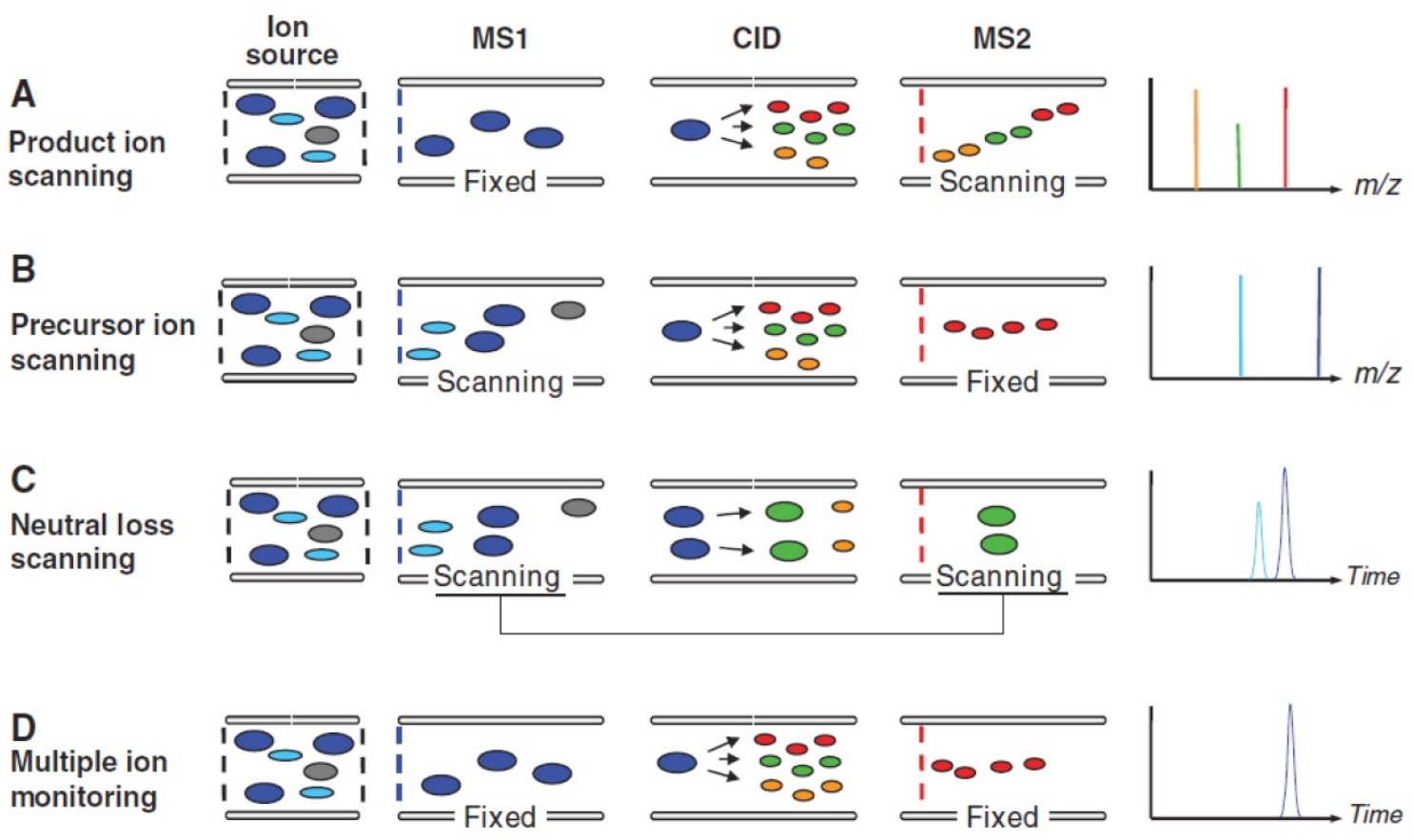

Figure 8: Various modes for QQQ analysis. ${ }^{103}$ Multiple Reaction Monitoring is a highly selective and sensitive method that may be used in proteomic analysis once post-translational modifications have been successfully identified.

The Q-TOF mass analyzer is capable of producing high mass accuracy data due to the time-of-flight (TOF) analyzer. Ions are "pushed" into a flight tube under vacuum, in which all ions are given the same kinetic energy entering the tube. Ions will travel through the tube and reach the detector at a speed that is inversely proportional to the mass-to-charge $(\mathrm{m} / \mathrm{z})$ ratio of the ion (Figure 9). ${ }^{104}$ The incorporation of the quadrupole at the front end and a collision cell between the two analyzers allows for MS-MS analysis that produces high mass accuracy product ions, which is necessary for accurate database matching of unknown peptides. In addition, the accurate mass data is also helpful when attempting to identify protein modifications, both known and 
unknown. However, Q-TOF instruments are not capable of the same sensitivity that can be achieved using MRM mode on a QQQ instrument. ${ }^{103}$ Nevertheless, these two instruments may be used to complement each other - Q-TOF analysis can be done to identify a peptide of interest, and QQQ analysis may be done to increase the sensitivity of its detection once the peptide has been identified and characterized.

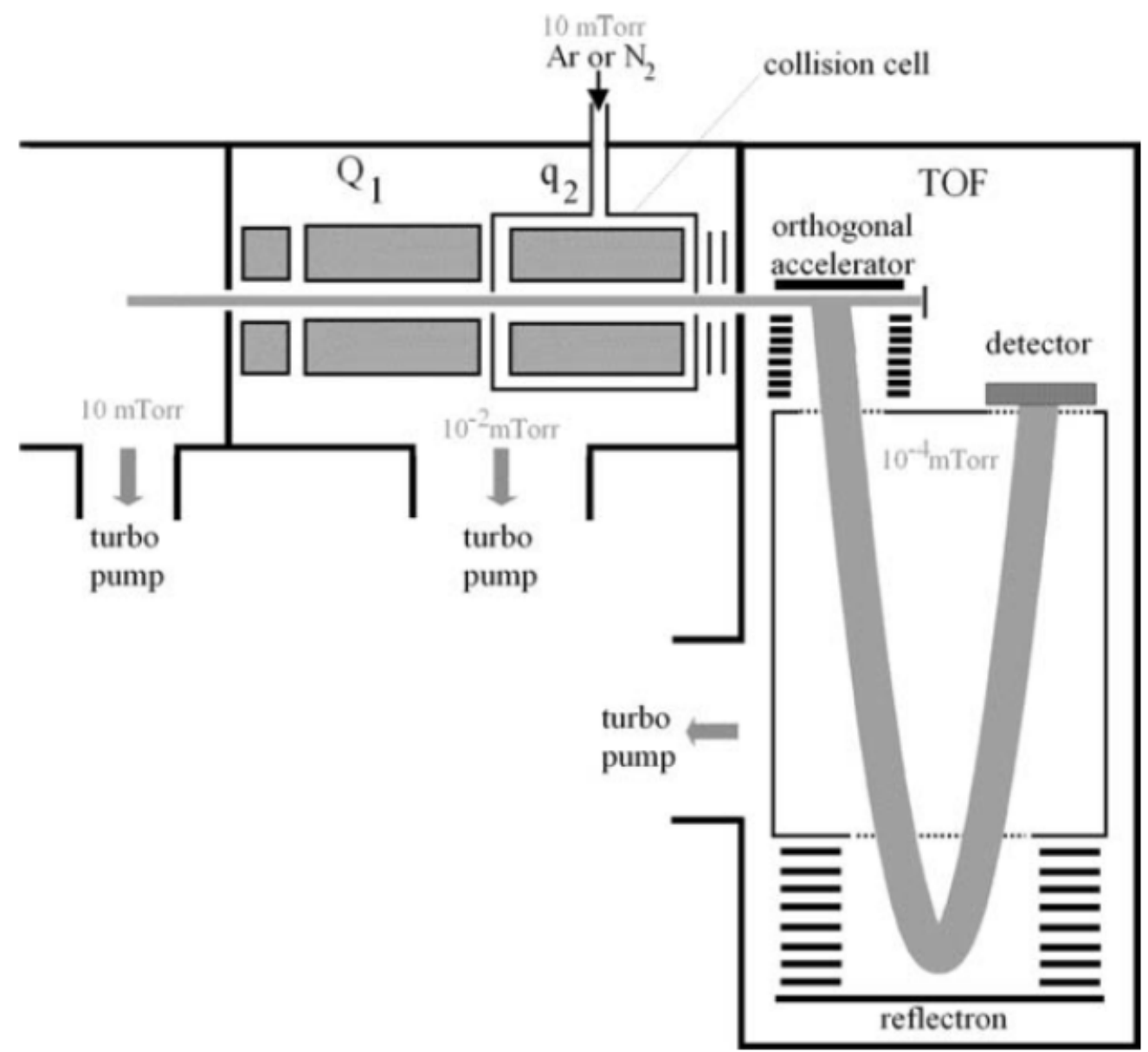

Figure 9: Schematic of quadrupole-time-of-flight (Q-TOF) mass analyzer. ${ }^{104}$ The Q-TOF is particularly useful in proteomics when a modification has not yet been positively identified due to its ability to produce high mass accuracy data.

\subsection{Nitrogen Mustard Therapeutic Agents}

Due to the success of HD as a chemical weapon, research began after WWI to develop an analogue of HD as a more efficient CWA. As nitrogen mustards were being investigated, it was observed that administration of these compounds could treat a variety of cancers, most notably 
leukemia. ${ }^{2}$ As such, therapeutic agents containing these nitrogen mustard moieties were developed as cancer treatments.

As mentioned previously, known adduction sites for electrophilic species include sites on DNA bases, most notably the N-7 position of guanine. When adducted to DNA, HN compounds are able to form a variety of mono- and bi-alkylated products, including DNA intra- and interstrand crosslinks, and DNA-protein crosslinks. ${ }^{105}$ When this occurs in rapidly dividing cells (as is the case with cancer), this cross-linking can induce apoptosis, thus treating the disease. ${ }^{106}$ However, this cross-linking is also associated with cytotoxicity in healthy cells; ${ }^{107}$ as such, development of drugs with a higher therapeutic index was necessary for a more targeted approach to the cancer cells over healthy cells. For example, pro-drugs such as cyclophosphamide and ifosphamide were designed to contain a phosphate bond that could be cleaved in cells with high phosphoramidase activity (as in certain gastric cancers), producing an active phosphoramide mustard that could either react itself, or be further metabolized to nornitrogen mustard. ${ }^{108-110}$ More recently, dinitrobenzamide mustards have been developed that require activation via nitroreduction, produced by tumor cells. ${ }^{111-113}$ Other drugs, such as chlorambucil and melphalan, can adduct DNA directly without the need of metabolic activation. ${ }^{46,106}$ Figure 10 shows the structure of some of the more common nitrogen mustard based therapeutic compounds. The most common HN-containing therapeutic drugs currently utilized are cyclophosphamide (CP), chlorambucil (CB), and melphalan (MP). These drugs are capable of treating a wide variety of cancers, including leukemia $(\mathrm{CP}, \mathrm{CB})$, lymphoma $(\mathrm{CP}, \mathrm{CB})$, ovarian cancer $(\mathrm{CP}, \mathrm{CB}, \mathrm{MP})$, breast cancer $(\mathrm{CP}, \mathrm{CB}, \mathrm{MP})$, myeloma $(\mathrm{MP})$, lung cancer $(\mathrm{CP})$, and prostate cancer $(\mathrm{CP}) .{ }^{107}$ 
<smiles>CN(CCCl)CCCl</smiles>

Mechlorethamine (HN-2)<smiles>O=P1(CCCl)NCCCO1</smiles>

Cyclophosphamide<smiles>Cn1c(CCCC(=O)O)nc2cc(N(CCCl)CCCl)ccc21</smiles>

Bendamustine<smiles>NP(=O)(O)N(CCCl)CCCl</smiles>

Phosphoramide Mustard<smiles>O=P1(NCCCl)OCCCN1CCCl</smiles>

Ifosfamide<smiles>O=c1[nH]cc(N(CCCl)CCCl)c(=O)[nH]1</smiles>

Uramustine<smiles>O=C(O)CCCc1ccc(N(CCCl)CCCl)cc1</smiles>

Chlorambucil<smiles>NC(Cc1ccc(N(CCCl)CCCl)cc1)C(=O)O</smiles>

Melphalan<smiles>CC12CCC3c4ccc(OC(=O)N(CCCl)CCCl)cc4CCC3C1CCC2O</smiles>

Estramustine

Figure 10: Structures of common therapeutic drugs containing the nitrogen mustard moiety.

Nitrogen mustard therapeutic compounds have demonstrated their ability to form DNA adducts and DNA cross-links at a variety of nucleophilic sites on DNA. ${ }^{24,46,114}$ In addition to binding to DNA, these compounds may also bind to the DNA repair protein $\mathrm{O}^{6}$-alkyguanine DNA alkyltransferase (AGT) and form DNA-protein cross-links, hindering AGT function and thus representing an alternative mechanism for these compounds. ${ }^{115}$ Resistance to these drugs is typically correlated with increased GSH and glutathione-S-transferase (GST) levels. ${ }^{107} \mathrm{CP}, \mathrm{CB}$, and MP have all demonstrated the ability to form covalent bonds with GSH, ${ }^{116-119}$ which can act as a detoxification mechanism for these drugs. ${ }^{44}$ 


\subsection{In Vitro Metabolic Assays}

In vitro metabolic assays are commonly used in drug discovery research as a way to assess the potential efficacy or toxicity of a new drug. The drug discovery process is both timeconsuming and quite costly, and these metabolic assays can assist in determining whether potential lead drugs are worth continuing to animal or clinical trials. Of largest concern is the metabolism of new drugs that may form reactive metabolites with deleterious effects. These in vitro assays can provide researchers insight into the potential formation of toxic metabolites. ${ }^{120}$

The purpose of metabolism in the body is to transform drugs and other xenobiotics into more water-soluble compounds to facilitate their excretion from the body. Phase I metabolism typically involves oxidation, reduction, or hydrolysis reactions on the parent drug. During this process, reactive metabolites may form which, instead of being passively excreted, will interact with proteins or DNA and cause adverse reactions. The focus of most Phase I metabolism research has centered around the cytochrome P450 (CYP450) isoforms, as they account for approximately half of the metabolic enzymes responsible for drug metabolism. ${ }^{121}$ However, other enzymes, such as monoamine oxidases (MAO) and flavin-containing monooxygenases (FMO), are also quite common in Phase I metabolism. Phase II metabolism, or conjugation reactions, typically involve the addition of a large polar molecule (such as glucuronic acid) to a metabolite (or parent compound) to enhance excretion of compounds from the body. While many drugs typically undergo Phase I metabolism before Phase II metabolism, some drugs are primarily cleared from the body via conjugation. ${ }^{122}$ Figure 11 depicts the variety of mechanisms by which drugs can interact within the body. 


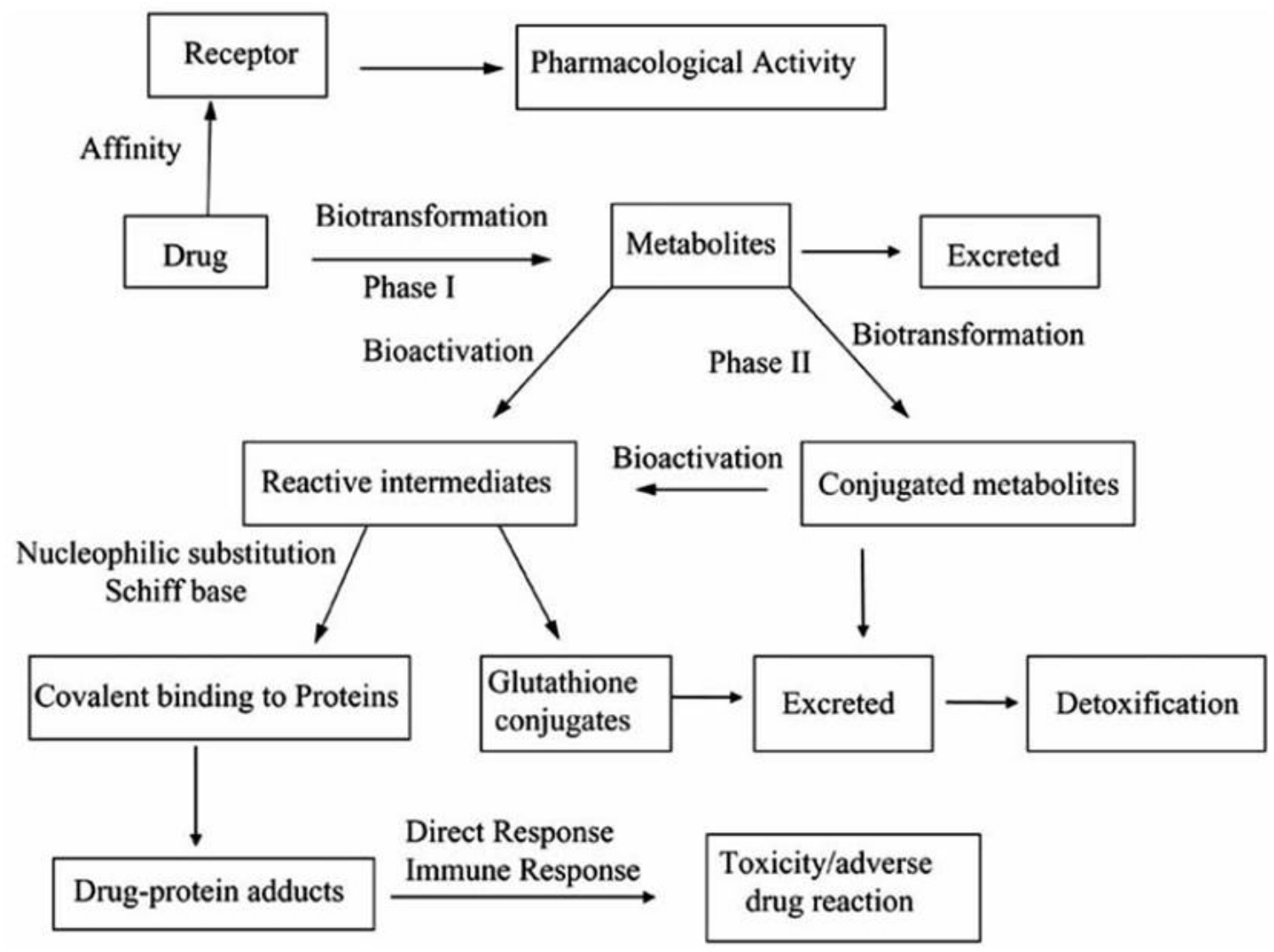

Figure 11: Fate of drugs and xenobiotics in the body. ${ }^{120}$ Compounds may undergo various different metabolic activation processes, which have the potential to form reactive intermediates able to bind to proteins and/or DNA.

One of the most commonly used in vitro metabolic assays for metabolite discovery involves the use of liver homogenate fractions as a medium for metabolism, the most common of which are the cytosolic fraction, the S9 fraction, and the microsome fraction. These fractions are obtained via the low speed centrifugation of a liver homogenate to obtain the S9 fraction (supernatant), which can be further centrifuged at high speed to obtain the cytosolic (supernatant) and the microsomal fraction (pellet). The microsomal fraction is one of the most popular in vitro models, and can provide a good prediction of hepatic CYP450 metabolism. Additionally, the S9 fraction is quite useful due to its ability to produce both Phase I and Phase II metabolites. ${ }^{123}$ The ability of these liver homogenate fractions to mimic metabolic functions that typically occur in 
living tissue involves the use of multiple cofactors that are normally present within tissues. The inclusion or exclusion of certain cofactors can allow for the manipulation of these fractions to perform Phase I or Phase II metabolism, or for experimental controls.

Additional metabolic information may be elucidated by these metabolic systems with the use of nucleophilic agents such as glutathione to "trap" reactive metabolites that may not last long enough in the system to be otherwise detected. Other trapping agents, including short model peptides, may also be utilized to capture a wider variety of electrophilic species that may demonstrate a preference towards a nucleophile other than Cys. ${ }^{124}$

\subsection{Research Objectives}

This research was performed to test three main hypotheses:

Hypothesis I: Nitrogen mustard chemical warfare agents are capable of forming stable adducts with lysine and histidine residues on model peptides and whole proteins.

Hypothesis II: Identified adducts are specific to the chemical warfare agents and can be distinguished from potential adducts formed by the most common nitrogen mustard-containing therapeutic drugs.

Hypothesis III: Identified adducts are stable over an extended period of time and thus are potential biomarkers for nitrogen mustard chemical warfare exposure.

The goal of this project is to identify novel, specific, and stable protein adduct biomarkers of exposure to the chemical warfare agents HN-2 and HN-3. This was accomplished by completing the four tasks detailed below.

Task 1: Determine the adduction potential of HN-2 and HN-3 to model peptides containing the reactive nucleophiles Cys, Lys, and His. Specifically: 
- Determination of the relative kinetics of adduction of HN-2 and HN-3 to the model peptides over an eight $h$ exposure period.

- Determination of an optimal incubation time for $\mathrm{HN}-2$ and $\mathrm{HN}-3$ adduction for later experiments.

- Determination of the stability of identified adducts over a three-week incubation period at physiological conditions $\left(\mathrm{pH} 7.4,37^{\circ} \mathrm{C}\right)$.

- Assessment of the necessary concentration or molar excess of $\mathrm{HN}-2$ or $\mathrm{HN}-3$ required for observable adduction via liquid chromatography triple quadrupole mass spectrometry (LC-QQQ-MS) analysis.

Task 2: Identify sites of adducted Lys and His residues on purified $\mathrm{Hb}$ and HSA after in vitro exposure to HN-2 and HN-3. Specifically:

- Determination of a reproducible digestion protocol for $\mathrm{Hb}$ and HSA for peptide analysis via liquid chromatography quadrupole time-of-flight mass spectrometry (LC-Q-TOFMS) and liquid chromatography quadrupole time-of-flight tandem mass spectrometry (LC-Q-TOF-MS-MS).

- Identification and characterization of Lys and His adducts that occur reproducibly with sufficient MS-MS support.

- Determination of the stability of identified protein adducts over a three-week incubation period at physiological conditions.

Task 3: Perform in vitro human whole blood incubations with $\mathrm{HN}-2$ and $\mathrm{HN}-3$ and determine whether adducts identified in Task 2 are present after protein isolation, purification, and trypsin digestion. Specifically:

- Optimization of protein precipitation conditions for $\mathrm{Hb}$ and HSA isolation from human whole blood. 
- Identification of novel Lys and His adducts on $\mathrm{Hb}$ and HSA (identified in Task 2) via MS-MS support and retention time matching.

Task 4: Use in vitro metabolic systems to assess biotransformation and adduction of three of the most common therapeutic nitrogen mustard therapeutic drugs. Specifically:

- Use model peptides containing Cys, Lys, and His as trapping agents for reactive metabolites formed in the in vitro system for each drug.

- Determine whether, after metabolism, the therapeutic drugs will form the same identified adducts as $\mathrm{HN}-2$ and $\mathrm{HN}-3$.

A fifth task was also completed using quantum chemical calculations to provide additional support for the findings from Tasks 1-3, in addition to providing avenues for future research. Task 5: Use molecular modeling software and quantum mechanical calculations to determine hardness, chemical potential, and reactivity index for relevant nucleophiles and electrophiles from this work. Specifically:

- Compare hardness and chemical potential values of relevant electrophilic species for HN2 and $\mathrm{HN}-3$ to those of a known reactive electrophile.

- Compare hardness and chemical potential values of relevant amino acid species for prediction of reactivity to $\mathrm{HN}-2$ and $\mathrm{HN}-3$ based on experimental data.

- Compare Reactivity Index values for relevant amino acids for specific electrophilic species for prediction of reactivity to $\mathrm{HN}-2$ and $\mathrm{HN}-3$ based on experimental data.

Combined together, these tasks will 1) elucidate novel protein adducts and potential biomarkers of exposure for $\mathrm{HN}-2$ and $\mathrm{HN}-3$ that are specific to the analytes of interest and stable over time, 2) support these findings with quantum mechanical calculations, and 3) provide additional avenues of research for future work. 


\section{METHODOLOGY}

\subsection{Instrumentation}

Sample analysis by LC-QQQ-MS was performed on an Agilent 6460 triple

quadrupole mass spectrometer, while samples analyzed via LC-Q-TOF-MS were performed on an Agilent 6530 quadrupole time of flight mass spectrometer. Both systems were coupled to an Agilent 1290 Infinity ultra-high performance liquid chromatography system utilizing Agilent Jet Stream ESI technology. Chromatographic separation was performed on an Agilent ZORBAX Rapid Resolution HD Eclipse $\mathrm{C}_{18}$ column (100 mm x $2.1 \mathrm{~mm}, 1.8 \mu \mathrm{m}$ particle size), with a column temperature of $40^{\circ} \mathrm{C}$. Solvents utilized for separation consisted of an aqueous phase consisting of $0.1 \%$ trifluoroacetic acid (TFA) in HPLC water and an organic phase consisting of 95:5:0.1\% acetonitrile:HPLC water:TFA. Method specifics for each task may be found in Appendix 6. Data acquisition for both systems was performed using Agilent MassHunter Acquisition software version 6.0 (QQQ) and version 5.0 (Q-TOF). Data analysis was performed using Agilent MassHunter Qualitative Analysis software, with supplementation by Agilent BioConfirm software (version 5.0) for full protein analysis (as seen in Appendix 5).

\subsection{Model Peptide Adduct Screening}

The identification of $\mathrm{HN}-2$ and $\mathrm{HN}-3$ adducts to the model peptides AcPAACAA, AcPAAKAA, and AcPAAHAA (custom synthesized by Biomatik, Inc.) were performed using LC-QQQ-MS. All adduction incubations were performed at $37^{\circ} \mathrm{C}$ in $10 \mathrm{mM}$ sodium phosphate buffer (SPB), pH 7.4, so as to mimic human physiological conditions. Initial adduct identification was done by incubating $0.02 \mathrm{mg} / \mathrm{mL}$ of each peptide with excess $\mathrm{HN}-2$ or $\mathrm{HN}-3$ for $24 \mathrm{~h}$ and analyzed via full scan MS. Confirmation of identified adducts was done using product ion MSMS of the precursor ions identified via the full scan MS method. In addition, each model peptide was incubated with the $\mathrm{HN}-2$ and $\mathrm{HN}-3$ precursors N-methyldiethanolamine and triethanolamine, 
respectively, to determine whether adduction would occur. For these experiments, $0.02 \mathrm{mg} / \mathrm{mL}$ of each peptide was incubated with excess $\mathrm{N}$-methyldiethanolamine or triethanolamine at $37^{\circ} \mathrm{C}$ for $24 \mathrm{~h}$ and analyzed via Full Scan MS. Method specifics for both full scan MS and product ion MSMS may be found in Appendix 6.

Identified adducts were further characterized with regards to kinetics of formation and exposure-response relationships. Kinetics of adduction was assessed by incubating $0.02 \mathrm{mg} / \mathrm{mL}$ each peptide with excess $\mathrm{HN}-2$ or $\mathrm{HN}-3$ and analyzing triplicate samples once every 30 min for 8 h. Exposure-response relationships between $\mathrm{HN}$ concentration and adduction level was performed by $24 \mathrm{~h}$ incubation of triplicate samples of $0.02 \mathrm{mg} / \mathrm{mL}$ model peptide with various molar ratios of HN-2 or HN-3 (i.e., 1:1, 10:1, 25:1, 50:1, 100:1 and 150:1 molar excess HN). Samples were run via full Scan MS (method specifics as seen in Appendix 6).

Finally, adduct stability at $37^{\circ} \mathrm{C}$ was assessed over a three-week analysis period. Triplicate $1 \mathrm{~mL} 0.02 \mathrm{mg} / \mathrm{mL}$ samples were incubated with 100:1 molar excess of HN-2 or HN-3 for $3 \mathrm{~h}$. Following incubation, unreacted $\mathrm{HN}$ was removed from the samples via solid phase extraction (SPE) cleanup using Empore C18 SD cartridges ( $3 \mathrm{~mL}$ ). Cartridges were first conditioned with $1 \mathrm{~mL}$ methanol and equilibrated with $500 \mu \mathrm{L} 0.1 \%$ TFA in 70\% acetonitrile. Samples were then loaded onto the cartridge, washed with $500 \mu \mathrm{L} 0.1 \%$ TFA in HPLC water, and eluted using $500 \mu \mathrm{L} 0.1 \%$ TFA in $70 \%$ acetonitrile. The acetonitrile was evaporated from the samples using a nitrogen blow-down system, leaving approximately $300 \mu \mathrm{L}$ of eluent. Sodium phosphate buffer $(10 \mathrm{mM}, \mathrm{pH} 7.4)$ was added to the samples to bring the volume back to $1 \mathrm{~mL}$. For each time point assessed ( 3 h, 24h, 1 week, 2 weeks, and 3 weeks), an aliquot of each sample was removed, while the remaining sample was kept at $37^{\circ} \mathrm{C}$ during the three-week analysis period. Samples were analyzed via full scan MS (method specifics as seen in Appendix 6). 


\subsection{Full Protein Adduction Studies}

Target proteins $\mathrm{Hb}$ and HSA were analyzed both as intact proteins and via digestion of the protein into peptides so as to identify specific adducted residues. Intact proteins were analyzed via direct injection of the protein (no column separation) using a deconvolution algorithm within the Agilent BioConfirm software package. Deconvolution parameter specifics are seen in Appendix 5. Deconvolution and analysis of intact proteins allowed for not only confirmation of protein molecular weight, but also an indication of approximately how many adducted residues may be present on the proteins after incubation with $\mathrm{HN}-2$ and HN-3. Confirmation of the number of adducted residues, however, was performed using MS-MS analysis of digested proteins.

Full protein digestions were performed as specified in Wisniewski et al. ${ }^{125}$ Sample preparation utilized 10 kilodalton $(\mathrm{kDa})$ cutoff spin filters in order to remove excess reagents, exchange buffer, and to allow for the removal of digested peptides from non-digested protein. Reduction of protein disulfide bonds was performed by adding tris(2-carboxyethyl)phosphine (TCEP) at a final concentration of $50 \mathrm{mM}$ and incubating at room temperature for $1 \mathrm{~h}$. Excess TCEP was removed via centrifugation with the $10 \mathrm{kDa}$ spin filters, followed by cysteine alkylation via addition of iodoacetamide at a final concentration of $50 \mathrm{mM}$. Protein digestion was performed using trypsin at an enzyme:protein ratio of 1:10. Following protein digestion overnight at $37^{\circ} \mathrm{C}, 10 \mathrm{kDa}$ spin filters were utilized to collected digested peptides, while leaving any undigested protein and the digestion enzyme in the spin filter. Collected tryptic peptides were then subject to SPE cleanup using Supelco Discovery DSC-18 SPE cartridges (1 mL). Cartridges were conditioned with $1 \mathrm{~mL}$ methanol and equilibrated with $1 \mathrm{~mL} 0.1 \%$ TFA in $70 \%$ acetonitrile. The collected tryptic peptides were loaded onto the SPE cartridge and washed with $500 \mu \mathrm{L} 0.1 \%$ TFA in HPLC water. Peptides were eluted from the cartridge using $500 \mu \mathrm{L} 0.1 \%$ TFA in $70 \%$ acetonitrile, and a nitrogen blow-down system was used to evaporate the acetonitrile, leaving 
approximately $100 \mu \mathrm{L}$ of eluent. Eluents were analyzed via LC-Q-TOF-MS-MS. Method specifics for $\mathrm{Hb}$ and HSA analysis are as seen in Appendix 6.

Data analysis of the digested proteins was performed using Agilent BioConfirm software package. The software package identifies compounds from the auto MS-MS data, and uses protein sequences added by users to match the sequence to compounds found in the data file, factoring in complete digestion, incomplete digestion, and predicted amino acid modifications. Analysis method specifics for the identification of compounds and sequence matching may be found in Appendix 5. All matched results obtained from BioConfirm were assessed for accuracy based on theoretical MS-MS fragmentation and for reproducibility among multiple samples.

To identify the lowest molar ratio of $\mathrm{HN}$ :protein necessary to produce adduction (and thus, the most reactive site(s) of adduction), protein samples $(30 \mu \mathrm{L} 8 \mathrm{mg} / \mathrm{mL}$ lyophilized protein in $50 \mathrm{mM}$ AmBic, $\mathrm{pH}$ 8.0) were incubated with varying molar excess of $\mathrm{HN}(1: 1.10: 1,25: 1$, $50: 1,100: 1,150: 1$, and 200:1). Samples were allowed to incubate at $37^{\circ} \mathrm{C}$ for $3 \mathrm{~h}$, then excess $\mathrm{HN}$ was removed and samples were concentrated using $10 \mathrm{kDa}$ spin filters. Samples were then analyzed via direct (i.e., column-free) injection analysis on LC-Q-TOF-MS. Peaks were deconvoluted using BioConfirm analysis software in order to identify which concentration ratio would produce a noticeable mass shift corresponding to approximately one adduction by $\mathrm{HN}-2$ or $\mathrm{HN}-3$. This ratio was then utilized in samples that were digested via the protocol described above, and adduction site(s) identified via analysis of sample data using BioConfirm software.

Identification of protein adducts was done by incubating triplicate samples $(30 \mu \mathrm{L} 8$ $\mathrm{mg} / \mathrm{mL}$ lyophilized protein in $50 \mathrm{mM}$ ammonium bicarbonate (AmBic), $\mathrm{pH} 8.0$ ) with $\mathrm{HN}-2$ or $\mathrm{HN}-3(100 \times \mathrm{HN}$ for $\mathrm{Hb}, 50 \times \mathrm{HN}$ for $\mathrm{HSA})$ at $37^{\circ} \mathrm{C}$ for $3 \mathrm{~h}$. Samples placed in $10 \mathrm{kDa}$ spin filters and washed twice with $200 \mu \mathrm{L}$ HPLC water to concentrate the sample and to remove unreacted $\mathrm{HN}$. The sample was split into two aliquots - the first (approximately $10 \mu \mathrm{L}$ ) was diluted to approximately $1 \mathrm{mg} / \mathrm{mL}$ and analyzed via direct injection, and the second (approximately $30 \mu \mathrm{L}$ ) 
was digested to identify specific adducted residues. BioConfirm was utilized to analyze digest samples by matching identified compounds with the protein sequence, allowing for compounds to be complete digest fragments, incomplete digest peptides, or peptides with predicted modifications. Any peptides identified with a predicted modification of $\mathrm{HN}-2$ or $\mathrm{HN}-3$ were confirmed by analyzing MS-MS data produced and comparing the peaks to expected peptide fragmentation as identified by Protein Prospector (http://www.prospector.ucsf.edu) ${ }^{126}$ Identified adducts were determined as true adducts if present in all three triplicates at the same retention time with MS-MS confirmation in at least two of the three samples (adduct identification criteria further discussed in Section 4.2).

Finally, the stability of identified adducts was performed over a three-week analysis period. Seven samples consisting of $200 \mu \mathrm{L} 8 \mathrm{mg} / \mathrm{mL}$ lyophilized protein (HSA or $\mathrm{Hb}$ ) were utilized for these experiments. For $\mathrm{Hb}$ stability, triplicate samples of $\mathrm{Hb}$ were incubated with 200 $\mu \mathrm{L} \mathrm{HN}-2$ or $\mathrm{HN}-3$ for a final concentration of 100:1 molar excess $\mathrm{HN}$ (as compared to the protein concentration) and one control protein sample incubated with $200 \mu \mathrm{L} 50 \mathrm{mM}$ AmBic, pH 8.0. For HSA, triplicate samples of protein were incubated with $200 \mu \mathrm{L} \mathrm{HN}-2$ or HN-3 for a final concentration of 50:1 molar excess $\mathrm{HN}$ (as compared to the protein concentration), with a final sample serving as a protein control, incubated with $200 \mu \mathrm{L} 50 \mathrm{mM}$ AmBic, pH 8.0. Protein samples were incubated for $3 \mathrm{~h}$ at $37^{\circ} \mathrm{C}$, followed by excess $\mathrm{HN}$ removal and sample concentration via $10 \mathrm{kDa}$ spin filters. AmBic $(50 \mathrm{mM}, \mathrm{pH} 8.0)$ was utilized to bring the recovered protein volume back to $200 \mu \mathrm{L}$ after cleanup. To each sample, $1 \mu \mathrm{L}$ of protease inhibitor cocktail (details as seen in Appendix 8) was added to prevent enzymatic protein breakdown during the analysis period. For each analysis time point ( $3 \mathrm{~h}, 24 \mathrm{~h}, 1$ week, 2 week, and 3 week), a $30 \mathrm{uL}$ aliquot was removed from the original sample for tryptic digestion (as described above). The original samples incubated at $37^{\circ} \mathrm{C}$ and were vortexed daily during the analysis period. 


\subsection{Whole Blood Studies}

As a proof of concept study, human whole blood samples (obtained from Bioreclamation, Inc.) were incubated with $\mathrm{HN}-2$ and $\mathrm{HN}-3$ and the target proteins ( $\mathrm{Hb}$ and $\mathrm{HSA}$ ) were isolated and analyzed for adducts identified in Task 3.3. One $\mathrm{mL}$ of whole blood (heparinized) was incubated with HN-2 or HN-3 (dissolved in $40 \mu \mathrm{L}$ HPLC water) at a final concentration of $2 \mathrm{mM}$ for $2 \mathrm{~h}$ under gentle shaking. Following incubation, whole blood samples were centrifuged at $1000 \times g$ for $30 \mathrm{~min}$ at $4^{\circ} \mathrm{C}$ so as to separate the plasma (containing HSA) from the red blood cells (containing $\mathrm{Hb}$ ) and remove leukocytes and platelets.

Serum albumin extraction was performed on separated plasma in two ways - the first was via affinity chromatography extraction, and the second as described in Bechtold et al. ${ }^{127}$ Affinity chromatography extraction of HSA was performed using HiTrap Blue HP affinity columns (1 $\mathrm{mL}$ ) (GE Healthcare, Inc.). Extracted plasma was diluted to twice the original volume using 40 $\mathrm{mM}$ sodium phosphate buffer, $\mathrm{pH} 7.0$ so as to match the composition of the manufacturersuggested binding buffer (20 mM sodium phosphate, $\mathrm{pH}$ 7.0). First, the columns were washed with 10 volumes $(10 \mathrm{~mL})$ of binding buffer using a syringe attached to the top of the column. The diluted plasma sample was then added to the affinity column slowly (approximately $1 \mathrm{~mL} / \mathrm{min}$ ) via syringe. The column was then washed with $10 \mathrm{~mL}$ of binding buffer, and the sample was eluted with $10 \mathrm{~mL}$ of elution buffer ( $20 \mathrm{mM}$ sodium phosphate buffer, $2 \mathrm{M} \mathrm{NaCl}, \mathrm{pH}$ 7.0). The collected eluate was then concentrated to $500 \mu \mathrm{L}$ using Amicon $10 \mathrm{kDa}$ spin filters, followed by buffer exchange to $50 \mathrm{mM}$ AmBic $\mathrm{pH} 8.0$.

For the precipitation protocol of $\mathrm{HSA}$ extraction, an equal volume of $0.5 \mathrm{M} \mathrm{CaCl}_{2}$ was added to the plasma and allowed to incubate at $4^{\circ} \mathrm{C}$ overnight. After incubation, the sample was centrifuged at $900 \times g$ for $20 \mathrm{~min}$ at $4^{\circ} \mathrm{C}$. The supernatant was extracted from the sample, discarding the precipitated fibrinogen. Four volumes of $0.9 \%(\mathrm{w} / \mathrm{v}) \mathrm{NaCl}$ was added to the recovered supernatant to salinate the sample. Nine volumes of acidic alcohol $(0.02 \mathrm{M} \mathrm{HCl}$ in 
$100 \%$ ethanol) was added dropwise to the salinated supernatant and incubated at $37^{\circ} \mathrm{C}$ for $30 \mathrm{~min}$. Samples were then centrifuged at $650 \times g$ for 30 minutes. The supernatant was removed and the precipitate (globin) was discarded. To the recovered supernatant, $0.2 \mathrm{M}$ sodium acetate was added at $1 / 10^{\text {th }}$ the volume of the supernatant, and the solution was allowed to incubate for $15 \mathrm{~min}$ at room temperature. The sample was centrifuged at $650 \times g$ for $5 \mathrm{~min}$. The supernatant was discarded, leaving behind the precipitated HSA. Purified HSA was then washed with $5 \mathrm{~mL}$ of acetone, followed by $5 \mathrm{~mL}$ of diethyl ether, centrifuging at $650 \times \mathrm{g}$ for $5 \mathrm{~min}$ for each wash. The supernatants were discarded and the solid was allowed to dry. Once dry, the solid was weighed and a solution of $10 \mathrm{mg} / \mathrm{mL}$ was created in $50 \mathrm{mM} \mathrm{AmBic}$, $\mathrm{pH} 8.0$. For each sample, $30 \mu \mathrm{L}$ was removed and digested via the protocol above. An additional aliquot was removed to create a 1.0 $\mathrm{mg} / \mathrm{mL}$ sample for direct injection and deconvolution analysis (MS parameters as seen in Appendix 6, deconvolution parameters as seen in Appendix 5).

Hemoglobin precipitation was performed on the isolated red blood cell fraction as reported in DeCaprio et al., ${ }^{128}$ with some modifications. Red blood cells were washed three times with $310 \mathrm{mOsm}$ Tris buffer, $\mathrm{pH} 7.6$ at $1000 \mathrm{x}$ for $30 \mathrm{~min}$ at $4^{\circ} \mathrm{C}$. After the third wash, cells were resuspended in $310 \mathrm{mOsm}$ Tris buffer to $50 \%$ hematocrit. Cells were then lysed by forceful syringe injection of six volumes of $20 \mathrm{mOsm}$ Tris buffer, $\mathrm{pH}$ 6.7. Lysed cells were centrifuged at $20,000 \times g$ for $40 \mathrm{~min}$ at $4^{\circ} \mathrm{C}$. The hemolysate was removed and precipitate was discarded. The hemolysate was then brought to $0.2 \mathrm{M}$ ascorbic acid and was added dropwise to 20 volumes of ice cold acetone and allowed to incubate overnight at $-20^{\circ} \mathrm{C}$. The following day, samples were centrifuged at $1000 \times g$ for $10 \mathrm{~min}$ at $4^{\circ} \mathrm{C}$. The supernatant was discarded and the precipitated $\mathrm{Hb}$ was washed twice with $5 \mathrm{~mL}$ of ice cold acetone by centrifuging at $1000 \times g$ for $10 \mathrm{~min}$ at $4^{\circ} \mathrm{C}$. The precipitated $\mathrm{Hb}$ was allowed to dry. Once dry, the protein was weighed and a $10 \mathrm{mg} / \mathrm{mL}$ solution was created in $50 \mathrm{mM}$ AmBic, $\mathrm{pH}$ 8.0. For each sample, $30 \mu \mathrm{L}$ of sample was removed for tryptic digest (as described above). In addition, a $1.0 \mathrm{mg} / \mathrm{mL}$ sample was also created for 
direct injection and deconvolution analysis (MS parameters as described in Appendix 6, and deconvolution parameters as described in Appendix 5).

To assess the overall success and purity of the described protein extractions, HSA and $\mathrm{Hb}$ were extracted from triplicate whole blood samples not previously incubated with $\mathrm{HN}$ via the extraction methods described above. Proteins were dissolved in $50 \mathrm{mM} \mathrm{AmBic} \mathrm{pH} 8.0$ to create 4 $\mathrm{mg} / \mathrm{mL}$ samples and run via LC-Q-TOF-MS. Chromatographic separation of whole proteins was

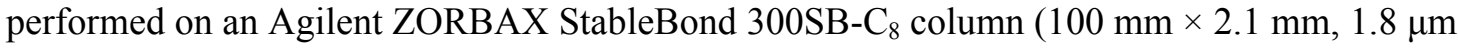
particle size). Extracted protein samples were compared to lyophilized proteins (4 mg/mL solutions in AmBic, $\mathrm{pH}$ 8.0) for a general assessment of purity and for retention time (RT) comparison. In addition, peaks were deconvoluted (method specifications as seen in Appendix 5) and compared to expected molecular weights for $\mathrm{Hb}$ and HSA. Method specifics for $\mathrm{C}_{8}$ separation of whole proteins may be found in Appendix 6.

\subsection{In Vitro Metabolism of Nitrogen Mustard Therapeutics}

For the metabolic analysis of the therapeutic nitrogen mustards Chlorambucil, Cyclophosphamide, and Melphalan, liver homogenate in vitro metabolic systems were utilized to determine whether metabolism products of these three drugs produced the same adducts as their chemical weapon counterparts ( $\mathrm{HN}-2$ and $\mathrm{HN}-3)$. The two biotransformation enzyme systems utilized for this study were human liver microsomes (HLM) and the S9 fraction (S9), as these two fractions are frequently utilized to represent the metabolic activities of the hepatic microenvironment, thus providing a global picture of Phase I liver metabolism of these drugs. The assay components for these in vitro assays are described in Table 4 and were adapted from Schneider and DeCaprio. ${ }^{129}$ 


\begin{tabular}{|c|c|c|}
\hline \multirow{2}{*}{ Component } & \multicolumn{2}{|c|}{ Metabolic System } \\
\cline { 2 - 3 } & HLM & S9 \\
\hline Buffer & $10 \mathrm{mM}$ sodium phosphate buffer, $\mathrm{pH} 7.4$ \\
\hline Substrate & $\begin{array}{c}200 \mu \mathrm{M} \text { positive control (HN-2 or HN-3), } \\
\text { chlorambucil, cyclophosphamide, or melphalan }\end{array}$ \\
\hline Enzyme & $2.0 \mathrm{mg} / \mathrm{mL} \mathrm{HLM}$ & $2.0 \mathrm{mg} / \mathrm{mL} \mathrm{S9}$ \\
\hline Activating Cofactor & \multicolumn{2}{|c|}{$2.0 \mathrm{mM} \mathrm{NADPH}$} \\
\hline Non-activating Cofactor & \multicolumn{2}{|c|}{$3.0 \mathrm{mM} \mathrm{MgCl}$} \\
\hline
\end{tabular}

Table 4: Components of in vitro metabolic system.

All metabolic studies were performed in $10 \mathrm{mM} \mathrm{SPB}$ at $\mathrm{pH} 7.4$ so as to mimic human physiological conditions within the assay. The buffer, substrate, and enzyme system were combined and pre-incubated at $37^{\circ} \mathrm{C}$ for 5 min before adding the assay cofactors $\left(\mathrm{MgCl}_{2}\right.$ and $\mathrm{NADPH}$ ) to the mixture. Samples were allowed to incubate at $37^{\circ} \mathrm{C}$ for $1.5 \mathrm{~h}$. Samples were then quenched with ice cold $6 \%(\mathrm{v} / \mathrm{v})$ acetic acid in acetonitrile and centrifuged at $4{ }^{\circ} \mathrm{C}$ for 30 minutes at $15,000 \times g$ so as to pellet assay proteins. The supernatant was then removed for analysis via LC-QQQ-MS. SIM-MS of expected adduct masses for each trapping peptide (method specifics as seen in Appendix 6) was utilized. The presence or absence of adducts was confirmed by comparing SIM results of the therapeutic mustards to adducts formed with the trapping peptides incubated with $\mathrm{HN}-2$ or $\mathrm{HN}-3$, which are known to form without the need for metabolic activation and should therefore form in the presence of metabolic systems. If no peak was present at the same retention time as compared to the control samples, it was concluded that the drug did not metabolize into a reactive intermediate that forms the same adduct(s) as HN-2 or HN-3.

\subsection{Quantum Mechanical Calculations}

Calculations to determine the chemical potential $(\mu)$, chemical hardness $(\eta)$, and reactivity index $\left(\omega^{-}\right)$were performed first by determining the $\mathrm{E}_{\mathrm{HOMO}}$ and $\mathrm{E}_{\mathrm{LUMO}}$ of the reactive nucleophiles and hypothesized reactive electrophiles from the present study. Energies for each 
compound were determined by optimizing the structure geometry using Chem 3D Ultra software (Version 8, CambridgeSoft Corporation) and exported into Gaussian 03 software (Gaussian, Inc.).

${ }^{130} \mathrm{E}_{\text {номо }}$ and $\mathrm{E}_{\mathrm{LUMO}}$ energies were calculated using a B3LYP function with 6-31G* basis set. Based on these optimized energies, calculations for chemical potential, chemical hardness, and reactivity index were performed based on HSAB Theory as presented in Section 2.3 (Equations $1-3)$. 


\section{RESULTS AND DISCUSSION}

\subsection{Model Peptide Adduct Screening}

\subsubsection{HN-2/HN-3 Adduction to AcPAACAA}

Previous work involving protein adduction to nitrogen mustards such as $\mathrm{HN}-2$ and $\mathrm{HN}-3$

has demonstrated their ability to adduct to $\mathrm{Cys} ;{ }^{41,93,95}$ therefore, $\mathrm{HN}-2$ and $\mathrm{HN}-3$ adduction to the model peptide AcPAACAA was used as a point of reference for adduction to Lys (AcPAAKAA) and His (AcPAAHAA) as alternative sites. Adduction of HN-2 to AcPAACAA was observed via full scan LC-QQQ-MS by the appearance of a peak at $m / z 664$ daltons (Da), corresponding to a mass increase of $119 \mathrm{Da}$, the expected adduction mass increase for HN-2. Furthermore, the isotopic ratio of the adducted product was consistent with that of one chlorine atom present, as would be expected with HN-2 adduction. In addition, MS-MS confirmation of the adduct was performed via Product Ion Scan LC-QQQ-MS. Figure 12 demonstrates the presence of the AcPAACAA adduct peak as compared to a control spectrum, in addition to the isotopic ratio of the product. Further confirmation of HN-2 adduction is demonstrated in the MS-MS spectra for the adduct peak. The MS-MS spectrum shows fragments corresponding to expected peptide fragmentation, in addition to a peak corresponding to the HN-2 aziridinium (Az) ion $(\mathrm{m} / z 120$ Da). When compared to the parent peptide MS-MS, the fragment corresponding to the aziridinium ion is missing. This peak, therefore, is indicative of $\mathrm{HN}-2$ adduction. 

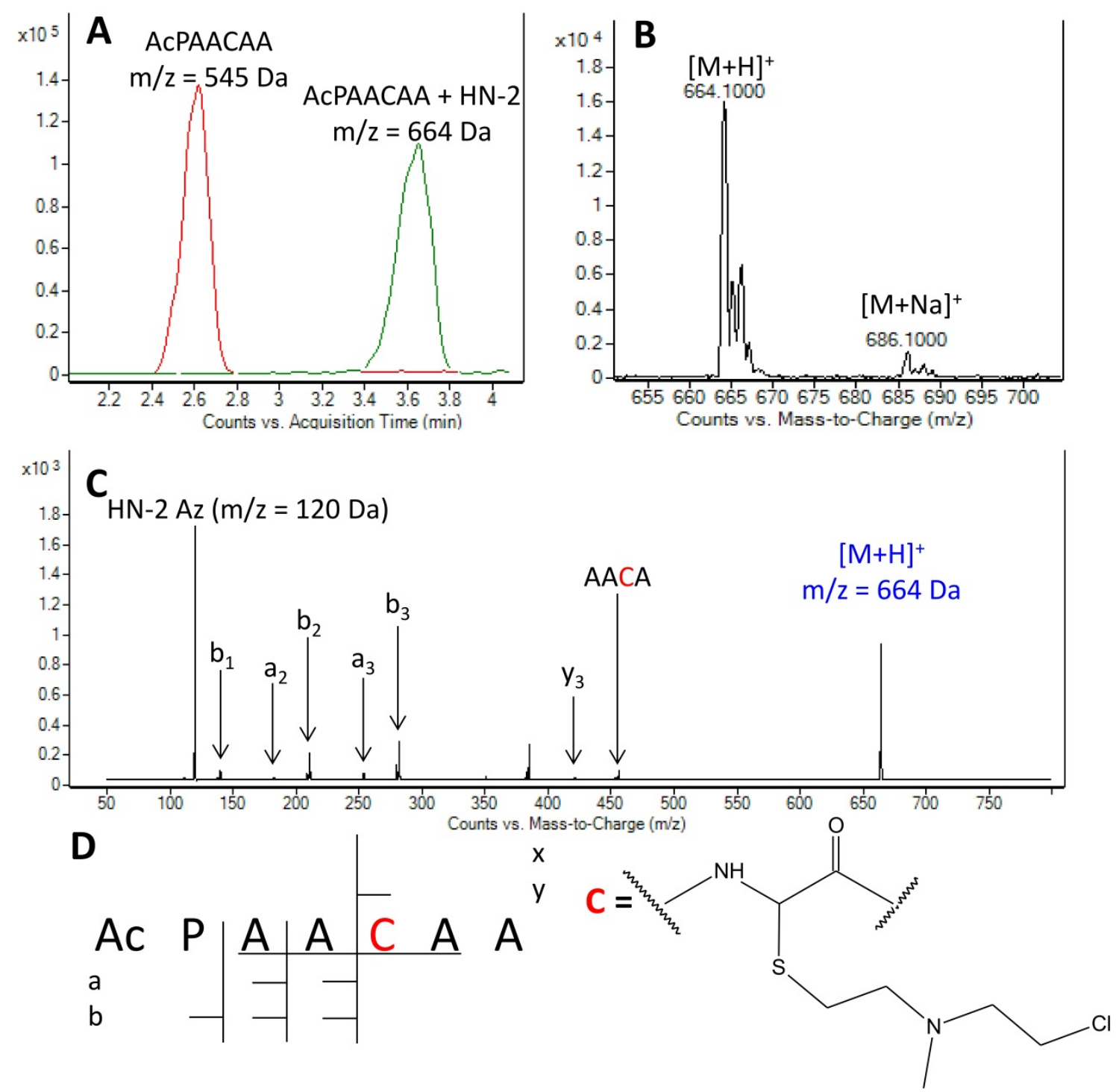

Figure 12: HN-2 adduction to AcPAACAA. (A) EIC of parent [red] and adducted [green] peptide. (B) MS spectrum of adduct peak, demonstrating chlorine isotope. (C) MS-MS spectrum of adduct peak. Precursor ion in seen in blue; adducted Cys in red. (D) Fragmentation diagram of MS-MS data, with adducted Cys residue structure shown.

Adduction of AcPAACAA to HN-3 occurred via a similar manner - incubation of AcPAACAA with excess HN-3 revealed a product peak with a mass increase of $149 \mathrm{Da}$ as compared to the parent peptide. This product is consistent with HN-3 adduction in which one chlorine remained attached to $\mathrm{HN}-3$ and the second chlorine was lost via hydrolysis. This 
conclusion is further supported by the isotopic ratio of the observed product, consistent with one chlorine present within the adduct molecule, as can be seen in Figure 13.
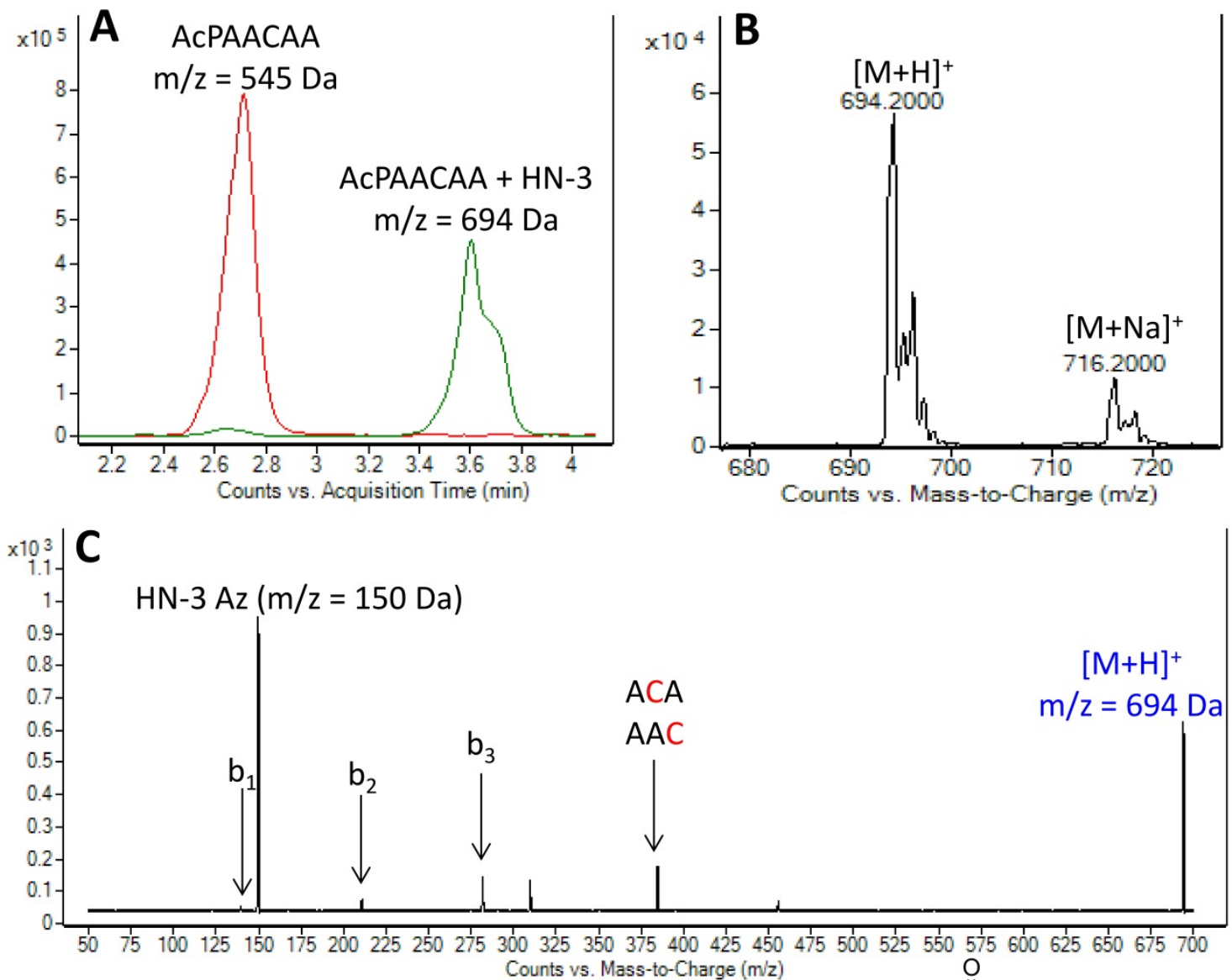

D

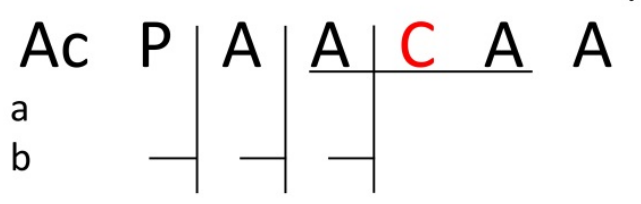

$\mathrm{X}$

$y$<smiles>C=C[C@H](C)NC(SCCN(CCO)CCCl)C(=C)CC</smiles>

Figure 13: HN-3 adduction of AcPAACAA. (A) EIC of parent [red] and adducted [green] peptide. (B) MS spectrum of adduct peak, isotope ratio demonstrates one chlorine attached. (C) MS-MS spectrum of adduct. Precursor ion highlighted in blue, adducted Cys in red. (D) Fragmentation diagram of MS-MS data, with adducted Cys structure shown.

The exposure-response relationship of $\mathrm{HN}-2$ and $\mathrm{HN}-3$ incubation to AcPAACAA was also determined as a reference for future studies. Full scan analysis demonstrated that the lowest 
molar ratios for observable adduction were 100:1 molar excess for HN-2 and 50:1 molar excess for HN-3. It is important to note that, throughout these adduction studies, complete adduction of the model peptides was never observed, nor was it expected to occur based on literature reports. ${ }^{131}$ Based on this data, a 100:1 ratio of nitrogen mustard:peptide ratio was selected for AcPAACAA adduction in further experiments.

\subsubsection{HN-2/HN-3 Adduction to AcPAAKAA}

Adduction of HN-2 to the Lys residue of AcPAAKAA was observed by the appearance of a peak with $\mathrm{m} / \mathrm{z} 711 \mathrm{Da}$ following HN-2 incubation. This corresponds to a mass increase from the parent peptide of $141 \mathrm{Da}$, or an $\mathrm{HN}-2$ adduct (119 Da) with a sodium ion (+22 Da). Electrospray ionization is known to produce sodium adducts in sodium phosphate buffer, which may be more abundant than traditional $\mathrm{M}+\mathrm{H}$ ions in certain cases. This assignment can be further confirmed by analysis of the MS spectrum (Figure 14) of the adduct peak more closely. The appearance of the HN-2 adduct without the sodium $(\mathrm{m} / z 689 \mathrm{Da})$ is present, albeit at a lower intensity. In addition, the isotopic ratios demonstrate the presence of one chlorine attached to the product molecule, similar to what was seen with Cys adduction. 

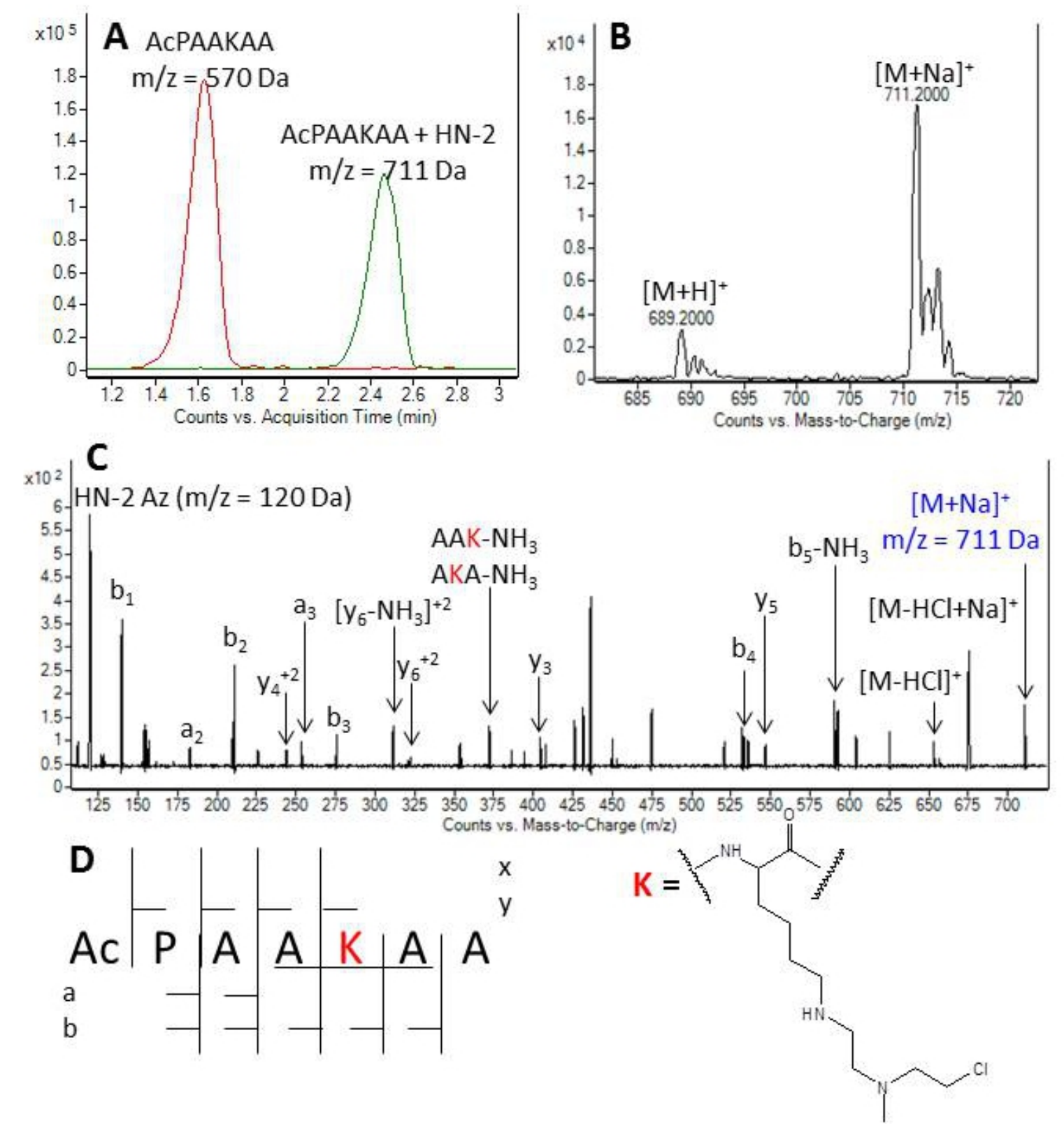

Figure 14: HN-2 adduction to AcPAAKAA. (A) EIC of the parent [red] and adducted [green] peptide; (B) MS spectrum of adduct peaks, with isotopic ratios consistent with one chlorine attached; (C) Labeled MS-MS spectrum of the Lys-HN-2 adduct (precursor ion in blue, adducted Lys in red). (D) Fragmentation diagram of MS-MS data, with adducted Lys structure shown.

Adduction of HN-3 to AcPAAKAA occurred in a similar fashion. After HN-3 incubation, a peak corresponding to $\mathrm{m} / \mathrm{z} 741$ was observed, corresponding to a mass increase of $171 \mathrm{Da}$ from the parent peptide. This mass increase is consistent with a sodium adduct ( $+22 \mathrm{Da}$ ) of the HN-3 
adduction product (149 Da), similar to what was observed for Cys adduction. This can be further confirmed via the MS spectrum of the product, whose isotopic ratio is consistent with one chlorine present in the molecule (Figure 15).

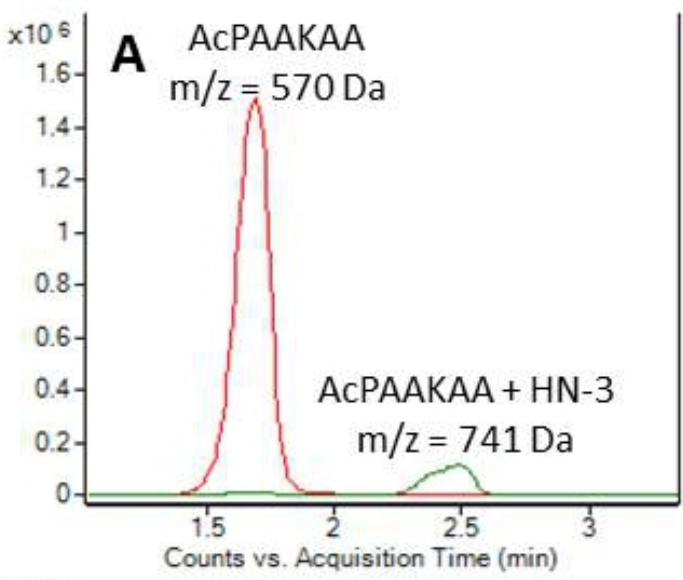

$\times 10^{2}$
2.75

2.5

2.25

1.75

1.75
1.5

1.25

0.75

0.5

0.25

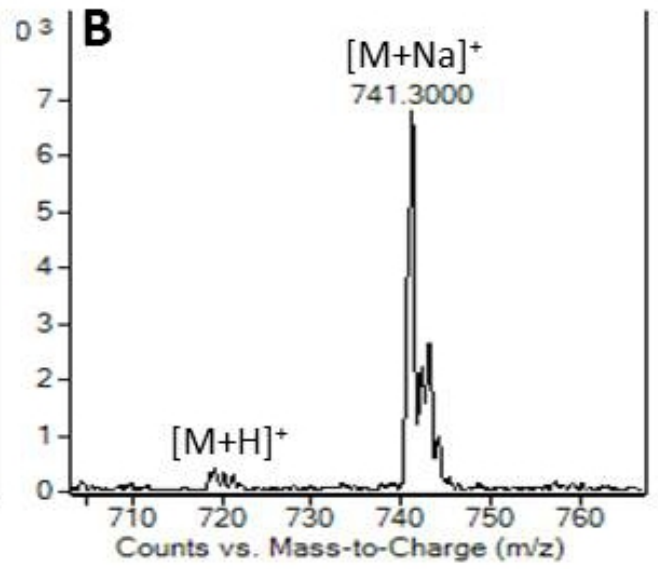

Counts vs. Mass-to-Charge $(\mathrm{m} / \mathrm{z})$

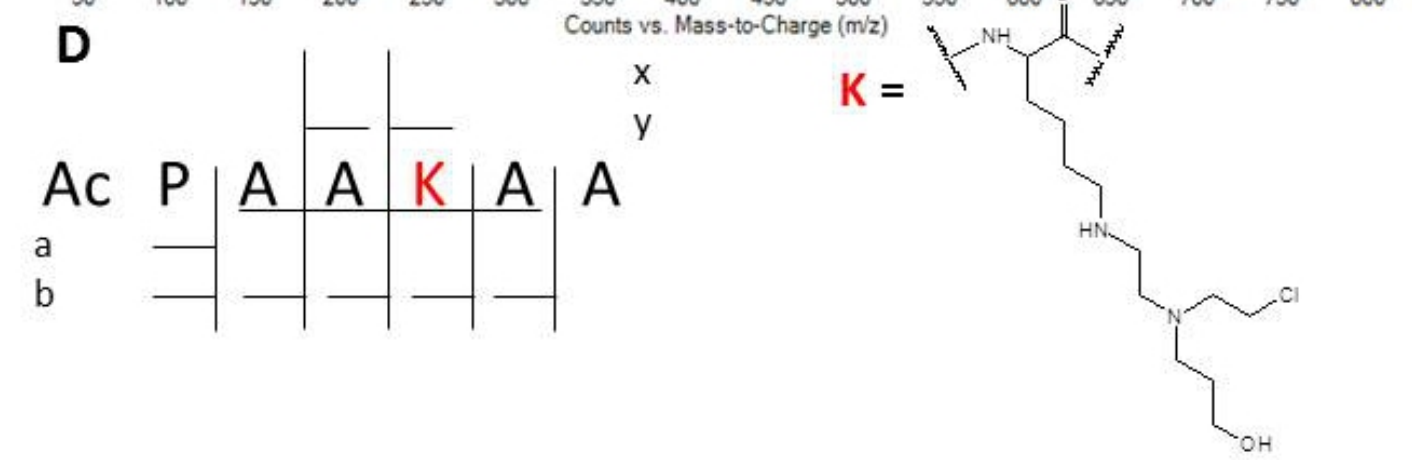

Figure 15: HN-3 adduction to AcPAAKAA. (A) EIC of parent [red] and adducted [green] peptide. (B) MS spectrum of adduct peak, demonstrating isotopic ratios consistent with one chlorine present.

(C) Labeled MS-MS spectrum. Precursor ion in blue, adducted Lys in red. (D) Fragmentation diagram based on MS-MS data, with adducted Lys residue structure shown. 
The exposure-response relationship of adduction to AcPAAKAA was determined for HN-2 and HN-3. In both cases, the lowest observed excess ratio of nitrogen mustard was 100:1, which was consistent to what was seen with AcPAACAA adduction. For this reason, the 100:1 molar excess of $\mathrm{HN}$ was utilized for Lys adduction in further studies.

\subsubsection{HN-2/HN-3 Adduction to AcPAAHAA}

HN-2 adduction to AcPAAHAA was determined by the appearance of an adduct peak with $m / z 720 \mathrm{Da}$, corresponding to a mass increase of $141 \mathrm{Da}$. As with AcPAAKAA adduction, sodium adducts (+22 Da) of the HN-2 adduct (mass increase of $119 \mathrm{Da}$ ) were observed, as can be seen in the analysis of the MS spectra in Figure 16. As seen previously, isotopic ratios of the adduct peak are consistent with one chlorine attached to the product.

A similar adduction pattern was seen with HN-3 (Figure 17). Following incubation of HN-3 with AcPAAHAA, an adduct peak of $m / z 750$ Da was observed, a mass increase of $171 \mathrm{Da}$ from the parent peptide. This corresponds to HN-3 adduction (149 Da) of the peptide with a sodium adduct (+22 Da). As with previously identified adducts, the isotopic ratio of the adduct peak is consistent with one chlorine attached to the molecule.

Finally, the exposure-response relationship of adduction to AcPAAHAA was determined. For both HN-2 and HN-3, the lowest observed molar excess of HN was 100:1 nitrogen mustard:peptide, demonstrating consistent results as seen with both AcPAACAA and AcPAAKAA. As with the other two peptides, 100:1 molar excess of $\mathrm{HN}$ was selected as the ratio for His adduction for further studies. 

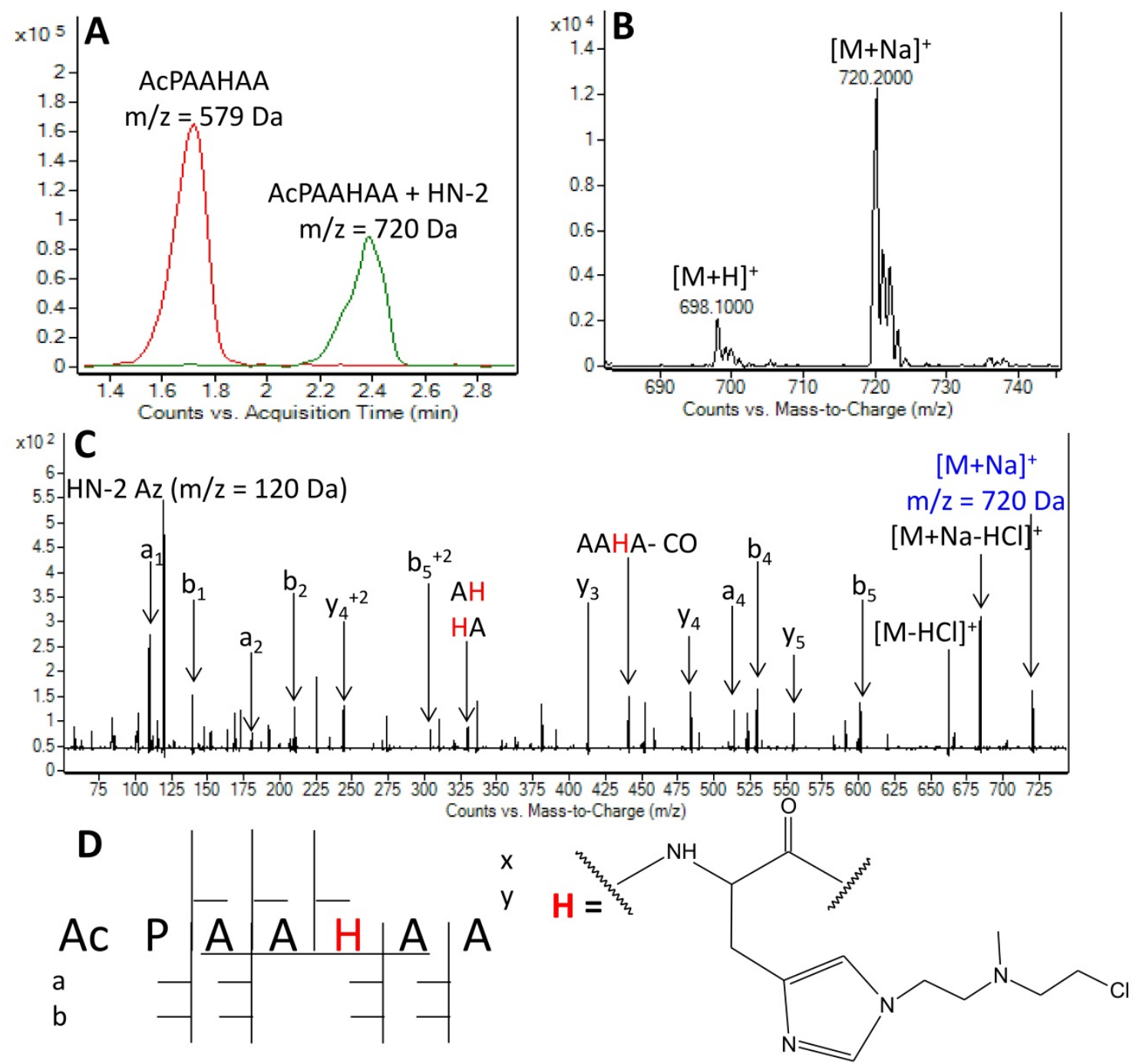

Figure 16: HN-2 adduction of AcPAAHAA. (A) EIC of parent [red] and adducted [green] peptide.

(B) MS spectrum of adduct, demonstrating chlorine isotope. (C) MS-MS spectrum of adduct.

Precursor ion in blue, adducted His in red. (D) Fragmentation diagram based on MS-MS data, with adducted His residue structure shown. 

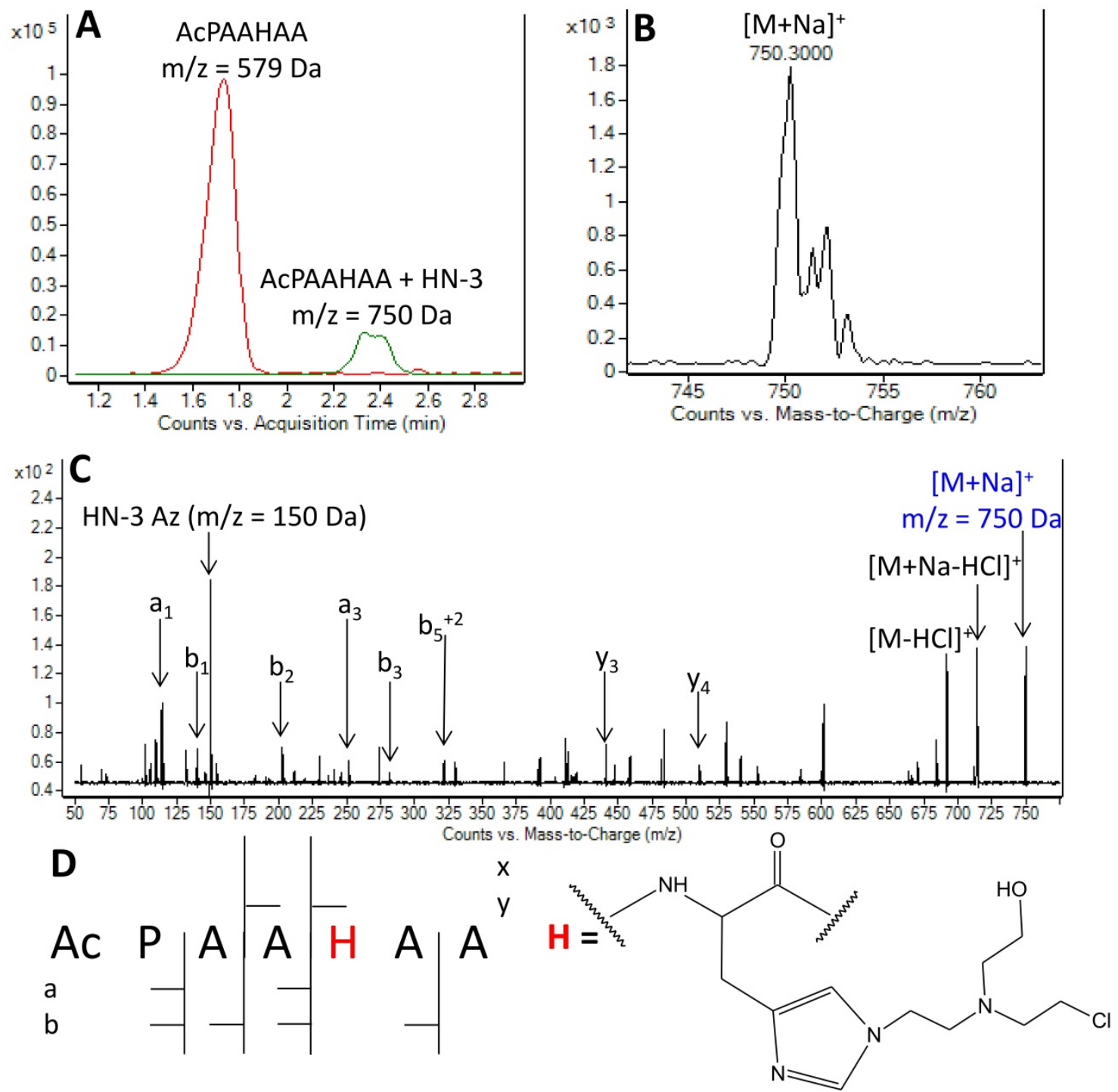

Figure 17: HN-3 adduction of AcPAAHAA. (A) EIC of parent [red] and adducted [green] peptide. (B) MS spectrum of adduct, demonstrating chlorine isotope. (C) MS-MS spectrum of adduct. Precursor ion in blue, adducted His in red. (D) Fragmentation diagram based on MS-MS data, with adducted His residue structure shown.

\subsubsection{Specificity of Identified Adducts based on Precursor Incubations}

All three model peptides were incubated with $\mathrm{HN}-2$ and $\mathrm{HN}-3$ precursors $\mathrm{N}-$

methyldiethanolamine and triethanolamine (Figure 18) to assess whether adducts would form with these compounds. These ethanolamine compounds are not only the synthetic precursors to $\mathrm{HN}-2$ and HN-3, but they are also the hydrolysis products of these compounds that may be found 
in biological specimens. This task was therefore important to assess the usefulness of these adducts as potential exposure biomarkers - should the same adducts form with these precursor molecules, these adducts would not be specific to $\mathrm{HN}-2$ or $\mathrm{HN}-3$ exposure.

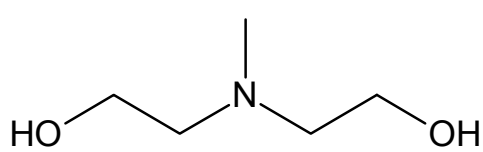

$\mathrm{N}$-methyldiethanolamine

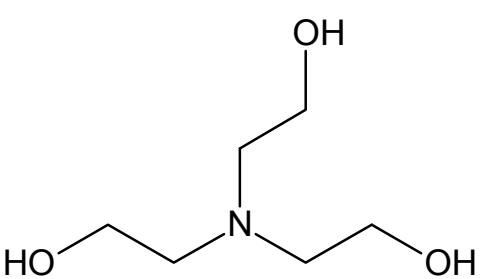

triethanolamine

Figure 18: Structure of the HN-2 and HN-3 precursors N-methyldiethanolamine (HN-2) and triethanolamine $(\mathrm{HN}-3)$.

Incubation of the ethanolamine compounds with the model peptides for $24 \mathrm{~h}$ did not produce any adducts on the model peptides; as such, identified adducts are specific to $\mathrm{HN}-2$ and HN-3 exposure when compared to their ethanolamine precursors.

\subsubsection{Formation Kinetics of Model Peptide Adducts}

The kinetics of adduction for both HN-2 and HN-3 to AcPAACAA produced similar results. Adducts to $\mathrm{HN}-2$ and $\mathrm{HN}-3$ were observed within 30 min of incubation with AcPAACAA. For HN-2, the amount of adduct increased steadily until approximately $4 \mathrm{~h}$, after which a steady-state of adduct was obtained. In contrast, HN-3 adduction reached a plateau within about $2 \mathrm{~h}$, after which a slight decrease in adduct was observed. This was most likely a result of the hydrolysis of the $\mathrm{HN}-3$ adduct (loss of $-\mathrm{Cl}$ to $-\mathrm{OH}$ ), which occurs in aqueous environments. ${ }^{132}$ This hydrolysis would decrease the detected amount of chlorinated adduct and thus lower the ratio observed. Figure 19 demonstrates the kinetics of formation for $\mathrm{HN}-2$ and $\mathrm{HN}-$ 3 over an $8 \mathrm{~h}$ analysis period. 


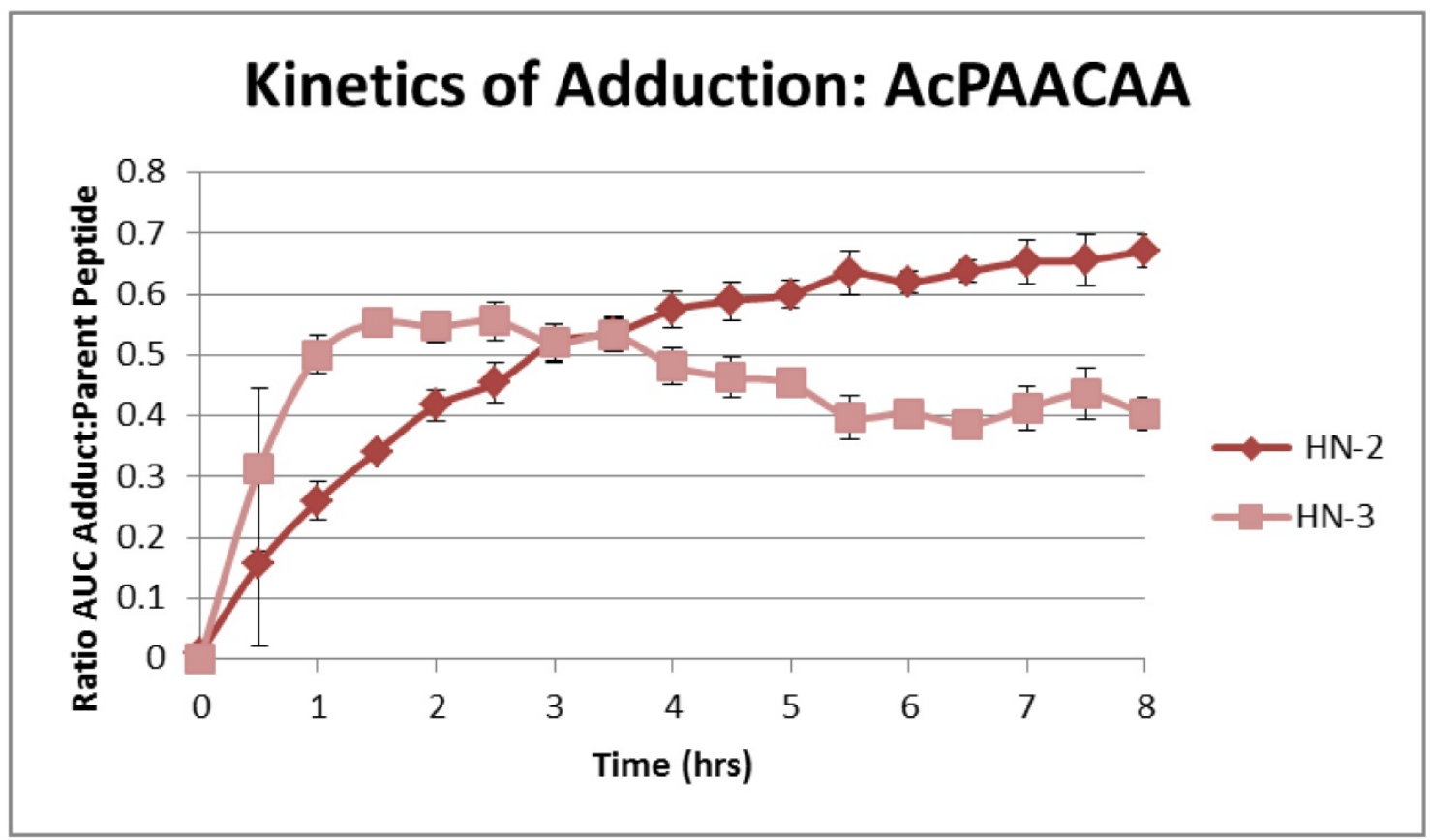

Figure 19: Relative kinetics of formation of HN-2 [red] and HN-3 [pink] adducts to AcPAACAA. Error bars represent standard deviation obtained from triplicate samples.

HN-2 adducts with AcPAAKAA were present within 30 min of incubation, and demonstrated a steady increase until approximately $3 \mathrm{~h}$, after which a steady-state of adduction occurred. In contrast, adducts of HN-3 were not observed until $1 \mathrm{~h}$ after incubation. However, as with $\mathrm{HN}-2$, adduction demonstrated a steady increase until approximately 3-3.5 h, after which a steady state was achieved (Figure 20). 


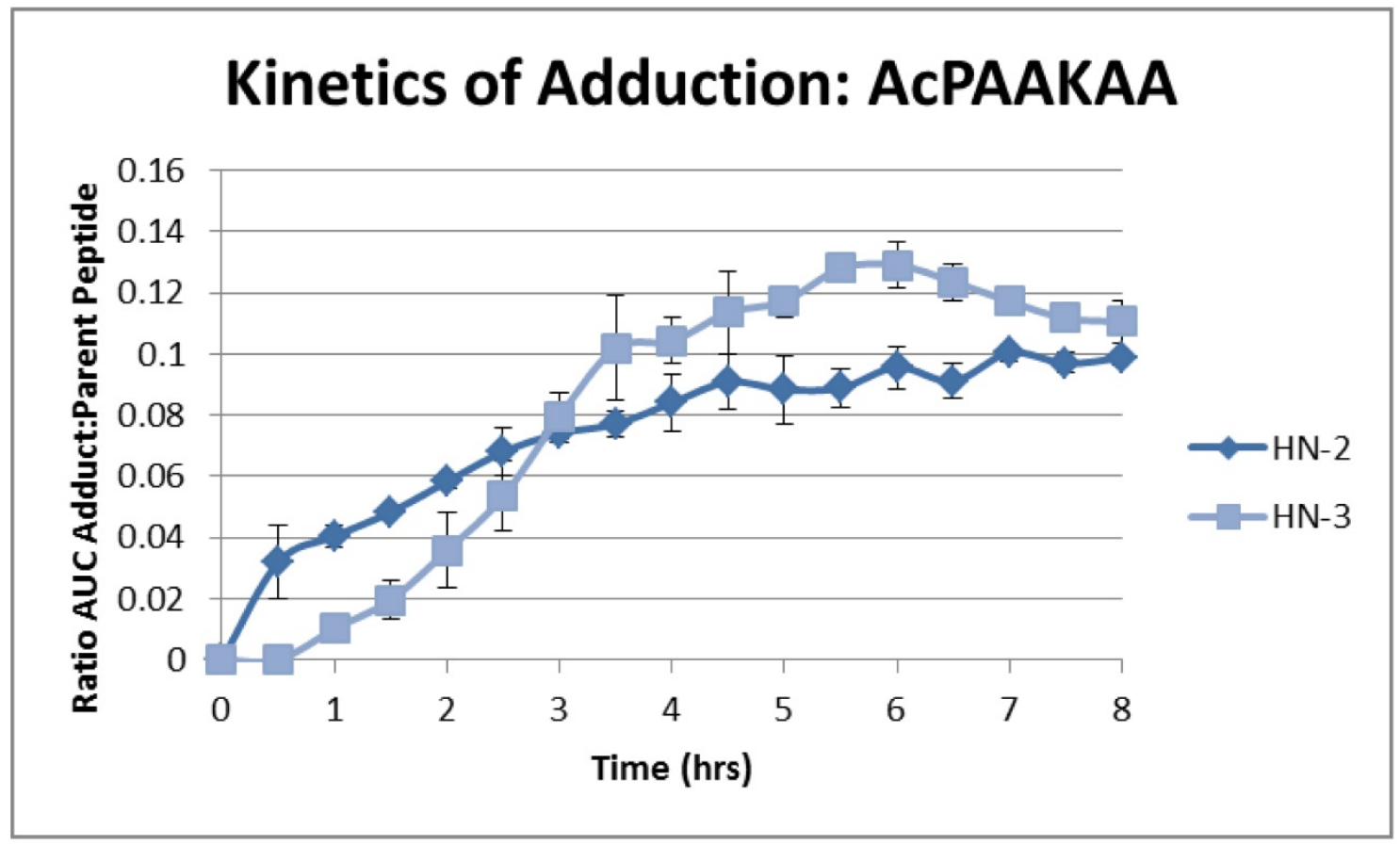

Figure 20: Relative kinetics of formation of $\mathrm{HN}-2$ [dark blue] and HN-3 [light blue] adducts to AcPAAKAA. Error bars represent standard deviation obtained from triplicate samples.

The kinetics of adduction of $\mathrm{HN}-2$ and $\mathrm{HN}-3$ for AcPAAHAA followed a similar pattern to what was seen with AcPAAKAA (Figure 21). For HN-2, adduction was observed after 30 min of incubation with $\mathrm{HN}-2$, and reached a steady state of adduction at approximately $3 \mathrm{~h}$. Adduction to $\mathrm{HN}-3$ was not observed until $1 \mathrm{~h}$ after incubation, with a steady-state reached after $4 \mathrm{~h}$. 


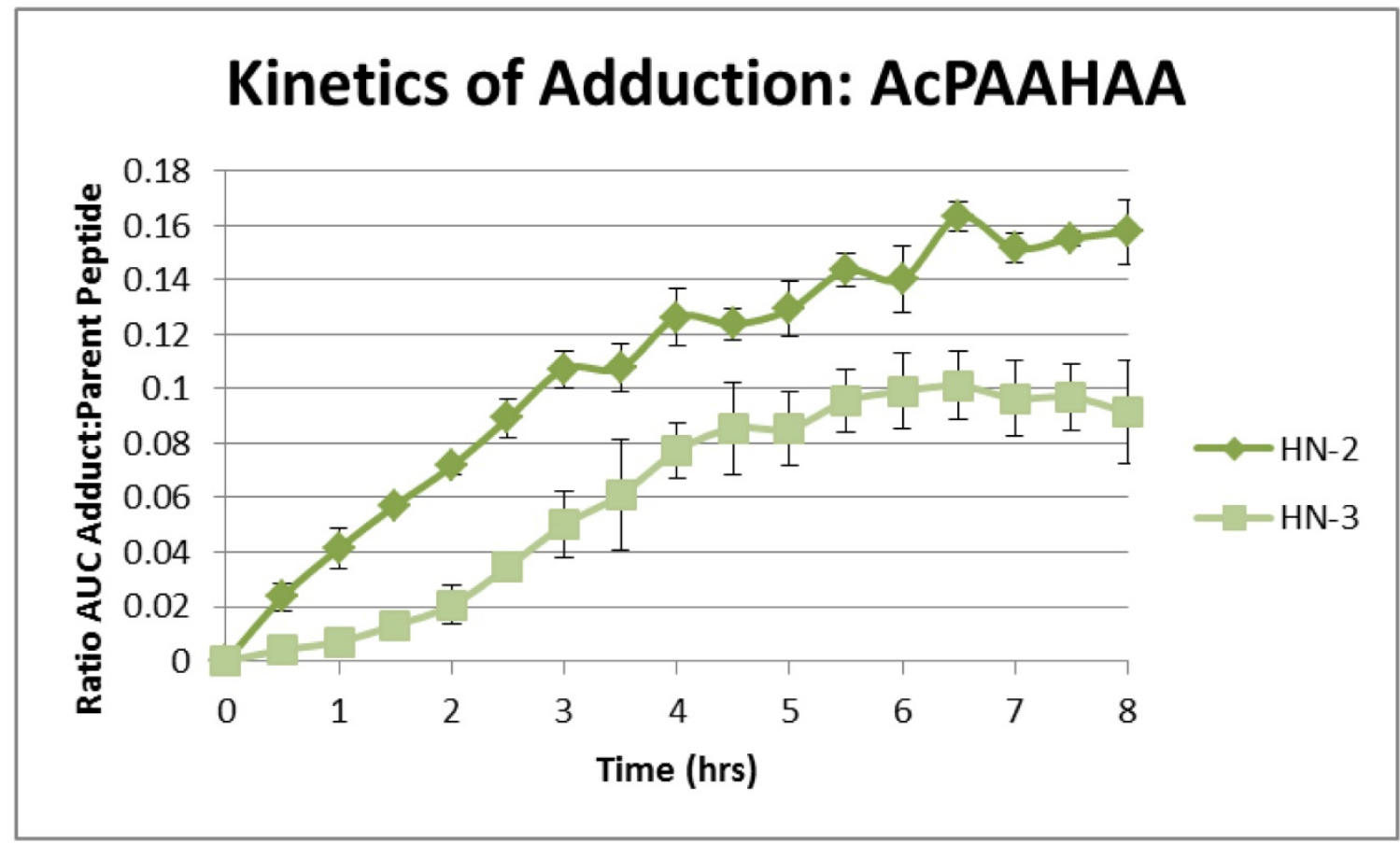

Figure 21: Relative kinetics of formation of $\mathrm{HN}-2$ [dark green] and $\mathrm{HN}-3$ [light green] adducts to AcPAАНАA. Error bars represent standard deviation obtained from triplicate samples.

As mentioned previously, adduction of $\mathrm{HN}-2$ and $\mathrm{HN}-3$ to AcPAACAA was utilized as a comparison point for adduction to AcPAAKAA and AcPAAHAA. While adduction of HN-2 and HN-3 was observed on all three model peptides, a definite contrast can be seen when comparing the relative kinetics of adduction to Cys as compared to Lys and His. As can be seen in Figure 22, the rate of adduct formation of $\mathrm{HN}-2$ and $\mathrm{HN}-3$ with AcPAACAA (red) is much higher than AcPAAKAA (blue) and AcPAAHAA (green). This is to be expected, as Cys is considered a "softer" nucleophile and is therefore likely to be more reactive towards electrophilic adduction than the "moderately hard" Lys and His. However, as nitrogen mustards (specifically, chemotherapeutic agents containing the nitrogen mustard group) have been documented to adduct to nitrogen centers, ${ }^{105}$ it was expected that adduction of $\mathrm{HN}-2$ and $\mathrm{HN}-3$ to nitrogen-containing side chains on Lys and His residues would also occur. Also noted in Figure 22 is a reversal in the kinetics of His and Lys between HN-2 and HN-3 adduction - for HN-2, His adduction is more 
prominent, whereas the opposite is true for HN-3. This may be due to the steric bulk of the HN-3 Az species adducting to the His imidazole, which is not as easily accessible as the Lys amine group. Finally, the slight lag apparent in the adduction of HN-3 to Lys and His (as compared to $\mathrm{HN}-2$ ) is most likely due to the increased reactivity of $\mathrm{HN}-2$ over $\mathrm{HN}-3$, since the hypothesized reactive species for $\mathrm{HN}-3$ (HN-3 Az with one hydrolyzed $\mathrm{Cl}$ ) may take longer to form than the reactive $\mathrm{HN}-2$ species.
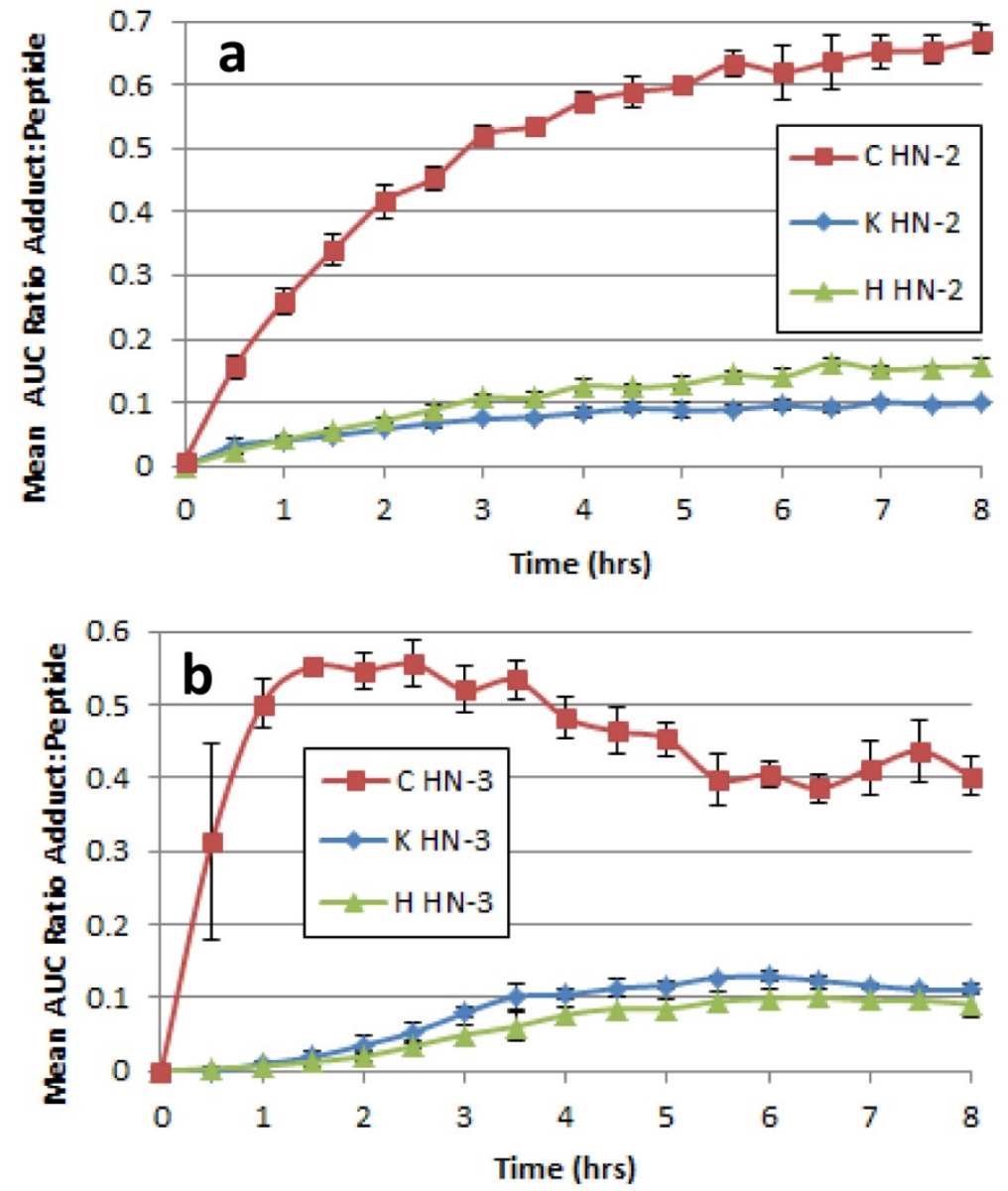

Figure 22: Comparison of formation kinetics for HN-2 (a) and HN-3 (b) for AcPAACAA [red], AcPAAKAA [blue], and AcPAAHAA [green].

With the exception of the relative kinetics of formation, the results obtained from the adduction of HN-2 and HN-3 to all three model peptides were consistent. A general steady state of adduction was achieved for all six adduct products at approximately $3 \mathrm{~h}$; therefore, for later 
experiments, a minimum of $3 \mathrm{~h}$ was utilized for adduction. In addition, the lowest observed ratio of excess HN was 100:1 in five of six instances; therefore, later work involving peptides utilized this molar excess ratio of HN:peptide. The consistency observed for the model peptide studies suggest that, when scaled up to full proteins, $\mathrm{HN}-2$ and $\mathrm{HN}-3$ adduction would be expected to occur on Lys and His residues in addition to the previously demonstrated Cys adduction.

\subsubsection{Stability of Model Peptide Adducts}

The stability of HN-2 and HN-3 adducts was observed over a three-week incubation period. Throughout this period, samples were kept at physiological conditions $\left(\mathrm{pH} 7.4,37^{\circ} \mathrm{C}\right)$ to mimic the stability of these adducts in the body. During the analysis process, the products described above (herein described as "-Cl adducts," as seen in Figure 23) were monitored via full scan MS. In addition, products corresponding to hydrolysis of all chlorine atoms present on the adduct (herein described as "-OH adducts") were also monitored. As these adducts were present in aqueous environment for an extended period of time, previous work regarding nitrogen mustard adduction ${ }^{133}$ suggested that $-\mathrm{OH}$ adducts may be more prominent than the previously identified $-\mathrm{Cl}$ adducts. All $-\mathrm{OH}$ adducts were confirmed via MS-MS analysis and RT matching from previous analysis (Appendix 6). 


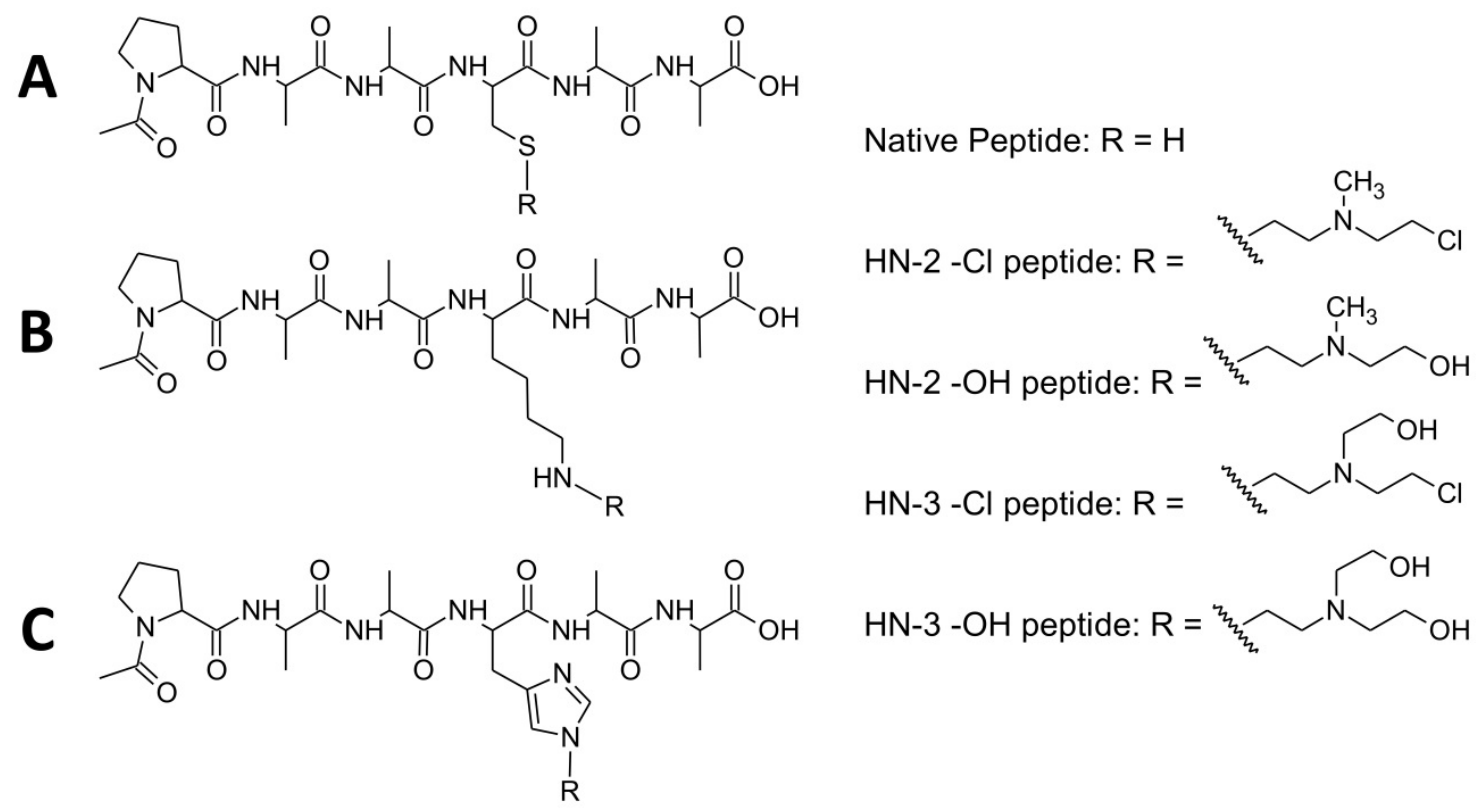

Figure 23: Structures of identified peptide adducts; $(A)=$ Cys; $(B)=$ Lys; $(C)=$ His.

For $\mathrm{HN}-2$, the $-\mathrm{Cl}$ adducts for all three model peptides were detected throughout the three-week analysis period. In addition,- $-\mathrm{OH}$ adducts were observed starting $24 \mathrm{~h}$ after initial $\mathrm{HN}$ 2 incubation. All three $-\mathrm{Cl}$ adducts exhibited a decrease in signal as the analysis period progressed, paired with a corresponding increase of $-\mathrm{OH}$ adduct over time. As mentioned previously, this was expected based on previously published results, and may suggest that the $\mathrm{OH}$ adducts may be more relevant for full protein and long term exposure biomarker studies.

For $\mathrm{HN}-3$, the $-\mathrm{Cl}$ adducts were present for all three products for the first two time points ( $3 \mathrm{~h}$ and $24 \mathrm{~h}$ ); however, they were not detected at the one week time point for AcPAACAA and AcPAAHAA. Chlorinated adducts of AcPAAKAA were present throughout the three-week analysis period, but at substantially lower abundance than the observed $-\mathrm{OH}$ adducts. Despite the fact that the $-\mathrm{Cl}$ adducts were not present after $24 \mathrm{~h}$ for AcPAACAA and AcPAAHAA, the -OH adducts were observed during the three week analysis period. An HN-3 molecule containing two bulky side chains is more likely to experience steric hindrance; therefore, it may hydrolyze more rapidly as compared to the less bulky $\mathrm{HN}-2$ adduct. The $-\mathrm{Cl}$ adduct attached to AcPAAKAA may 
persist longer due to the decreased steric stress of adduction on the long side chain of Lys as compared to the smaller, more hindered chains of Cys and His. Regardless, these results also suggest that the fully hydroxylated adducts may be more relevant to monitor for full protein studies, in which steric factors will play a much larger role than they do in model peptides. Results for the stability of both HN-2 and HN-3 adducts can be seen in Figure 24 . 

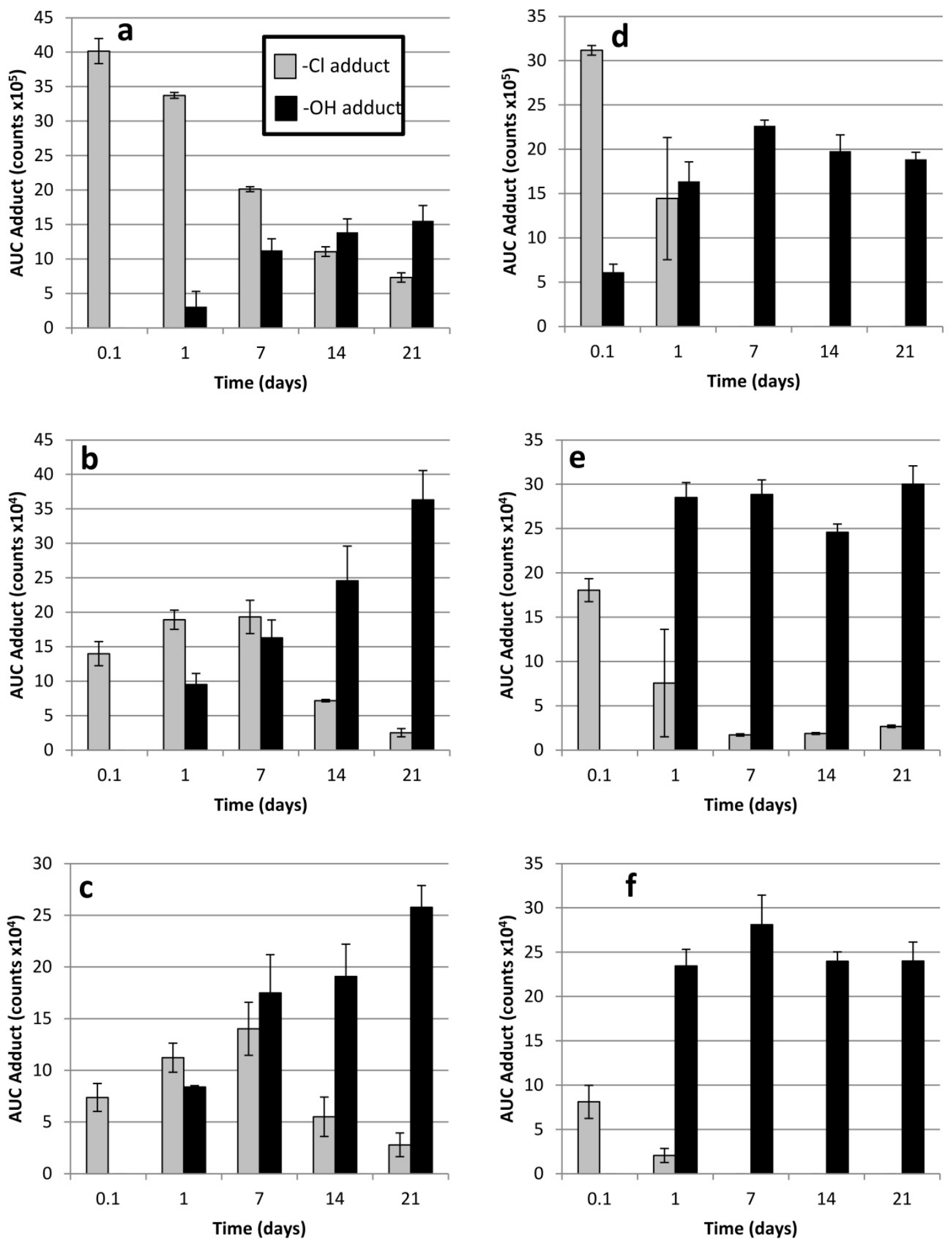

Figure 24: Stability of HN-2 (a-c) and HN-3 (d-f) over three weeks. (a, d): Adduction of AcPAACAA. $(b, e)$ : Adduction of AcPAAKAA. (c, f): Adduction of AcPAAHAA. 


\subsubsection{Discussion}

Results of characterization of HN-2 and HN-3 adduction to Lys and His residues in the model peptides AcPAAKAA and AcPAAHAA were compared to results of adduction at a known reactive moiety (Cys; AcPAACAA). The results from the present work confirm previous reports demonstrating that HN-2 and HN-3 will adduct with Cys residues. More importantly, however, this work also demonstrates that adduction of these nitrogen mustards can also occur at Lys and His, a finding that was fully confirmed via isotopic ratios and MS-MS results. While adduction may occur more readily at Cys residues in the model peptide system employed here, adducts to Lys and His were observed to be equally stable as Cys adducts over a three week analysis period. This was particularly true for the fully hydroxylated adducts, which is the focus of full protein adduction studies described later.

It is important to note that the results obtained here are representative of a model system in which several factors (most notably steric) have been carefully controlled so as to assess adduction potential at the nucleophilic sites of interest (Cys, Lys, and His) in the absence of higher order influences. It is recognized that the site-specific kinetics of formation and interactions between nucleophilic sites and the electrophilic $\mathrm{HN}$ intermediates observed in this task, while important, may not be identical to those obtained for full proteins. This prediction is based on the previously described mediators of adduction in proteins, including steric factors in addition to electronic microenvironments surrounding a potential adduction site (discussed in section 2.3). As such, the observation that Lys and His adduction were comparable with Cys in model systems would not necessarily guarantee that the same results will be obtained in full proteins.

For initial adduct determination and molar ratio studies, Full Scan MS mode was selected instead of other, likely more sensitive methods (such as SIM or MRM). Full Scan MS was determined to be more applicable to the planned later experiments in which full proteins would be 
incubated with $\mathrm{HN}-2$ and $\mathrm{HN}-3$. For these reasons, a higher molar excess than would be likely to occur in vivo was selected to confirm adduction. It is believed that a lower ratio could have been utilized with the more sensitive MS approaches listed above; however, as the purpose of this study was primarily to demonstrate that adduction could occur with lysine and histidine in principle, it was deemed appropriate for this task. 


\subsection{Full Protein Adduction Studies}

The ultimate goal of this work was to identify novel, alternative protein adduct based biomarkers of exposure of HN-2 and HN-3, specifically on Lys and His residues. As these adducts have not been previously documented, it was of extreme importance that a high level of discrimination be used before a suspected adduct could be positively identified. Therefore, a series of criteria was implemented so as to maximize confidence in the results presented. While these strict criteria may mean that other adducts could have been falsely eliminated, they lend a high level of specificity to the results presented herein.

The software utilized for the identification of adducts was Agilent BioConfirm v. 5.0 (Specific parameters utilized for analysis may be found in Appendix 5). With this software, users can input post-translational modifications (whether standard within the program or in-house additions) to a matching algorithm, which will take a given protein sequence and the userspecified modifications and selectively match the data obtained from the instrument to possible peptides. The software provides a large amount of information, including a mass difference from the theoretical fragment and the location of any modifications identified within the peptide. While this matching algorithm is useful, at times the software can make assignments that do not follow certain rules. For example, a modification may be placed on the terminal Lys of a tryptic peptide, which would not be possible since trypsin would not be able to cleave at that location if the Lys were modified as a result of steric interference from the adduct moiety. The software may also place several modifications on adjacent residues, which may not be possible due to the potential steric strain on the molecule. These examples are a consequence of the "force fit" mechanism that the software employs.

For these reasons, it was important to follow up any software-identified adduct with independent MS-MS data support. For the full protein work, the MS-MS fragmentation for a potential adducted peptide was confirmed manually using theoretical fragmentation data obtained 
from Protein Prospector, ${ }^{126}$ in which additional fragments besides $b$ and $y$ could be utilized for higher confidence identification. BioConfirm is programmed to label $b$ and $y$ fragments; however, it will at times miss some (i.e., may not label a fragment as $b$ or $y$ despite its concordance with Protein Prospector's theoretical peak list), and does not consider internal fragmentation or $a$ fragments, which are also commonly encountered with low-energy CID. ${ }^{134}$ Consequently, MSMS data were also analyzed by manual techniques to confirm a true positive identification.

Finally, it was also important to ensure that an identified adduct was reproducible in multiple replicate samples. The Q-TOF MS-MS method employed Auto MS-MS, meaning that (based on manufacturer-recommended settings) the three most abundant ions in each scan were selected to trigger MS-MS analysis. However, because of the variability of individual sample digests, a particular ion may have triggered MS-MS in one sample, but not in another. For this reason, it was important to set limits of acceptable variation when looking at multiple samples, balanced as much as possible with a low false positive rate.

When all of these factors were taken into consideration, the following rules were followed in order to ensure that a high level of confidence was present in the identification of adducts on intact model proteins:

- For each experimental run, triplicate samples of each condition (HN-2 or HN-3) were analyzed for reproducibility.

- A control protein sample (incubated in the absence of $\mathrm{HN}-2$ or $\mathrm{HN}-3$ ) was prepared and utilized to eliminate false positives identified by the software. False positives were defined as any peptide in the control sample that the software errantly determined to contain a HN modification. False positives were located within the software generated compound (ion) list for the HN-2 and HN-3-incubated samples, and if the description (same peptide and adduction site) and retention time (RT) matched with a previously designated false positive, they were eliminated from further analysis. 
- In each sample, any compound that was determined by the software to have an HN modification and MS-MS support was analyzed by hand. Theoretical peak lists for the MS-MS spectra were obtained from Protein Prospector (specific search parameters are as seen in Appendix 7). An MS-MS fragment was considered positively identified if peak mass matching between empirical data and theoretical Protein Prospector data was concordant within one-tenth of a Dalton. If a fragment mass deviated by more than onetenth of a Dalton from the theoretical mass, it remained in the pool of candidate peptides if a positively identified second ion was present for support (For example, if the $\mathrm{y}_{5}$ ion deviated from theory by more than one-tenth of a Dalton, it would only be labeled if a supporting ion, such as $\mathrm{y}_{5}^{+2}$ could be positively identified within the MS-MS spectra).

- Within each experiment, the triplicates were compared for consistency of identified adducts. In each sample, an adduct could be either 1) present in the peak list with confirmed MS-MS data, 2) present in the peak list, but without MS-MS data, 3) present in the peak list, but at a different RT, or 4) not present. Table 5 below demonstrates various scenarios that could be obtained from triplicate samples, and what requirements were necessary for a positive identification in each case. 


\begin{tabular}{|c|c|c|c|}
\hline \multicolumn{2}{|c|}{ Condition } & Positive \\
\hline Sample 1 (S1) & Sample 2 (S2) & Sample 3 (S3) & Yes \\
\hline MS-MS confirmation & MS-MS confirmation & MS-MS confirmation & Yes \\
\hline MS-MS confirmation & MS-MS confirmation & $\begin{array}{c}\text { Cpnd at same RT as S1 } \\
\text { and S2; no MS-MS } \\
\text { collected by software }\end{array}$ & No \\
\hline $\begin{array}{c}\text { MS-MS confirmation } \\
\text { MS collected by } \\
\text { software }\end{array}$ & $\begin{array}{c}\text { Cpnd at same RT as S1; } \\
\text { no MS-MS collected by } \\
\text { software }\end{array}$ & $\begin{array}{c}\text { Cpnd at same RT as S1; } \\
\text { no MS-MS collected by } \\
\text { software }\end{array}$ & No MS-MS collected by \\
MS-MS confirmation & $\begin{array}{c}\text { Cpnd at same RT as S1; } \\
\text { no MS-MS collected by } \\
\text { software }\end{array}$ & No \\
\hline MS-MS confirmation & $\begin{array}{c}\text { Cpnd at same RT as S1; } \\
\text { no MS-MS collected by } \\
\text { software }\end{array}$ & Not present in peak list & No \\
\hline MS-MS confirmation & $\begin{array}{c}\text { Present, but at RT }> \pm \\
\text { 0.3 min from S1; MS- } \\
\text { MS confirm? }\end{array}$ & Not present in peak list & No \\
\hline
\end{tabular}

Table 5: Criteria for positive identification of protein adducts following $\mathrm{HN}-2$ and $\mathrm{HN}-3$ incubation

- Each experiment (triplicate of each HN condition plus a control protein) was performed a total of four times. The final determination for identification of an adduct would require that it be positively identified (as described in the bullet points and table above) in at least three of the four independent experiments. An identified adduct must have been present in each experiment at the same RT $( \pm 0.3 \mathrm{~min})$, else it would not be considered.

The HN-2 and HN-3 adducts identified as described below represent findings that met all of the above-mentioned criteria for positive adduct identification. Figure 25 depicts the data analysis flow utilized for these experiments. The specific parameters in BioConfirm were selected utilizing recommendations from the manufacturer and assistance from the software familiarization guide. 


\section{Open data file}

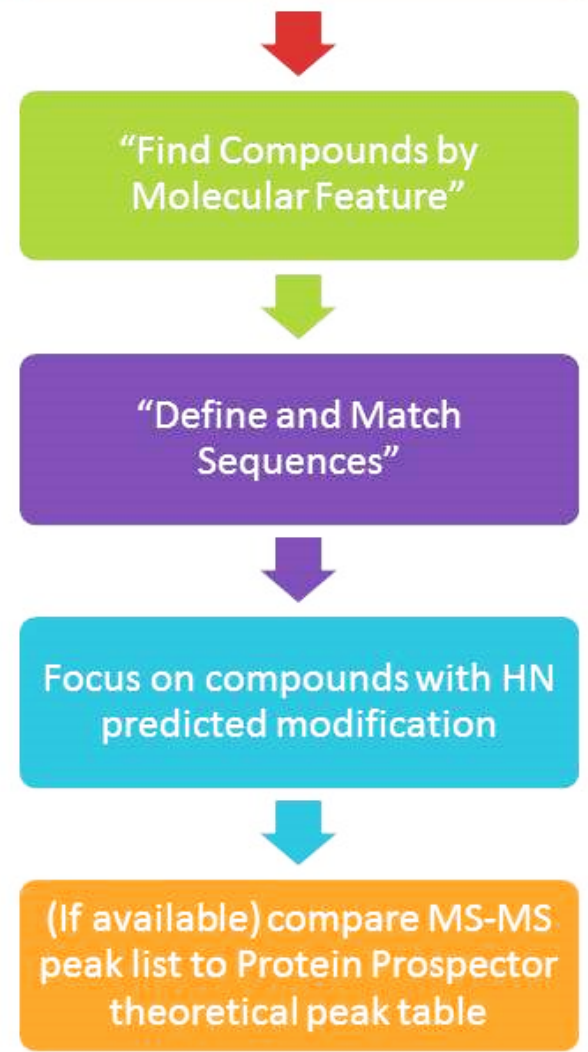

- Parameters as specified in Appendix 5

- Compound list will contain all identified "compounds" based on set criteria

- Specify proteolytic enzyme utilized (trypsin) and maximum number of missed cleavages (2)

- $\quad$ Allowed predicted modifications as specified in Appendix 5

- Of these compounds, determine which have MS-MS data available for analysis

- Obtain peak list for HN-modified compounds with MS-MS data

- Compare obtained peak list values to those obtained through Protein Prospector

- Protein Prospector specifics as specified in Appendix 7

Figure 25: Data processing workflow utilizing BioConfirm and Protein Prospector

\subsubsection{Direct Injection Analysis of Full Proteins}

Initial direct injection experiments were performed in order to determine the minimum excess concentration of $\mathrm{HN}$ required for an observable shift in both $\mathrm{Hb}$ and $\mathrm{HSA}$ deconvoluted masses. Despite observable mass shifts in the direct injection samples, the only method of confirmation was via protein digestion (discussed below). Nevertheless, direct injection analysis provided a rapid screening method to determine exposure-response relationships with the full proteins. Deconvolution parameters are detailed in Appendix 5. 
Direct injection analysis of native $\mathrm{Hb}$ yielded two individual protein peaks - one representing the alpha $(\alpha)$ subunit with a molecular weight of $15,126 \mathrm{Da}$, and the second representing the beta $(\beta)$ subunit with a molecular weight of $15,867 \mathrm{Da}$. Additionally, the MS spectral data included a peak representative of the heme group (molecular weight of $616 \mathrm{Da}$ ). Deconvoluted masses for the native $\alpha$ and $\beta$ subunits of $\mathrm{Hb}$ were consistent over time and accurate when compared to theoretical literature values for subunit mass, ${ }^{135,136}$ demonstrating the usefulness of the analysis method and deconvolution parameters. Based on the direct injection experiments for $\mathrm{Hb}$ incubated with $\mathrm{HN}-2$ and $\mathrm{HN}-3$, a molar excess of 100:1 was deemed necessary for further adduction experiments with $\mathrm{Hb}$.

Direct injection analysis of HSA yielded results that were less consistent, but expected. HSA is a much larger protein $(66,473 \mathrm{Da})$ and as such, produces a more complicated MS spectrum for deconvolution purposes. In addition, due to other potential post-translational modifications and genetic variability, the molecular weight of HSA will vary. Commercial vendors provide an expected range for the deconvoluted mass of HSA that varies up to $200 \mathrm{Da}$ higher than the projected mass based on amino acid composition. This variation was taken into consideration when analyzing HSA, and unmodified HSA did indeed fit within this window each time using the same lot of lyophilized HSA. Based on the direct injection analysis of HSA incubated with $\mathrm{HN}-2$ and $\mathrm{HN}-3$, a molar excess of 50:1 HN was deemed necessary for observable mass shift as a result of adduction for further digestion experiments. 


\subsubsection{HN-2 Adduction to Hemoglobin}

After exposure to $\mathrm{HN}-2$, a total of three novel, specific His adducts were positively identified. Of these three identified His modifications, two were on the $\beta$ chain and the third on the $\alpha$ chain. In addition, adduction to $\mathrm{N}$-terminal Val residues on both the $\alpha$ and $\beta$ chain were confirmed through these studies.

Figure 26 shows a representative MS-MS spectrum for the confirmation of the $\alpha-\mathrm{N}$ terminal adduction on the tryptic fragment VLSPADKTNVK. While the modification of the Nterminus has been previously published, ${ }^{94}$ there has not been MS-MS confirmation of this adduct on the reactive site, nor has there been any specification as to which $\mathrm{N}$-terminus ( $\alpha$ or $\beta$ ) was modified. In the case of $\mathrm{HN}-2$, reproducible modification of both $\mathrm{N}$-termini was observed.

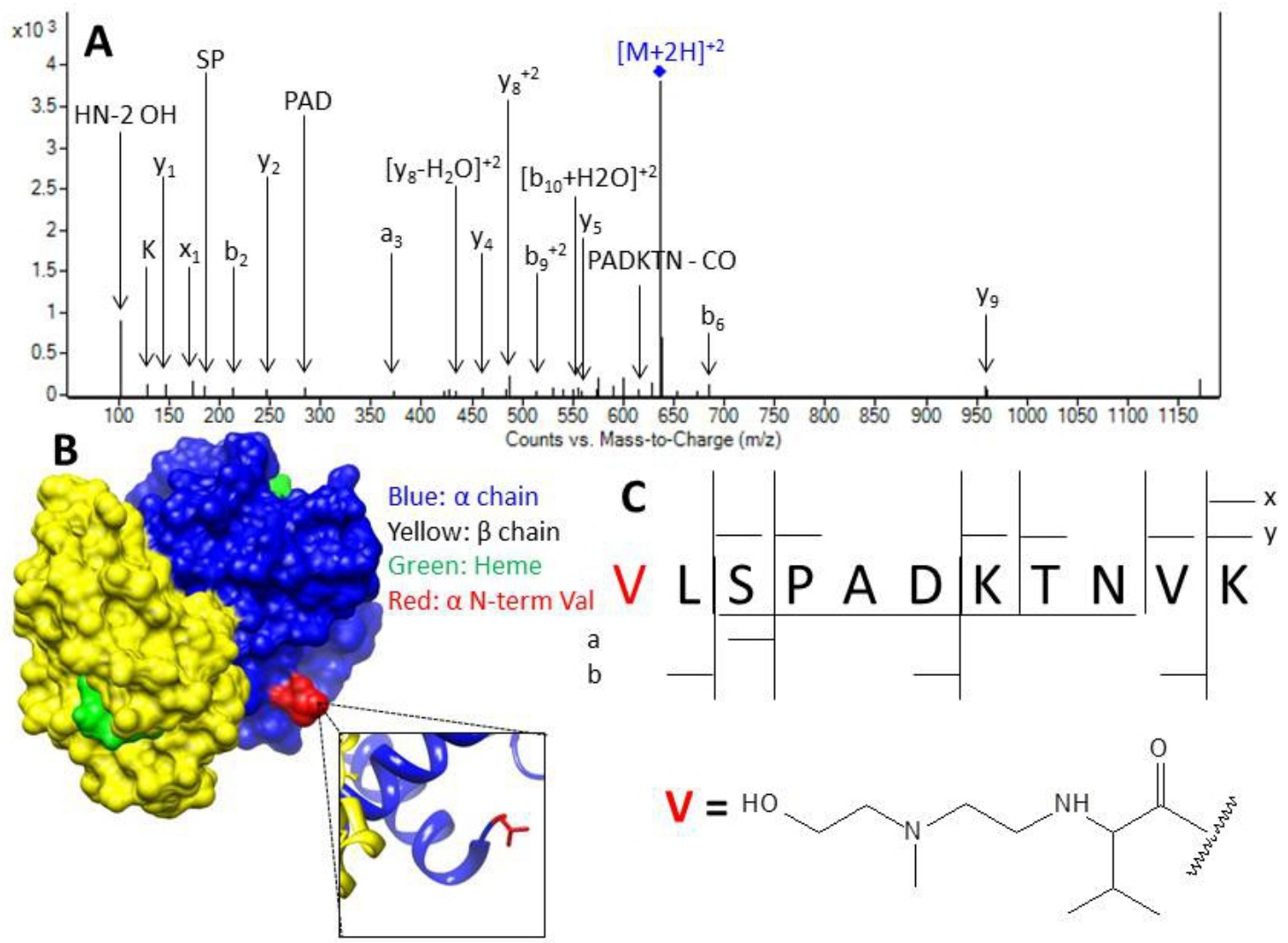

Figure 26: MS-MS identification of $\mathrm{Hb} \alpha \mathrm{N}$-terminal adduction by HN-2. (A) Representative MS-MS spectrum of tryptic peptide. (B) Space-filled model of $\alpha$ (blue) and $\beta$ (yellow) chains of $\mathrm{Hb}$, with adducted residue ( $\alpha$ N-term) highlighted in red. (C) Fragmentation diagram for MS-MS data, with adducted $\mathrm{N}$-terminal structure. 
Figure 27 demonstrates $\mathrm{HN}-2$ adduction to the $\beta$-N-terminus on the tryptic fragment

VHLTPEEK. As previously mentioned, this is the first documented MS-MS confirmation of HN-

2 to this adduction site.

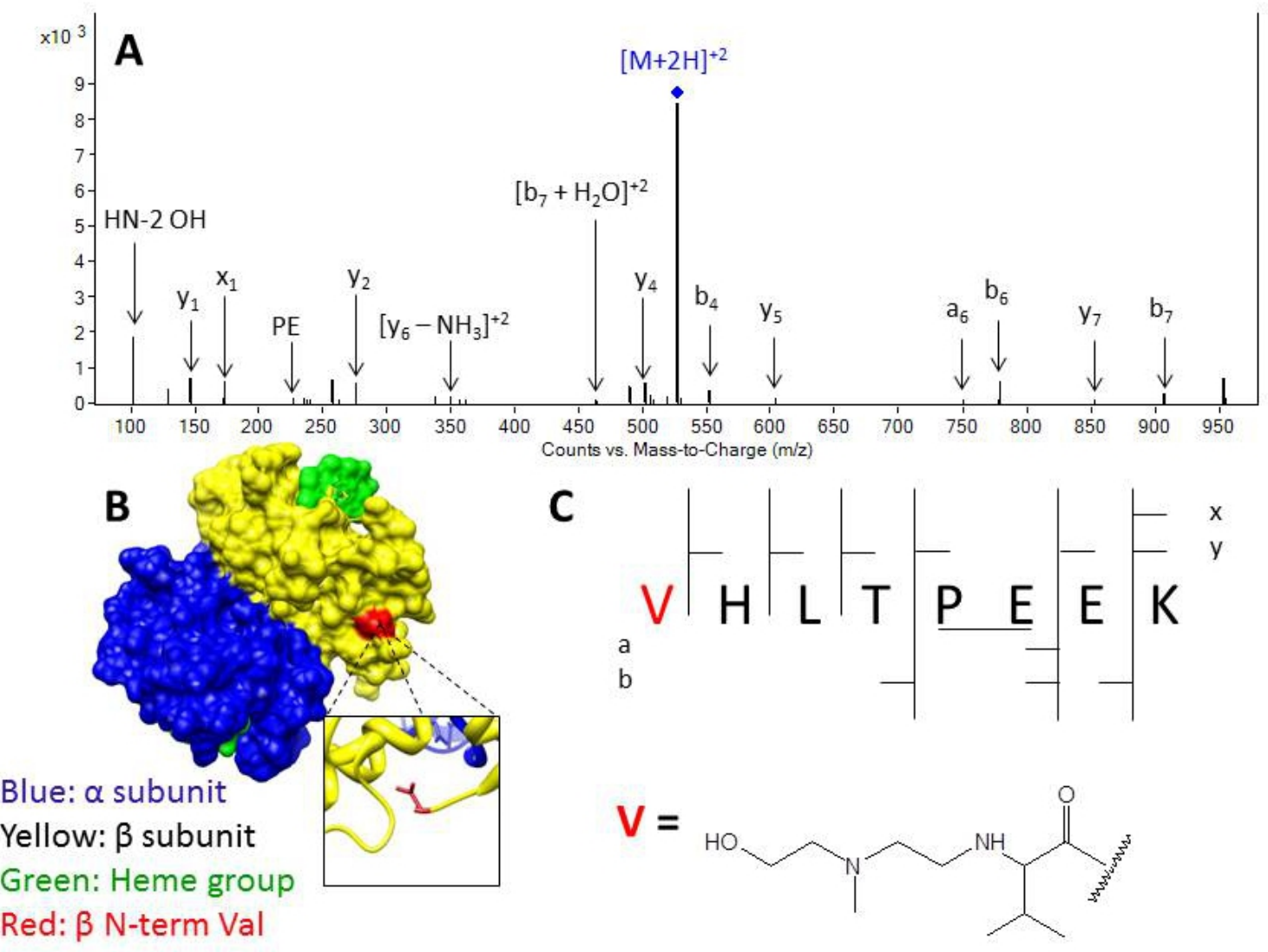

Figure 27: MS-MS identification of Hb $\beta$ N-terminal adduction by HN-2. (A) Representative MS-MS spectrum of tryptic peptide. (B) Space-filled model of $\alpha$ (blue) and $\beta$ (yellow) chains of $\mathrm{Hb}$, with adducted residue ( $\beta$ N-term) highlighted in red. (C) Fragmentation diagram for MS-MS data, with adducted $\mathrm{N}$-terminal structure. 
The three novel protein adducts on $\mathrm{Hb}$ for $\mathrm{HN}-2$ were all His modifications. Figure 28 demonstrates the HN-2 modification of His- $50 \alpha$, the only novel reproducible modification observed for the $\alpha$ subunit via the tryptic fragment TYPFHFDLSHGSAQVK.

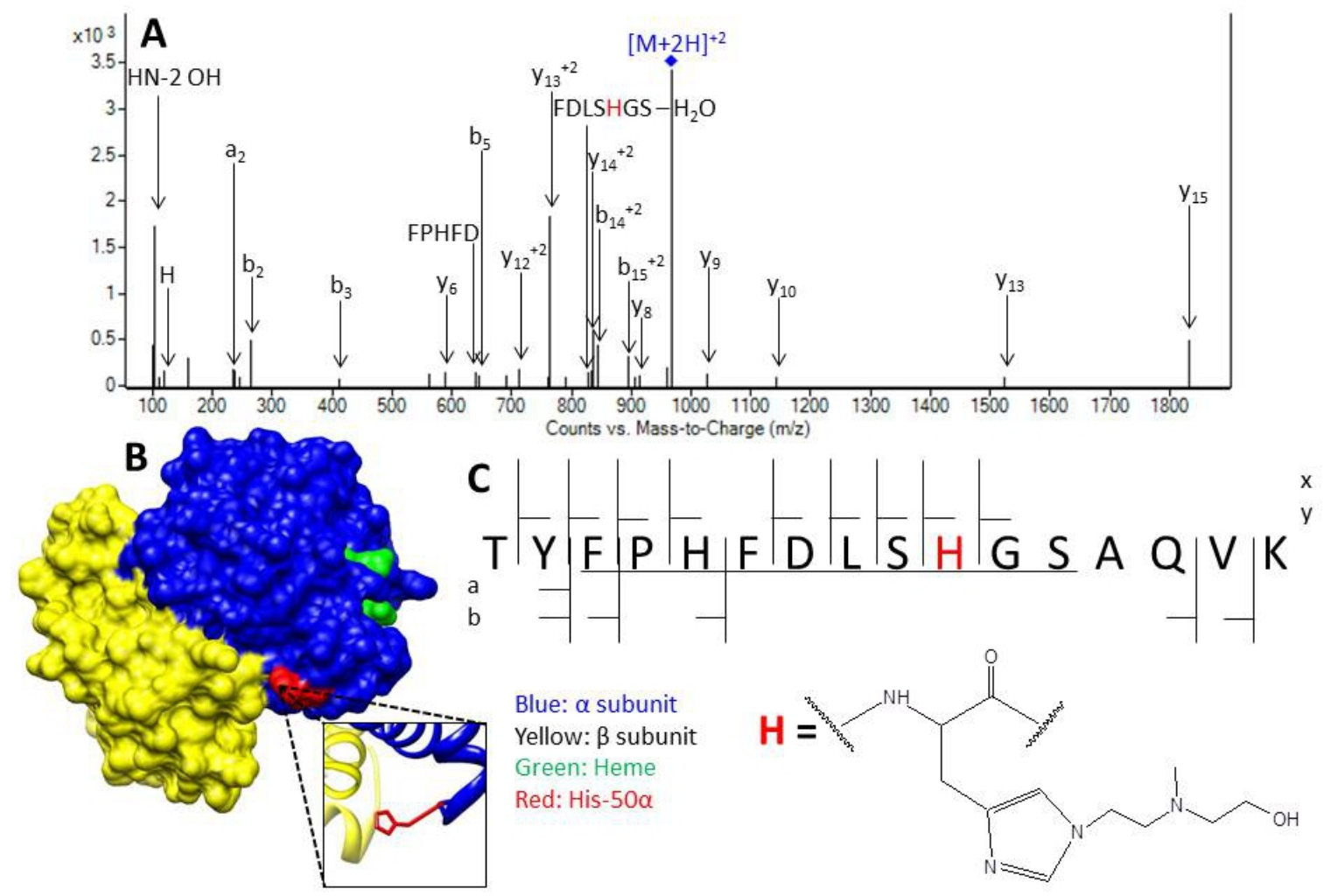

Figure 28: MS-MS identification of Hb His-50 $\alpha$ adduction by HN-2. (A) Representative MS-MS spectrum of tryptic peptide. (B) Space-filled model of $\alpha$ (blue) and $\beta$ (yellow) chains of Hb, with adducted residue (His-50 $\alpha$ ) highlighted in red. (C) Fragmentation diagram for MS-MS data, with adducted His residue structure. 
The other two previously undocumented $\mathrm{HN}-2$ protein His adducts were found on the $\beta$ chain of $\mathrm{Hb}$. The first is His-77 $\beta$, on the tryptic fragment VLGAFSDGLAHLDNLK.

Representative MS-MS fragmentation may be seen in Figure 29.

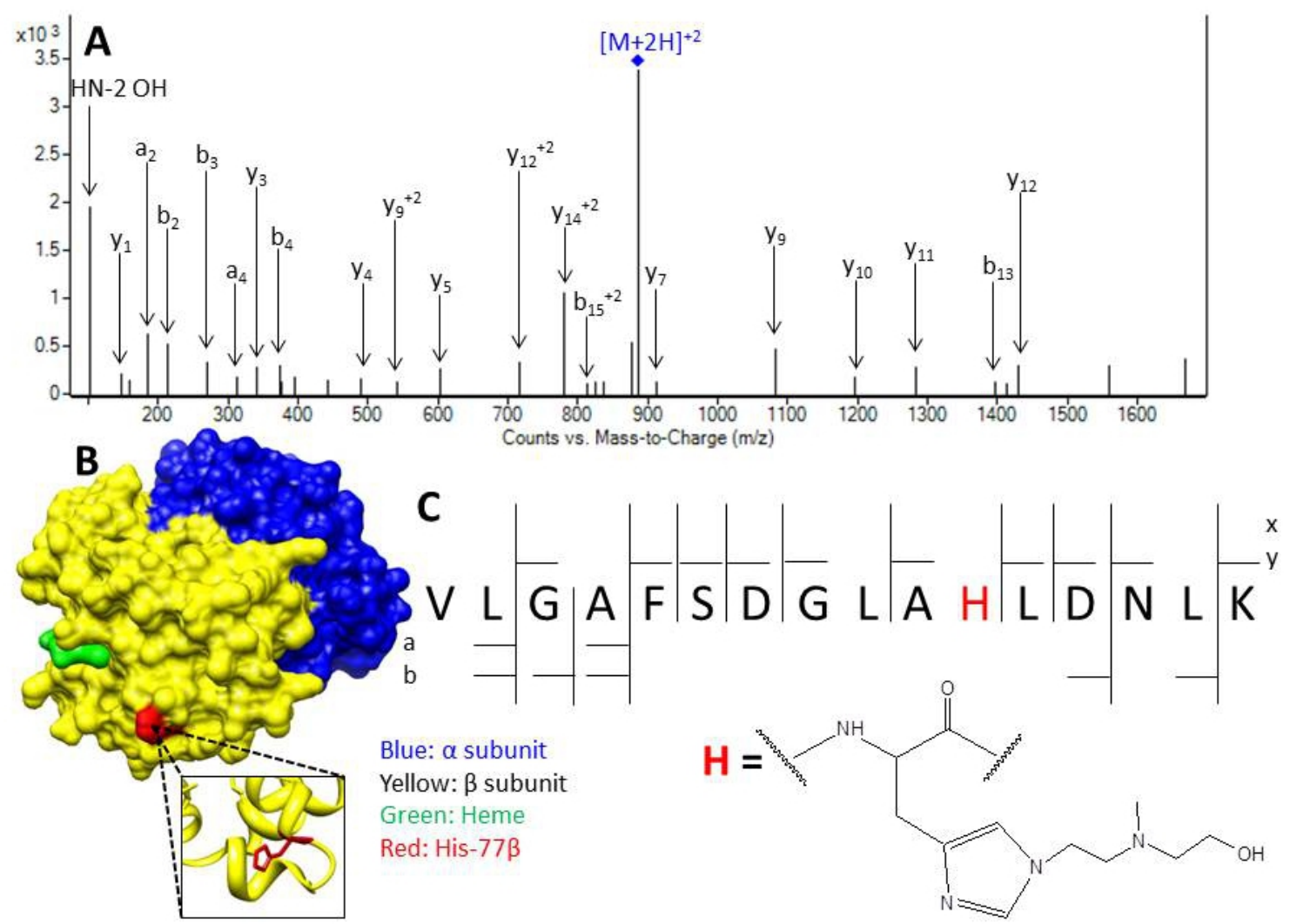

Figure 29: MS-MS identification of Hb His-77 $\beta$ adduction by HN-2. (A) Representative MS-MS spectrum of tryptic peptide. (B) Space-filled model of $\alpha$ (blue) and $\beta$ (yellow) chains of Hb, with adducted residue (His-77 $\beta$ ) highlighted in red. (C) Fragmentation diagram for MS-MS data, with adducted His structure. 
The final HN-2 adduct is present on His-143ß, on the tryptic fragment

VVAGVANALAHK. Representative MS-MS fragmentation may be seen in Figure 30.
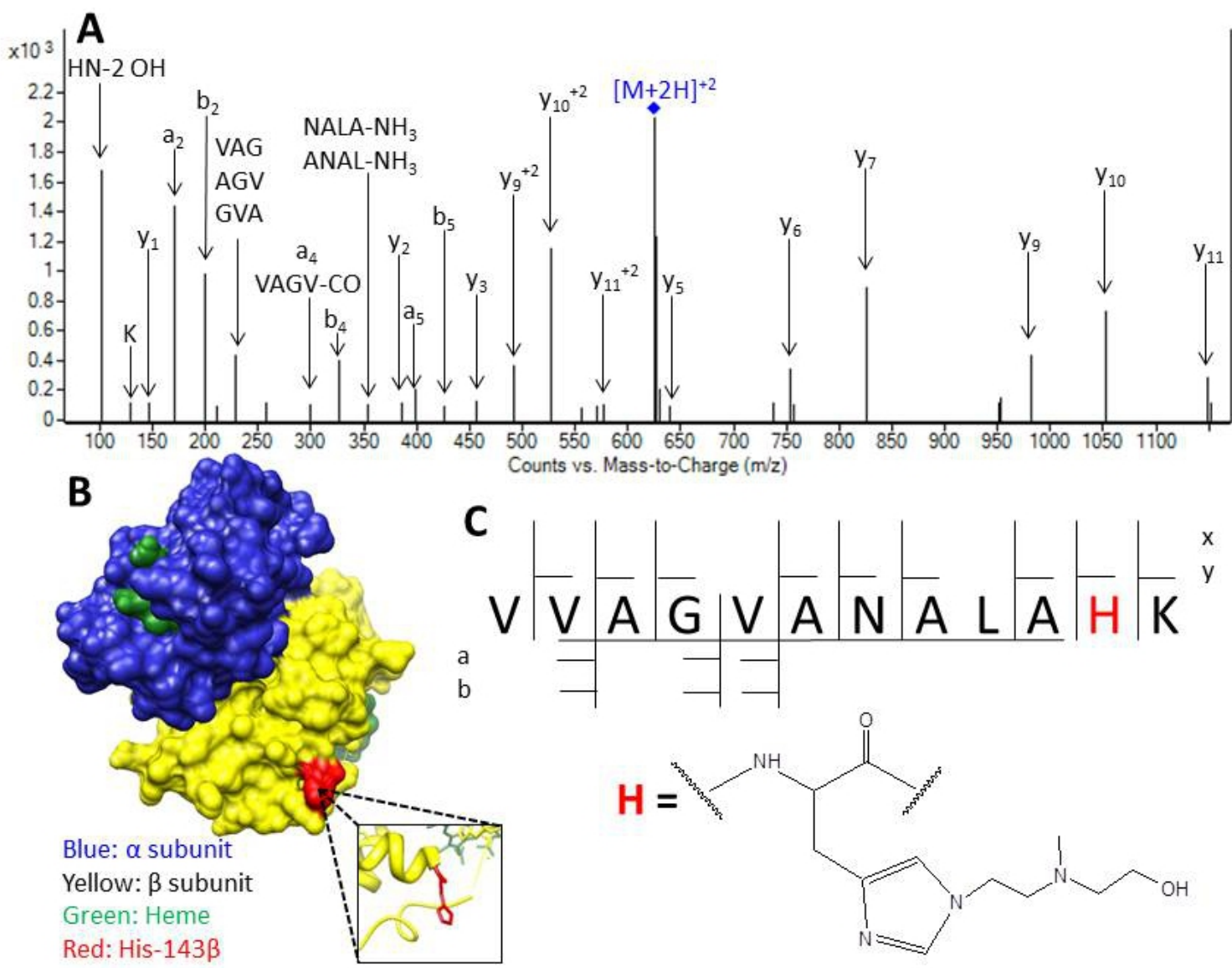

Figure 14: MS-MS identification of Hb His-143 $\beta$ adduction by HN-2. (A) Representative MS-MS data of tryptic peptide. (B) Space-filled model of $\alpha$ (blue) and $\beta$ (yellow) chains of $\mathrm{Hb}$ with adducted residue (His-143ß) highlighted in red. (C) Fragmentation diagram for MS-MS data, with adducted His structure.

\subsubsection{HN-3 Adduction to Hemoglobin}

$\mathrm{HN}-3$ adduction to $\mathrm{Hb}$ produced novel adducts on the identical His residues as observed for $\mathrm{HN}-2$, a result that was expected given previous results obtained from model peptide studies. However, only one N-terminal modification by HN-3 was positively identified via the criteria listed above. Figure 31 shows the MS-MS fragmentation for $\mathrm{HN}-3$ adduction of the $\beta$-N-terminus via the tryptic peptide VHLTPEEK. While adduction to the $\alpha$-N-terminus by HN-3 was observed 
in some samples, it did not meet the strict criteria set above and was therefore not included in the results.
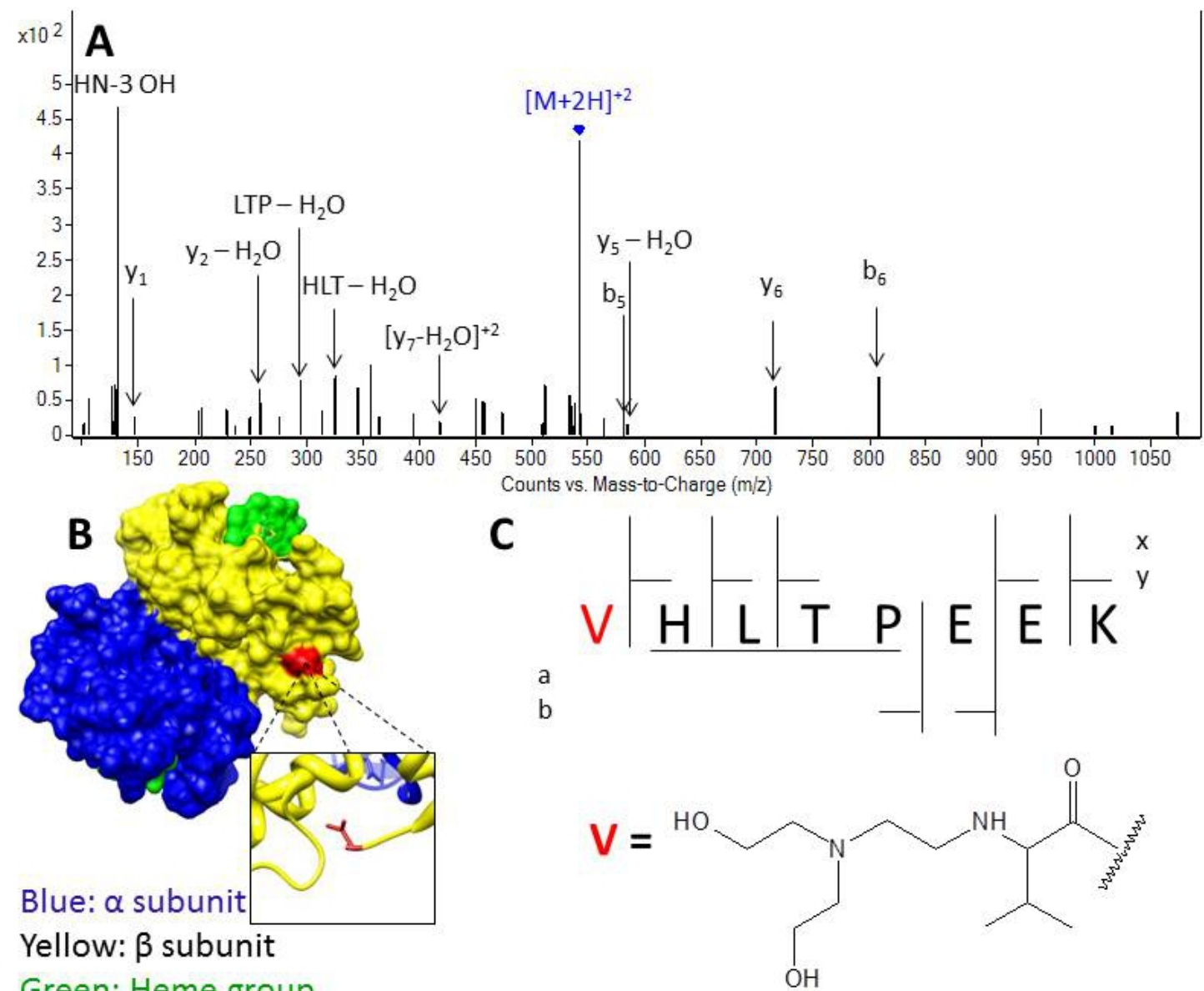

Green: Heme group

Red: $\beta$ N-term Val

Figure 31: MS-MS identification of Hb $\beta$ N-terminal adduction by HN-3. (A) Representative MS-MS spectrum of tryptic peptide. (B) Space-filled model of $\alpha$ (blue) and $\beta$ (yellow) chains of $\mathrm{Hb}$, with adducted residue ( $\beta$ N-term) highlighted in red. (C) Fragmentation diagram for MS-MS data, with adducted $\mathrm{N}$-terminal structure. 
Figure 32 demonstrates the results of $\mathrm{HN}-3$ adduction to His-50 $\alpha$ via the tryptic fragment TYPFHFDLSHGSAQVK.
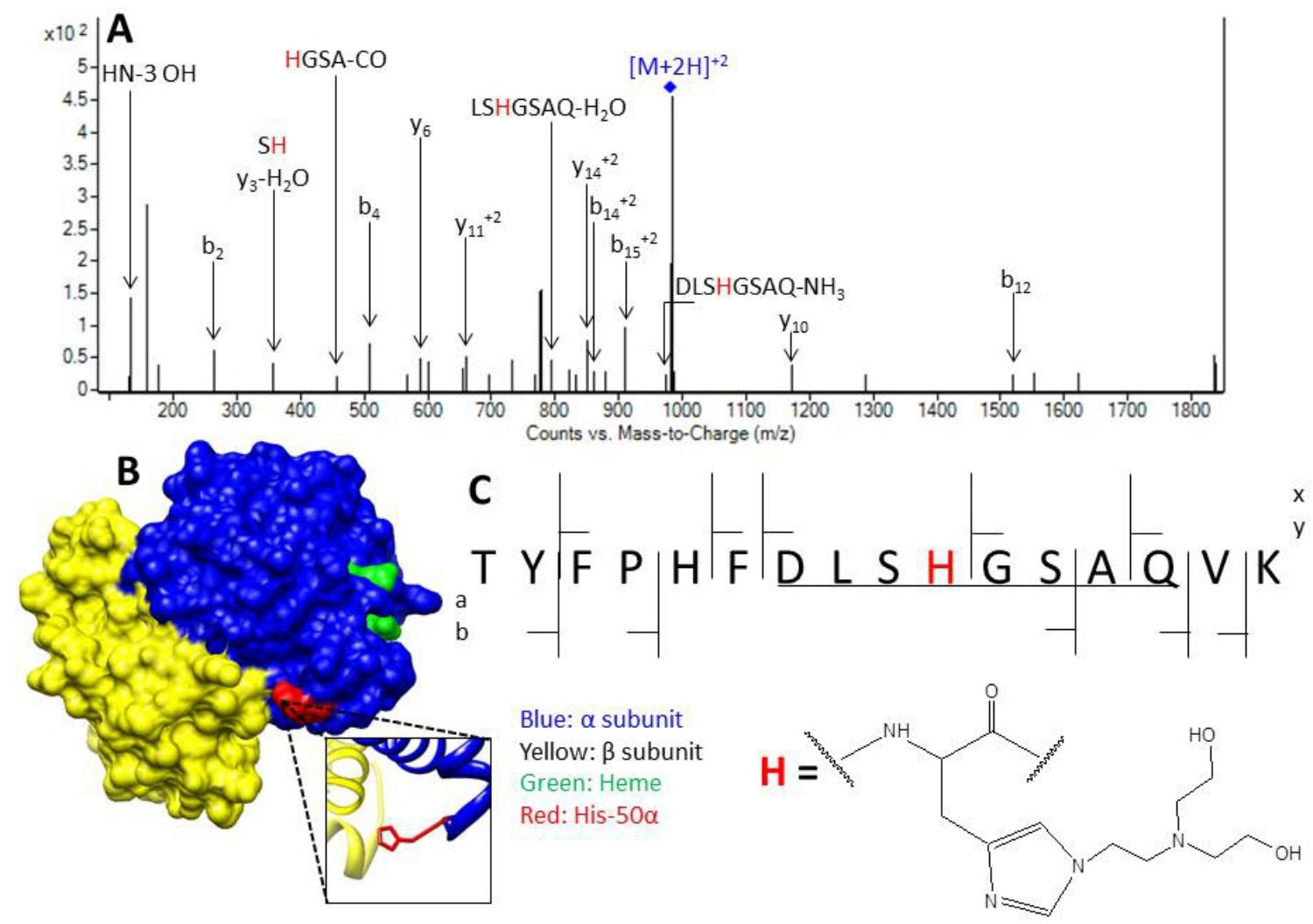

Figure 32: MS-MS identification of Hb His-50 $\alpha$ adduction by HN-3. (A) Representative MS-MS spectrum of tryptic peptide. (B) Space-filled model of $\alpha$ (blue) and $\beta$ (yellow) chains of $\mathrm{Hb}$, with adducted residue (His-50 $\alpha$ ) highlighted in red. (C) Fragmentation diagram of MS-MS data, with adducted His structure. 
Figure 33 demonstrates representative results of HN-3 adduction to His-77 $\beta$ via the tryptic fragment VLGAFSDGLAHLDNLK.
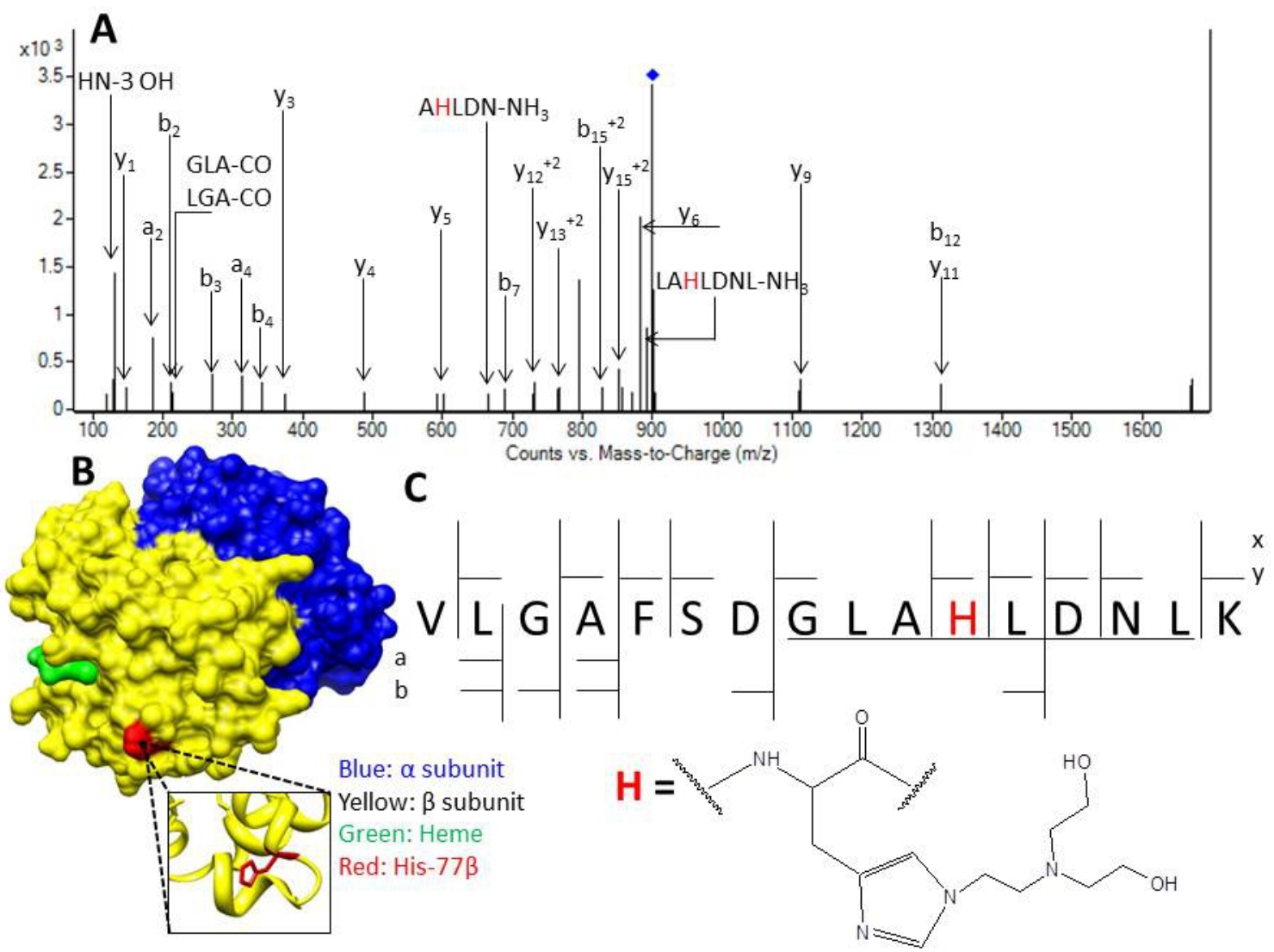

Figure 33: MS-MS identification of Hb His-77 $\beta$ adduction by HN-3. (A) Representative MS-MS spectrum of tryptic peptide. (B) Space-filled model of $\alpha$ (blue) and $\beta$ (yellow) chains of $\mathrm{Hb}$, with adducted residue (His-77 $\beta$ ) highlighted in red. (C) Fragmentation diagram for MS-MS data, with adducted His structure. 
Finally, Figure 34 demonstrates adduction of HN-3 to His-143ß via the tryptic fragment VVAGVANALAHK.

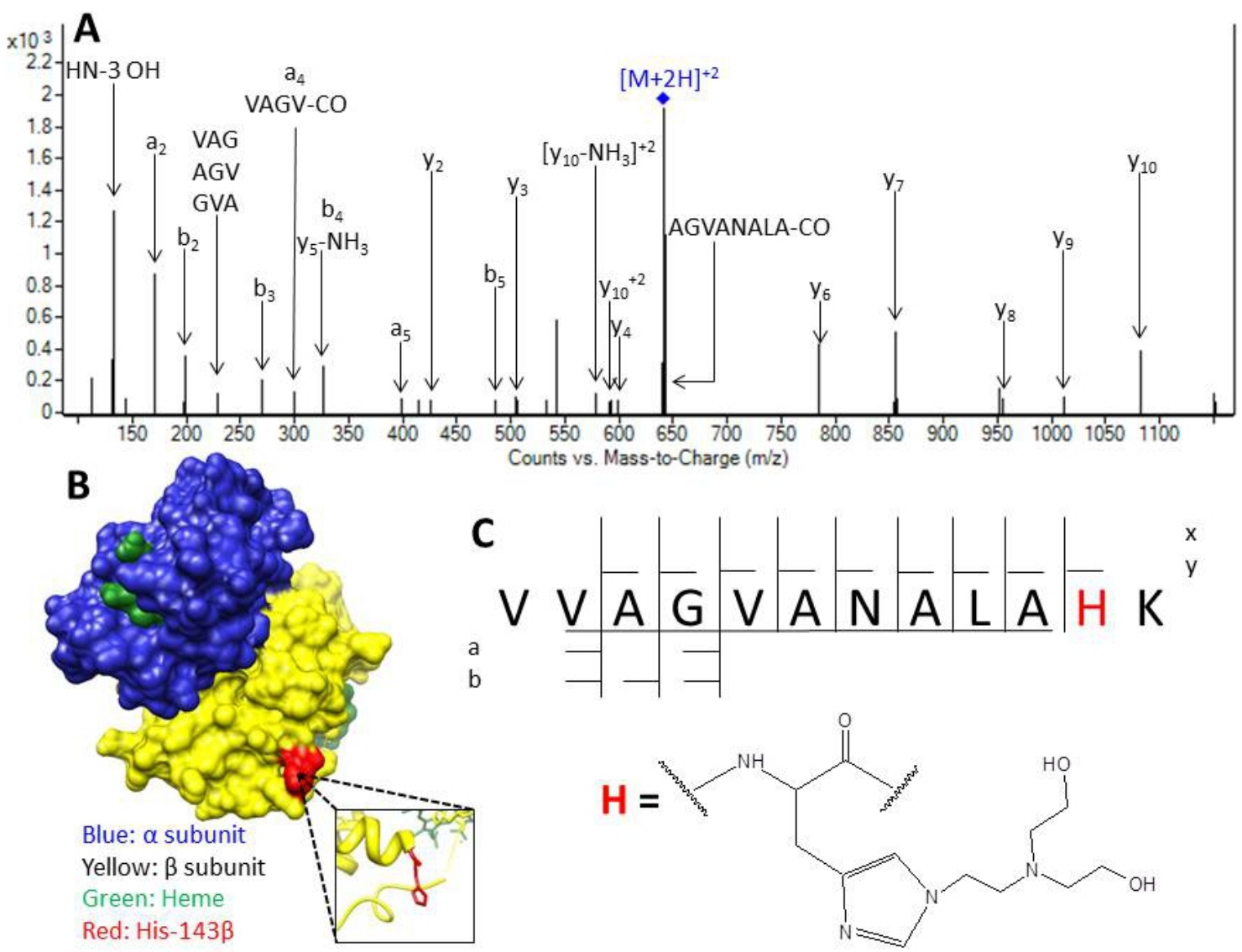

Figure 34: MS-MS identification of Hb His-143ß adduction by HN-3. (A) Representative MS-MS spectrum of tryptic peptide. (B) Space-filled model of $\alpha$ (blue) and $\beta$ (yellow) chains of $\mathrm{Hb}$, with adducted residue (His-143ß) highlighted in red. (C) Fragmentation diagram for MS-MS data, with adducted His structure.

\subsubsection{HN-2 Adduction to Human Serum Albumin}

Adduction assessment of HSA revealed a total of three novel, specific HN-2 adducts, two of which were Lys modifications, and the third a His modification. In addition, since the Nterminus of a protein is generally considered to be a reactive site (so long as it is not blocked), HN-2 was also determined to adduct to the N-terminal Asp residue of HSA. Figure 35 provides MS-MS confirmation of N-terminal Asp adduction of HSA by HN-2. 


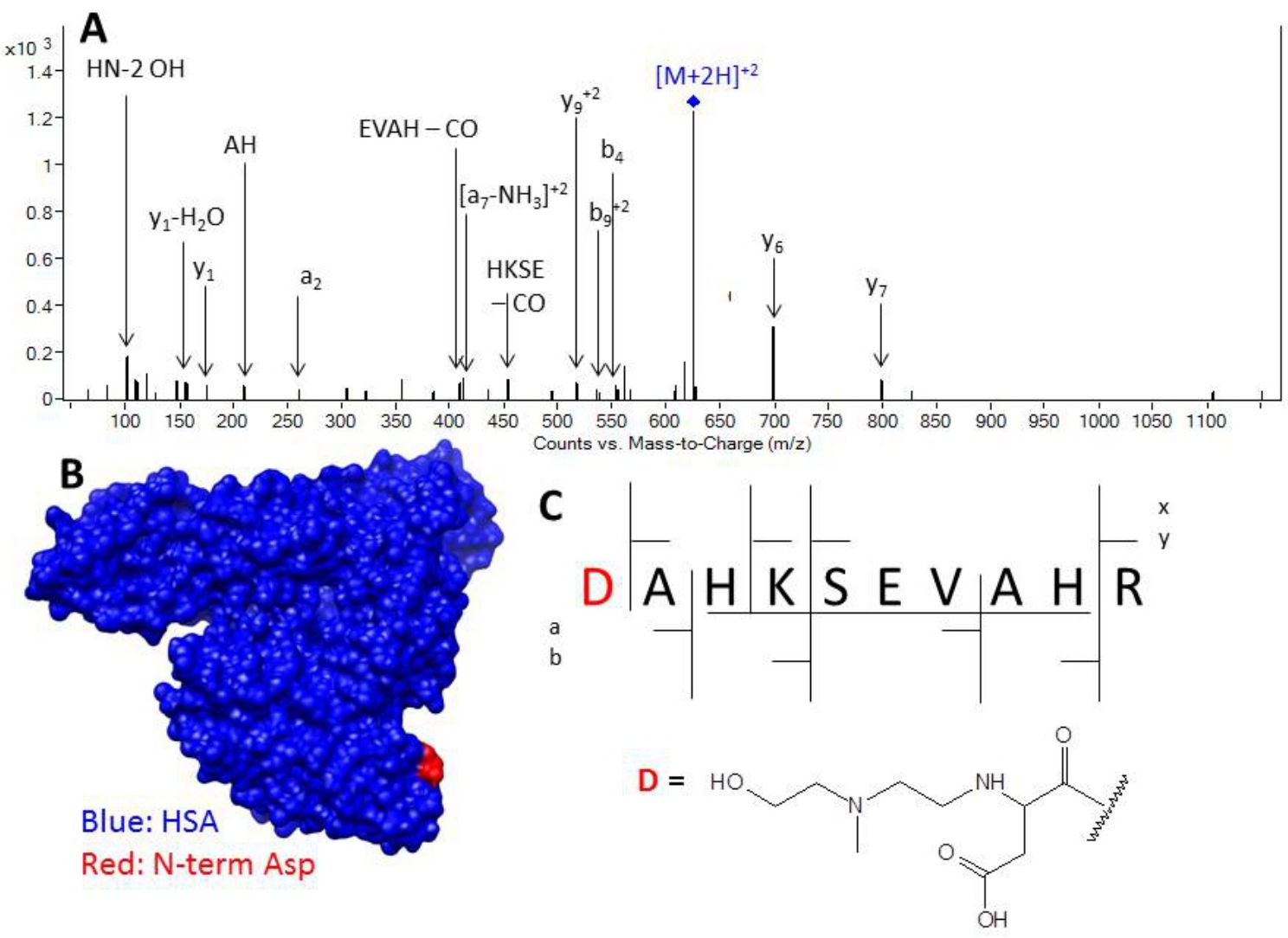

Figure 35: MS-MS identification of HSA N-terminal modification by HN-2. (A) Representative MSMS spectrum of tryptic peptide. (B) Space-filled model of HSA with adducted residue (N-term) highlighted in red. (C) Fragmentation diagram for MS-MS data, with adducted N-terminal structure. 
Two specific HN-2 adducts were identified on HSA on Lys residues. The first may be seen in Figure 36, which demonstrates results obtained from $\mathrm{HN}-2$ adduction to Lys-233 via the tryptic peptide AEFAEVSKLYTDLK.

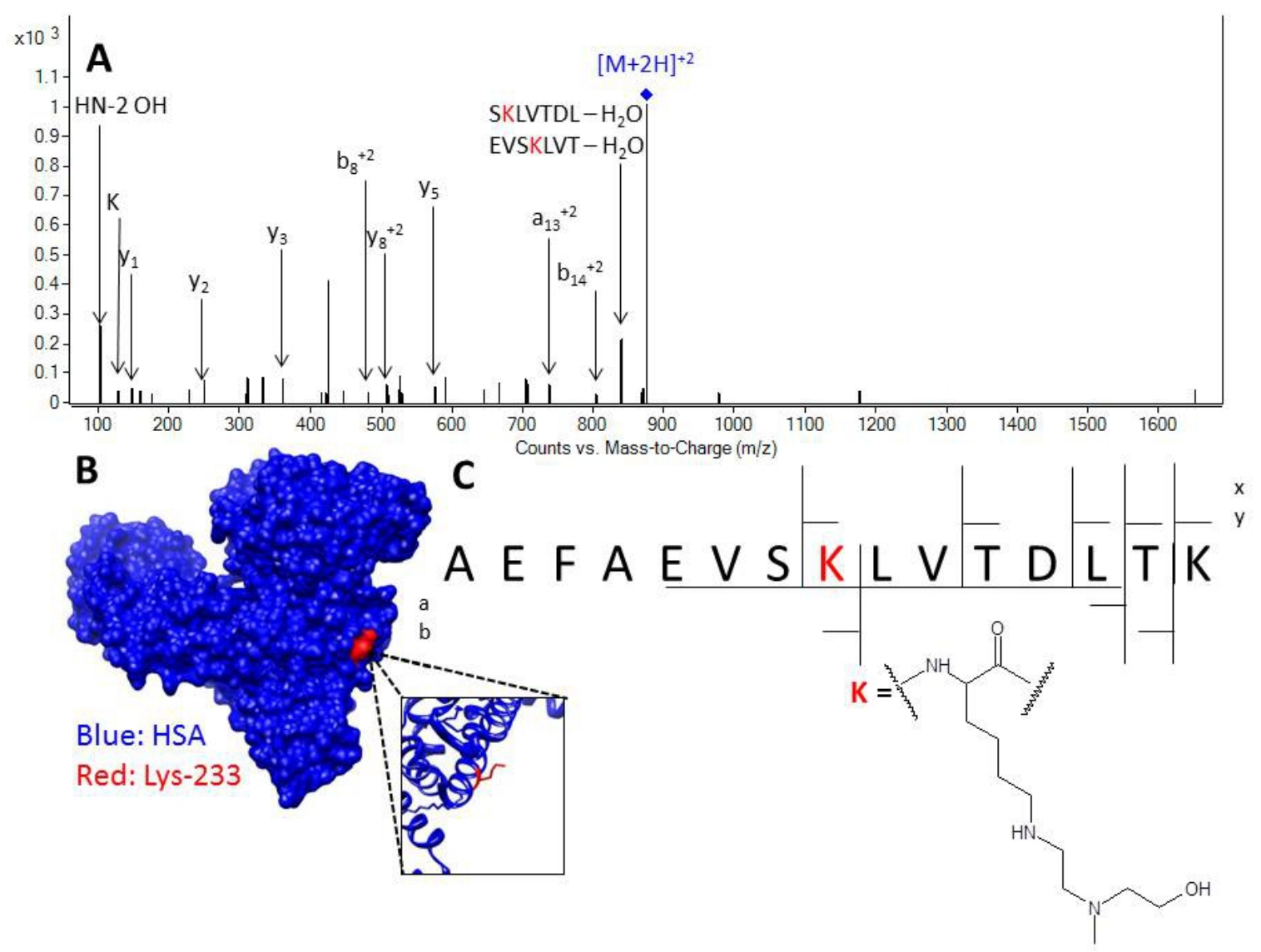

Figure 36: MS-MS identification of HSA Lys-233 adduction by HN-2. (A) Representative MS-MS spectrum of tryptic peptide. (B) Space-filled model of HSA, with adducted residue (Lys-233) highlighted in red. (C) Fragmentation diagram for MS-MS data, with adducted Lys structure. 
The second adducted Lys residue is Lys-317, as seen in Figure 37, via the tryptic fragment DVCKNYAEAK. The data also demonstrates that Cys-316 present in this peptide has been alkylated with iodoacetamide during sample processing. This was expected, as Cys-316 is present in HSA as a disulfide and would thus not be adducted by HN-2 via the protocol utilized in this experiment.

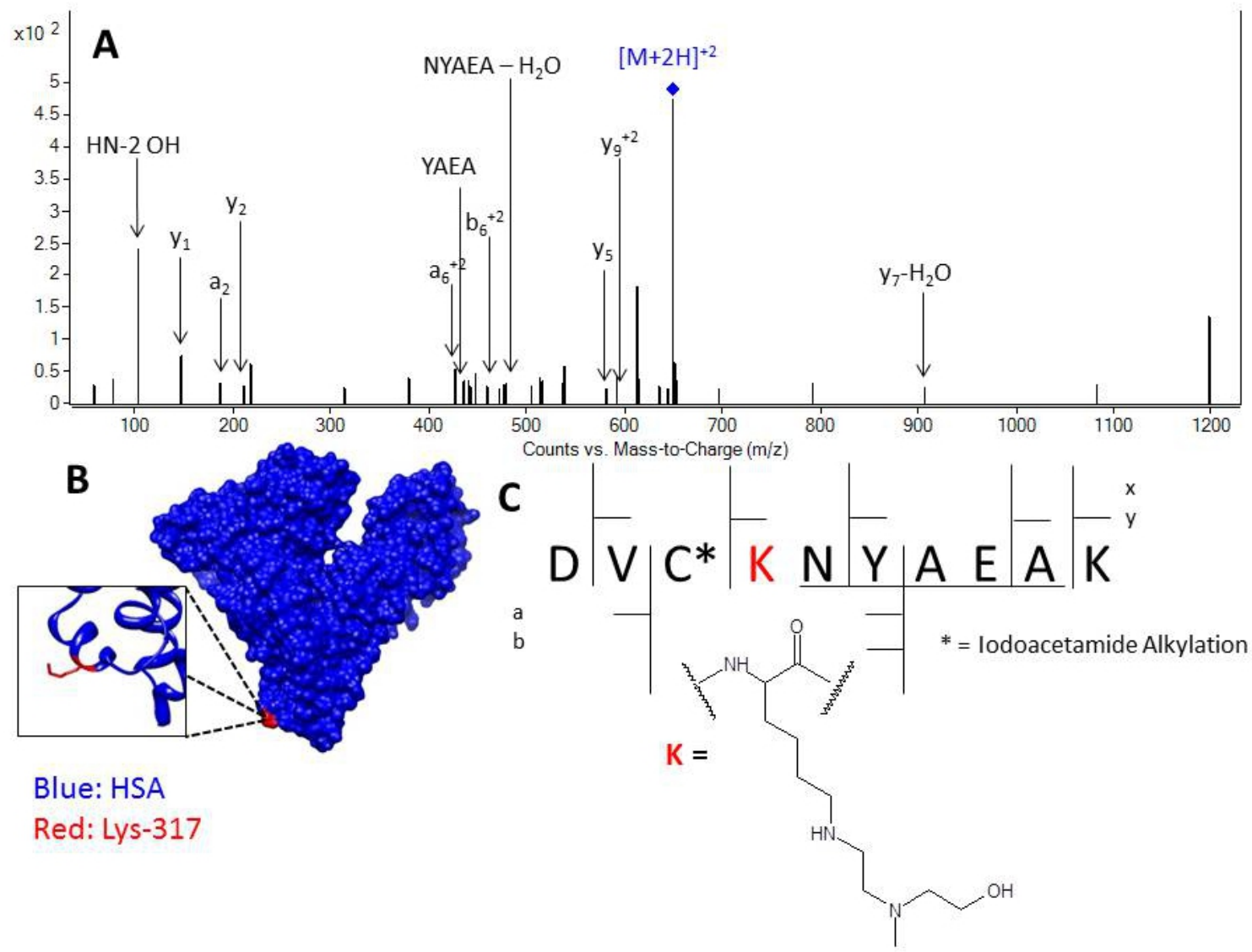

Figure 37: MS-MS identification of HSA Lys-317 adduction by HN-2. (A) Representative MS-MS spectrum of tryptic peptide. (B) Space-filled model of HSA, with adducted residue (Lys-317) highlighted in red. (C) Fragmentation diagram for MS-MS data, with adducted Lys structure. 
Finally, the third novel HN-2 protein adduct identified in this work is His-376, via the tryptic peptide CCAAADPHECYAK. The three Cys residues present in this peptide have all been alkylated by iodoacetamide, which is supported by the MS-MS data seen in Figure 38. As these three Cys are naturally in disulfide bonds in the HSA native structure, they also were not expected to be adduction sites for $\mathrm{HN}-2$.

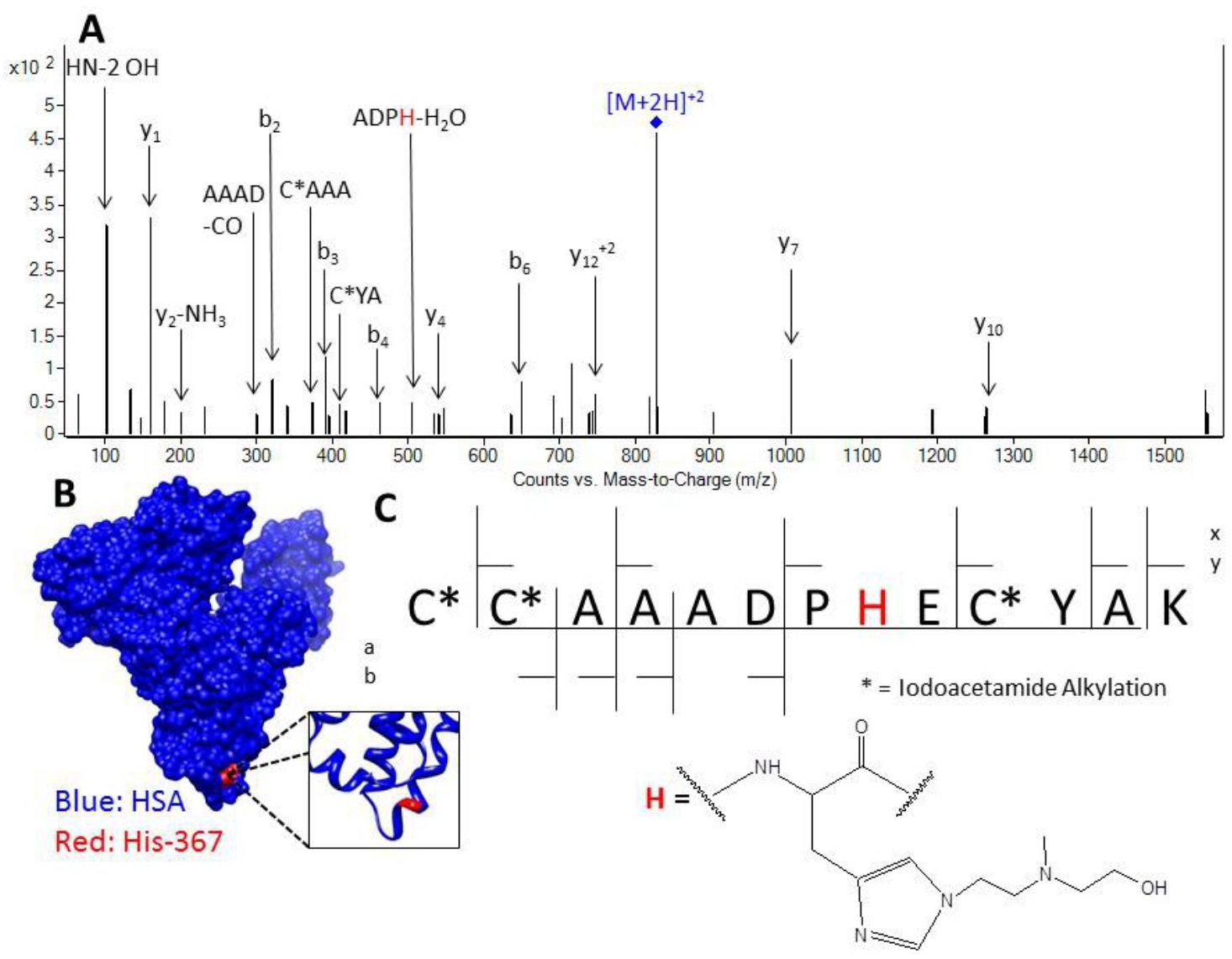

Figure 38: MS-MS identification of HSA His-367 adduction by HN-2. (A) Representative MS-MS spectrum of tryptic peptide. (B) Space-filled model of HSA, with adducted residue (His-367) highlighted in red. (C) Fragmentation diagram for MS-MS data, with adducted His structure. 


\subsubsection{HN-3 Adduction to Human Serum Albumin}

As with $\mathrm{HN}-2, \mathrm{HN}-3$ adduction to HSA yielded two novel, specific protein adducts on Lys residues. In addition, adduction to the $\mathrm{N}$-terminus was also positively identified. Figure 39 demonstrates $\mathrm{HN}-3$ adduction to the N-terminal Asp.

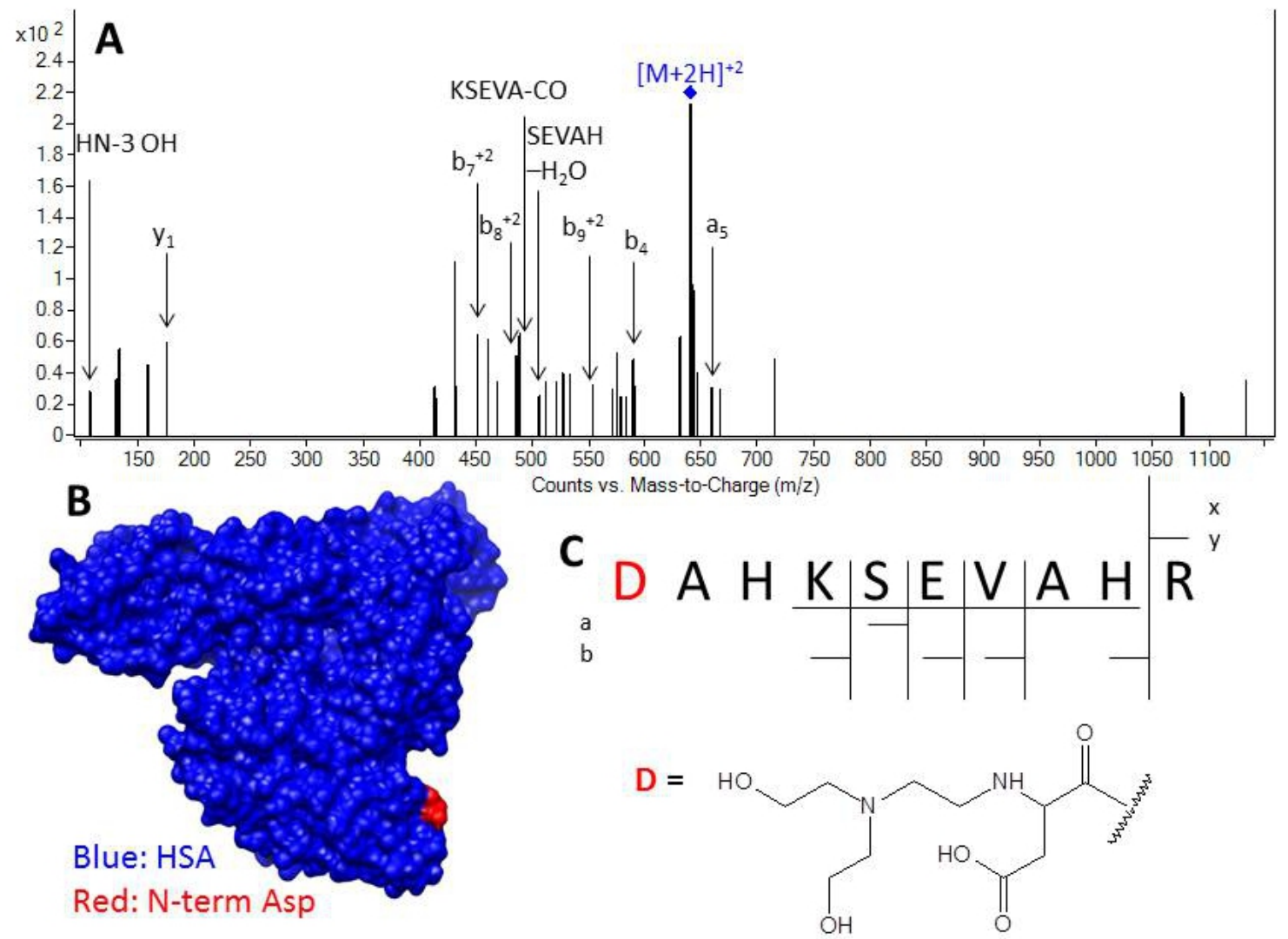

Figure 39: MS-MS identification of HSA N-terminus adduction by HN-3. (A) Representative MS-MS data of tryptic peptide. (B) Space-filled model of HSA, with adducted residue (N-term) highlighted in red. (C) Fragmentation diagram for MS-MS data, with adducted N-terminus structure. 
Figure 40 demonstrates HN-3 adduction to Lys-233 via the tryptic peptide

\section{AEFAEVSKLVTDLK.}

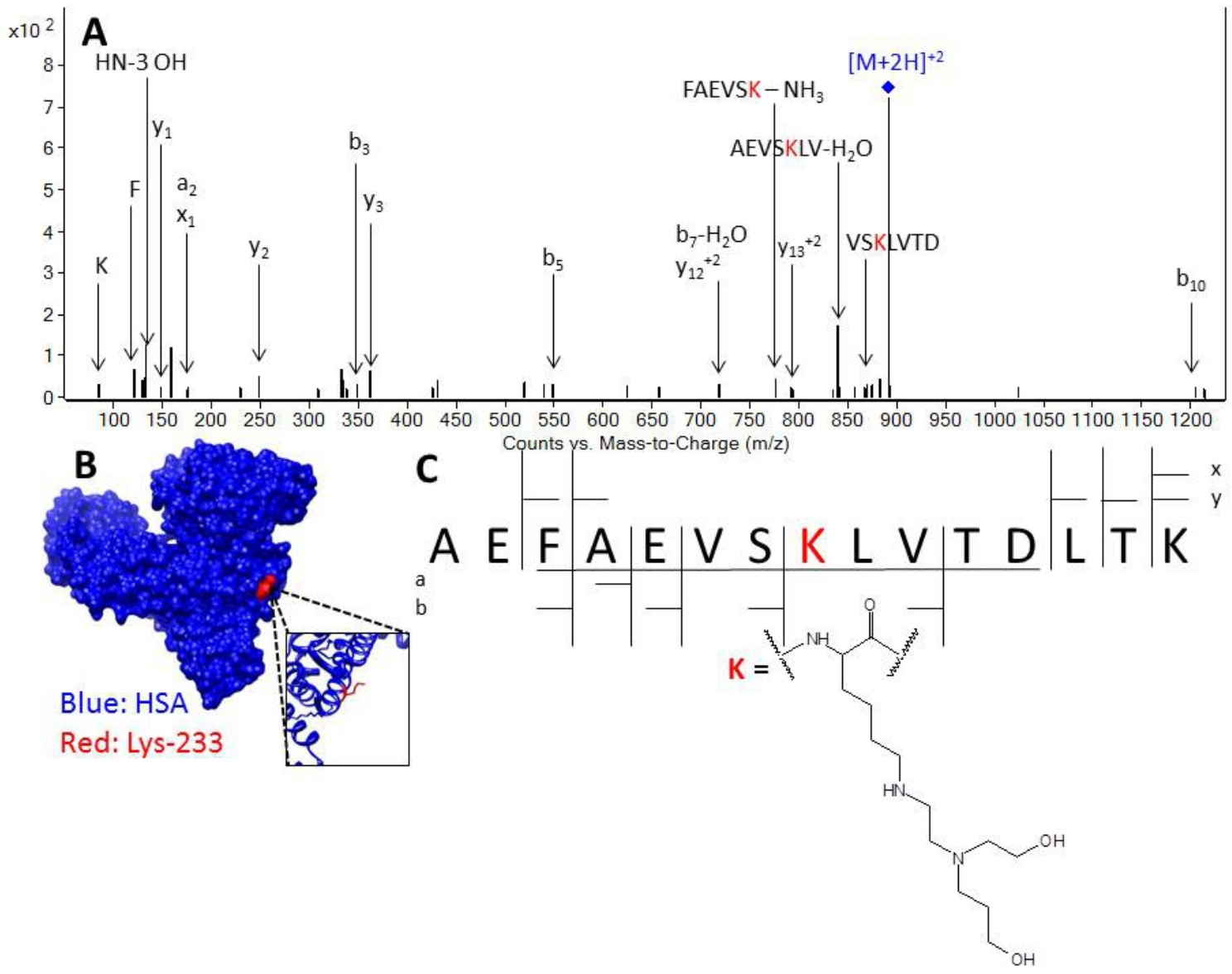

Figure 40: MS-MS identification of HSA Lys-233 adduction by HN-3. (A) Representative MS-MS spectrum of tryptic peptide. (B) Space-filled model of HSA, with adducted residue (Lys-233) highlighted in red. (C) Fragmentation diagram for MS-MS data, with adducted Lys structure. 
Figure 41 demonstrates HN-3 adduction to Lys-317 via the tryptic peptide

DVCKNYAEAK. Cys-116 is shown via MS-MS data to be alkylated by iodoacetamide, as was seen with $\mathrm{HN}-2$.
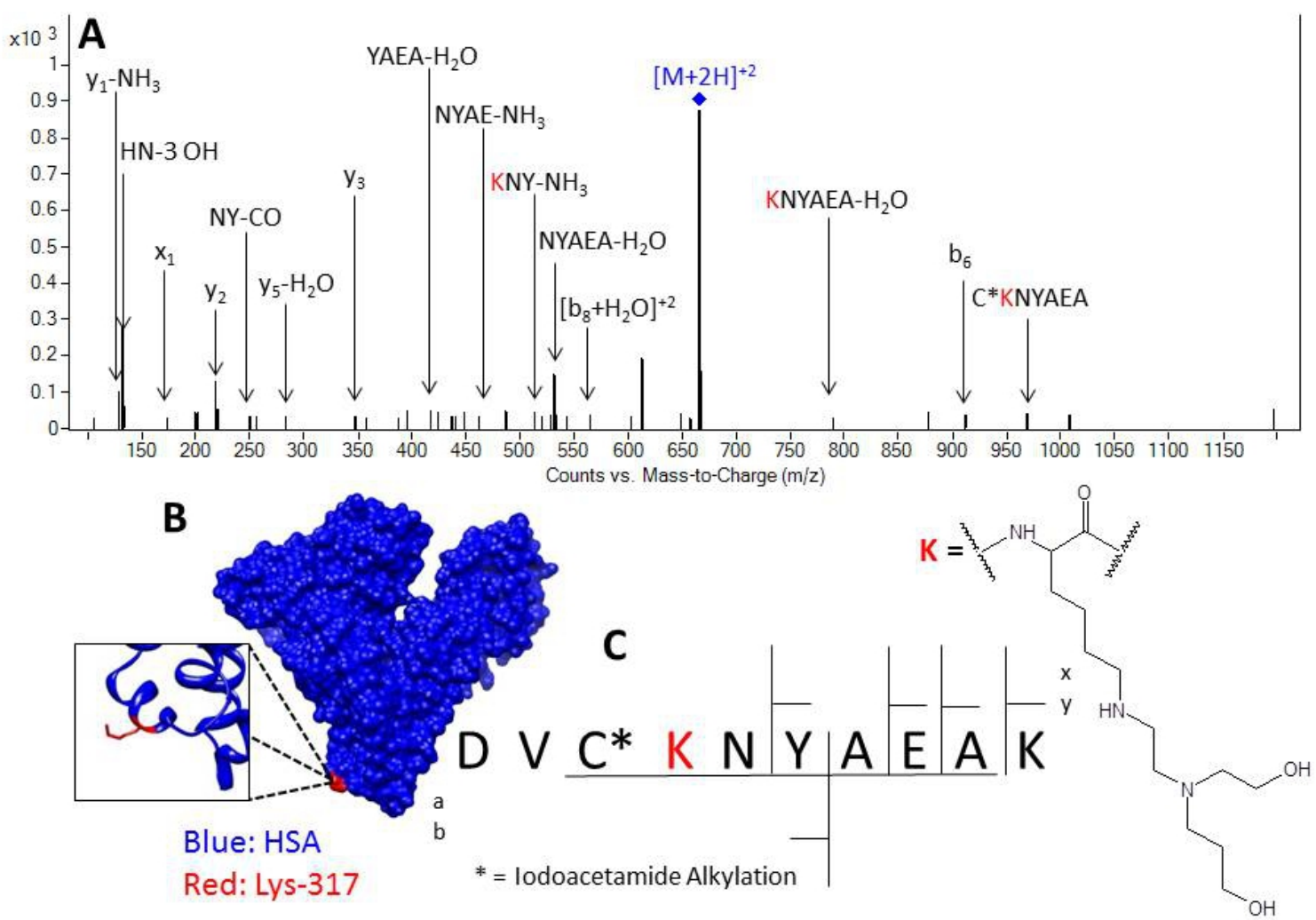

Figure 41: MS-MS identification of HSA Lys-317 adduction by HN-3. (A) Representative MS-MS spectrum of tryptic peptide. (B) Space-filled model of HSA, with adducted residue (Lys-317) highlighted in red. (C) Fragmentation diagram for MS-MS data, with adducted Lys structure.

Adduction to His-367 by HN-3 was observed in several different samples; however, due to the strict criteria set for identification, was not determined to be reproducible for this work. 


\subsubsection{Stability of Full Protein Adducts}

The stability of the identified adducts was determined over a three-week period. As a strict set of criteria had already been employed for initial adduct identification, the requirements for MS-MS data in selecting true positives were not as stringent in these experiments. For the positive identification of adducts during the stability study, it was only deemed necessary for identified adducts to be present at the same RT as previously identified. If MS-MS data were available for identified adducts, it was analyzed by comparison to a theoretical peak list generated by Protein Prospector.

As with previous experiments, triplicate samples were run for each condition. In the tables below, a check mark $(\checkmark)$ corresponds to positive identification of the adduct in at least two of the three samples. An $x(\mathbf{x})$ in the table corresponds to the adduct missing in the peak list in two or more of the triplicate samples. It is important to note that, for each time point, aliquots were removed from the same sample. Therefore, a non-identification at one time point should not necessarily be grounds for characterizing an adduct as unstable. These non-identifications may be a result of variations with the digestion or the Auto MS-MS analysis. An adduct was therefore considered stable if it was present at the three-week time point and was consistently documented during the analysis period.

Table 6 demonstrates the stability of identified HN-2 adducts over a three-week analysis period. All adducts identified were present at the $3 \mathrm{~h}$ and 3 week time points, and therefore were considered stable during the analysis period. 


\begin{tabular}{|c|c|c|c|c|c|}
\hline \multicolumn{7}{|c|}{ Temoglobin } \\
\hline \multirow{2}{*}{ Adduct Site } & \multicolumn{6}{|c|}{ Time Point } \\
\cline { 2 - 6 } & 3hr & 24hr & 1wk & 2wk & 3wk \\
\hline$\alpha$ N-term & $\checkmark$ & $\checkmark$ & $\checkmark$ & $\checkmark$ & $\checkmark$ \\
\hline His-50 $\alpha$ & $\checkmark$ & $\checkmark$ & $\checkmark$ & $\checkmark$ & $\checkmark$ \\
\hline$\beta$ N-term & $\checkmark$ & $\checkmark$ & $\checkmark$ & $\checkmark$ & $\checkmark$ \\
\hline His-77 $\beta$ & $\checkmark$ & $\checkmark$ & $\checkmark$ & $\checkmark$ & $\checkmark$ \\
\hline His-143 $\beta$ & $\checkmark$ & $\checkmark$ & $\checkmark$ & $\checkmark$ & $\checkmark$ \\
\hline
\end{tabular}

\section{Human Serum Albumin}

\begin{tabular}{|c|c|c|c|c|c|}
\hline \multirow{2}{*}{ Adduct Site } & \multicolumn{5}{|c|}{ Time Point } \\
\cline { 2 - 6 } & 3hr & 24hr & 1wk & 2wk & 3wk \\
\hline N-term & $\checkmark$ & $\checkmark$ & x & $\checkmark$ & $\checkmark$ \\
\hline Lys-233 & $\checkmark$ & $\checkmark$ & x & $\checkmark$ & $\checkmark$ \\
\hline Lys-317 & $\checkmark$ & $\checkmark$ & $\checkmark$ & $\checkmark$ & $\checkmark$ \\
\hline His-367 & $\checkmark$ & $\checkmark$ & $\checkmark$ & x & $\checkmark$ \\
\hline
\end{tabular}

Table 6: Stability of identified HN-2 adducts to Hb and HSA over three weeks following adduct formation.

Results for the stability studies for HSA adducts were more variable than with $\mathrm{Hb}$. However, as missing adducts were only noted at a single time point for each adduct, this observation is most likely due to variation with the digestion or the Auto MS-MS trigger, rather than instability of the adduct.

Table 7 demonstrates the stability of identified HN-3 adducts over the three-week analysis period. All adducts identified were present at the $3 \mathrm{~h}$ and 3 week time points, with consistent identification during the analysis period; therefore, these adducts were considered stable during the entire analysis period. 


\begin{tabular}{|c|c|c|c|c|c|}
\hline \multicolumn{6}{|c|}{ Hemoglobin } \\
\hline \multirow{2}{*}{ Adduct Site } & \multicolumn{5}{|c|}{ Time Point } \\
\hline & $3 \mathrm{hr}$ & $24 \mathrm{hr}$ & $1 \mathrm{wk}$ & $2 w k$ & $3 \mathrm{wk}$ \\
\hline His- $50 \alpha$ & $\checkmark$ & $x$ & $\checkmark$ & $\checkmark$ & $\checkmark$ \\
\hline$\beta \mathrm{N}$-term & $\checkmark$ & $\checkmark$ & $\checkmark$ & $\checkmark$ & $\checkmark$ \\
\hline His- $77 \beta$ & $\sqrt{ }$ & $\checkmark$ & $x$ & $\checkmark$ & $\sqrt{ }$ \\
\hline His- $143 \beta$ & $\checkmark$ & $\checkmark$ & $\checkmark$ & $\checkmark$ & $\checkmark$ \\
\hline
\end{tabular}

\section{Human Serum Albumin}

\begin{tabular}{|c|c|c|c|c|c|}
\hline \multirow{2}{*}{ Adduct Site } & \multicolumn{6}{|c|}{ Time Point } \\
\cline { 2 - 6 } & 3hr & 24hr & 1wk & 2wk & 3wk \\
\hline N-term & $\checkmark$ & $\checkmark$ & x & $\checkmark$ & $\checkmark$ \\
\hline Lys-233 & $\checkmark$ & $\checkmark$ & x & $\checkmark$ & $\checkmark$ \\
\hline Lys-317 & $\checkmark$ & $\checkmark$ & $\mathbf{x}$ & $\checkmark$ & $\checkmark$ \\
\hline
\end{tabular}

Table 7: Stability of identified HN-3 adducts to $\mathrm{Hb}$ and HSA over three weeks following adduct formation.

As with Table 6 , cells containing an $\mathbf{x}$ indicate that the adduct could not be identified in at least two of the three samples tested. This phenomenon occurred at a maximum of a single time point per adduction site; therefore, the adducts were in all cases considered stable.

\subsubsection{Discussion}

The experiments presented above have identified several novel and specific protein adducts for $\mathrm{HN}-2$ and $\mathrm{HN}-3$ on $\mathrm{Hb}$ and $\mathrm{HSA}$ that are stable over at least a three-week analysis period. These modifications therefore have potential as specific biomarkers of exposure for these two CWA. All adducts identified in this task were hydroxylated and no longer contained the chlorine atom. The fully hydroxylated form of the adduct should therefore be considered the more relevant structure for biological proteins and for the metabolic assays.

The identified adduction sites for HN-2 show a good overlap with those of HN-3, although fewer adducts were confirmed for HN-3. This is not surprising for two reasons. First, $\mathrm{HN}-2$ has been documented to be more reactive than its HN-3 counterpart. ${ }^{37}$ As such, more adduction would be expected for HN-2. Second, HN-3 has more potential for steric hindrance in 
its reactivity than $\mathrm{HN}-2$. It is possible that the larger $\mathrm{HN}-3$ molecule cannot reproducibly adduct sites that are less accessible, and therefore appears to be less reactive than $\mathrm{HN}-2$.

An additional observation regarding the $\mathrm{HN}-2$ and $\mathrm{HN}-3$ adducts is the location of all the adduction sites. All susceptible residues are located on the surface of these proteins, where they are largely accessible to electrophilic species. Due to this observation, it was of interest to further analyze the surface content of Lys and His residues on the two proteins studied. Figure 42 below demonstrates a space-filled model of $\mathrm{Hb}$, with the surface Lys and His residues highlighted in red and yellow, respectively.
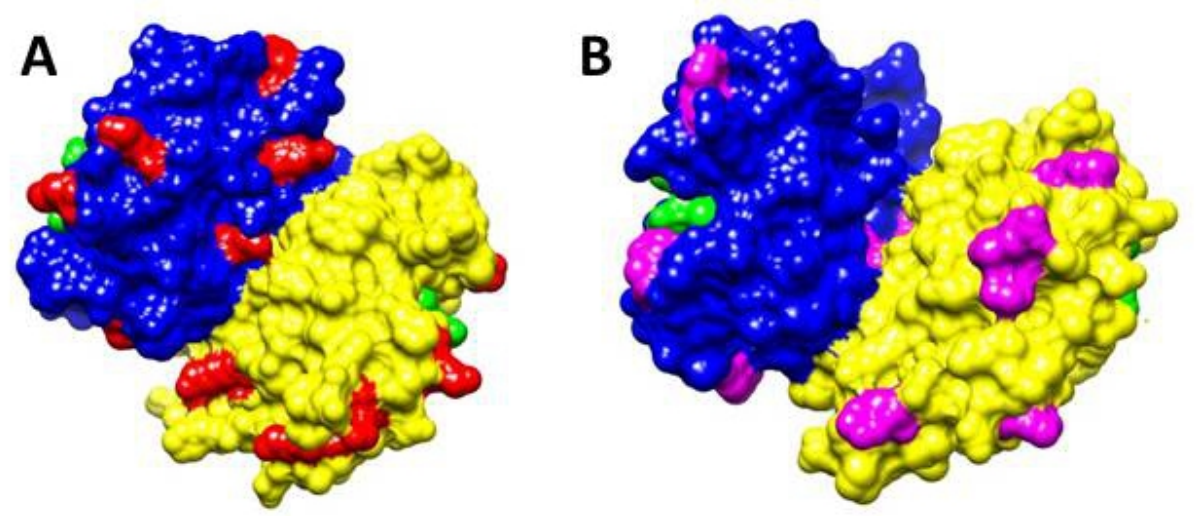

Figure 42: Space-filled model of Hb, with highlighted Lys (A) and His (B) residues. The alpha chain is colored blue, the beta chain is colored yellow, the red resides represent the outer Lys residues, and the pink residues represent the outer His residues.

The figure above suggests that there are several outer Lys residues (red); however, only His modifications were observed as reproducibly adducted by HN-2 and HN-3. The most likely explanation is the lower $\mathrm{pK}_{\mathrm{a}}$ of His residues at $\mathrm{pH} 7.4$ as compared to Lys residues - despite the larger number of surface Lys residues, they are also most likely charged and therefore not idea species for adduction. 
Figure 43 below demonstrates the outer Lys (red) and His (pink) residues for HSA.
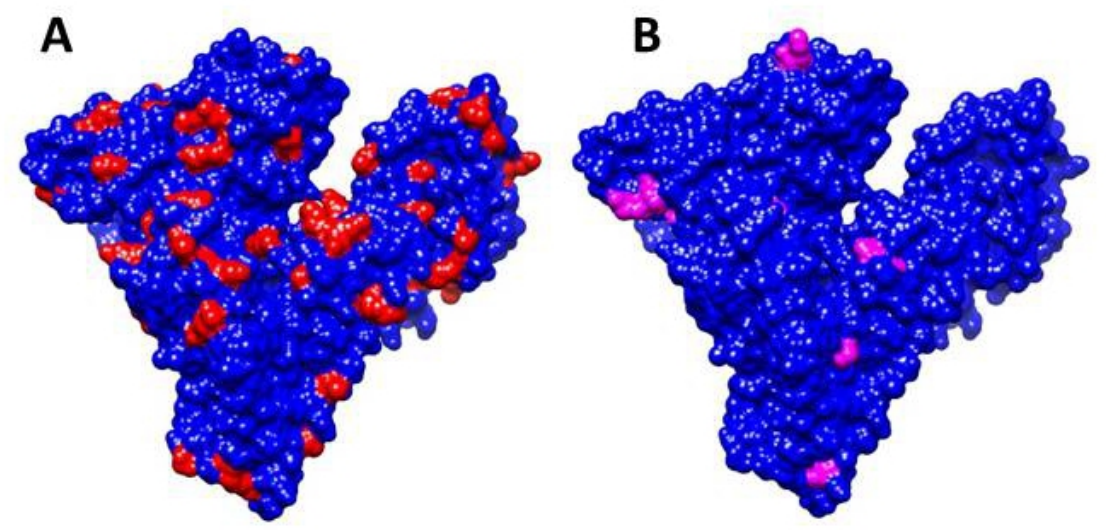

Figure 43: Space-filled model of HSA, with Highlighted Lys (A) and His (B) residues. HSA is shown in blue, the red residues represent the outer Lys residues, and the pink residues represent outer His residues.

Immediately evident by this figure is the larger number of Lys residues (red) present on the surface of HSA, when compared to surface His residues (pink). Unlike Hb, adduction of HN-2 and HN-3 at two Lys residues was identified reproducibly. This suggests that, despite the higher base $\mathrm{pK}_{\mathrm{a}}$ value of Lys, local electronic factors within HSA may allow for these sites to be adducted by $\mathrm{HN}-2$ and $\mathrm{HN}-3$. Additional work should be performed in which the local electronic environment for these sites are analyzed further (particularly for the Lys residues on HSA) for theoretical explanation of site specific modification by $\mathrm{HN}-2$ and $\mathrm{HN}-3$.

The focus of this research was to identify novel and specific adduction sites of HN-2 and HN-3 to Lys and His residues as additional exposure biomarkers that may be more applicable than those previously identified for these compounds. This was indeed accomplished with the identification of reproducible Lys and His adduction by both HN-2 and HN-3. However, the results obtained for this task did not reveal specific modification of the known reactive Cys residues on $\mathrm{Hb}$ (Cys-93ß) or HSA (Cys-34). Several hypotheses can be proposed to account for why such adducts were not identified in the present study. 
For $\mathrm{Hb}(\mathrm{Cys}-93 \beta)$, no formal identification of $\mathrm{HN}-2$ or HN-3 modification has been published. This residue is considered highly reactive due to its proximity to His-92 $\beta$, which is partially responsible for heme incorporation into the $\mathrm{Hb}$ tertiary structure, ${ }^{55}$ therefore affecting the Cys local environment so that it is more susceptible for adduction (more nucleophilic). However, this residue is also present in a hydrophobic pocket of the protein, which would be difficult for a hydrophilic or charged intermediate (such as reactive $\mathrm{HN}-2$ and $\mathrm{HN}-3$ aziridinium intermediates) to access. This possibility is, in part, supported by the data obtained in the present task, where all identified HN-2 and HN-3 modifications were localized on the surface of the protein. In addition, HN-2 and HN-3 are known to hydrolyze quickly in aqueous environment - as such, it may be that these reactive species would not be present long enough to adduct to Cys-93 $\beta$, in view of its low accessibility.

For HSA (Cys-34), it was expected that this residue would be modified by HN-2 and HN3, based on work from previous researchers. ${ }^{93,133}$ However, it is important to compare the methodologies utilized in these publications to those employed in the present study. Noort et al. identified HN-2 Cys-34 adducts following Pronase digestion of HSA based on previous work done with identification of Cys-34 adducts by sulfur mustard. ${ }^{80}$ Pronase is a proteolytic enzyme that is relatively non-specific in terms of protein cleavage. ${ }^{100}$ A second method utilized in that work was $\mathrm{HCl}$ digestion, a technique used to break a protein into many small peptides. Both of these approaches are quite useful in producing small, manageable peptides for analysis; however, they are not optimal when trying to identify novel sites of adduction (as was the goal of this work). For such an application, a proteolytic enzyme is needed that has a high level of specificity so as to easily predict peptides formed from a protein digest, which can then be used for prediction of adduction sites. Trypsin was intentionally utilized for this project for its specificity; however, the tryptic fragment produced containing the Cys-34 residue is a large-mass peptide (21 amino acids) that may be lost or difficult to detect. 
In addition, Noort and colleagues were able to detect the HD Cys modification by means of radiolabeled sulfur mustard and isolation of an LC fraction, later identified as a Cys-modified tri-peptide. This result was further confirmed through comparison to a synthetic standard once the tri-peptide was characterized. Identification of the HN Cys-34 adduct was assumed to occur on the same tri-peptide following pronase digestion, which was indeed the case. Consequently, the sensitivity for detection of the Cys-34 specific modification was likely to be higher in the previous work than in the present study.

Finally, because of the variety of amino acids present on the expected tryptic fragment containing Cys-34, it is possible that multiple residues within the peptide may have been modified, or that $\mathrm{HN}-2 / \mathrm{HN}-3$ may be forming cross-links on the peptide. The expected tryptic fragment containing Cys-34 on HSA is ALVLIAFAQYLQQCPFEDHVK. While the Cys is an expected adduction site, the His may also be available for adduction, in addition to other nucleophilic residues such as Tyr (T), Glu (E), and Asp (D). While a His residue would be allowed in the BioConfirm Data analysis, a third adduction to Tyr, Glu, or Asp would cause the peptide to be missed by BioConfirm using the parameters listed in Appendix 5 (potential adduction by $\mathrm{HN}-2 / \mathrm{HN}-3$ to other amino acids is further discussed in Section 4.5). Furthermore, if a cross-link exists between any of these sites, this modification would also not have been determined and therefore not programmed into BioConfirm to recognize. Recent work regarding nitrogen mustards has identified their ability to cross-link in proteins; ${ }^{137}$ it is possible that, given the surrounding amino acids, a cross-link exists that is currently unknown. Further work, including confirmation of adduction to additional amino acids, further optimization of LC-MSMS parameters, and investigation of $\mathrm{HN}-2$ and $\mathrm{HN}-3$ crosslinking, would therefore be required to identify the Cys adduct given the conditions used for this work. 


\subsection{Whole Blood Incubation and In Vitro Protein Adduction}

Finally, a proof-of-concept study was performed in which whole blood was incubated with $\mathrm{HN}-2$ and $\mathrm{HN}-3$ to determine whether the identified $\mathrm{Hb}$ and HSA adducts would form in a simulated in vivo environment. Prior to $\mathrm{HN}$ incubations, the precipitation extraction procedures

for $\mathrm{Hb}$ and $\mathrm{HSA}$ were tested via $\mathrm{C}_{8}$ analysis for general determination of extraction purity. Figure 44 compares the $\mathrm{Hb}$ extracted from whole blood to a standard created from lyophilized protein purchased at high purity. 


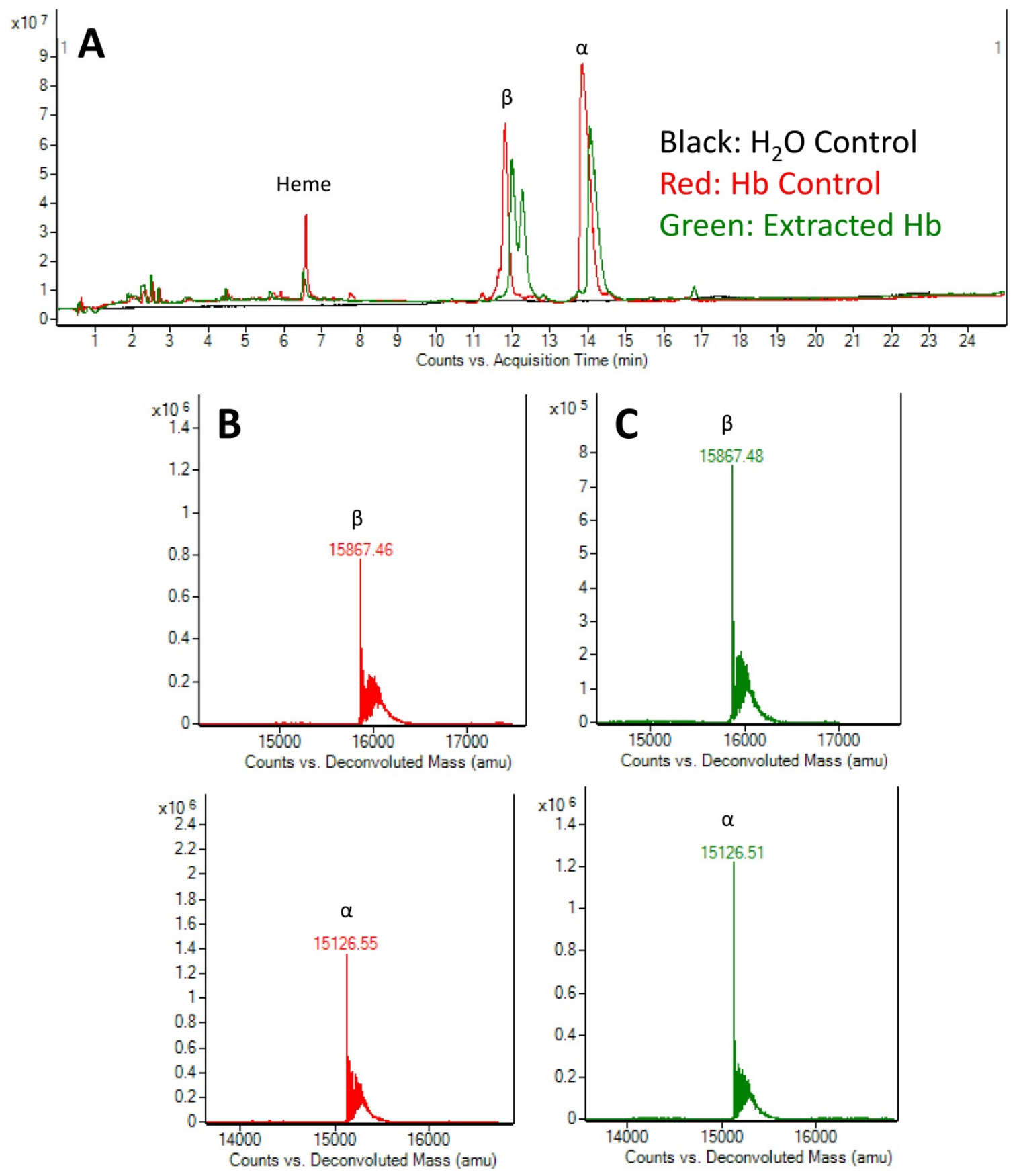

Figure 44: $\mathrm{C}_{8}$ reversed phase $\mathrm{LC}$ analysis of extracted $\mathrm{Hb}$ (green) as compared to a standard protein (red). A: TIC; B: Deconvolution results for the control $\alpha$ and $\beta$ peaks; $C$ : deconvolution results for extracted $\alpha$ and $\beta$ peaks, demonstrating successful extraction and identification of $\mathrm{Hb}$ from whole blood.

The extraction method for $\mathrm{Hb}$ provided a clean extraction and was therefore considered acceptable for the whole blood incubation studies. The slight variation in RT between extracted 
and purified proteins was within the previously set cutoff of $\pm 0.3 \mathrm{~min}$ and therefore considered acceptable. Additionally, the split peak observed for the $\beta$ subunit is most likely a variant as a results of $\mathrm{Hb}$ extraction from a pooled blood sample.

Figure 45 compares the HSA extracted from whole blood to a standard created using lyophilized HSA purchased at high purity. As with $\mathrm{Hb}$, the HSA precipitation extraction provided clean results that were deemed acceptable for this work. As mentioned previously for $\mathrm{Hb}$, the deconvoluted mass difference is most likely a result of variability (genetic or otherwise) between the pure standard and the extracted blood sample, in addition to the complexity of MS data obtained for deconvolution.

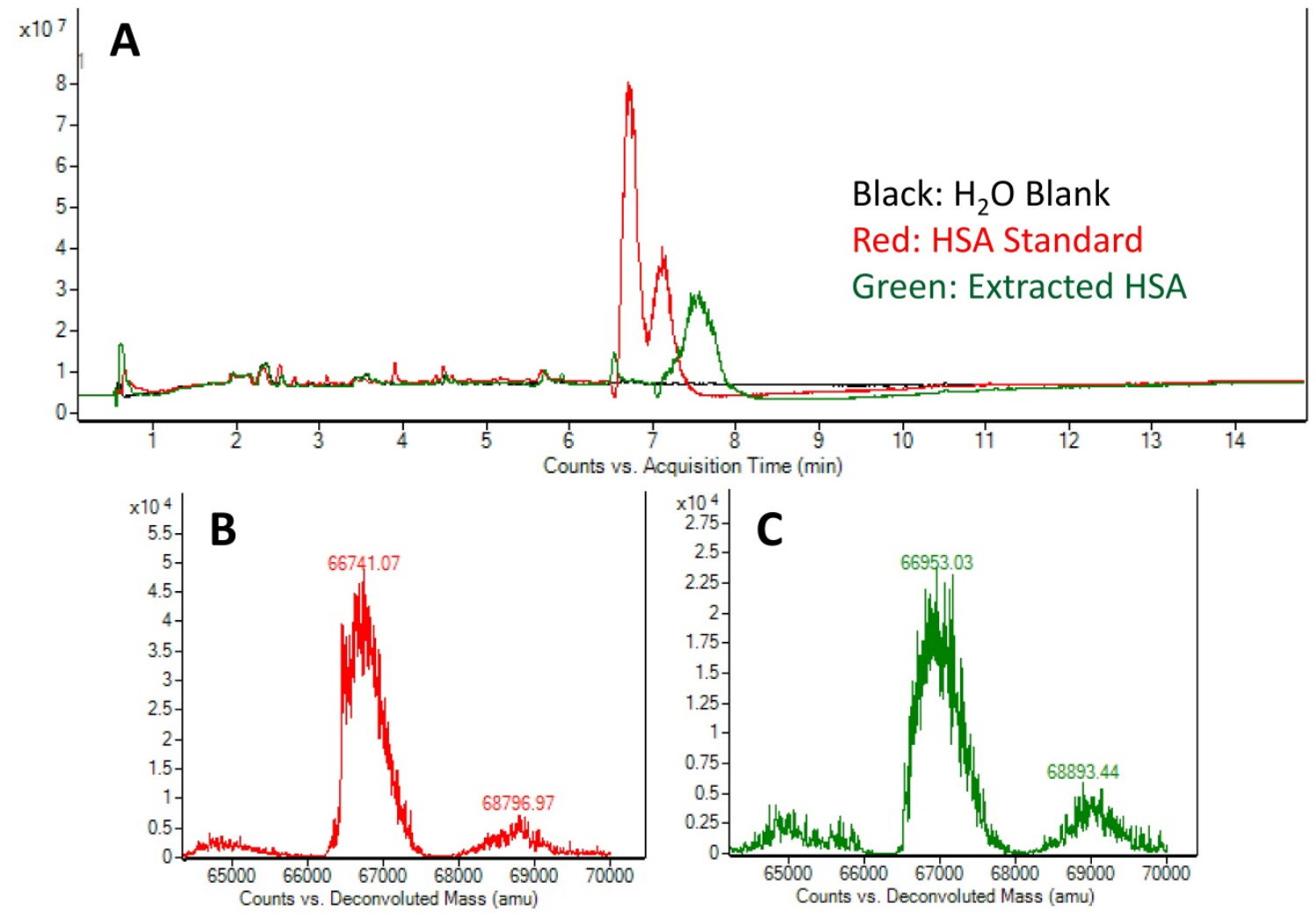

Figure 45: $\mathrm{C}_{8}$ reversed phase $\mathrm{LC}$ analysis of extracted HSA (green) as compared to a protein standard (red). A: TIC; B: Deconvolution results for HSA standard; C: Deconvolution results for extracted HSA, showing successful identification and extraction of HSA from whole blood. 
There are several different methods in which proteins may be extracted from whole blood. The precipitation reactions selected for this work were chosen due to their use in previous adduct studies; however, newer methods have been introduced that may also provide acceptable recoveries for the proteins of interest. Affinity chromatography has been increasingly utilized for the extraction of HSA from serum, with excellent results. ${ }^{138}$ During initial determinations of extraction methods suitable for this work, affinity columns were tested for the extraction of adducted HSA, and demonstrated similar results as seen with the precipitation extraction of HSA. The precipitation reaction was ultimately chosen as the method of choice due to its use of fewer reagents, cost-effectiveness, and time-saving protocol. While the results obtained for this particular experiment were comparable between the two protocols, it is possible that other adduction studies may produce adduct structures that may hinder the recognition of HSA in the affinity column due to the modification. Care should therefore be taken when extracting modified HSA in such cases.

Whole blood was incubated with HN-2 and HN-3 and the HSA was separated from the plasma, while the $\mathrm{Hb}$ was separated from the erythrocytes. Extracted proteins were then digested and analyzed via LC-Q-TOF-MS-MS. Analysis of triplicate blood samples for HN-2 and HN-3 revealed the presence of the previously identified specific adducts, matched by modification description and retention time. These in vitro incubations demonstrate the stability of the adducts even after precipitation extraction of $\mathrm{Hb}$ and $\mathrm{HSA}$, which further strengthens their potential use as exposure biomarkers to $\mathrm{HN}-2$ and $\mathrm{HN}-3$.

Additional validation of $\mathrm{Hb}$ and $\mathrm{HSA}$ adducts identified in this study as potential specific biomarkers of $\mathrm{HN}$ exposure would benefit from in vivo studies in animal models. However, care must be taken in extrapolating the results of adduction studies in animal models to predict specificity in humans. Typically, toxicity studies are done using a rat model. ${ }^{139}$ However, despite the large overlap between human and rat $\mathrm{Hb}$ and serum albumin (SA) sequences, some of the 
adduction sites identified in the present study do not exist in rat $\mathrm{Hb}$ or SA. Figure 46 shows a comparison between the $\alpha$ and $\beta$ chains of $\mathrm{Hb}$ in human and rat. Adduction to His-50 $\alpha$ could not occur in rat $\mathrm{Hb}$, as this site is not conserved (His-50a is replaced with a Pro at this site).

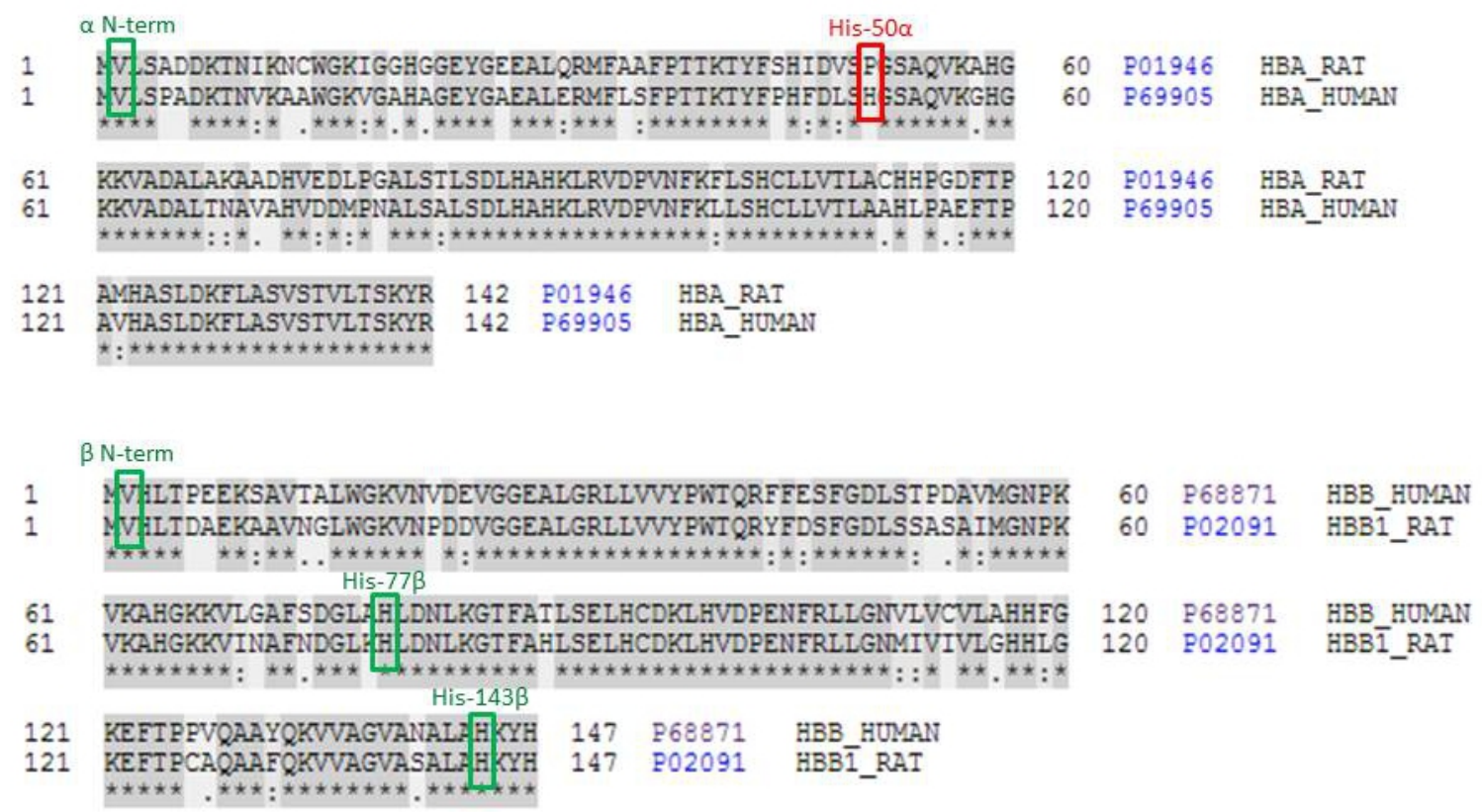

Figure 46: Comparison of the $\mathrm{Hb}$ amino acid sequences of human and rat. Sites highlighted in green are conserved within species, while the sites highlighted in red are not conserved between species.

Figure 47 shows a comparison of the SA sequences for human and rat. While both Lys residues of interest are conserved in the rat, the N-terminus is a Glu (versus Asp) in rat. Additionally, His-367 is replaced with a Pro. 


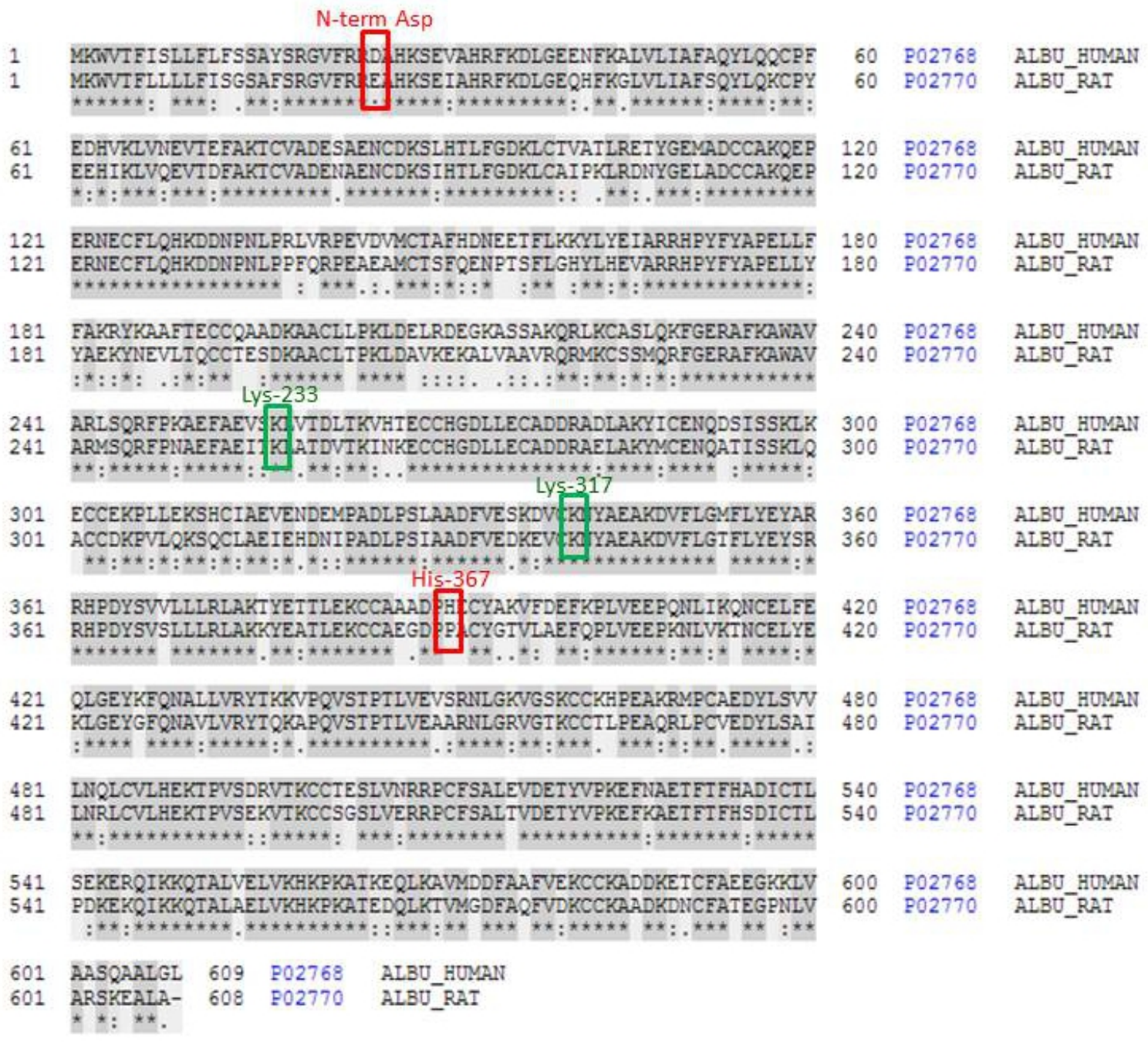

Figure 47: Comparison of SA amino acid sequences of human and rat. Sites highlighted in green are conserved among species, whereas sites highlighted in red are not conserved between species.

These differences emphasize the importance in using human proteins for the development of specific adduct-based exposure biomarkers for this class of compounds. 


\subsection{In Vitro Metabolism of Nitrogen Mustard Therapeutic Compounds}

The previously obtained results provided a strong indication that the hydrolyzed adducts $(\mathrm{HN}-2 \mathrm{OH}$ and $\mathrm{HN}-3 \mathrm{OH})$ were the more relevant adducts in terms of biological protein adductions and long-term exposure biomarkers; therefore, for the metabolic studies, it was of interest to determine whether commonly encountered therapeutic drugs containing the nitrogen mustard moiety (cyclophosphamide, chlorambucil, and melphalan) would, after metabolism, produce the same covalently bound adducts as HN-2 or HN-3. The structures of these compounds may be seen in Figure 48. As the goal of this project is to identify not only novel, but specific biomarkers of exposure, it is of high importance to assess whether these drugs are also capable of forming the same adducts on Cys, Lys, or His.

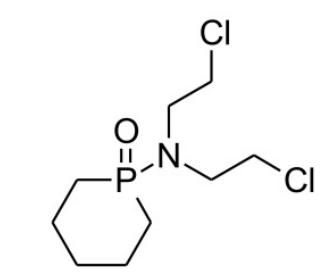

Cyclophosphamide

(CP)<smiles>O=C(O)CCCc1ccc(N(CCCl)CCCl)cc1</smiles>

Chlorambucil

(CB)<smiles>N[C@@H](Cc1ccc(N(CCCl)CCCl)cc1)C(=O)O</smiles>

Melphalan

(MP)

Figure 48: Structures of commonly used nitrogen-mustard containing therapeutic agents studied in the present investigation.

As detailed in the methods section, a SIM method was utilized for this experiment. While it is known that these therapeutic compounds are capable of adducting to nitrogen in DNA (as this is their mode of action as antineoplastic drugs), ${ }^{24,46,114}$ the focus here is to ensure that protein adduction by these drugs would not produce the same adduct structures as previously identified for HN-2 and HN-3. Therefore, SIM methods were utilized as a rapid comparison for adducts of interest, as the molecular weights and $\mathrm{RT}$ of $\mathrm{HN}$-adducted peptides were identified and characterized in previous studies. In addition, controls were utilized in which $\mathrm{HN}-2$ and $\mathrm{HN}-3$ 
were added to the metabolic systems. It was been shown in the present and previous work that these compounds will adduct to model peptides without the need for metabolic assays; therefore, it would be reasonable to expect that previously identified adducts would still be generated in these control assays.

\subsubsection{Cyclophosphamide}

The previously reported metabolic pathway of CP may be found in Appendix 1. The reactive species formed after metabolism is nornitrogen mustard, which contains the same 2chloroethyl backbone as $\mathrm{HN}-2$ and $\mathrm{HN}-3$; however, the third group on the central nitrogen is a hydrogen vs. a methyl (HN-2) or 2-chloroethyl (HN-3). As such, it is not expected that metabolized CP will form the same adduction species with the model peptides as is seen with HN-2 or HN-3.

MS analysis of the control samples metabolized by S9 fraction demonstrated expected peaks corresponding to previously identified hydroxylated adducts. These adducts were confirmed via full-scan MS analysis of the assay samples and RT matching from previously run samples. When the control samples were compared to the respective SIM trace obtained in triplicate CP samples, there were no overlapping peaks present, meaning that S9-metabolized CP does not produce the same hydroxyl adducts with Cys, Lys, or His as HN-2 or HN-3. Representative results of S9-metabolized CP and corresponding control experiments may be seen in Figure 49. 

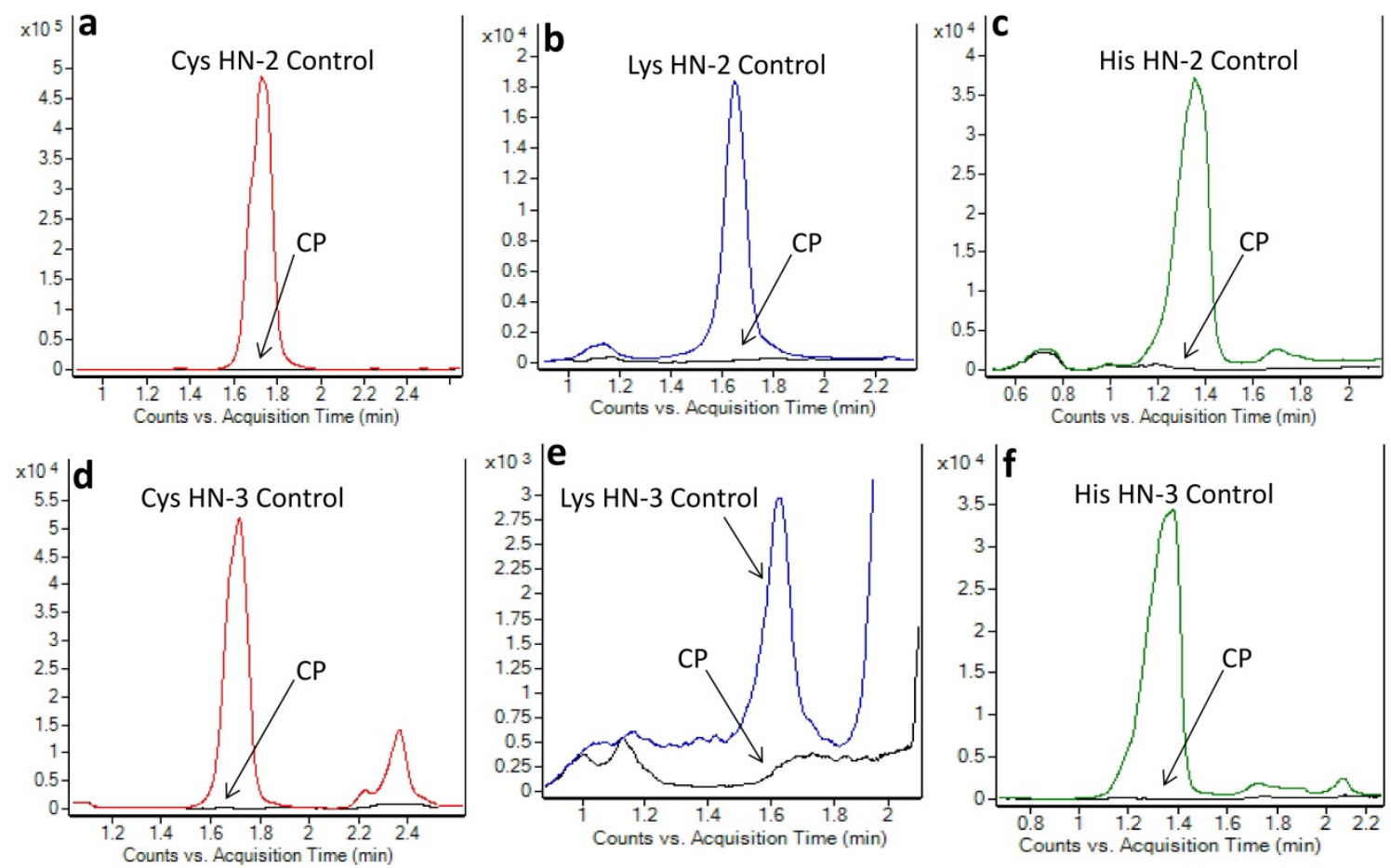

Figure 49: S9-metabolism of CP, as compared to controls (peptides incubated with $\mathrm{HN}-2$ and $\mathrm{HN}-3$ ) samples. HN-2 (a-c) and HN-3 (d-f) controls were present in respective samples, but no overlap was seen with CP-containing triplicates [black lines].

HLM-metabolized CP samples yielded similar results to the S9-metabolized samples. All control samples yielded peaks corresponding to previously identified hydroxyl adducts of $\mathrm{HN}-2$ and HN-3 with the model peptides, confirmed via full scan MS analysis and RT matching. When compared to CP-containing samples, no overlap was observed at the same RT as the control samples. Based on these results, HLM-metabolized CP does not produce the same adducts with Cys, Lys, and His as HN-2 or HN-3 (Figure 50). 

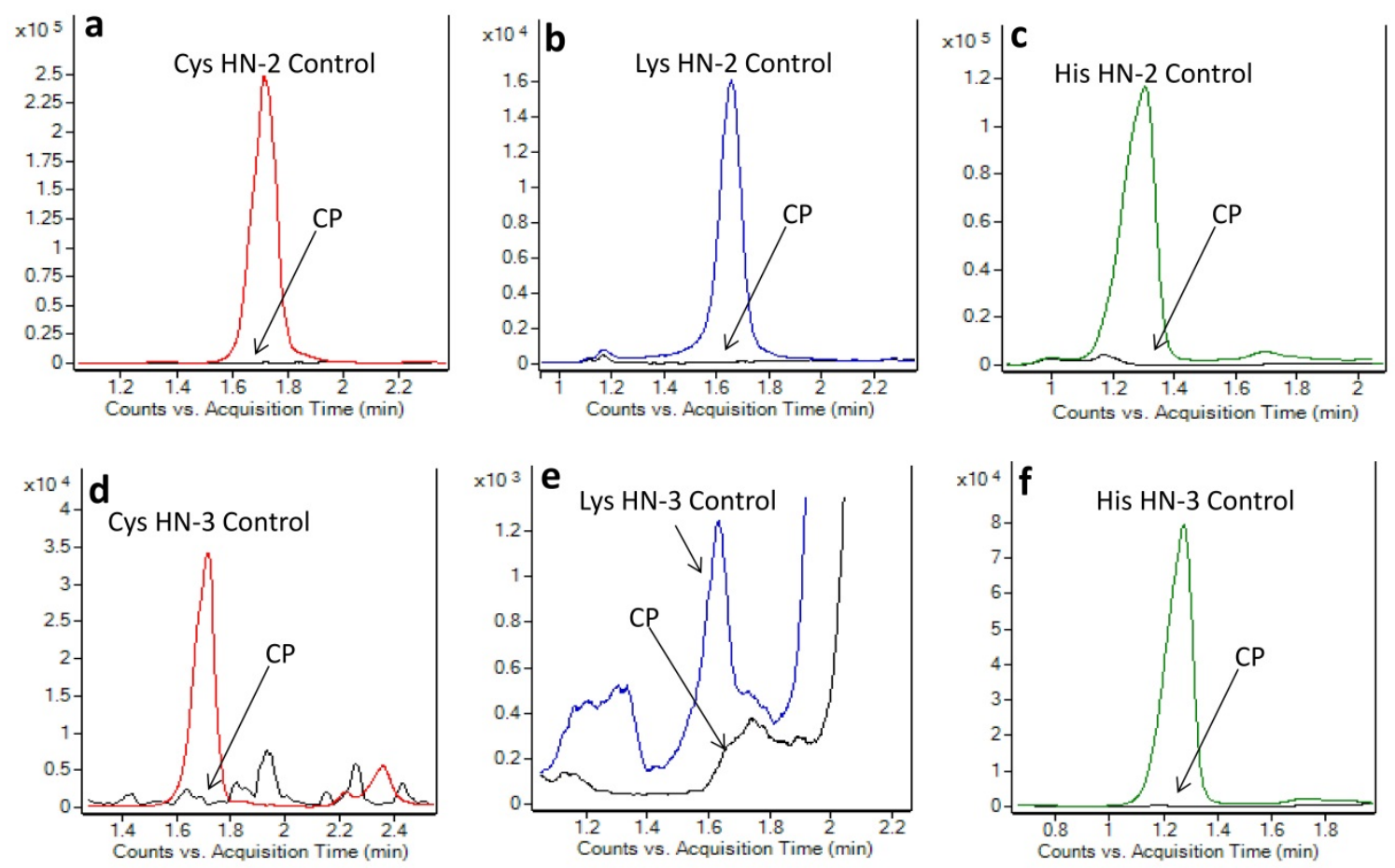

Figure 50: HLM-metabolized CP [black trace] as compared to $\mathrm{HN}-2$ (a-c) and $\mathrm{HN}-3$ (d-f) controls.

\subsubsection{Chlorambucil}

The previously documented metabolism for CB may be found in Appendix 2. The primary metabolite of $\mathrm{CB}$ is phenylacetic acid mustard, formed via $\beta$-oxidation of the parent compound. No known metabolites of $\mathrm{CB}$ have been determined in which the phenylacetic acid group is not attached.

Analysis of the control samples after S9 metabolism yielded similar results as seen with cyclophosphamide. SIM peaks corresponding to the $\mathrm{HN}-2$ and $\mathrm{HN}-3$ hydroxylated adducts were correctly identified based on full scan MS analysis and RT matching from previously run samples. When compared to CB-containing samples, there were no peaks that matched to the control samples (Figure 51). Therefore, based on these results, S9-metabolized CB does not form the same hydroxylated adducts as HN-2 or HN-3. 

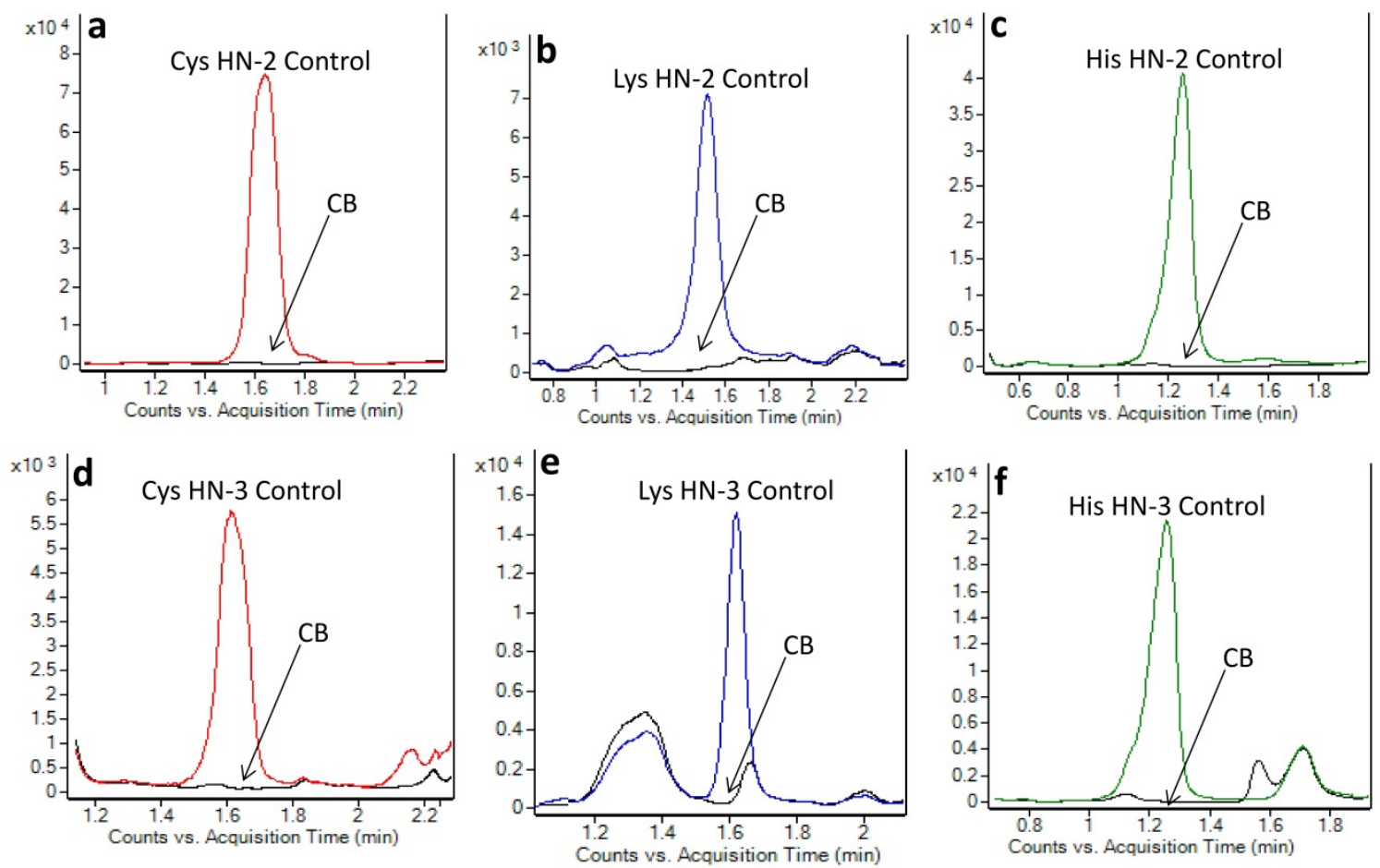

Figure 51: S9 metabolized CB [black trace] as compared to control samples $\mathrm{HN}-2$ (a-c) and $\mathrm{HN}-3$ (df).

Analysis of the HLM-metabolized samples yielded similar results as seen with S9

metabolism. Peaks corresponding to the expected HN-2 and HN-3 hydroxylated adducts were present in the control samples, confirmed through full scan MS analysis of the controls and RT matching. For all three peptides, there was no overlap observed in the CB-containing samples as compared to the controls (Figure 52). These results suggest that, when metabolized with HLM, CB does not produce the same adducts as HN-2 or HN-3 with Cys, Lys, or His. 

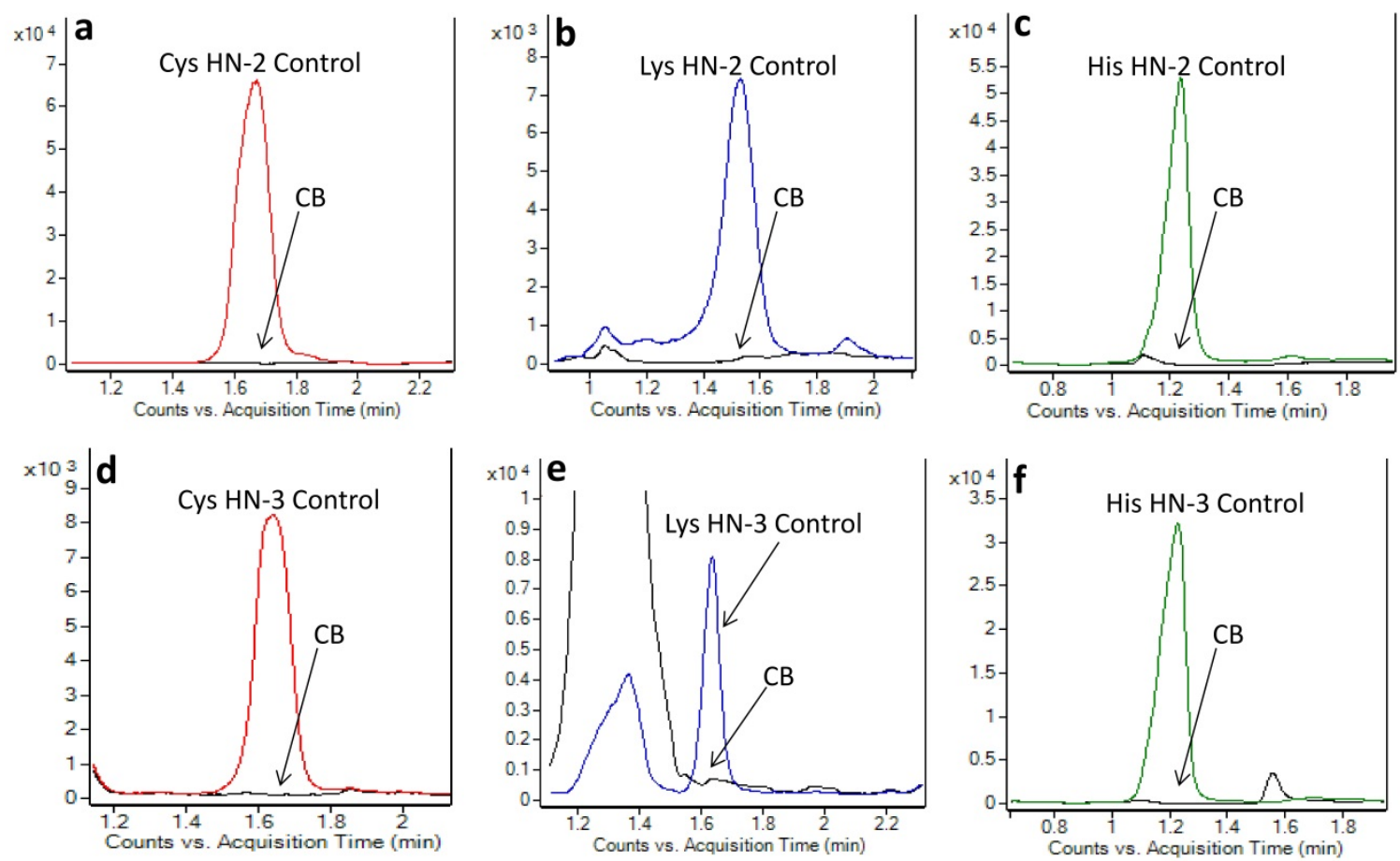

Figure 52: HLM metabolized CB [black trace] as compared to control samples HN-2 (a-c) and HN-3 (d-f).

\subsubsection{Melphalan}

The known metabolic pathway of MP can be found in Appendix 3. The major known metabolite for MP is hydroxy-MP; no know metabolites of MP have been identified that do not include the benzyl side chain.

Controls for the S9-metabolized samples yielded expected peaks corresponding to the hydroxylated $\mathrm{HN}-2$ and $\mathrm{HN}-3$ adducts. As with previous controls, these were confirmed via full scan analysis of the metabolism samples and RT matching from previous results. When samples containing MP were compared to these controls, there were no observed overlaps for the adduct peaks. These results demonstrate that S9-metabolized MP will not produce the same adducts as HN-2 or HN-3 with Cys, Lys, or His residues (Figure 53). 

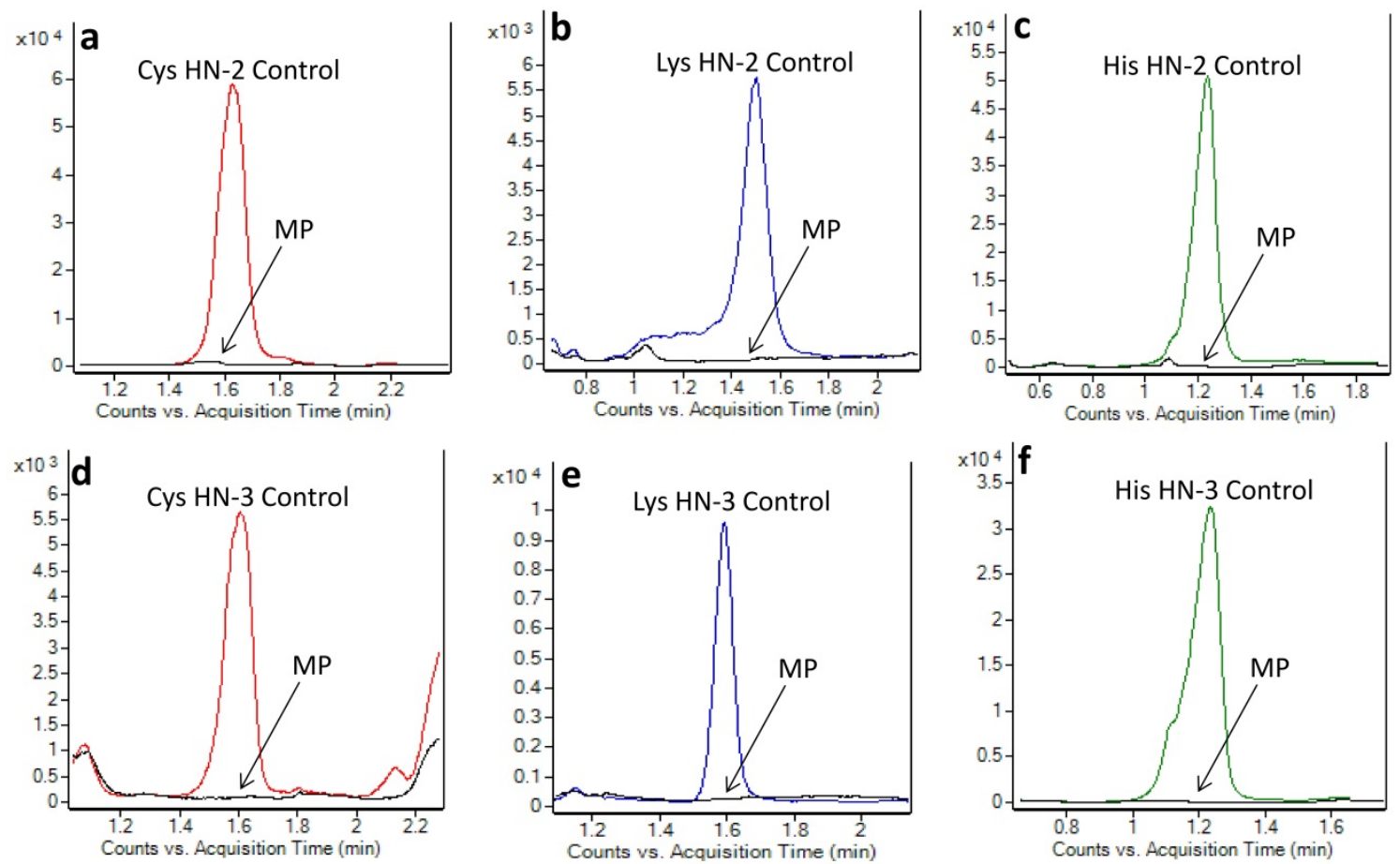

Figure 53: S9 metabolized MP [black trace] as compared to control samples HN-2 (a-c) and HN-3 (df).

The results obtained from HLM-metabolized samples are similar to what was obtained in the S9 fraction. Control samples yielded expected SIM peaks corresponding to hydroxylated HN2 and HN-3 adducts, confirmed through full scan analysis of the metabolized samples and RT matching from previous results. Metabolized samples containing MP were compared to these control samples, and no overlap was observed in all six conditions. Based on these results, HLMmetabolized MP will not form the same adducts as HN-2 or HN-3 with Cys, Lys, or His (Figure 54). 

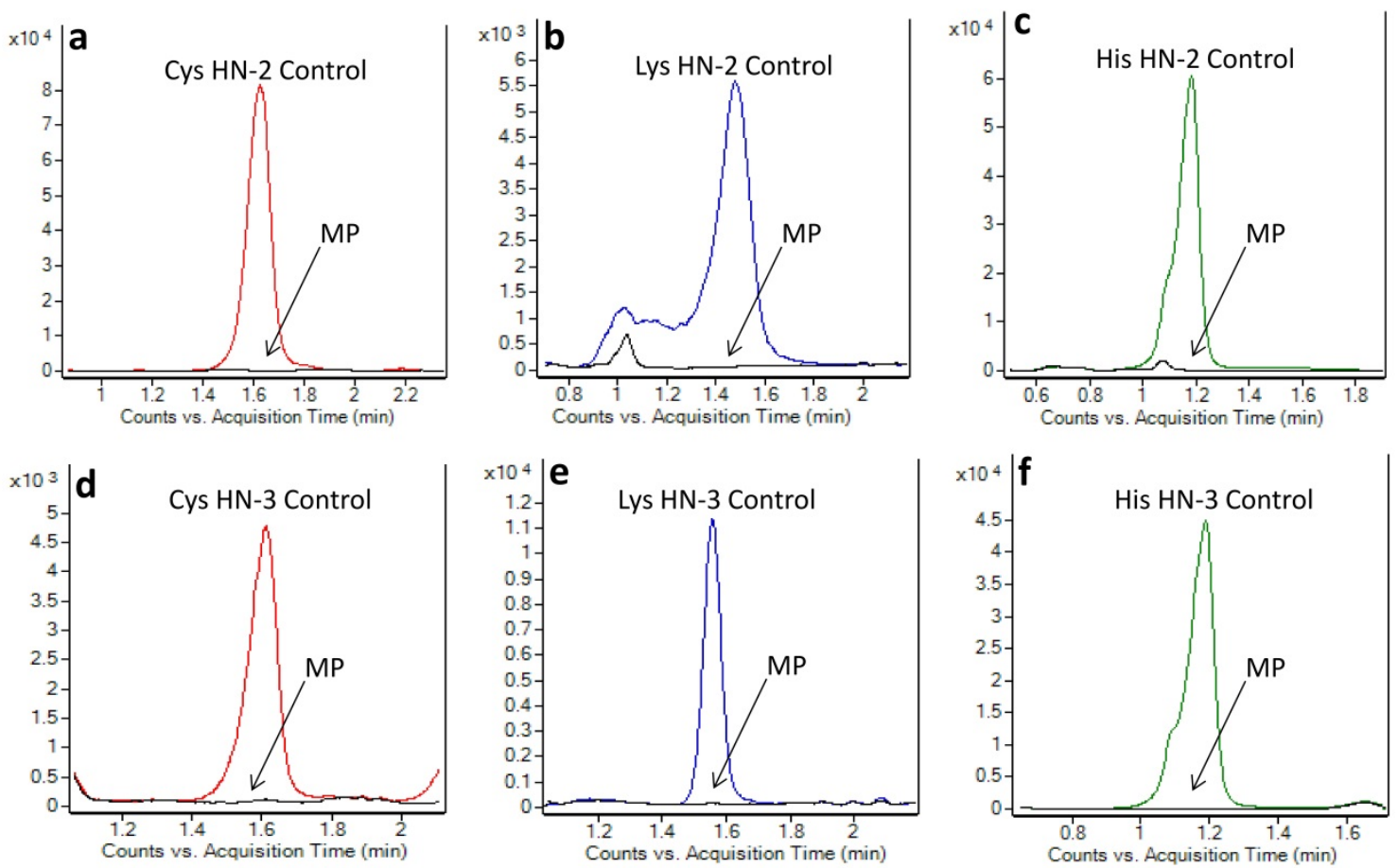

Figure 54: HLM metabolized MP [black trace] as compared to control samples HN-2 (a-c) and HN-3 (d-f).

\subsubsection{Discussion}

It is of importance to note that this study was not intended to characterize specific adducts formed by reactive species present after metabolism of these drugs (nornitrogen mustard, etc.). In fact, based on the known reactivity of nitrogen mustards in general, it is likely that such reactive metabolites did form adducts with the nucleophilic residues present on these peptides. However, the metabolic assays conducted under this task successfully demonstrated that, despite some structural similarities to the CWA HN-2 and $\mathrm{HN}-3$, the three therapeutic drugs tested (Cyclophosphamide, Chlorambucil, and Melphalan) did not produce the same specific adduct structures as observed for $\mathrm{HN}-2$ or $\mathrm{HN}-3$ at each of the three nucleophilic sites of interest. This is an important set of results, as it indicates that prior or concurrent exposure to such drugs would not result in false positive detection of $\mathrm{HN}$ exposure based on specific adduct detection. Nevertheless, it is possible that such drugs could adduct the same sites on $\mathrm{Hb}$ and HSA as HN-2 
and $\mathrm{HN}-3$ to produce structurally distinct adducts. This possibility could be evaluated in future research. 


\subsection{Quantum Mechanical Calculations}

\subsubsection{Electrophilicity of Nitrogen Mustards and Related Intermediates}

Table 8 shows the calculated chemical potentials $(\mu)$ and hardness values $(\eta)$ (as calculated from Equations 2 and 3) for $\mathrm{HN}-2$ and its related intermediates based on the HOMOLUMO energies obtained from Gaussian. The compounds are listed in order from most electrophilic (more negative chemical potential) to least electrophilic. In addition, the table also includes data for a comparative compound, acrylamide, which is a known neurotoxicant and has previously been classified as a soft electrophile, ${ }^{62}$ for comparison purposes to the obtained $\mathrm{HN}-2$ data.

\begin{tabular}{|c|c|c|c|}
\hline Compound Name & Structure & $\mu(\mathrm{eV})$ & $\eta(e V)$ \\
\hline $\mathrm{HN}-2 \mathrm{Az} \mathrm{Cl}$ & & -7.97 & 3.95 \\
\hline $\mathrm{HN}-2 \mathrm{Az} \mathrm{OH}$ & & -7.34 & 4.12 \\
\hline $\mathrm{HN}-2$ & & -2.97 & 3.42 \\
\hline $\mathrm{HN}-2 \mathrm{OH} \mathrm{Cl}$ & & -2.67 & 3.41 \\
\hline $\mathrm{HN}-2 \mathrm{OH} \mathrm{OH}$ & & -1.90 & 3.83 \\
\hline Acrylamide & & -3.80 & 2.96 \\
\hline
\end{tabular}

Table 8: Calculated chemical potentials $(\mu)$ and hardness values $(\eta)$ for $\mathrm{HN}-2$ and related intermediates, as calculated by Equations (2) and (1), respectively. Acrylamide is used as a reference compound for comparison purposes.

These results demonstrated that the Az ion containing one chlorine still attached is the most electrophilic species of the $\mathrm{HN}-2$ intermediates, and therefore most likely to bind to nucleophilic species as compared to other $\mathrm{HN}-2$ intermediates. This adduct may then undergo 
hydrolysis in aqueous environments to yield the stable hydroxylated adducts observed in this work. It is also important to note that the hydroxylated Az is also a strong electrophile, as shown in Table 8. Adduction may therefore follow a secondary path in which the $\mathrm{HN}-2 \mathrm{Az} \mathrm{Cl}$ intermediate first hydrolyzes, followed by adduction. Current research is still unclear as to which mechanism occurs in aqueous conditions, or whether both mechanisms occur simultaneously. Regardless, these calculations not only support previous hypotheses regarding the formation of these $\mathrm{Az}$ ions as the electrophilic species responsible for protein and DNA binding, they also suggest that multiple pathways are possible as both Az species are comparably electrophilic. The final compound formed from $\mathrm{HN}-2$ hydrolysis $(\mathrm{HN}-2 \mathrm{OH} \mathrm{OH}$, or $\mathrm{N}$-methyldiethanolamine) was determined to have the highest value for chemical potential, and therefore the least nucleophilic character of the HN-2 intermediates. This finding is supported in experimental data, in which model peptides incubated with $\mathrm{HN}-2 \mathrm{OH} \mathrm{OH}$ for $24 \mathrm{~h}$ at physiological conditions did not yield adducts on Cys, Lys, or His.

In terms of chemical hardness calculations (calculated via Equation 1), the larger the value of $\eta$, the "harder" the compound is expected to be. Hard electrophiles are typically described as containing a localized charge density around a central electrophilic center. ${ }^{62}$ In the case of nitrogen mustards, the positively charged nitrogen in the center of the molecule would house the charge density for the molecule. The calculated hardness values for $\mathrm{HN}-2$ and its intermediates indicate that these compounds may be considered as hard electrophiles. While there is no distinct cutoff in hardness values that can be used to distinguish between hard and soft compounds, the calculated values for compounds typically considered to be soft may be used as relative comparisons. As previously mentioned, acrylamide is a selective neurotoxicant that has been described as a soft electrophilic species. The hardness values for the calculated HN-2 intermediates are higher than that of acrylamide, again suggesting that these compounds should be classified as hard or moderately hard electrophilic species. As a consequence, their reactivity 
towards nucleophilic species should be expected to occur not only on soft nucleophiles such as thiol, but on other harder species such as Lys and His.

Table 9 demonstrates the chemical potential values $(\mu)$ and hardness values $(\eta)$ for HN-3 and its related intermediates, as calculated by Equations 1 and 2. As with $\mathrm{HN}-2$, acrylamide is listed at the bottom of the table as comparison. Compounds are ranked by the most negative chemical potential (most electrophilic species) to those with the least negative chemical potential (least electrophilic species).

As with HN-2, the HN-3 Az species demonstrate the most negative chemical potential values, and therefore are considered the most electrophilic species. This again lends credibility to the hypothesized formation of the Az ion being the intermediate that binds to nucleophilic species such as proteins and DNA. The similarities seen with the chemical potential of these three Az species also suggest that a variety of mechanisms are available for adduction. While the doublechlorinated adduct would be considered the most electrophilic species, the bulk of two chlorines present on the molecule may prevent adduction in the case of a steric-hindered nucleophile. For this reason, the hydrolyzed species (whether with a loss of one chlorine or both) may be a more favored adducting species as they are less bulky and similarly reactive. As with HN-2, the terminal intermediate of $\mathrm{HN}-3$ in which all three chlorines have undergone hydroxylation (HN-3 $\mathrm{OH} \mathrm{OH} \mathrm{OH}$, or triethanolamine) is the compound with the lowest chemical potential, and therefore the intermediate least likely to bind to nucleophilic sites. This is also supported by experimental data in which adducts to Cys, Lys, and His did not form at physiological conditions when incubated with triethanolamine. 


\begin{tabular}{|c|c|c|c|}
\hline Compound & Structure & $\mu(\mathrm{eV})$ & $\eta(\mathrm{eV})$ \\
\hline $\mathrm{HN}-3 \mathrm{Az} \mathrm{Cl} \mathrm{Cl}$ & & -8.09 & 3.79 \\
\hline $\mathrm{HN}-3 \mathrm{Az} \mathrm{OH} \mathrm{Cl}$ & & -7.69 & 3.78 \\
\hline $\mathrm{HN}-3 \mathrm{Az} \mathrm{OH} \mathrm{OH}$ & & -7.31 & 4.00 \\
\hline $\mathrm{HN}-3$ & & -3.33 & 3.37 \\
\hline $\mathrm{HN}-3 \mathrm{OH} \mathrm{Cl} \mathrm{Cl}$ & & -3.01 & 3.38 \\
\hline $\mathrm{HN}-3 \mathrm{OH} \mathrm{OH} \mathrm{Cl}$ & & -2.72 & 3.43 \\
\hline $\mathrm{HN}-3 \mathrm{OH} \mathrm{OH} \mathrm{OH}$ & & -2.10 & 3.75 \\
\hline Acrylamide & & -3.80 & 2.96 \\
\hline
\end{tabular}

Table 9: Calculated chemical potentials $(\mu)$ and hardness values $(\eta)$ for $\mathrm{HN}-3$ and related intermediates, as calculated by Equations (2) and (1), respectively. Acrylamide is included in the table for comparison purposes.

When the hardness values for HN-3 intermediates are compared to a soft electrophile (acrylamide), it is evident that these compounds are harder electrophiles. This may explain why HN-3 binds not only to the soft thiol on Cys, but also the moderately hard nucleophilic centers of Lys and His. The hardness values of HN-2 and HN-3 (and corresponding intermediates) may also 
be compared with each other. The values for the Az species are all comparable, suggesting that HN-2 and HN-3 should interact with a similar set of nucleophiles, a prediction supported by the results obtained for both peptide and protein adduction studies. The slight differences in adduction between $\mathrm{HN}-2$ and $\mathrm{HN}-3$ may not necessarily be electronically determined, as suggested by the similar chemical potential values obtained for $\mathrm{HN}-2$ and $\mathrm{HN}-3$ Az intermediates, but instead based on steric limitations of $\mathrm{HN}-3$ over $\mathrm{HN}-2$ as previously proposed.

\subsubsection{Nucleophilicity of Amino Acids}

Table 10 shows the calculated chemical potential and hardness values for the three amino acids that were the focus of this work (Cys, Lys, and His). Values were calculated for both for the neutral and deprotonated amino acids. Compounds are ranked from highest chemical potential (and therefore the better nucleophile) to lowest chemical potential. As expected, the cysteine thiolate is calculated to be the most nucleophilic species studied. In addition, the calculated chemical potential values for neutral Lys and His are very similar. It is also important to note that the hardness values of Lys and His are higher than the value for Cys, albeit not extremely so. This supports the classification of these two amino acids as "moderately hard" nucleophiles. This intermediate value supports the idea that while Lys and His contain nitrogen as the nucleophilic atom, they may be susceptible to attack by both soft and hard electrophiles. 


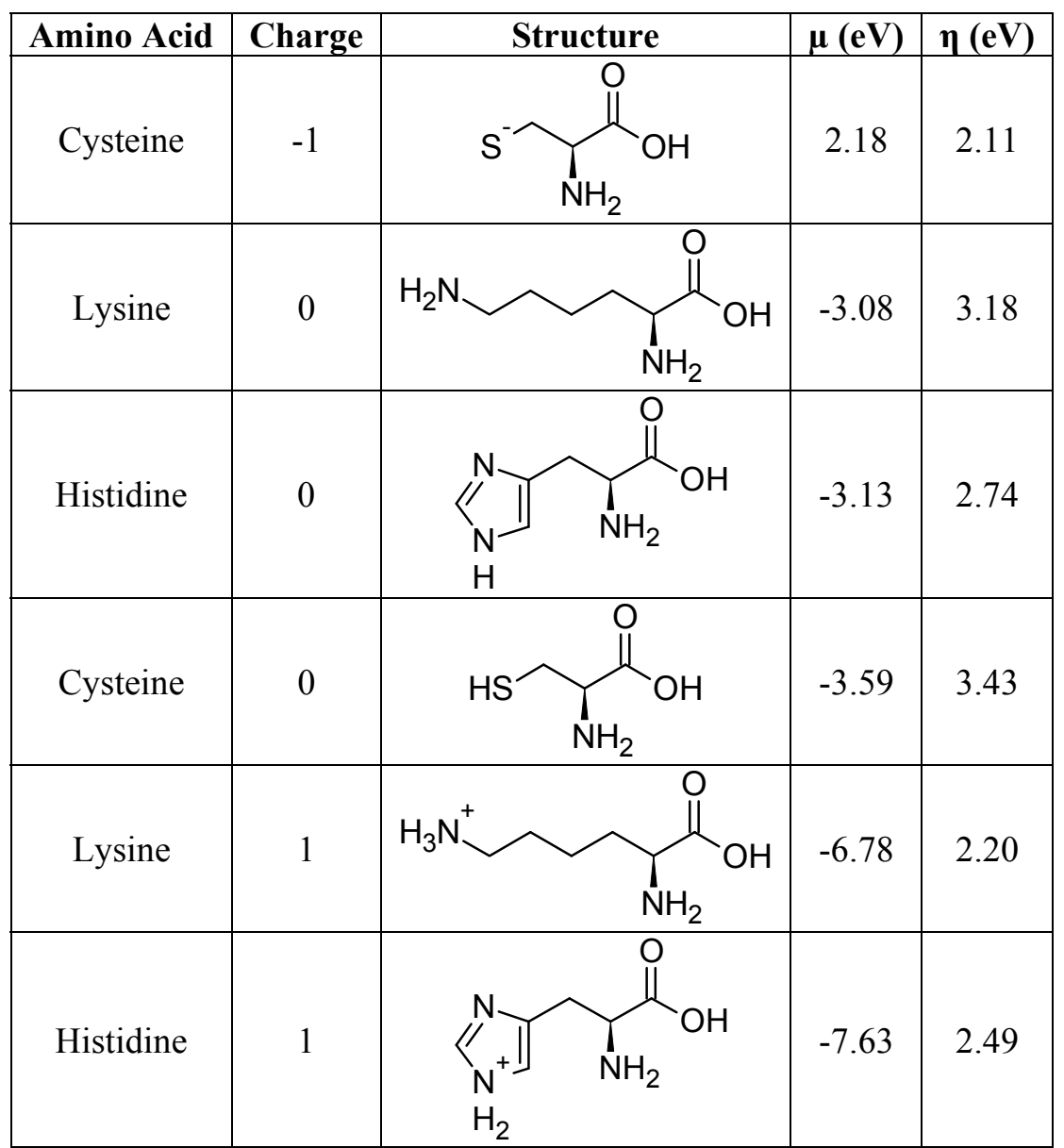

Table 10: Calculated chemical potentials and hardness values for amino acids studied in this work, as calculated by Equations (2) and (1) respectively.

Table 11 lists the calculated chemical potential and hardness values for other endogenous amino acids not studied in this work, but that may be considered potential alternative binding sites for electrophiles. As with Table 10, amino acids are ranked from highest chemical potential (better nucleophile) to lowest chemical potential. The values for Cys, Lys, and His are listed in red text so as to compare the amino acids to what was studied in this work.

As demonstrated by the results, there are several amino acids that have chemical potential values which suggest their utility as nucleophilic residues. However, these results should be approached with caution, as the $\mathrm{pK}_{\mathrm{a}}$ values of these residues are an important factor to consider.

For example, Asp and Glu have $\mathrm{pK}_{\mathrm{a}}$ values of 4.0 and 4.5 , respectively. ${ }^{57}$ Therefore, at 
physiological $\mathrm{pH}$ (7.4), the majority of these residues will be ionized (-1) and therefore good nucleophilic targets. However, Ser and Threonine (Thr) both have higher $\mathrm{pK}_{\mathrm{a}}$ values, which means that at physiological $\mathrm{pH}$, a low percentage of these residues will be ionized. As mentioned previously, there are steric and electronic factors within proteins that may produce residues at which the local $\mathrm{pK}_{\mathrm{a}}$ is lowered. Therefore, the above mentioned amino acids may be additional nucleophilic sites of interest for $\mathrm{HN}-2$ and $\mathrm{HN}-3$ adduction. It is also important to consider the amino acids which may not be charged, but may still have nucleophilic qualities possible for adduction. As noted in the table above, there are a few amino acids (Tryptophan, Tyrosine, and Arginine) that have chemical potential values high than neutral Lys and His, which have been demonstrated in this work to bind to HN-2 and HN-3. Consequently, these residues may also be potential nucleophilic targets for future study.

\begin{tabular}{|c|c|c|c|c|}
\hline Amino Acid & Charge & Structure & $\mu(\mathrm{eV})$ & $\eta(\mathrm{eV})$ \\
\hline Serine & -1 & & 2.49 & 2.19 \\
\hline Threonine & -1 & & 2.36 & 2.23 \\
\hline Cysteine & -1 & & 2.18 & 2.11 \\
\hline Aspartic Acid & -1 & & 1.45 & 2.22 \\
\hline Glutamic Acid & -1 & & 1.34 & 1.74 \\
\hline & & & & \\
\hline
\end{tabular}




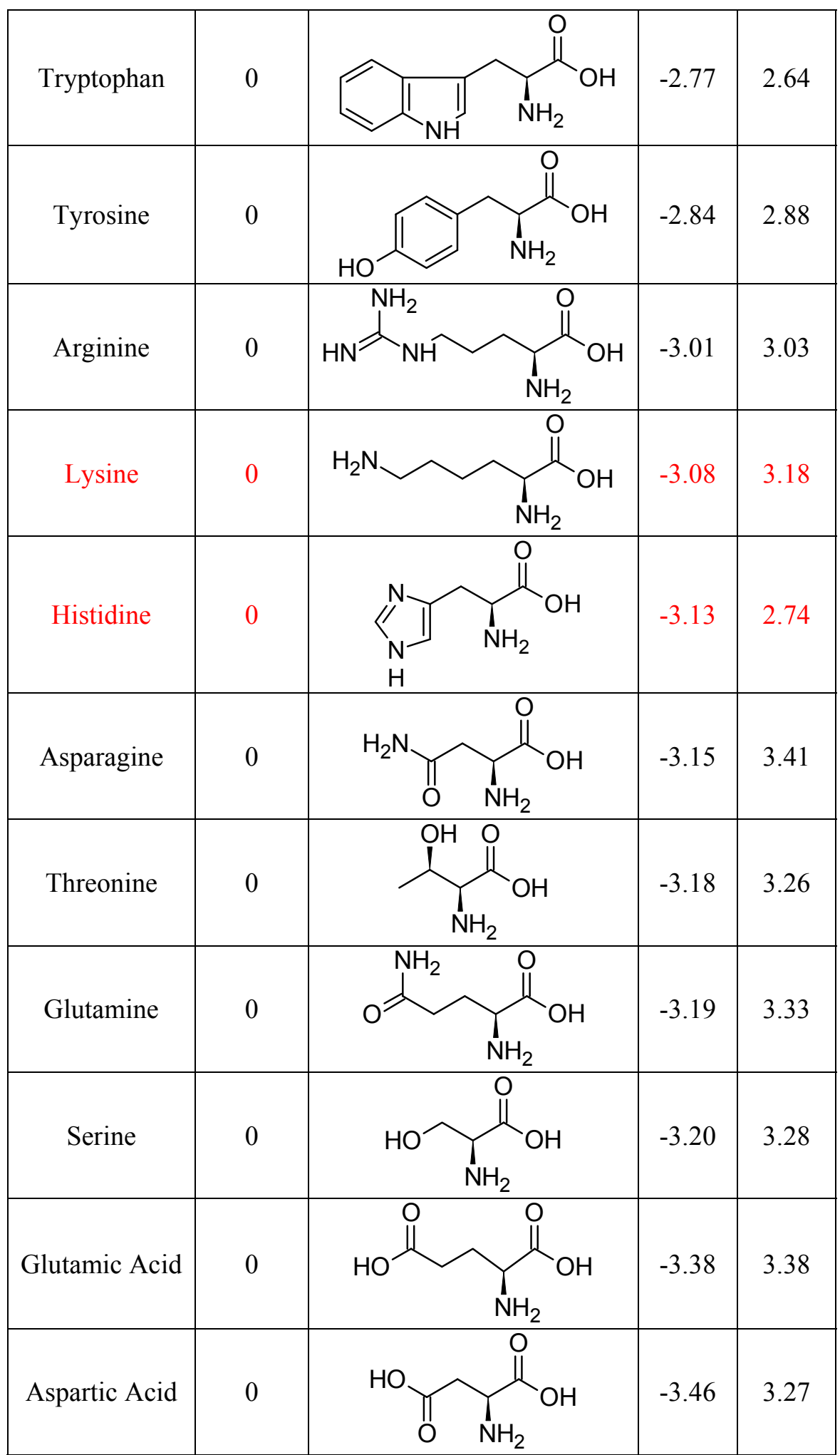




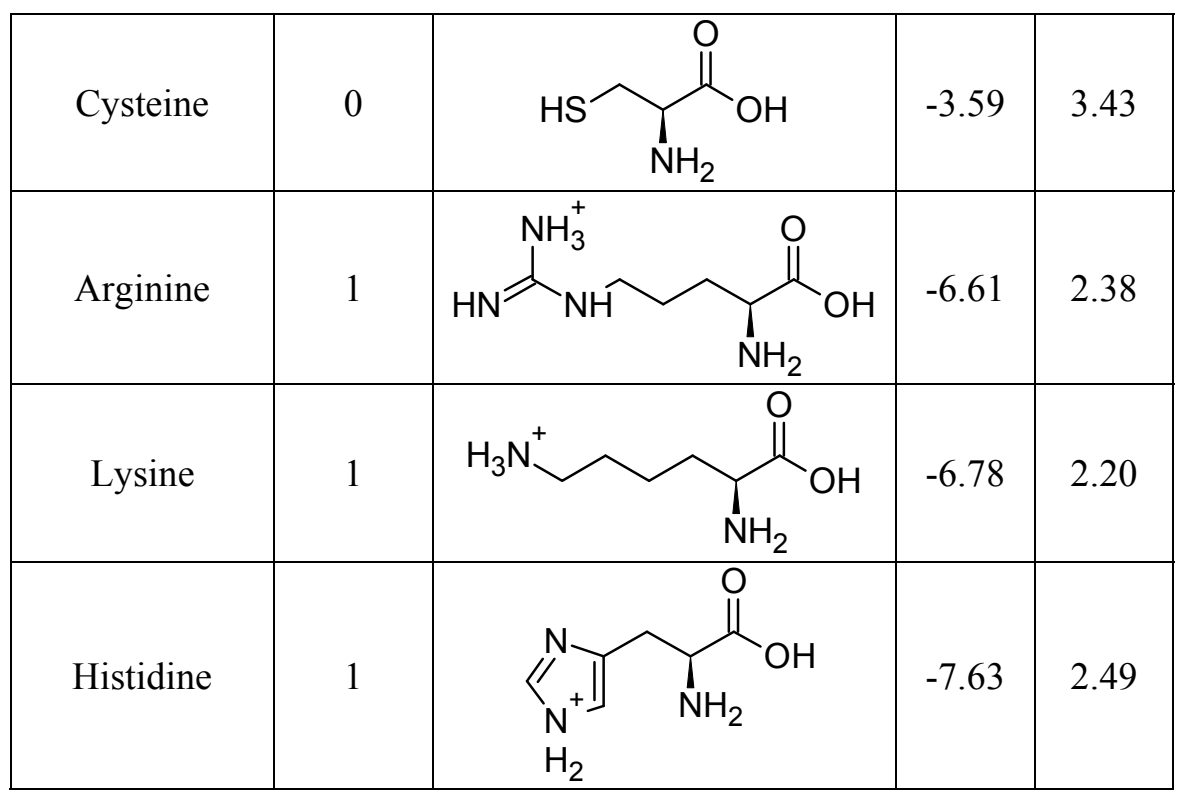

Table 11: Calculated chemical potential and hardness for ionizable endogenous amino acids, as calculated by Equations (2) and (1) respectively.

\subsubsection{Estimation of Nucleophile-Electrophile Interaction}

While chemical potential and hardness values provide some support for predictive aspects of electrophilic adduction to biological nucleophiles, a better indicator is the Reactivity Index ( $\omega-)$, as calculated via Equation 3. This value uses both calculated chemical potentials and hardness values to estimate how well a particular electrophile would react to a specific nucleophile. This parameter is important, as both chemical potential and hardness are factors in adduction. Table 12 demonstrates the reactivity index for the three amino acids studied in this work in conjunction with $\mathrm{HN}-2 \mathrm{Az} \mathrm{Cl}$ as the electrophile (reactivity index calculations for the $\mathrm{HN}-2 \mathrm{Az} \mathrm{OH}$ intermediate may be found in Appendix 9).

\begin{tabular}{|c|c|}
\hline \multicolumn{2}{|c|}{ Electrophile: HN-2 Az Cl } \\
\hline Amino Acid (Charge) & $\mathbf{\omega - ~ ( e V ) ~}$ \\
\hline Cysteine (-1) & 2.95 \\
\hline Lysine (0) & 0.75 \\
\hline Histidine (0) & 0.71 \\
\hline
\end{tabular}

Table 12: Calculated reactivity indices for $\mathrm{HN}-2 \mathrm{Az} \mathrm{Cl}$ to nucleophilic amino acids studied in this work, as calculated by Equation (3). 
As seen with previous results, $\mathrm{HN}-2 \mathrm{Az} \mathrm{Cl}$ is calculated to react more readily with Cys than with Lys and His - these results are supported by experimental data observed from the peptide relative adduction kinetics study. Since it has been demonstrated in this work that $\mathrm{HN}-2$ can also adduct to Lys and His, these calculated data can be used to provide a comparison for amino acids not directly studied in this work. Table 13 demonstrates the calculated reactivity index for other endogenous amino acids with $\mathrm{HN}-2 \mathrm{Az} \mathrm{Cl}$. The data for Cys, Lys, and His are highlighted in red for comparison purposes.

\begin{tabular}{|c|c|}
\hline \multicolumn{2}{|c|}{ Electrophile: HN-2 Az Cl } \\
\hline Amino Acid (Charge) & $\mathbf{\omega - ~ ( e V )}$ \\
\hline Serine (-1) & 3.17 \\
\hline Threonine (-1) & 3.11 \\
\hline Cysteine (-1) & 2.95 \\
\hline Aspartic Acid (-1) & 2.58 \\
\hline Glutamic Acid (-1) & 2.32 \\
\hline Tryptophan (0) & 0.82 \\
\hline Tyrosine (0) & 0.81 \\
\hline Arginine (0) & 0.76 \\
\hline Lysine (0) & 0.75 \\
\hline Asparagine (0) & 0.73 \\
\hline Threonine (0) & 0.72 \\
\hline Glutamine (0) & 0.72 \\
\hline Histidine (0) & 0.71 \\
\hline Serine (0) & 0.71 \\
\hline Glutamic Acid (0) & 0.66 \\
\hline Aspartic Acid (0) & 0.64 \\
\hline Cysteine (0) & 0.60 \\
\hline Arginine (+1) & 0.05 \\
\hline Lysine (+1) & 0.04 \\
\hline Histidine (+1) & 0.003 \\
\hline
\end{tabular}

Table 13: Calculated reactivity index for $\mathrm{HN}-2 \mathrm{Az} \mathrm{Cl}$ for ionizable amino acids as calculated by Equation (3).

The table suggests that, given the right conditions, HN-2 could bind to other amino acids besides Cys, Lys, and His. As mentioned earlier, Ser and Thr appear to be appropriate choices for future studies, as their neutral forms have reactivity indexes similar to that of Lys and His. Additionally, reactivity index data for Tyr and Tryptophan (Trp) indicate potential vulnerability 
as additional binding sites for $\mathrm{HN}$, as these are known targets for electrophilic adduction by other xenobiotics. $^{55}$

Table 14 demonstrates the reactivity index data for $\mathrm{HN}-3 \mathrm{Az} \mathrm{Cl} \mathrm{Cl}$ for the amino acids studied in this work (calculated data for the other HN-3 intermediates may be found in Appendix 9). Nucleophiles are ranked from highest to lowest calculated reactivity index value.

\begin{tabular}{|c|c|}
\hline \multicolumn{2}{|c|}{ Electrophile: HN-3 Az Cl Cl } \\
\hline Amino Acid (Charge) & $\mathbf{\omega - ~ ( e V )}$ \\
\hline Cysteine (-1) & 3.19 \\
\hline Lysine (0) & 0.82 \\
\hline Histidine (0) & 0.79 \\
\hline
\end{tabular}

Table 14: Calculated reactivity index values for $\mathrm{HN}-3 \mathrm{Az} \mathrm{Cl} \mathrm{Cl}$ to nucleophilic amino acids studied in this work, as calculated by Equation (3).

As demonstrated previously, $\mathrm{HN}-3 \mathrm{Az} \mathrm{Cl} \mathrm{Cl}$ is expected to more readily react with Cys thiolate than with Lys or His. These values can be used as comparison for other amino acids not tested in this work, as can be seen in Table 15. The values for Cys, Lys, and His are highlighted in red. 


\begin{tabular}{|c|c|}
\hline \multicolumn{2}{|c|}{ Electrophile: HN-3 Az Cl Cl } \\
\hline Amino Acid (Charge) & $\mathbf{\omega - ~ ( e V )}$ \\
\hline Serine (-1) & 3.43 \\
\hline Threonine (-1) & 3.36 \\
\hline Cysteine (-1) & 3.19 \\
\hline Aspartic Acid (-1) & 2.80 \\
\hline Glutamic Acid (-1) & 2.53 \\
\hline Tryptophan (0) & 0.90 \\
\hline Tyrosine (0) & 0.89 \\
\hline Arginine (0) & 0.84 \\
\hline Lysine (0) & 0.82 \\
\hline Asparagine (0) & 0.80 \\
\hline Threonine (0) & 0.79 \\
\hline Histidine (0) & 0.79 \\
\hline Glutamine (0) & 0.79 \\
\hline Serine (0) & 0.79 \\
\hline Glutamic Acid (0) & 0.73 \\
\hline Aspartic Acid (0) & 0.70 \\
\hline Cysteine (0) & 0.67 \\
\hline Arginine (+1) & 0.07 \\
\hline Lysine (+1) & 0.05 \\
\hline Histidine (+1) & 0.007 \\
\hline
\end{tabular}

Table 15: Calculated Reactivity Index values for $\mathrm{HN}-3 \mathrm{Az} \mathrm{Cl} \mathrm{Cl}$ and ionizable amino acids, as calculated by Equation (3).

As with $\mathrm{HN}-2$, the results from $\mathrm{HN}-3$ suggest that there may be other amino acids that could be adduction targets for $\mathrm{HN}-3$ and that merit future study.

\subsubsection{Discussion}

The calculation of reactivity indices discussed above serves a dual purpose. First, the chemical potential data obtained for $\mathrm{HN}-2, \mathrm{HN}-3$, and respective electrophilic intermediates lends additional support for the hypothesis regarding Az binding of proteins and DNA. In addition, the calculated hardness values obtained for these electrophilic species, as compared to a known soft electrophile, demonstrate that these compound exhibit characteristics of harder electrophiles, meaning that they have the potential for adducting a variety of nucleophilic centers. In addition, the calculated hardness values for uncharged Lys and His are comparable, suggesting that they would react similarly with electrophiles. Again, this is supported by the model peptide work in which the extent of adduction by $\mathrm{HN}-2$ and $\mathrm{HN}-3$ to these sites was similar. 
Based on the calculated data, the Cys thiolate appears to be the nucleophile most likely to react with electrophilic $\mathrm{HN}-2$ and $\mathrm{HN}-3 \mathrm{Az}$ species. This is supported by the model peptide experiments, in which the rate of formation of Cys adducts was greater than for Lys and His.

Second, the calculated reactivity data suggests additional amino acids that may be potential adduction sites for $\mathrm{HN}-2$ and $\mathrm{HN}-3$ besides the three studied in this work. However, along with these predictions, it is important to consider how these amino acids are presented under biological conditions, specifically in terms of $\mathrm{pK}_{\mathrm{a}}$ and ionization. The $\mathrm{pK}_{\mathrm{a}}$ of a particular amino acid will determine whether the side chain is charged or neutral, and at what general percentage it would be expected to be in this charged or neutral state. This factor is important when considering, for example, the results calculated for Lys and His. The $\mathrm{pK}_{\mathrm{a}}$ of Lys is 10.4, whereas the $\mathrm{pK}_{\mathrm{a}}$ of His is $6.4 .^{57}$ This means that, at $\mathrm{pH} 7.4$, virtually no $(0.1 \%)$ Lys residues will be unionized, whereas $90 \%$ of His residues are unionized. Therefore, despite the fact that calculated reactivity values for these two residues are relatively comparable, it would be expected that, all other factors being equal, a greater fraction of His residues would be adducted as compared to Lys. This lends support to the obtained experimental data, in which the majority of adducted residues determined on full proteins were His. However, as discussed previously, electronic and steric factors surrounding individual amino acids may provide a local $\mathrm{pK}_{\mathrm{a}}$ for a specific residue lower than expected, which could render a more favorable adduction site. This may be the case for the specific Lys residues adducted on HSA identified in this study. Further work would be required to determine the local $\mathrm{pK}_{\mathrm{a}}$ of these Lys residues based on the amino acids surrounding the adduction site. 


\section{SUMMARY AND PROSPECT}

The goal of this project was to identify novel and specific potential exposure biomarkers for the chemical warfare agents $\mathrm{HN}-2$ and $\mathrm{HN}-3$ based on adduct formation on the blood proteins $\mathrm{Hb}$ and HSA. This work has identified and characterized four previously unknown adduction sites on $\mathrm{Hb}$ and an additional four sites on HSA, all involving Lys and His modification. Adducts formed by $\mathrm{HN}-2$ and $\mathrm{HN}-3$ at these specific sites were determined to be stable during a threeweek analysis period and were confirmed in a proof-of-concept study involving in vitro exposure of these agents to human whole blood. Characterized adducts were specific to HN-2 or HN-3, and were not observed with three common nitrogen mustard therapeutic agents following in vitro metabolism. This work also demonstrated, using theoretical quantum mechanical calculations, that other additional amino acid residues may provide additional sites of adduction for the nitrogen mustard CWA. This work represents a crucial step in the development of useful, longerterm exposure biomarkers for this class of forensically relevant compounds.

The identification of novel exposure biomarkers for $\mathrm{HN}-2$ and $\mathrm{HN}-3$ has great potential for forensic analysis. The adducts identified here have the ability to extend the window of detection of these compounds as compared to shorter lived ethanolamine metabolites, as demonstrated by the stability assessment of these adducts both on model peptides and full proteins. In addition, these protein adducts allow for a level of specificity for $\mathrm{HN}$ exposure that is not available with the ethanolamine metabolites. Once validated, these biomarkers could be utilized for testing individuals poisoned with nitrogen mustard CWA, or, perhaps more importantly, to identify subjects seeking to manufacture and disperse such materials in terrorist attacks. Finally, these markers could provide definitive evidence of HN CWA exposure distinct from other nitrogen mustard containing compounds, including therapeutic compounds.

There are additional avenues that may be considered for future development of adductbased exposure markers for $\mathrm{HN}$ and other related compounds. First, as mentioned above, 
quantum mechanical calculations suggest that there may be additional amino acid binding sites for these compounds. Future studies with other reactive sites, such as Asp and Glu, may not only yield additional potential exposure biomarkers, but may also provide clues regarding the toxic mechanism of these compounds. For example, as these two amino acids are found in abundance in keratin, ${ }^{87}$ adduct studies with this protein may provide additional insight into the molecular basis of blister agent effects on skin.

Second, the current findings may also be directly applicable to the more notorious sulfur mustard in terms of potential exposure biomarkers. While HD adduction to His has been documented $^{83}$ to a limited degree, improvements in LC-MS and LC-MS-MS technology since these earlier studies may allow for identification of specific adducted residues for this related CWA. Finally, while the present work focused on the initial identification of these adducts, the conditions utilized for their reliable identification (in particular, the relatively high levels of $\mathrm{HN}$ required for adduct detection and reproducibility) may be best suited to modeling acute, highlevel as opposed to chronic, low-level exposure. Additional work should be done in which more sensitive methods are utilized to lower the detection limit for adduct analysis. For example, specific adducted tryptic peptides could be synthesized and utilized for MS and MS-MS parameter optimization (such as fragmentor voltage and collision energy). In addition, such standards could be used for MRM optimization and detection via QQQ, which would provide a level of sensitivity that cannot be achieved with current Q-TOF instrumentation. As detection technology becomes more sensitive, these novel protein adducts will have increasing potential as exposure biomarkers for these agents at lower exposure levels, potentially to the point at which no overt symptoms are present in exposed individuals. These protein adducts could then be employed as sensitive, specific, and stable biomarkers of exposure to the nitrogen mustard chemical warfare agents $\mathrm{HN}-2$ and $\mathrm{HN}-3$. 


\section{REFERENCES}

1. Convention on the Prohibition of the Development, Production, Stockpiling, and Use of Chemical Weapons and on their Destruction; Organization for the Prohibition of Chemical Weapons: Hauge, the Netherlands, 1993.

2. Szinicz, L. History of Chemical and Biological Warfare Agents. Toxicology 2005, 214, 167-181.

3. Collina, T. Z. Chemical Weapsons Convention Signatories and State-Parties. http://www.armscontrol.org/factsheets/cwcsig (Accessed December 10, 2013)

4. Seto, Y. The Sarin Gas Attack in Japan and the Related Forensic Investigation. $O P C W$ Synthesis 2001, 14-17.

5. Sellström, Å.; Cairns, S.; Barbeschi, M. United Nations Mission to Investigate Allegations of the Use of Chemical Weapons in the Syrian Arab Republic; Report Number A/67/997-S/2013/553; Organization for the Prohibition of Chemical Weapons: Hauge, the Netherlands, September 13, 2013.

6. Sommargren, M.; Karr, C. Organophosphate Pesticides and Child Health: A Primer for Health Care Providers. http://depts/washington.edu/opchild/index.html (Accessed December 17, 2013). Last updated 2007.

7. Chauhan, S.; Chauhan, S.; D'Cruz, R.; Faruqi, S.; Singh, K. K.; Varma, S.; Singh, M.; Karthik, V. Chemical Warfare Agents. Environmental Toxicology and Pharmacology 2008, 26, 113-122.

8. Elhanany, E.; Ordentlich, A.; Dgany, O.; Kaplan, D.; Segall, Y.; Barak, R.; Velan, B.; Shafferman, A. Resolving Pathways of Interaction of Covalent Inhibitors with the Active Site of Acetylcholinesterases: MALDI-TOF/MS Analysis of Varios Nerve Agents Phosphyl Adducts. Chemical Research in Toxicology 2001, 14, 912-918.

9. Cholinesterase Inhibitors: Including Insectisides and Chemical Warfare Nerve Agents. Agency for Toxic Substances and Disease Registry Part 4 Section 11:

Management Strategies 3: Medications.

http://www.atsdr.cdc.gov/csem/csem.asp?csem $=11 \& p o=23$ (Accessed December 10, 2013) Last updated October 16, 2010.

10. John, H.; Worek, F.; Thiermann, H. LC-MS-based procedures for monitoring of toxic organophosphorus compounds and verification of pesticide and nerve agent poisoning. Analytical and Bioanalytical Chemistry 2008, 391 (1), 97-116.

11. Nassar, A. E. F.; Lucas, S. V.; Myler, C. A. Quantitative Analysis of Chemical Warfare Agent Degradation Products in Reaction Masses Using Capillary Electrophoresis. Analytical Chemistry 1998, 70, 3598-3604.

12. Kataoka, M.; Seto, Y. Discriminative determination of alkyl methylphosphonates and methylphosphonate in blood plasma and urine by gas chromatography-mass 
spectrometry ater tert-butyldimethylsilylation. Journal of Chromatography $B$ 2003, 795, 123-132.

13. Terzic, O. Screening of degradation products, impurities and precursors of chemical warfare agents in water and wet or dry organic liquid samples by in-sorbent tube silylation followed by thermal desorption-gas chromatography-mass spectrometry. Journal of Chromatography A 2010, 1217, 4987-4995.

14. Kientz, C. E. Chromatography and mass spectrometry of chemical warfare agents, toxins and related compounds: state of the art and future prospects. Journal of Chromatography A 1998, 814 (1-2), 1-23.

15. Evans, R. A.; Jakubowski, E. M.; Muse, W. T.; Matson, K.; Hulet, S. W.; Mioduszewski, R. J.; Thomson, S. A.; Totura, A. L.; Renner, J. A.; Crouse, C. L. Quantification of Sarin and Cyclosarin Metabolites Isopropyl Methlyphosphonic Acid and CyclohexylMethylphosphonic Acid in Minipig Plasma Using Isotope-Dilution and Liquid Chromatography-Time-of-Flight Mass Spectrometry. Journal of Analytical Toxicology 2008, (32), 78-85.

16. Holland, K. E.; Solano, M. I.; Johnson, R. C.; Maggio, V. L.; Barr, J. R. Modification to the organophosphorus nerve agent-protein adduct refluoridation method for retrospective analysis of nerve agent exposures. Journal of Analytical Toxicology 2008, 32 (1), 116-124.

17. Renner, J. A.; Dabisch, P. A.; Evans, R. A.; McGuire, J. M.; Totura, A. L.; Jakubowski, E. M.; Thomson, S. A. Validation and Application of a GC-MS Method for Determining Soman Concentration in Rat Plasma Following Low-Level Vapor Exposure. Journal of Analytical Toxicology 2008, 32, 92-98.

18. Degenhardt-Langelaan, C. E. A. M.; Pleijser, K.; van der Schans, M. J.; Langenberg, J. P.; Preston, K. E.; Solano, M. I.; Maggio, V. L.; Barr, J. R. Improvements of the Fluoride Reactivation Method for the Verification of Nerve Agent Exposure. Journal of Analytical Toxicology 2004, 28, 364-371.

19. Adams, T. K.; Capacio, B. R.; Smith, J. R.; Whalley, C. E.; Korte, W. D. The Application of the Fluoride Reactivation Process to the Detection of Sarin and Soman Nerve Agent Exposures in Biological Samples. Drug and Chemical Toxicology 2004, $27(1), 77-91$.

20. Tsuge, K.; Seto, Y. Detection of human butyrylcholinesterase-nerve gas adducts by liquid chromatography-mass spectrometric analysis after in gel chymotryptic digestion. Journal of Chromatography B 2006, 838, 21-30.

21. van der Schans, M. J.; Fidder, A.; van Oeveren, D.; Hulst, A. G.; Noort, D. Verification of Exposure to Cholinesterase Inhibitors: Generic Detection of OPCW Schedule 1 Nerve Agent Adducts to Human Butyrylcholinesterase. Journal of Analytical Toxicology 2008, 32, 125-130.

22. Young, R. A.; Bast, C. Mustards and Vesicants. In Handbook of Toxicology of Chemical Warfare Agents, 1st ed.; 2009; pp 93-108. 
23. Sharma, M.; Vijayaraghavan, R.; Agrawal, O. P. Comparative Toxic Effect of Nitrogen Mustards (HN-1, HN-2, and HN-3) and Sulfur Mustard on Hematological and Biochemical Variables and Their Protection by DRDE-07 and its Analogues. International Journal of Toxicology 2010, 29 (4), 391-401.

24. Balcome, S.; Park, S.; Dorr, D. R. Q.; Hafner, L.; Phillips, L.; Tretyakova, N. Adeninecontaining DNA-DNA cross-links of antitumor nitrogen mustards. Chemical Research in Toxicology 2004, 17 (7), 950-962.

25. Hemminki, K.; Kallama, S. Reactions of Nitrogen Mustard with DNA. IARC Scientific Publications 1986, 78, 55-70.

26. Chain, P. K. Cross-Linkage of Nucleophosmin in Tumor Cells by Nitrogen Mustard. Cancer Research 2013, 49 (3271), 3275.

27. Sayer, N. M.; Whiting, R.; Green, A. C.; Anderson, K.; Jenner, J.; Lindsay, C. D. Direct binding of sulfur mustard and chloroethyl ethyl sulphide to human cell membrane-associated proteins; implications for sulfur mustard pathology. Journal of Chromatography B 2009, 878, 1426-1432.

28. Fidder, A.; Noort, D.; Hulst, A. G.; de Jong, L. P. A.; Benschop, H. P. Biomonitoring of exposure to lewisite based on adducts to haemoglobin. Archives of Toxicology 2000, 74 (4-5), 207-214.

29. Mann, D. J. Aziridinium Ion Ring Formation from Nitrogen Mustards: Mechanistic Insights from Ab Initio Dynamics. Journal of Physical Chemistry A 2010, 114 (13), 4486-4493.

30. Stewart, C. E. Vesicant Agents. In Weapns of Mass Casualties and Terrorism Response Handbook, Morris, J., McIssac, J., Eds.; Jones and Bartlett Publishers: Sudburry, MD, 2006; pp 46-59.

31. Ucar, M.; Korkmaz, A.; Reiter, R. J.; Yaren, H.; Oter, S.; Kurt, B.; Topal, T. Melatonin alleviates lung damage induced by the chemical warfare agent nitrogen mustard. Toxicology Letters 2007, 173, 124-131.

32. Malaviya, R.; Sunil, V. R.; Cervelli, J.; Anderson, D. R.; Homles, W. W.; Conti, M. L.; Gordon, R. E.; Laskin, J. D.; Laskin, D. L. Inflammatory effects of inhaled sulfur mustard in rat lung. Toxicology and Applied Pharmacology 2010, 248, 88-99.

33. Korkmaz, A.; Yaren, H.; Topal, T.; Oter, S. Molecular targets against mustard toxicity: implication of cell surface receptors, peroxynitrite production, and PARP activation. Archives of Toxicology 2013, 80, 662-670.

34. Cowan, F. M.; Broomfield, C. A.; Lenz, D. E.; Smith, W. J. Putative Role of Proteolysis and Inflammatory Response in the Toxicity of Nerve and Blister Chemical Warfare Agents: Implications for Multi-threat Medical Countermeasures. Journal of Applied Toxicology 2003, 23, 177-186. 
35. Shakarjian, M. P.; Heck, D. E.; Gray, J. P.; Sinko, P. J.; Gordon, M. K.; Casillas, R. P.; Heindel, N. D.; Gerecke, D. R.; Laskin, D. L.; Laskin, J. D. Mechanisms Mediating the Vesicant Acition of Sulfur Mustard after Cutaneous Exposure. Toxicological Sciences 2009, 114 (1), 5-19.

36. Chua, H. C.; Lee, H. S.; Sng, M. T. Screening of nitrogen mustards and their degradation products in water and decontamination solution by liquid chromatography-mass spectrometry. Journal of Chromatography A 2006, 1102 (1-2), 214-223.

37. Wang, Q.-Q.; Begum, R. A.; Day, V. W.; Bowman-James, K. Sulfur, Oxygen, and Nitrogen Mustards: Stability and Reactivity. Organic and Biomolecular Chemistry 2012, 10, 8786-8793.

38. Kinoshita, K.; Shikino, O.; Seto, Y.; Kaise, T. Determination of degradation compounds derived from Lewisite by high performance liquid chromatography/inductively coupled plasma-mass spectrometry. Applied Organometallic Chemistry 2006, 20, 591-596.

39. Black, R. M.; Noort, D. Methods for Retrospective Detection of Exposure to Toxic Scheduled Chemicals. Part A: Analysis of Free Metabolites. In Chemical Weapons Convention Chemical Analysis, Markku Mesilaakso, Ed.; John Wiley \& Sons: 2005; pp 403-429.

40. Dubey, D. K.; Pardasani, D.; Palit, M.; Gupta, A. K.; Jain, R. On-matrix derivatisationextraction of precursors of nitrogen- and sulfur-mustards for verification of chemical weapons convention. Journal of Chromatography A 2013, 1076, 27-33.

41. Gamcsik, M. P.; Hamil, T. G.; Colvin, M. E. NMR Studies of the Conjugation of Mechloroethamine with Glutathione. Journal of Medicinal Chemistry 1990, 33, 1009-1014.

42. Hambrook, J. L.; Howells, D. J.; Schock, C. Biological fate of sulphur mustard (1,1'thiobis(2-chloroethane)): uptake, distribution, and retention of ${ }^{35} \mathrm{~S}$ in skin and in blood after cutaneous application of ${ }^{35} \mathrm{~S}$-sulphur mustard in rat and comparison with human blood in vitro. Xenobiotica 1993, 23 (5), 537-561.

43. Snider, T. H.; Wientjes, M. G.; Joiner, R. L.; Fisher, G. L. Arsenic Distrubution in Rabbits after Lewisite Administration and Treatment with Biritsh Anti-Lewisite (BAL). Fundamental and Applied Toxicology 1990, 14, 262-272.

44. Pastore, A.; Federici, G.; Bertini, E.; Piemonte, F. Analysis of glutathione: implication in redox and detoxification. Clinica Chimica Acta 2003, 333, 19-39.

45. Stocken, L. A.; Thompson, R. H. S. British Anti-Lewisite 1. Arsenic Derivatives of Thiol Proteins. Biochemical Journal 1946, 40 (4), 529-535.

46. Alaoui-Jamali, M. A.; Panasci, L.; Centurioni, G. M.; Schecter, R.; Lehnert, S.; Batist, G. Nitrogen mustard-DNA interaction in melphalan-resistant mammary carcinoma cells with elevated intracellular glutathion and glutathione-S-transferase activity. Cancer Chemotherapy and Pharmacology 1992, 30, 341-347. 
47. Ning, Z.; Zhou, H.; Wang, F.; Abu-Farha, M.; Figeys, D. Analytical Aspects of Proteomics: 2009 - 2010. Analytical Chemistry 2011, 83, 4407-4426.

48. Smith, W. J.; Salem, H. Biomarkers of Chemical Warfare Agents. 2005; pp 259-272.

49. DeCaprio, A. P. Biomarkers of Exposure and Susceptibility. In General and Applied Toxicology, 2nd ed.; Ballantyne, B., Marrs, T. C., Syversen, T., Eds.; MacMillan Reference Ltd.: London, 2000; pp 1875-1898.

50. Noort, D.; Benschop, H. P.; Black, R. M. Biomonitoring of exposure to chemical warfare agents: A review. Toxicology and Applied Pharmacology 2002, 184 (2), 116-126.

51. Pearson, R. G. Chemical hardness and density functional theory. J. Chem. Sci 2005, 117 (5), 369-377.

52. Black, R. M. An overview of biological markers of exposure to chemical warfare agents. Journal of Analytical Toxicology 2008, 32 (1), 2-9.

53. Kenar, L.; Alp, O. Determination of Nitrogen Mustard Hydrolysis Products in Rat Urine Samples using GC-MS. Journal of Chromatographic Science 2011, 49, 361-364.

54. Törnqvist, M.; Fred, C.; Haglund, J.; Helleberg, H.; Paulsson, B.; Rydberg, R. Protein adducts: quantitative and qualitative aspects of their formation, analysis and applications. Journal of Chromatography B-Analytical Technologies in the Biomedical and Life Sciences 2002, 778 (1-2), 279-308.

55. Schnell, F. C. Protein Adduct-Forming Chemicals and Molecular Dosimetry: Potential for Environmental and Occupational Biomarkers. Reviews in Environmental Toxicology 1994, 5, 51-160.

56. Zhou, S.; Chan, E.; Duan, W. D.; Huang, M.; Chen, Y. Z. Drug Bioactivation, Covalent Binding to Target Proteins and Toxicity Relevance. Drug metabolism Reviews 2005, $1,41-213$.

57. Harris, T. K.; Turner, G. J. Structural Basis of Perturbed pKa Values of Catalytic Groups in Enzyme Active Sites. Life 2002, 53, 85-98.

58. LoPachin, R. M.; Barber, D. S. Synaptic Cysteine Sulfhydryl Groups as Targets of Electrophilic Neurotoxins. Toxicological Sciences 2006, 94 (2), 240-255.

59. Isom, D. G.; Castañeda, C. A.; Cannon, B. R.; García-Moreno, B. Large shifts in pKa values of lysine residues buried inside a protein. Proc. Natl. Acad. Sci. USA 2010, 108 (13), 5260-5265.

60. Edgecomb, S. P.; Murphy, K. P. Variability in the pKa of Histidine Side-Chains Correlates With Burial Within Proteins. Proteins: Structure, Function, and Genetics 2002, 49, 1-6.

61. LoPachin, R. M.; DeCaprio, A. P. Protein adduct formation as a molecular mechanism in neurotoxicity. Toxicological Sciences 2005, 86 (2), 214-225. 
62. LoPachin, R. M.; Gavin, T.; DeCaprio, A. P.; Barber, D. S. Application of the Hard and Soft, Acids and Bases (HSAB) Theory to Toxicant-Target Interactions. Chemical Research in Toxicology 2012, 25, 239-251.

63. Jaramillo, P.; Perez, P.; Contrearas, R.; Tiznado, W.; Fuentealba, P. Definition of a Nucleophilicity Scale. Journal of Physical Chemistry 2006, 110, 8181-8187.

64. Rauk, A. Molecular Orbital Theory. In Orbital Interaction Theory of Organic Chemistry, 2nd Edition ed.; John Wiley \& Sons: New York, 2001; pp 20-33.

65. Carpenè, E.; Andreani, G.; Isani, G. Metallothionein functions and structural characteristics. Journal of Trace Elements in Medicine and Biology 2007, 21, 3539.

66. Anderson, R. D.; Winter, W. P.; Maher, J. J.; Bernstein, I. A. Turnover of Metallothioneins in Rat Liver. Biochemical Journal 1978, 174, 327-338.

67. Hsia, C. C. W. Respiratory Function of Hemoglobin. The New England Journal of Medicine 1998, 338 (4), 239-247.

68. Poppek, D.; Grune, T. Protein Repair and Degradation. In Oxidants and Antioxidant Defense Systems, Tilman, G., Ed.; Springer: Berlin, 205; pp 177-201.

69. Barak, D.; Ordentlich, A.; Kaplan, D.; Barak, R.; Mizrahi, D.; Kronman, C.; Segall, Y.; Velan, B.; Shafferman, A. Evidence for P-N Bond Scission in Phosphoramide Nerve Agent Adducts of Human Acetylcholinesterase. Biochemistry 2000, 39, 1156-1161.

70. Lui, G.; Wang, J.; Barry, R.; Petersen, C.; Timchalk, C.; Gassman, P. L.; Lin, Y. Nanoparticle-Based Electrochemical Immunosensor for the Detection of Phosphorylated Acetycholinesterase: An Exposure Biomarker of Organophosphate Pesticides and Nerve Agents. Chemistry: A European Journal 2008, 14 (32), 9951-9959.

71. Carletti, E.; Li, H.; Li, B.; Ekstrom, F.; Nicolet, Y.; Loiodice, M.; Gillon, E.; Froment, M. T.; Lockridge, O.; Schopfer, L. M.; Masson, P.; Nachon, F. Aging of Cholinesterases Phosphorylated by Tabun Proceeds through O-Dealkylation. Journal of the American Chemical Society 2008, 130, 16011-16020.

72. Williams, N. H.; Harrison, J. M.; Read, R. W.; Black, R. M. Phosphylated tyrosine in albumin as a biomarker of exposure to organophosphorus nerve agents. Archives of Toxicology 2007, 81 (9), 627-639.

73. Li, B.; Nachon, F.; Froment, M. T.; Verdier, L.; Debouzy, J. C.; Brasme, B.; Gillon, E.; Schopfer, L. M.; Lockridge, O.; Masson, P. Binding and hydrolysis of soman by human serum albumin. Chemical Research in Toxicology 2008, 21 (2), 421-431.

74. Black, R. M.; Harrison, J. M.; Read, R. W. The interaction of sarin and soman with plasma proteins: the identification of a novel phosphonylation site. Archives of Toxicology 1999, 73 (2), 123-126. 
75. Schopfer, L. M.; Lockridge, O. Analytical approaches for monitoring exposure to organophosphorous and carbamate agents through analysis of protein adducts. Drug Testing and Analysis 2012, 4, 246-261.

76. Li, B.; Eyer, P.; Eddleston, M.; Jiang, W.; Schopfer, L. M.; Lockridge, O. Protein tyrosine adduct in humans self-poisoned by chlorpyrifos. Toxicology and Applied Pharmacology 2013, 269, 215-225.

77. Byers, C. E.; McGuire, J. M.; Hulet, S. W.; Burnett, D. C. G. B. I.; Jakubowski, E. M.; Thomson, S. A. Gas Chromatography-Tandem Mass Spectrometry Analysis of Red Blood Cells from Gottingen Minipig following Whole-Blood Vapor Exposure to VX. Journal of Analytical Toxicology 2008, 32, 57-62.

78. McGuire, J. M.; Byers, C. E.; Hulet, S. W.; Jakubowski, E. M.; Thomson, S. A. A Rapid and Sensitive Technique for Assessing Exposure to VX via GC-MS-MS Analysis. Journal of Analytical Toxicology 2008, 32, 63-67.

79. Carol-Visser, J.; van der Schans, M.; Fidder, A.; Huist, A. G.; van Baar, B. L. M.; Irth, H.; Noort, D. Development of an automated on-line pepsin digestion-liquid chromatography-tandem mass spectrometry configuration for the rapid analysis of protein adducts of chemical warfare agents. Journal of Chromatography BAnalytical Technologies in the Biomedical and Life Sciences 2008, 870 (1), 9197.

80. Noort, D.; Hulst, A. G.; de Jong, L. P. A.; Benschop, H. P. Alkylation of human serum albumin by sulfur mustard in vitro and in vivo: Mass spectrometric analysis of a cysteine adduct as a sensitive biomarker of exposure. Chemical Research in Toxicology 1999, 12 (8), 715-721.

81. Capacio, B. R.; Smith, J. R.; Lawrence, R. J.; Boyd, B. L.; Witriol, A. M.; Conti, M. L.; Collins, J. L.; Sciuto, A. M. Gas Chromatographic-Mass Spectrometric Analysis of Sulfur Mustard-Plasma Protein Adducts: Validation and Use in a Rat Inhalation Model. Journal of Analytical Toxicology 2008, 32, 37-43.

82. Noort, D.; Fidder, A.; Degenhardt-Langelaan, C. E. A. M.; Hulst, A. G. Retrospective detection of sulfur mustard exposure by mass spectrometric analysis of adducts to albumin and hemoglobin: An in vivo study. Journal of Analytical Toxicology 2008, 32 (1), 25-30.

83. Noort, D.; Hulst, A. G.; Trap, H. C.; Dejong, L. P. A.; Benschop, H. P. Synthesis and mass spectrometric identification of the major amino acid adducts formed between sulphur mustard and haemoglobin in human blood. Archives of Toxicology 1997, 71 (3), 171-178.

84. Black, R. M.; Clarke, R. J.; Harrison, J. M.; Read, R. W. Biological fate of sulphur mustard: Identification of valine and histidine adducts in haemoglobin from casualties of sulphur mustard poisoning. Xenobiotica 1997, 27 (5), 499-512. 
85. Nie, Z.; Lui, Q.; Xie, J. Improvements in monitoring the N-terminal valine adduct in human globin after exposure to the sulfur mustard and synthesis of reference chemicals. Talanta 2011, (85), -1154.

86. Noort, D.; Fidder, A.; Hulst, A. G.; de Jong, L. P. A.; Benschop, H. P. Diagnosis and dosimetry of exposure to sulfur mustard: Development of a standard operating procedure for mass spectrometric analysis of haemoglobin adducts: Exploratory research on albumin and keratin adducts. Journal of Applied Toxicology 2000, 21, S187-S192.

87. van der Schans, G. P.; Noort, D.; Mars-Groenendijk, R. H.; Fidder, A.; Chau, L. F.; de Jong, L. P. A.; Benschop, H. P. Immunochemical detection of sulfur mustard adducts with keratins in the stratum corneum of human skin. Chemical Research in Toxicology 2002, 15 (1), 21-25.

88. Mol, M. A. E.; van den Berg, R. M.; Benschop, H. P. Proteomic assessment of sulfur mustard-induced protein adducts and other protein modifications in human epidermal keratinocytes. Toxicology and Applied Pharmacology 2008, 230 (1), 97-108.

89. Abel, E. L.; Bubel, J. D.; Simper, M. S.; Powell, L.; McClellan, S. A.; Andreef, M.; MacLeod, M. C.; DiGiovanni, J. Protection against 2-chloroethyl ethyl sulfide (CEES) - induced cytotoxicity in human keratinocytes by an inducer of the glutathione detoxification pathway. Toxicology and Applied Pharmacology 2011, $255,176-183$.

90. Price, E. O.; Smith, J. R.; Clark, C. R.; Schlager, J. J.; Shih, M. L. MALDI-TOF/MS as a Diagnostic Tool for the Confirmation of Sufur Mustard Exposure. Journal of Applied Toxicology 2000, 20, 193-197.

91. Smith, J. R.; Capacio, B. R.; Korte, W. D.; Woolfitt, A. R.; Barr, J. R. Analysis for plasma protein biomarkers following an accidental human exposure to sulfur mustard. Journal of Analytical Toxicology 2008, 32 (1), 17-24.

92. Tornqvist, M.; Mowrer, J.; Jensen, S.; Ehrenberg, L. Monitoring of Environmental Cancer Initiators through Hemoglobin Adducts by a Modified Edman Degradation Method. Analytical Biochemistry 1986, 154, 255-266.

93. Noort, D.; Hulst, A. G.; Jansen, R. Covalent binding of nitrogen mustards to the cysteine34 residue in human serum albumin. Archives of Toxicology 2002, 76 (2), 83-88.

94. Thulin, H.; Zorcec, V.; Segerback, D.; Sundwall, A.; Tornqvist, M. Oxazolidonylethyl adducts to hemoglobin and DNA following nornitrogen mustard exposure. Chemico-Biological Interactions 1996, 99 (1-3), 263-275.

95. Antoine, M.; Fabris, D.; Fenselau, C. Covalent sequestration of the nitrogen mustard mechlorethamine by metallothionein. Drug Metabolism and Disposition 1998, 26 (9), 921-926. 
96. Colvin, M. E.; Quong, J. N. DNA-Alkylating events associated with nitrogen mustard based anticancer drugs and the metabolic byproduct Acrolein. Advances in DNA Sequence-Specific Agents 2002, 4, 29-46.

97. Sarmah, N.; Neog, B.; Bhattacharyya, P. Kr. Affinity of aziridinium ion towards different nucleophiles: A density functional study. Computational and Theoretical Chemistry 2011, 976, 30-35.

98. Chait, B. T. Mass Spectrometry: Bottom-Up or Top-Down? Science 2006, 314, 65-66.

99. Hernandez, P.; Muller, M.; Appel, R. D. Automated protein identification by tandem mass spectrometry: Issues and strategies. Mass Spectrometry Reviews 2006, 25 (2), 235-254.

100. Simpson, R. J. Peptide Mapping and Sequence Analysis of Gel-Resolved Proteins. In Proteins and Proteomics: A Laboratory Manual, Cold Spring Harbor Laboratory Press: Cold Spring Harbor, NY, 2003; pp 343-424.

101. Ahmed, F. E. Utility of mass spectrometry for proteome analysis: part I. Conceptual and experimental approaches. Expert Review of Proteomics 2008, 5 (6), 841-864.

102. Konermann, L.; Ahadi, E.; Rodriguez, A. D.; Vahidi, S. Unraveling the Mechanism of Electrospray Ionization. Analytical Chemistry 2013, 85, 2-9.

103. Domon, B.; Aebersold, R. Review - Mass spectrometry and protein analysis. Science 2006, 312 (5771), 212-217.

104. de Hoffmann, E.; Stroobant, V. Mass Analyzers. In Mass Spectrometry: Principles and Applications, Third Edition ed.; John Wiley \& Sons: West Sussex, England, 2007; pp 85-173.

105. Rajski, S. R.; Williams, R. M. DNA Cross-Linking Agents and Antitumor Drugs. Chemical Reviews 1998, 98, 2723-2795.

106. Begleiter, A.; Mowat, M.; Israels, L. G.; Johnston, J. B. Chlorambucil in chronic lymphocytic leukemia: Mechanism of action. Leukemia \& Lymphoma 1996, 23 (3-4), 187-+.

107. Struck, R. F. Nitrogen Mustard and Related Structures. In Cancer Chemotherapeutic Agents, American Chemical Society: Washington, D.C., 1995; pp 112-121.

108. Colvin, O. M. An overview of cyclophosphamide development and clinical applications. Current Pharmaceutical Design 1999, 5 (8), 555-560.

109. Colvin, M. E.; Sasaki, J. C.; Tran, N. L. Chemical Factors in the Action of Phosphoramidic Mustard Alkylating Anticancer Drugs: Roles for Computational Chemistry. Current Pharmaceutical Design 1999, 5, 645-663.

110. Li, F.; Patterson, A. D.; Höffer, C. C.; Krausz, K. W.; Gonzalez, F. J.; Idle, J. R. Comparative metabolism of cyclophosphamide and ifosphamide in the mouse 
using UPLC-ESI-QTOFMS-based metabolomics. Biochemical Pharmacology 2010, 80, 1063-1074.

111. Helsby, N. A.; Goldthorpe, M. A.; Tang, M. H. Y.; Atwell, G. J.; Smith, E. M.; Wilson, W. R.; Tingle, M. D. Influence of mustard group structure on pathways of in vitro metabolism of anticancer N-(2-hydroxyethyl)-3,5-dinitrobenzamide 2mustard prodrugs. Drug Metabolism and Disposition 2008, 36 (2), 353-360.

112. Gu, Y.; Atwell, G. J.; Wilson, W. R. Metabolism and Excretion of the Novel Bioreductive Prodrug PR-104 in Mice, Rats, Dogs, and Humans. Drug Metabolism and Disposition 2010, 38 (3), 498-508.

113. Patterson, A. V.; Ferry, D. M.; Edmunds, S. J.; Gu, Y.; Singleton, R. S.; Patel, K.; Pullen, S. M.; Hicks, K. O.; Syddall, S. P.; Atwell, G. J.; Yang, S.; Denny, W. A.; Wilson, W. R. Mechanism of Action and Preclinical Antitumor Activity of the Novel Hypoxia-Activated DNA Cross-Linking Agent PR-104. Clinical Cander Research 2007, 13 (13), 3922-3932.

114. Hoes, I.; Van Dogen, F.; Lemiere, F.; Esmans, E. L.; Van Bockstaele, D.; Berneman, Z. N. Comparison between capillarty and nano liquid chromatography-electrospray mass spectrometry for the analysis of minor DNA-melphalan adducts. Journal of Chromatography B 2000, 748, 197-212.

115. Loeber, R.; Michaelson, E.; Fang, Q.; Campbell, C.; Pegg, A. E.; Tretyakova, N. CrossLinking of the DNA Repair Protein $\mathrm{O}^{6}$-Alkylguanine DNA Alkytransferase to DNA in the Presence of Antitumor Nitrogen Mustards. Chemical Research in Toxicology 2008, 21, 787-795.

116. Pettersson-Fernholm, T.; Vilpo, J.; Kosonen, M.; Hakala, K.; Hovinen, J. Reactions of 4bis(2-chloroethly)aminophenylacetic acid (phenylacetic acid mustard) in physiological solution. J. Chem. Soc. , Perkin Trans. 2 1999, (10), 2183-2187.

117. Metabolism of melphalan by rat liver microsomal glutathione S-transferase. ChemicoBiological Interactions 2005, 152, 101-106.

118. Dulik, D. M.; Fenselau, C.; Hilton, J. Characterization of mephalan-glutathione adducts whose formation is catalyzed by glutathione transferases. Biochemical Pharmacology 1986, 35 (19), 3405-3409.

119. Hubert, A. A.; Dirven, M.; van Ommen, B.; van Vladeren, P. J. Involvement of Human Glutathione S-Transferase Isoenzymes in the Conjugation of Cyclophosphamide Metabolites with Glutathione. Cancer Research 1994, 54, 6215-6220.

120. Prakash, C.; Sharma, R.; Gleave, M.; Nedderman, A. In Vitro Screening Techniques for Reactive Metabolites for Minimizing Bioactivation Potential in Drug Discovery. Current Drug Metabolism 2008, 9 (9), 952-964.

121. Eddershaw, P.; Dickins, M. Phase I Metabolism. In A Handbook of Bioanalysis and Drug Metabolism, Evans, G., Ed.; CRC Press: Boca Raton, 2004; pp 208-221. 
122. Manchee, G.; Dickins, M.; Pickup, E. Phase II Metabolism. In A Handbook of Bioanalysis and Drug Metabolism, Evans, G., Ed.; CRC Press: Boca Raton, 2004; pp 222-243.

123. Brandon, E. F. A.; Raap, C. D.; Meijerman, I.; Beijnen, J. H.; Schellens, J. H. M. An update on in vitro test methods in human hepatic drug biotransformation research: pros and cons. Toxicology and Applied Pharmacology 2003, 189, 233246.

124. Mitchell, M. D.; Elrick, M. M.; Walgren, J. L.; Mueller, R. A.; Morris, D. L.; Thompson, D. C. Peptide-based in vitro assay for the detection of reactive metabolites. Chemical Research in Toxicology 2008, 21 (4), 859-868.

125. Wisniewski, J. R.; Zougman, A.; Nagaraj, N.; Mann, M. Universal saple preparation method for proteome analysis. Nature Methods 2009, 6 (5), 359-363.

126. Protein Prospector. UCSF Mass Spectrometry Facility . http://prospector.ucsf.edu/prospecor/mshome/htm (Accessed December 17, 2013). Last updated 2013.

127. Bechtold, W. E.; Willis, J. K.; Sun, J. D.; Griffith, W. C.; Reddy, T. V. Biological Markers of Exposure to benzene: S-phenlycysteine in albumin. Carcinogenesis 1992, $13(7), 1217-1220$.

128. DeCaprio, A. P.; Kinney, E. A.; LoPachin, R. M. Comparative Covalent Protein Binding of 2,5-Hexanedione and 3-Acetyl-2,5-Hexanedione in the Rat. Journal of Toxicology and Environmental Health Part A 2009, 72, 861-869.

129. Schneider, K.; DeCaprio, A. P. Evaluation of in vitro metabolic systems for common drugs of abuse. 1. Cocaine. Xenobiotica 2013, 43 (12), 1043-1054.

130. Gaussian 03, Revision B.03, Frisch, M.J., Trucks, G.W., Schlegel, H.B., Scuseria, G.E., Robb, M.A. Cheeseman, J.R., Montgomery Jr., J.A., Vrven, T., Kudin, K.N., Burant, J.C., Millam, J.M., Iyengar, S.S., Tomasi, J., Barone, V., Mennucci, B., Cossi, M., Scalmani, G., Rega, N., Petersson, G.A., Nakatsuji, H., Hada, M., Ehara, M., Toyota, K., Fukuda, R., Hasegawa, J., Ishida, M., Nakajima, T., Honda, Y., Kitao, O., Nakai, H., Klene, M., Li, X., Knox, J.E., Hratchian, H.P., Cross, J.B., Bakken, V., Adamo, C., Jaramillo, J., Gomperts, R., Stratmann, R.E., Yazyev, O., Austin, A.J., Cammi, R., Pomelli, C.,Ochterski, J.W., Ayala, P.Y., Morokuma, K., Voth, G.A., Salvador, P., Dannenberg, J.J., Zakrewski, V.G., Dapprich, S., Daniels, A.D., Strain, M.C., Farkas, O., Malick, D.K., Rabuck, A.D., Raghavachari, K., Foresman, J.B., Ortiz, J.V., Cui, Q., Baboul, A.G., Clifford, S., Cioslowski, J., Stefanov, B.B., Liu, G., Liashenko, A., Piskorz,P., Komaromi, I., Martin, R.L., Fox, D.J., Keith, T., Al-Laham, M.A., Peng, C.Y., Nanayakkara, A., Challacombe, M., Gill, P.M.W., Johnson, B., Chen, W., Wong, M.W., Gonzalez, C., and Pople, J.A.; Gaussian, Inc., Wallingford CT, 2004

131. Rubino, F. M.; Pitton, M.; Di Fabio, D.; Colombi, A. Toward An "Omic" Physiopathology of Reactive Chemicals: Thirty Years of Mass Spectrometric 
Study of the Protein Adducts with Endogenous and Xenobiotic Compounds. Mass Spectrometry Reviews 2009, 28 (5), 725-784.

132. Lemire, S. W.; Ashley, D. L.; Calafat, A. M. Quantitative determination of the hydrolysis products of nitrogen mustards in human urine by liquid chromatographyelectrospray ionization tandem mass spectrometry. Journal of Analytical Toxicology 2003, 27 (1), 1-6.

133. Yeo, T. H.; Ho, M. L.; Loke, W. K. Development of a liquid chromatography-multiple reaction monitoring procedure for concurrent verification of exposure to different forms of mustard agents. Journal of Analytical Toxicology 2008, 32 (1), 51-56.

134. Tang, X. J.; Thibault, P.; Boyd, R. K. Fragmentation Reactions of Multiply-Protonated Peptides and Implications for Sequencing by Tandem Mass Spectrometry with Low-Energy Collision-Induced Dissociation. Analytical Chemistry 1993, 65, 2824-2834.

135. Koningsberg, W.; Guidotti, G.; Hill, R. J. The Amino Acid Sequence of the alpha chain of Human Hemoglobin. Journal of Biological Chemistry 1961, 236 (8), 55-56.

136. Pauling, L. Biochemistry. In General Chemistry, 3rd ed.; Dover Publications: NY, NY, 1988.

137. Jan, Y. H.; Heck, D. E.; Malaviya, R.; Casillas, R. P.; Laskin, D. L.; Laskin, J. D. CrossLinking of Thioredoxin Reductase by the Sulfur Mustard Analogue Mechlorethamine (Methylbis(2-chloroethyl)amine) in Human Lung Epithelial Cells and Rat Lung: Selective Inhibition of disulfide Reduction but Not Redox Cycling. Chemical Research in Toxicology 2013.

138. Steel, L. F.; Trotter, M. G.; Nakajima, P. B.; Mattu, T. S.; Gonye, G.; Block, T. Efficient and Specific Removal of Albumin from Human Serum Samples. Molecular \& Cellular Proteomics 2003, 2 (4), 262-270.

139. Faustman, E. M.; Omenn, G. S. Risk Assessment. In Casarett \& Doull's Toxicology: The Basic Science of Poisons, 7th Ed. ed.; Klaasen, C. D., Ed.; McGraw-Hill: New York, 2008; pp 107-128.

140. Hadidi, A. H. F. A.; Coulter, C. E. A.; Idle, J. R. Phenotypically Deficient Urinary Elimination of Carboxyphosphamide after Cyclophosphamide Administration to Cancer Patients. Cancer Research 1988, 48, 5167-5171.

141. Struck, R. F.; Kirk, M. C.; Mellett, L. B.; Darber, S. E.; Hill, D. L. Urinary metabolites of the Antitumor Agent Cyclophosphamide. Molecular Phoarmacology 1971, 7, 519-529.

142. Yule, S. M.; Boddy, A. V.; Cole, M.; Price, L.; Wyllie, R.; Tasso, M. J.; Pearson, A. D. J.; Idle, J. R. Cyclophosphamide Metabolism in Children. Cancer Research 1995, $55,803-809$. 
143. Alberts, D. S.; Chang, S. Y.; Chen, H. S. G.; Larcom, B. J.; Jones, S. E. Pharmacokinetics and metabolism of chlorambucil in man: a preliminary report. Cancer Treatment Reviews 1979, 6, 9-17.

144. McLean, A.; Woods, R. L.; Catovsky, D.; Farmer, P. Pharmacokinetics and metabolism of chlorambucil in patients with malignant disease. Cancer Treatment Reviews 1979, 6, 33-42.

145. McLean, A.; Newell, D.; Baker, G.; Connors, T. The Metabolism of Chlorambucil. Biochemical Pharmacology 1980, 29, 2039-2047.

146. Cullis, P. M.; Green, R. E.; Malone, M. E. Mechanism and reactivity of chlorambucil and chloramcubil-spermidine conjugate. Journal of the Chemical Society Perkin Transactions II 1995, 2 (7), 1503-1511.

147. Gunnarsson, P. O.; Johansson, S. Å.; Svensson, L. Cholesterol ester formation by transesterification of chlorambucil: a novel pathway in drug metabolism. Xenobiotica 1984, 14 (7), 569-574.

148. Hartley-Asp, B.; Gunnarsson, P. O.; Liljekvist, J. Cytotoxicity and metabolism of prednimustine, chlorambucil and prednisolone in a Chinese hamster cell line. Cancer Chemotherapy and Pharmacology 1986, 16, 85-90.

149. Lee, F. Y. F.; Coe, P.; Workman, P. Pharmacokinetic basis for the comparative antitumor activity and toxicity of chlorambucil, phenylacetic acid mustard, and $\beta, \beta$ difluorochlorambucil (CB 7103) in mice. Cancer Chemotherapy and Pharmacology 1986, 17, 21-29.

150. Stout, S. A.; Riley, C. M. The hydrolysis of L-phenylalanine mustard (melphalan). Internation Journal of Pharmaceutics 1985, 24, 193-208.

151. Zhang, J.; Ye, Z.; Lou, Y. Metabolism of melphalan by rat liver microsomal glutathione S-transferase. Chemico-Biological Interactions 2005, 152, 101-106.

152. Dulik, D. M.; Fenselau, C. Conversion of Melphalan to 4-(Glutathionyl)phenylalanine: A Novel Mechanism for Conjugation by Glutathionse-S-transferase. Drug Metabolism and Disposition 1987, 15 (2), 195-199.

153. Dugaiczyk, A.; Law, S. W.; Dennison, O. E. Nucleotide sequence and the encoded amino acids of human serum albumin mRNA. Proc. Natl. Acad. Sci. USA 1982, 79, 7175 .

154. Yang, I. S.; Kim, T. G.; Park, B. S.; Cho, K. J.; Lee, J. H.; Park, Y.; Kim, K. H. Crystal structures of aprotinin and its complex with sucrose octasulfate reveal multiple modes of interactions with implications for heparin binding. Biochemical and Biophysical Research Communications 2010, 397, 429-435. 


\section{APPENDICES}

Appendix 1: Biotransformation of Cyclophosphamide

Compiled based on (a) Colvin, O.M., ${ }^{108}$ (b) Hadidi et al., ${ }^{140}$ (c) Struck et al., ${ }^{141}$ and (d) Yule et al. ${ }^{142}$

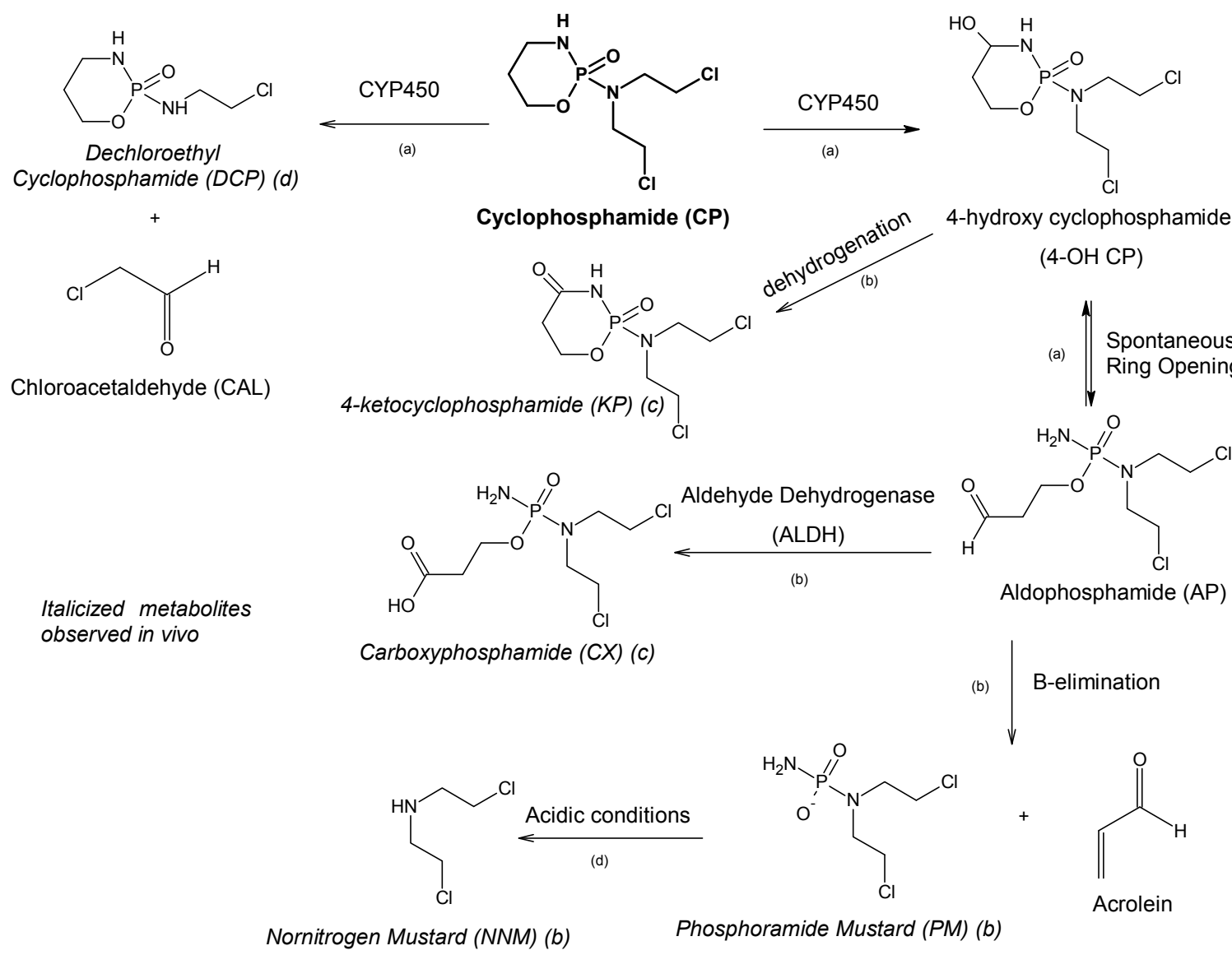


Appendix 2: Biotransformation of Chlorambucil

Compiled based on (a)Alberts et al., ${ }^{143}$ (b) McLean et al., ${ }^{144}$ (c) McLean et al., ${ }^{145}$ (d) Cullis et al., ${ }^{146}$ (e) Gunnarsson et al., ${ }^{147}$ (f) Hartley-Asp et al., ${ }^{148}$ (g) Lee et al., ${ }^{149}$ and (h)PetterssonFernholm et al. ${ }^{116}$

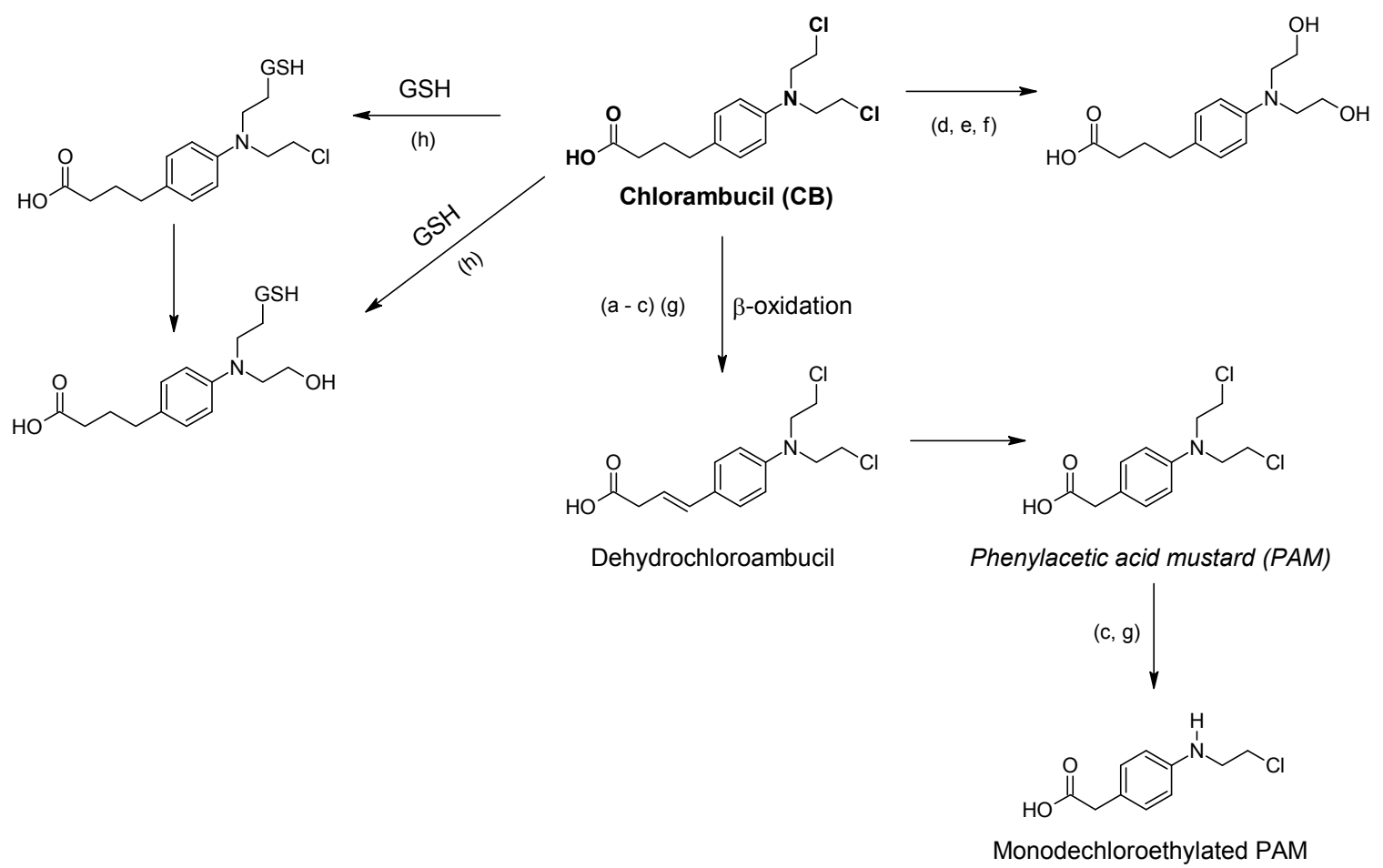


Appendix 3: Biotransformation of Melphalan

Compiled from (a) Stout and Riley, ${ }^{150}$ (b) Zhang et al., ${ }^{151}$ (c) Alaoui-Jamali et al., ${ }^{46}$ and (d) Dulik et al. ${ }^{152}$

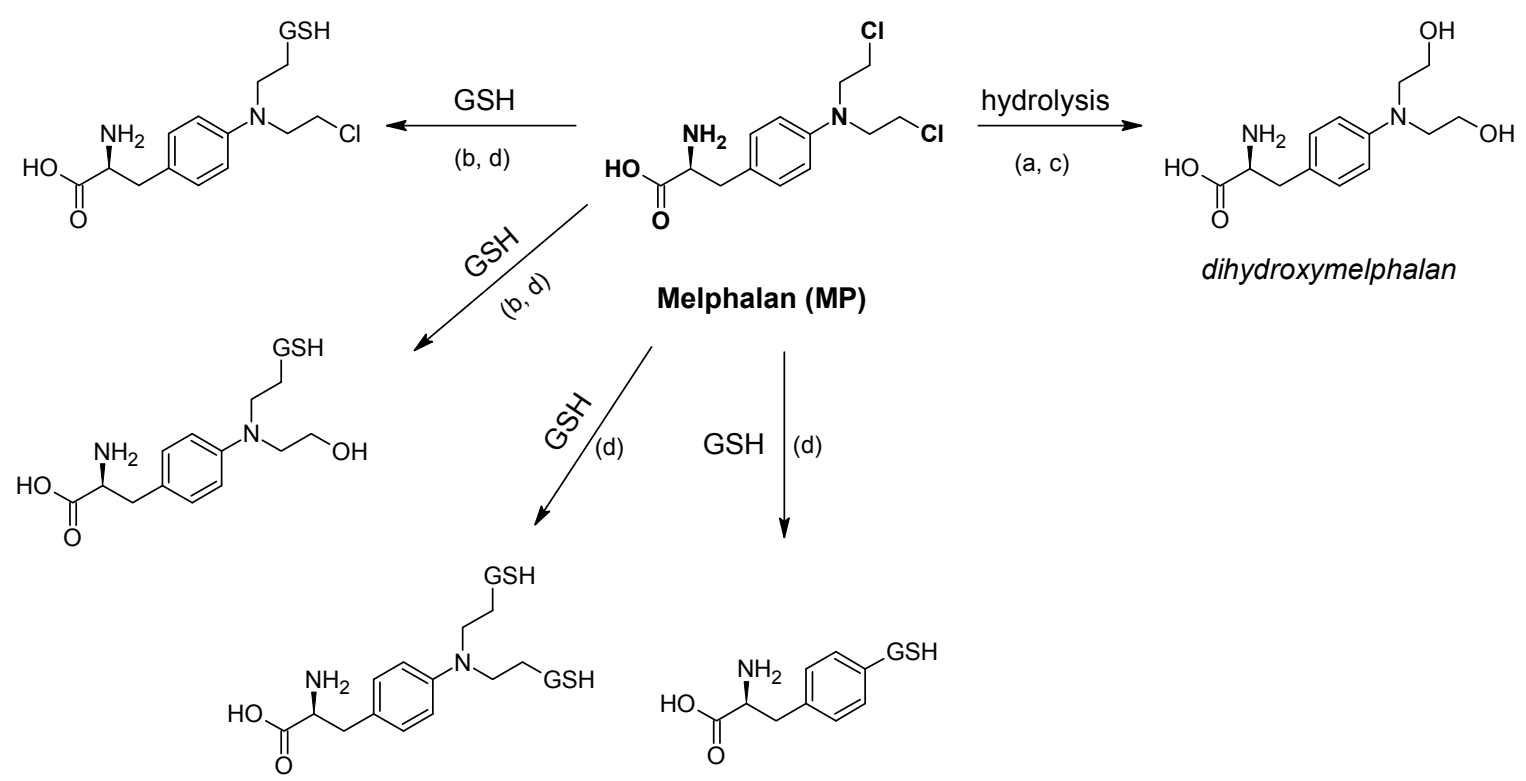


Appendix 4: MS/MS fragmentation of Model Peptides (a) AcPAACAA, (b) AcPAAKAA, and (c) AcPAAHAA
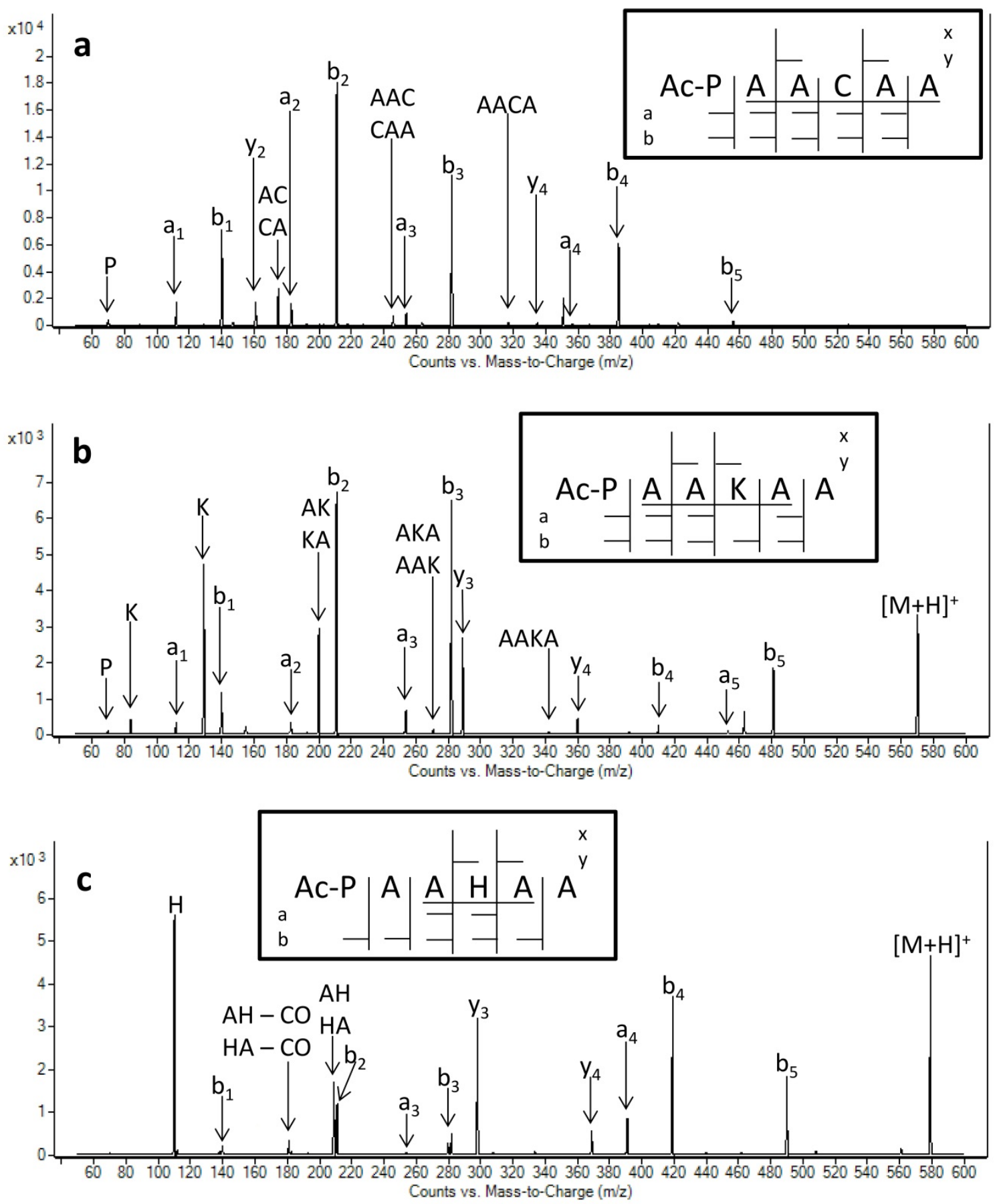
Appendix 5: BioConfirm Data Analysis Parameters for (a) Protein Deconvolution and (b) Protein Digest Analysis

a. Protein Deconvolution Parameters: Deconvolution (MS): Maximum Entropy

\begin{tabular}{|c|c|c|}
\hline \multirow{2}{*}{ Parameter } & \multicolumn{2}{|c|}{ Value } \\
\cline { 2 - 3 } & Hemoglobin & $\begin{array}{c}\text { Human Serum } \\
\text { Albumin }\end{array}$ \\
\hline Mass Range (Da) & $10,000-17,000$ & $50,000-70,000$ \\
\hline Mass Step & \multicolumn{2}{|c|}{$1 \mathrm{Da}$} \\
\hline Signal-to-Noise Threshold & \multicolumn{2}{|c|}{ Proton } \\
\hline Adduct & \multicolumn{2}{|c|}{5} \\
\hline Average Mass & $90 \%$ Peak Height & $75 \%$ Peak Height \\
\hline Minimum Consecutive Charge States & \multicolumn{2}{|c|}{8} \\
\hline Minimum Protein Fit Score & \multicolumn{2}{|c|}{} \\
\hline
\end{tabular}

b. Protein Digest Analysis:

\begin{tabular}{|c|c|}
\hline \multicolumn{2}{|c|}{ Find Compounds by Molecular Feature } \\
\hline Parameter & Value \\
\hline m/z Range & $350-3200 \mathrm{Da}$ \\
\hline Positive Ions allowed & $+\mathrm{H},+\mathrm{Na},+\mathrm{K},+\mathrm{NH}_{4}$ \\
\hline Yllow for Salt-dominated Positive Ions? & Small molecules (Chromatographic) \\
\hline Target Data Type & Use peaks with height $\geq 300$ counts \\
\hline Peak Filters & Peptides \\
\hline Isotope Model & Maximum charge state of 4 \\
\hline Charge State Limits & 0.0025 m/z, plus 7.0 ppm \\
\hline Isotope Grouping & Limit max number of peaks (by height) to largest 50 \\
\hline Peak Filters (MS/MS) & Extract Avg. MS/MS Spectrum for all CE \\
Precursor tolerance: \pm 20.00 ppm \\
Desults Reporting
\end{tabular}




\begin{tabular}{|c|c|}
\hline \multicolumn{2}{|r|}{ Define and Match Sequences: Hemoglobin } \\
\hline Sequences ${ }^{135,136}$ & $\begin{array}{l}\text { Alpha Subunit: } \\
\text { VLSPADKTNVKAAWGKVGAHAGEYGAEALERMFLSFPTKTYFPHFD } \\
\text { LSHGSAQVKGHGKKVADALTNAVAHVDDMPNALSALSDLHAHKLR } \\
\text { VDPVNFKLLSHCLLVTLAAHLPAEFTPAVHASLDKFLASVSTVLTSK } \\
\text { YR } \\
\text { Beta Subunit: } \\
\text { VHLTPEEKSAVTALWGKVNVDEVGGEALGRLLVVYPWTQRFFESRG } \\
\text { DLSTPDAVMGNPKVKAHGKKVLGAFSDGLAHLDNLKGTFATLSELH } \\
\text { CDKLHVDPENFRLLGNVLVCVLAHHFGKEFTPPVQAAYQKVVAGVA } \\
\text { NALAHKYH }\end{array}$ \\
\hline $\begin{array}{c}\text { Predicted } \\
\text { Modification }\end{array}$ & $\begin{array}{c}\text { Alkylation (Iodoacetamide): }+57.021464 \\
\text { Carbamylation: }+43.005184 \\
\text { Oxidation: }+15.994915 \\
\text { Deamidation: }+0.984016 \\
\text { HN-2 OH: }+101.084064 \\
\text { HN-2 Cl: }+119.050177 \\
\text { HN-3 OH: } 131.094629 \\
\text { HN-3 OH: } 149.060742\end{array}$ \\
\hline Mass Matching & $\begin{array}{l}\text { MS mass tolerance: } \pm 10.00 \mathrm{ppm} \\
\text { MS/MS mass tolerance: } \pm 50.00 \mathrm{ppm}\end{array}$ \\
\hline Scoring & $\begin{array}{c}\text { MS Score: } 30 \\
\text { MS/MS Score: } 70 \\
(50 \% \mathrm{MS} / \mathrm{MS} \text { peak intensity, } 50 \% \mathrm{MS} / \mathrm{MS} \text { matched ion score })\end{array}$ \\
\hline
\end{tabular}




\begin{tabular}{|c|c|}
\hline \multicolumn{2}{|r|}{ Define and Match Sequences: Human Serum Albumin } \\
\hline Sequence ${ }^{153}$ & $\begin{array}{l}\text { DAHKSEVAHRFKDLGEENFKALVLIAFAQYLQQCPFEDHVKLVNEVT } \\
\text { EFAKTCVAESAENCDKSLHTLFGDKLCTVATLRETYGEMADCCAKQ } \\
\text { EPERNECFLQHKDDNPNLPRLVRPEVDVMCTAFHDNEETFLKKYLYE } \\
\text { IARRHPYFYAPELLFFAKRYKAAFTECCQAADKAACLLPKLDELRDE } \\
\text { GKASSAKQRLKCASLQKFGERAFKAWAVARLSQRFPKAEFAEVSKL } \\
\text { VTDLTKVHTECCHGDLLECADDRADLAKYICENQDSISSKLKECCEKP } \\
\text { LLEKSHCIAEVENDEMPADLPSLAADFVESKDVCKNYAEAKDVFLGM } \\
\text { FLYEYARRHPDYSVVLLLRLAKTYETTLEKCCAAADPHECYAKVFDE } \\
\text { FKPLVEEPQNLIKQNCELFEQLGEYKFQNALLVRYTKKVPQVSTPTLV } \\
\text { EVSRNLGKVGSKCCKHPEAKRMPCAEDYLSVVLNQLCVLHEKTPVS } \\
\text { DRVTKCCTESLVNRRPCFSALEVDETYVPKEFNAETFTFHADICTLSE } \\
\text { KERQIKKQTALVELVKHKPKATKEQLKAVMDDFAAFVEKCCKADDK } \\
\text { ETCFAEEGKKLVAASQAALGL }\end{array}$ \\
\hline $\begin{array}{c}\text { Predicted } \\
\text { Modification }\end{array}$ & $\begin{array}{c}\text { Alkylation (Iodoacetamide): }+57.021464 \\
\text { Carbamylation: }+43.005184 \\
\text { Oxidation: }+15.994915 \\
\text { Deamidation: }+0.984016 \\
\text { HN-2 OH: }+101.084064 \\
\text { HN-2 Cl: }+119.050177 \\
\text { HN-3 OH: } 131.094629 \\
\text { HN-3 OH: } 149.060742\end{array}$ \\
\hline Mass Matching & $\begin{array}{l}\text { MS mass tolerance: } \pm 10.00 \mathrm{ppm} \\
\text { MS/MS mass tolerance: } \pm 50.00 \mathrm{ppm}\end{array}$ \\
\hline Scoring & $\begin{array}{c}\text { MS Score: } 30 \\
\text { MS/MS Score: } 70 \\
\text { (50\% MS/MS peak intensity, } 50 \% \mathrm{MS} / \mathrm{MS} \text { matched ion score) }\end{array}$ \\
\hline
\end{tabular}


Appendix 6: LC-MS and LC-MS/MS Parameters

1. Model Peptide Adduction and Metabolism Studies

a. Full Scan (Peptide and Metabolism Studies)

\begin{tabular}{|c|l|}
\hline Parameter & \multicolumn{1}{|c|}{ Value } \\
\hline & $0.0-0.5$ min: Hold at 10\% B \\
& $0.5-5.0$ min: Ramp from 10\% B to $25 \% \mathrm{~B}$ \\
Pump Program & $5.0-5.5$ min: Ramp from 25\% B to $95 \% \mathrm{~B}$ \\
& $5.5-7.0$ min: Hold at 95\% B \\
\hline Post-Time Equilibration & 7 minutes at 10\% B \\
\hline \multirow{2}{*}{ Solvent Composition } & A: $0.1 \%$ Trifluoroacetic acid (TFA) in $\mathrm{HPLC}_{2} \mathrm{O}$ \\
& B: $95: 5: 0.1 \%$ Acetonitrile: $\mathrm{H}_{2} \mathrm{O}: \mathrm{TFA}$ \\
\hline Flow Rate & $0.5 \mathrm{~mL} / \mathrm{min}$ \\
\hline Injection Volume & $2.0 \mu \mathrm{L}$ \\
\hline Column Temperature & $40 .{ }^{\circ} \mathrm{C}$ \\
\hline MS Mode & $\mathrm{MS} 2 \mathrm{Scan}(\mathrm{QQQ})$ \\
\hline MS Parameters & Frag: $140 \mathrm{~V}$ \\
\hline
\end{tabular}

b. Product Ion

\begin{tabular}{|c|c|}
\hline Parameter & Value \\
\hline Pump Program & $\begin{array}{l}0.0-0.5 \text { min: Hold at } 10 \% \mathrm{~B} \\
0.5-5.0 \text { min: Ramp from } 10 \% \mathrm{~B} \text { to } 25 \% \mathrm{~B} \\
5.0 \text { - } 5.5 \text { min: Ramp from } 25 \% \mathrm{~B} \text { to } 95 \% \mathrm{~B} \\
5.5-7.0 \text { min: Hold at } 95 \% \mathrm{~B}\end{array}$ \\
\hline Post-Time Equilibration & 7 minutes at $10 \% \mathrm{~B}$ \\
\hline Solvent Composition & $\begin{array}{l}\text { A: } 0.1 \% \text { Trifluoroacetic acid (TFA) in } \mathrm{HPLC}_{2} \mathrm{O} \\
\text { B: } 95: 5: 0.1 \% \text { Acetonitrile: } \mathrm{H}_{2} \mathrm{O}: \text { TFA }\end{array}$ \\
\hline Flow Rate & $0.5 \mathrm{~mL} / \mathrm{min}$ \\
\hline Injection Volume & $2.0 \mu \mathrm{L}$ \\
\hline Column Temperature & $40.0^{\circ} \mathrm{C}$ \\
\hline MS Mode & Product Ion (QQQ) \\
\hline MS Parameters & $\begin{array}{l}\text { Cys: Precursor Ions: 545, 646, 664, 676, } 694 \\
\text { Frag } 140 \mathrm{~V} \\
\text { CE: } 20 \mathrm{~V}, 25 \mathrm{~V}, 30 \mathrm{~V} \\
\text { Lys: Precursor Ions: } 570,671,689,701,719 \\
\text { Frag: } 140 \mathrm{~V} \\
\text { CE: } 20 \mathrm{~V}, 30 \mathrm{~V}, 40 \mathrm{~V} \\
\text { His: Precursor Ions: } 579,680,698,710,728 \\
\text { Frag: } 140 \mathrm{~V} \\
\text { CE: } 20 \mathrm{~V}, 30 \mathrm{~V}, 40 \mathrm{~V}\end{array}$ \\
\hline
\end{tabular}


2. Whole Protein Digest Studies

\begin{tabular}{|c|c|}
\hline \multicolumn{2}{|r|}{ Hemoglobin } \\
\hline Parameter & Value \\
\hline Pump Program & $\begin{array}{l}0.0-0.5 \text { min: Hold at } 10 \% \mathrm{~B} \\
0.5-15.0 \mathrm{~min}: \text { Ramp from } 10 \% \text { B to } 60 \% \mathrm{~B} \\
15.0-16.0 \mathrm{~min}: \text { Ramp from } 60 \% \text { B to } 100 \% \mathrm{~B} \\
16.0-21.0 \mathrm{~min}: \text { Hold at } 100 \% \mathrm{~B}\end{array}$ \\
\hline Post-Time Equilibration & 7 minutes at $10 \% \mathrm{~B}$ \\
\hline Solvent Composition & $\begin{array}{l}\text { A: } 0.1 \% \text { Trifluoroacetic acid (TFA) in } \mathrm{HPLC}_{2} \mathrm{O} \\
\text { B: } 95: 5: 0.1 \% \text { Acetonitrile: } \mathrm{H}_{2} \mathrm{O}: \text { TFA }\end{array}$ \\
\hline Flow Rate & $0.5 \mathrm{~mL} / \mathrm{min}$ \\
\hline Injection Volume & $3.0 \mu \mathrm{L}$ \\
\hline Column Temperature & $40.0^{\circ} \mathrm{C}$ \\
\hline MS Mode & Auto MS/MS (Q-TOF) \\
\hline MS Parameters & $\begin{array}{l}\text { Frag: } 175 \mathrm{~V} \\
\text { MS Mass range: } 100 \text { - } 3000 \mathrm{Da} \\
\text { MS Scan rate: } 4 \text { spectra/second } \\
\text { MS/MS Scan rate: } 3 \text { spectra/second } \\
\text { Isolation Width MS/MS: Medium }(\sim 4 \mathrm{amu}) \\
\text { Collision Energy Ramp: Slope } 3.7 \\
\text { Offset: } 2.5 \\
\text { Excluded Masses: } 121.050873 \mathrm{Da} \pm 100 \mathrm{ppm} \\
\text { 922.009798 Da } \pm 100 \mathrm{ppm} \\
\text { Precursor Selection: Max Precursors/cycle: } 3 \\
\text { Precursor threshold: }>1000 \text { cts. } \\
\text { Isotope Model: Peptides } \\
\text { Active exclusion after } 2 \text { spectra } \\
\text { Charge State Preference: }+2,+3,>+3\end{array}$ \\
\hline
\end{tabular}




\begin{tabular}{|c|c|}
\hline \multicolumn{2}{|r|}{ Human Serum Albumin } \\
\hline Parameter & Value \\
\hline Pump Program & $\begin{array}{l}0.0-0.5 \mathrm{~min}: \text { Hold at } 4 \% \mathrm{~B} \\
0.5-20.0 \mathrm{~min}: \text { Ramp from } 4 \% \text { B to } 48 \% \mathrm{~B} \\
20.0-21.0 \mathrm{~min}: \text { Ramp from } 48 \% \text { B to } 100 \% \text { B } \\
21.0-25.0 \mathrm{~min}: \text { Hold at } 100 \% \mathrm{~B}\end{array}$ \\
\hline Post-Time Equilibration & 7 minutes at $4 \% \mathrm{~B}$ \\
\hline Solvent Composition & $\begin{array}{l}\text { A: } 0.1 \% \text { Trifluoroacetic acid (TFA) in } \mathrm{HPLC}_{2} \mathrm{O} \\
\text { B: } 95: 5: 0.1 \% \text { Acetonitrile: } \mathrm{H}_{2} \mathrm{O}: \text { TFA }\end{array}$ \\
\hline Flow Rate & $0.5 \mathrm{~mL} / \mathrm{min}$ \\
\hline Injection Volume & $3.0 \mu \mathrm{L}$ \\
\hline Column Temperature & $40.0^{\circ} \mathrm{C}$ \\
\hline MS Mode & Auto MS/MS (Q-TOF) \\
\hline MS Parameters & $\begin{array}{l}\text { Frag: } 175 \text { V } \\
\text { MS Mass range: } 100 \text { - } 3000 \mathrm{Da} \\
\text { MS Scan rate: } 4 \text { spectra/second } \\
\text { MS/MS Scan rate: } 3 \text { spectra/second } \\
\text { Isolation Width MS/MS: Medium ( } 4 \mathrm{amu}) \\
\text { Collision Energy Ramp: Slope } 3.7 \\
\text { Offset: } 2.5 \\
\text { Excluded Masses: } 121.050873 \mathrm{Da} \pm 100 \mathrm{ppm} \\
\text { 922.009798 Da } \pm 100 \mathrm{ppm} \\
\text { Precursor Selection: Max Precursors/cycle: } 4 \\
\text { Precursor Threshold: }>1000 \text { cts. } \\
\text { Isotope Model: Peptides } \\
\text { Active exclusion after } 2 \text { spectra } \\
\text { Charge State Preference: }+2,+3,>+3\end{array}$ \\
\hline
\end{tabular}


3. $\mathrm{C}_{8}$ Whole Protein Analysis Studies

\begin{tabular}{|c|c|}
\hline Parameter & Value \\
\hline Pump Program & $\begin{array}{l}0.0-0.5 \text { min: Hold at } 10 \% \mathrm{~B} \\
0.5-20.0 \mathrm{~min}: \text { Ramp from } 10 \% \text { B to } 50 \% \mathrm{~B} \\
20.0-25.0 \mathrm{~min}: \text { Ramp from } 50 \% \text { B to } 60 \% \mathrm{~B} \\
25.0-26.0 \mathrm{~min}: \text { Ramp from } 60 \% \text { B to } 95 \% \mathrm{~B} \\
26.0-30.0 \text { min: Hold at } 95 \% \mathrm{~B}\end{array}$ \\
\hline Post-Time Equilibration & 7 minutes at $4 \%$ B \\
\hline Solvent Composition & $\begin{array}{l}\text { A: } 0.1 \% \text { Trifluoroacetic acid (TFA) in } \mathrm{HPLC} \mathrm{H}_{2} \mathrm{O} \\
\text { B: } 95: 5: 0.1 \% \text { Acetonitrile: } \mathrm{H}_{2} \mathrm{O}: \text { TFA }\end{array}$ \\
\hline Flow Rate & $0.5 \mathrm{~mL} / \mathrm{min}$ \\
\hline Injection Volume & $10.0 \mu \mathrm{L}$ \\
\hline Column Temperature & $40.0^{\circ} \mathrm{C}$ \\
\hline MS Mode & MS Mode (Q-TOF) \\
\hline MS Parameters & $\begin{array}{l}\text { Frag: } 175 \text { V } \\
\text { MS Mass range: } 100-3000 \mathrm{Da} \\
\text { MS Scan rate: } 4 \text { spectra/second } \\
\text { Collision Energy Ramp: Slope } 3.7 \\
\text { Offset: } 2.5 \\
\text { Excluded Masses: } 121.050873 \mathrm{Da} \pm 100 \mathrm{ppm} \\
\text { 922.009798 Da } \pm 100 \mathrm{ppm} \\
\text { Precursor Selection: Max Precursors/cycle: } 4 \\
\text { Precursor Threshold: }>1000 \text { cts. } \\
\text { Isotope Model: Peptides } \\
\text { Active exclusion after } 2 \text { spectra } \\
\text { Charge State Preference: }+2,+3,>+3\end{array}$ \\
\hline
\end{tabular}

4. SIM Metabolism Studies

\begin{tabular}{|c|l|}
\hline Parameter & \multicolumn{1}{|c|}{ Value } \\
\hline & $1.0-0.5$ min: Hold at $10 \% \mathrm{~B}$ \\
Pump Program & $0.5-5.0$ min: Ramp from $10 \%$ B to $25 \% \mathrm{~B}$ \\
& $5.0-5.5$ min: Ramp from $25 \%$ B to $95 \% \mathrm{~B}$ \\
& $5.5-7.0$ min: Hold at $95 \% \mathrm{~B}$ \\
\hline Post-Time Equilibration & 7 minutes at $10 \% \mathrm{~B}$ \\
\hline \multirow{2}{*}{ Solvent Composition } & A: $0.1 \%$ Trifluoroacetic acid (TFA) in HPLC $\mathrm{H}_{2} \mathrm{O}$ \\
& B: $95: 5: 0.1 \%$ Acetonitrile: $\mathrm{H}_{2} \mathrm{O}: \mathrm{TFA}$ \\
\hline Flow Rate & $0.5 \mathrm{~mL} / \mathrm{min}$ \\
\hline Injection Volume & $2.0 \mu \mathrm{L}$ \\
\hline Column Temperature & $40.0{ }^{\circ} \mathrm{C}$ \\
\hline MS Mode & Product Ion (QQQ) \\
\hline & Frag (all): $140 \mathrm{~V}$ \\
MS Parameters & Cys: SIM Ions: $646,664,676,694$ \\
& $\underline{\text { Lys: SIM Ions: } 671,689,701,719}$ \\
& $\underline{\text { His: SIM Ions: } 680,698,710,728}$ \\
\hline
\end{tabular}


Appendix 7: Protein Prospector Data Analysis Parameters

Available online at http://www.prospector/ucsf.edu/prospector/mshome/htm

Version of software utilized at completion of project: $\mathrm{v} 5.10 .15$

MS-Product Program utilized for labeling of tryptic peptide MS-MS fragments

\begin{tabular}{|c|c|}
\hline Parameter & Value \\
\hline Sequence & Based on hypothesized match done by BioConfirm Software. \\
& $\begin{array}{l}\text { HN modification represented by parenthesis followed by exact mass of } \\
\text { modification (as seen in Appendix 5) }\end{array}$ \\
\hline N-term Sequence & a, b \\
\hline C-term Sequence & x, y \\
\hline Internal Fragment & Allow for Internal Fragmentation \\
\hline Neutral-loss & $-\mathrm{H}_{2} \mathrm{O}(\mathrm{S}, \mathrm{T}, \mathrm{E}, \mathrm{D})$ \\
Sequence & $-\mathrm{NH}_{3}(\mathrm{R}, \mathrm{K}, \mathrm{Q}, \mathrm{N})$ \\
\hline Peeling Sequence & $\mathrm{b}+\mathrm{H}_{2} \mathrm{O}(\mathrm{R}, \mathrm{H}, \mathrm{K})$ \\
\hline Max Charge & 2 \\
\hline Instrument & ESI-Q-TOF \\
\hline
\end{tabular}


Appendix 8: Components of Protease Inhibitor Cocktail

Purchased from Sigma-Aldrich, Catalog Number: P8340
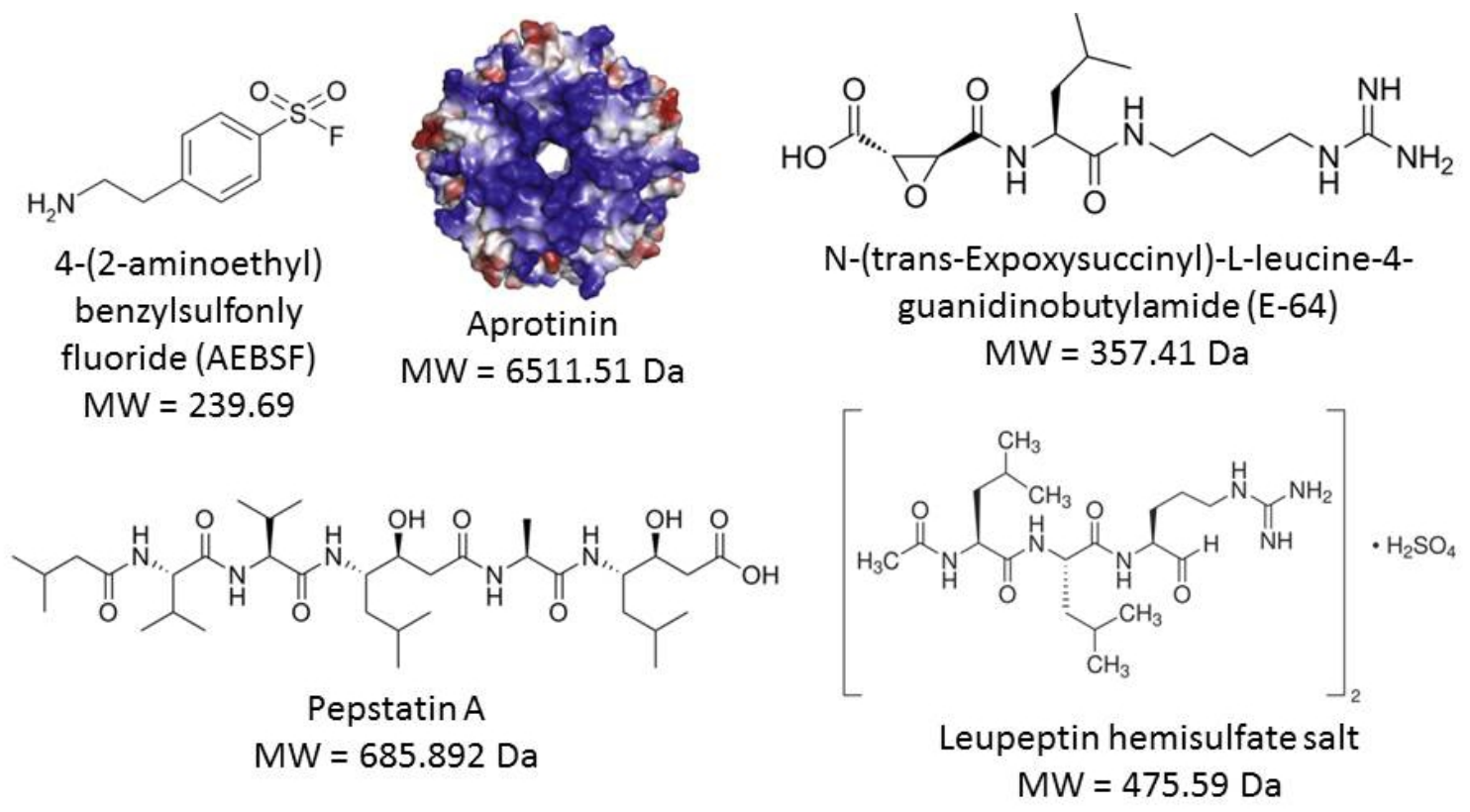

Figure for Aprotinin obtained from Yang et al. ${ }^{154}$

Components are removed prior to trypsin digestion via $10 \mathrm{kDa}$ spin filters. 
Appendix 9: Calculated Reactivity Index Data for HN-2 and HN-3 Electrophilic Intermediates

Note: As it is expected that the aziridinium species are those responsible for adduction, data was only calculated for these intermediates. Cys, Lys, and His data have been highlighted in red for comparison purposes.

\begin{tabular}{|c|c|}
\hline \multicolumn{2}{|c|}{ Electrophile: HN-2 Az OH } \\
\hline Amino Acid (Charge) & $\begin{array}{c}\text { Reactivity Index ( } \mathbf{(} \text { ) } \\
\text { (eV) }\end{array}$ \\
\hline Serine (-1) & 2.660377271 \\
\hline Threonine (-1) & 2.604330541 \\
\hline Cysteine (-1) & 2.46414707 \\
\hline Aspartic Acid (-1) & 2.137008716 \\
\hline Glutamic Acid (-1) & 1.910437929 \\
\hline Tryptophan (0) & 0.602879711 \\
\hline Tyrosine (0) & 0.594932818 \\
\hline Arginine (0) & 0.556125457 \\
\hline Lysine (0) & 0.541671492 \\
\hline Asparagine (0) & 0.529160347 \\
\hline Threonine (0) & 0.519029658 \\
\hline Glutamine (0) & 0.516742297 \\
\hline Histidine (0) & 0.515973467 \\
\hline Serine (0) & 0.513658011 \\
\hline Glutamic Acid (0) & 0.472297531 \\
\hline Aspartic Acid (0) & 0.45104694 \\
\hline Cysteine (0) & 0.424372333 \\
\hline Arginine (1) & 0.014942882 \\
\hline Lysine (1) & 0.008672125 \\
\hline Histidine (1) & 0.002468309 \\
\hline
\end{tabular}




\begin{tabular}{|c|c|}
\hline \multicolumn{2}{|c|}{ Electrophile: HN-3 Az OH OH } \\
\hline $\begin{array}{c}\text { Amino Acid } \\
\text { (Charge) }\end{array}$ & $\begin{array}{c}\text { Reactivity Index } \\
(\mathbf{(})^{\mathbf{C}} \mathbf{( e V )}\end{array}$ \\
\hline Serine (-1) & 2.743354059 \\
\hline Threonine (-1) & 2.684758899 \\
\hline Cysteine (-1) & 2.5417167 \\
\hline Aspartic Acid (-1) & 2.201480744 \\
\hline Glutamic Acid (-1) & 1.973955994 \\
\hline Tryptophan (0) & 0.615134425 \\
\hline Tyrosine (0) & 0.606129284 \\
\hline Arginine (0) & 0.565856589 \\
\hline Lysine (0) & 0.550613803 \\
\hline Asparagine (0) & 0.537226337 \\
\hline Threonine (0) & 0.527228875 \\
\hline Histidine (0) & 0.525503578 \\
\hline Glutamine (0) & 0.524699006 \\
\hline Serine (0) & 0.521675972 \\
\hline Glutamic Acid (0) & 0.479097983 \\
\hline Aspartic Acid (0) & 0.457599431 \\
\hline Cysteine (0) & 0.429982528 \\
\hline Arginine (1) & 0.014074194 \\
\hline Lysine (1) & 0.007937744 \\
\hline Histidine (1) & 0.003189918 \\
\hline
\end{tabular}

\begin{tabular}{|c|c|}
\hline \multicolumn{2}{|c|}{ Electrophile: HN-3 Az OH Cl } \\
\hline $\begin{array}{c}\text { Amino Acid } \\
\text { (Charge) }\end{array}$ & $\begin{array}{c}\text { Reactivity Index } \\
\left(\mathbf{\omega}^{-}\right)(\mathbf{e V})\end{array}$ \\
\hline Serine (-1) & 3.188096357 \\
\hline Threonine (-1) & 3.121836274 \\
\hline Cysteine (-1) & 2.964150359 \\
\hline Aspartic Acid (-1) & 2.580587256 \\
\hline Glutamic Acid (-1) & 2.330777185 \\
\hline Tryptophan (0) & 0.775460515 \\
\hline Tyrosine (0) & 0.764114282 \\
\hline Arginine (0) & 0.716775779 \\
\hline Lysine (0) & 0.698446552 \\
\hline Asparagine (0) & 0.681936331 \\
\hline Threonine (0) & 0.670957056 \\
\hline Histidine (0) & 0.67075383 \\
\hline Glutamine (0) & 0.667702212 \\
\hline Serine (0) & 0.664411073 \\
\hline Glutamic Acid (0) & 0.614377979 \\
\hline Aspartic Acid (0) & 0.589661246 \\
\hline Cysteine (0) & 0.556747799 \\
\hline Arginine (1) & 0.03664921 \\
\hline Lysine (1) & 0.025686349 \\
\hline Histidine (1) & 0.000108479 \\
\hline
\end{tabular}




\section{VANESSA THOMPSON}

$2004-2008$

B.S., Chemistry and Psychology, cum laude Vanderbilt University

Nashville, TN

$2009-2014$

Doctoral Candidate, Chemistry

Florida International University

Miami, FL

\section{PUBLICATIONS AND PRESENTATIONS}

Thompson, V.R. and DeCaprio, A.P. Protein Adduct Based Biomarkers of Chemical Warfare Agents: Characterization of HN-2 and HN-3 Adduction to Model Peptides. Presented at the joint meeting of the Society of Forensic Toxicologist and The International Association of Forensic Toxicologists (SOFT-TIAFT) Meeting; San Francisco, CA, USA, 2011.

Thompson, V.R. and DeCaprio, A.P. Protein Adduct Based Biomarkers of Chemical Warfare Agents: Characterization of HN-2 and HN-3 Adduction to Model Peptides. Presented at the International Forensics Research Institution Forensic Science Symposium; Miami, FL, USA 2012 .

Thompson, V.R. and DeCaprio, A.P. Protein Adduct Based Biomarkers of Chemical Warfare Agents: Characterization of HN-2 and HN-3 Adduction to Model Peptides. Presented at the Florida International University Graduate Student Scholarly Forum; Miami, FL, USA 2012. Awarded First Place Poster Presentation.

Thompson, V.R. and DeCaprio, A.P. Characterization of Protein Adduction of Nitrogen Mustard Chemical Warfare Agents to Model Peptides. Presented at the Florida Annual Meeting and Expo (FAME); Tampa, FL, USA, 2012.

Thompson, V.R. and Graves. P. General Chemistry I Laboratory Manual. Florida International University Department of Chemistry and Biochemistry, 2012.

Thompson, V.R. and Graves. P. General Chemistry II Laboratory Manual. Florida International University Department of Chemistry and Biochemistry, 2012.

Thompson, V.R. and DeCaprio, A.P. Characterization of Protein Adducts of Nitrogen Mustards to Peptides and Protein as Potential Exposure Biomarkers. Presented at the Society of Toxicology (SOT) Meeting; San Antonio, TX, USA, 2013. Awarded Graduate Student Travel Grant.

Thompson, V.R. and DeCaprio, A.P. Covalent Adduction of Nitrogen Mustards to Model Protein Nucleophilies. Chemical Research in Toxicology, 2013, 26, $1263-1271$. 
Thompson, V.R. and DeCaprio, A.P. Protein Adducts of Nitrogen Mustard Chemical Warfare Agents to Human Serum Albumin as Potential Biomarkers of Exposure. Presented at the Society of Forensic Toxicology (SOFT) Meeting; Orlando, FL, USA, 2013.

Thompson, V.R. and DeCaprio, A.P. Characterization of Protein Adducts of Nitrogen Mustards as Potential Exposure Biomarkers. Presented at the Florida International University Graduate Student Scholarly Forum; Miami, FL, USA, April 2013. Awarded Third Place Platform Presentation.

Thompson, V.R. and DeCaprio, A.P. Utilizing Protein Adducts as Exposure Biomarkers for Nitrogen Mustard Chemical Warfare Agents. Presented at the American Academy of Forensic Sciences (AAFS) Annual Meeting; Seattle, WA, USA, 2014.

Thompson, V.R. and DeCaprio, A.P. Chemical Warfare Agents: Past, Present, and Future of Toxicological Detection. Presented at the International Forensic Research Institute Forensic Science Symposium; Miami, FL, USA, March 2014.

Thompson, V.R. and DeCaprio, A.P. Characterization of Protein Adducts of Nitrogen Mustard Chemical Warfare Agents to Human Serum Albumin. Presented at the Florida International University Graduate Student Scholarly Forum; Miami, FL, USA, April 2014.

Thompson, V.R. and DeCaprio, A.P. Identification and Characterization of Covalent Adduction of Nitrogen Mustard Chemical Warfare Agents HN-2 and HN-3 to Human Hemoglobin as Potential Biomarkers of Exposure. (In Preparation)

Thompson, V.R. and DeCaprio, A.P. Identification and Characterization of Covalent Adduction of Nitrogen Mustard Chemical Warfare Agents HN-2 and HN-3 to Human Serum Albumin as Potential Biomarkers of Exposure. (In preparation). 